\title{
Contributions to solve the Multi-group Neutron Transport Equation with different Angular Approaches
}

\author{
PhD Thesis
}

Author:

Sergio Morató Rafet

Supervisors: Prof. Rafael Miró Herrero Dr. Álvaro Bernal García 



\section{Contributions to solve the Multi-group Neutron Transport Equation with different Angular Approaches}

September 2020

Author: $\quad$ Sergio Morató Rafet

Supervisors: Professor Rafael Miró Herrero

Dr. Álvaro Bernal García 

Fantasía X

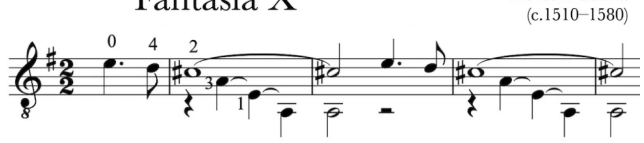





\section{Abstract}

The most accurate way to know the movement of the neutrons through matter is achieved by solving the Neutron Transport Equation. Three different approaches to solve this equation have been investigated in this thesis: Discrete Ordinates Neutron Transport Equation, Neutron Diffusion Equation and Simplified Spherical Harmonics Equations.

In order to solve the equations, different schemes of the Finite Differences Method were studied. The solution of these equations describes the population of neutrons and the occurred reactions inside a nuclear system. These variables are related with the flux and power, fundamental parameters for the Nuclear Safety Analysis.

The thesis introduces the definition of the mentioned equations. In particular, they are detailed for the steady state case. The Modal Method is proposed as a solution to the eigenvalue problems determined by the equations.

First, several algorithms for the solution of the steady state of the Neutron Transport Equation with the Discrete Ordinates Method for the angular discretization and Finite Difference Method for spatial discretization are developed. A formulation able to solve eigenvalue problems for any number of energy groups, with scattering and anisotropy has been developed. Several quadratures used by this method for the angular discretization have been studied and implemented for any order of approach of the discrete ordinates. Furthermore, an adapted formulation has been developed as a solution of the source problem for the Neutron Transport Equation. 
Next, an algorithm is carried out that allows to solve the Neutron Diffusion Equation with two variants of the Finite Difference Method, one with cell centered scheme and another edge centered. The Modal method is also used for calculating any number of eigenvalues for several energy groups and upscattering.

Both Finite Difference schemes mentioned before are also implemented to solve the Simplified Spherical Harmonics Equations. Moreover, an analysis of different approaches of the boundary conditions is performed.

Finally, calculations of the multiplication factor, subcritical modes, neutron flux and the power for different nuclear reactors were carried out. These variables result essential in Nuclear Safety Analysis. In addition, several sensitivity studies of parameters like mesh size, quadrature order or quadrature type were performed. 


\section{Resumen}

La forma más exacta de conocer el desplazamiento de los neutrones a través de un medio material se consigue resolviendo la Ecuación del Transporte Neutrónico. Tres diferentes aproximaciones de esta ecuación se han investigado en esta tesis: Ecuación del transporte neutrónico resuelta por el método de Ordenadas Discretas, Ecuación de la Difusión y Ecuación de Armónicos Esféricos Simplificados.

Para resolver estás ecuaciones se estudian diferentes esquemas del Método de Diferencias Finitas. La solución a estas ecuaciones describe la población de neutrones y las reacciones ocasionadas dentro de un reactor nuclear. A su vez, estas variables están relacionadas con el flujo y la potencia, parámetros fundamentales para el Análisis de Seguridad Nuclear.

La tesis introduce la definición de las ecuaciones mencionadas y en particular se detallan para el estado estacionario. Se plantea el Método Modal como solución a los problemas de autovalores definidos por dichas ecuaciones.

Primero se desarrollan varios algoritmos para la resolución del estado estacionario de la Ecuación del Transporte de Neutrones con el Método de Ordenadas Discretas para la discretización angular y el Método de Diferencias Finitas para la discretización espacial. Se ha implementado una formulación capaz de resolver el problema de autovalores para cualquier número de grupos energéticos con upscattering y anisotropía. Varias cuadraturas utilizadas por este método en su resolución angular han sido estudiadas e implementadas para cualquier orden de aproximación de Ordenadas Discretas. Además, otra formulación se desarrolla para la solución del problema fuente de la ecuación del transporte neutrónico. 
A continuación, se lleva a cabo un algoritmo que permite resolver la Ecuación de la Difusión de Neutrones con dos variantes del método de diferencias Finitas, una centrada en celda y otra en vértice o nodo. Se utiliza también el Método Modal calculando cualquier número de autovalores para varios grupos de energía y con upscattering.

También se implementan los dos esquemas del Método de Diferencias Finitas anteriormente mencionados en el desarrollo de diferentes algoritmos para resolver las Ecuaciones de Armónicos Esféricos Simplificados. Además, se ha realizado un análisis de diferentes aproximaciones de las condiciones de contorno.

Finalmente, se han realizado cálculos de la constante de multiplicación, los modos subcríticos, el flujo neutrónico y la potencia para diferentes tipos de reactores nucleares. Estas variables resultan esenciales en Análisis de Seguridad Nuclear. Además, se han realizado diferentes estudios de sensibilidad de parámetros como tamaño de malla, orden utilizado en cuadraturas o tipo de cuadraturas. 


\section{Resum}

La forma més exacta de conéixer el desplaçament dels neutrons a través d'un mitjà material s'aconsegueix resolent l'Equació del Transport Neutrònic. Tres diferents aproximacions d'esta equació s'han investigat en aquesta tesi: Equació del Transport Neutrònic resolta pel mètode d'Ordenades Discretes, Equació de la Difusió i Equació d’Ármonics Esfèrics Simplificats.

Per a resoldre estes equacions s'estudien diferents esquemes del Mètode de Diferències Finites. La solució a estes equacions descriu la població de neutrons i les reaccions ocasionades dins d'un reactor nuclear. Al seu torn, estes variables estan relacionades amb el flux i la potència, paràmetres fonamentals per a l'Anàlisi de Seguretat Nuclear. La tesi introduix la definició de les equacions mencionades i en particular es detallen per a l'estat estacionari. Es planteja el Mètode Modal com a solució als problemes d'autovalors definits per les dites equacions.

Primer es desenvolupen diversos algoritmes per a la resolució de l'estat estacionari de l'Equació del Transport de Neutrons amb el Mètode d'Ordenades Discretes per a la discretització angular i el Mètode de Diferències Finites per a la discretització espacial. S'ha implementat una formulació capaç de resoldre el problema d'autovalors per a qualsevol nombre de grups energètics amb upscattering $\mathrm{i}$ anisotropia. Diverses quadratures utilitzades per este mètode en la seua resolució angular han sigut estudiades i implementades per a qualsevol orde d'aproximació d'Ordenades Discretes. A més, una altra formulació es desenvolupa per a la solució del problema font de l'Equació del Transport Neutrònic.

A continuació, es du a terme un algoritme que permet resoldre l'Equació de la Difusió de Neutrons amb dos variants del mètode de Diferències Finites, una cen- 
trada en cel-la i una altra en vèrtex o node. S'utilitza també el Mètode Modal calculant qualsevol nombre d'autovalors per a diversos grups d'energia i amb upscattering. També s'implementen els dos esquemes del Mètode de Diferències Finites aneriormente mencionats en el desenvolupament de diferents algoritmes per a resoldre les Equacions d'Harmònics Esfèrics Simplificats. A més, s'ha realitzat una anàlisi de diferents aproximacions de les condicions de contorn.

Finalment, s'han realitzat càlculs de la constant de multiplicació, els modes subcrítics, el flux neutrònic i la potència per a diferents tipus de reactors nuclears. Estes variables resulten essencials en Anàlisi de Seguretat Nuclear. A més, s'han realitzat diferents estudis de sensibilitat de paràmetres com la grandària de malla, orde utilitzat en quadraturas o tipus de quadratures. 


\section{Acknowledgments}

First, I would like to thank my thesis supervisors Rafael Miró and Álvaro Bernal. I would like to thank Rafael Miró for giving me the opportunity to develop professionally in the field of the nuclear technology and for allowing me to do it in both areas, in the field of medical applications and radiation protection and in the field of neutronics and code programming. I would like to thank Álvaro Bernal, for guiding my steps and for being an example for me to follow.

Secondly, I would also like to thank the Ministry of Economy, Industry and Competitiveness and the State Research Agency of Spain for the granting of my predoctoral contract for Research Personnel Training with reference BES-2016-076782. The financial aid provided by this contract was essential for the development of this thesis, as well as for the financing of a stay.

I would also like to especially thank Thomas Downar, Edward Larsen, and Brendan Kochunas for the time they gave me during my stay at the University of Michigan, Ann Arbor, USA. A special mention to Edward Larsen for whom I already felt a great admiration and even more after meeting him in person, for his talks and advice. Not also forgetting Andrew Ward and Volkan Seker for making me feel at home and in general all the colleagues with whom I shared moments in the Nuclear Engineering and Radiological Sciences Department (NERS).

On the other hand, I also want to thank all those who make up the Department of Chemical and Nuclear Engineering of the Polytechnic University of Valencia. In particular, I would like to thank Gumersindo Verdú for his continuous research discussions; Belén Juste for his great help, advice and collaboration in many investigations; José Rodenas for always offering me new training and Antoni Vidal 
for sharing his knowledge. In the same way, I would like to extend my thanks to my department colleagues, especially Carles, Consuelo and Antonella, but without forgetting Amanda, Patricio, Aina, Nico, María, Javier, Sete and Marina.

I thank my friends from Valencia and Denia for all their details and for always being present in my day to day.

Last but not least, I appreciate the support of my family, my brothers and my father; and those who make me feel like family, Jesús and Nati. I especially want to thank María, for always being by my side and inviting me to improve myself. Finally, it would be unfair not to remember my mother to whom I owe everything that I am. 


\section{Agradecimientos}

En primer lugar, quiero dar las gracias a mis directores de tesis Rafael Miró y Álvaro Bernal. Quiero agradecer a Rafael Miró por haberme brindado la oportunidad de desarrollarme profesionalmente en el campo de la tecnología nuclear y permitirme hacerlo tanto, en el ámbito de las aplicaciones médicas y protección radiológica como en el de la neutrónica y programación de códigos. Quiero agradecer a Álvaro Bernal, por guiar mis pasos y ser para mí un ejemplo a seguir.

En segundo lugar, me gustaría dar las gracias también al Ministerio de Economía, Industria y Competitividad y a la Agencia Estatal de Investigación de España por la concesión de mi contrato predoctoral de Formación de Personal Investigador con referencia BES-2016-076782. La ayuda económica proporcionada por este contrato fue esencial para el desarrollo de esta tesis, así como para el financiamiento de una estancia.

También me gustaría agradecer especialmente a Thomas Downar, Edward Larsen y Brendan Kochunas por el tiempo que me brindaron durante mi estancia en University of Michigan, Ann Arbor, USA. Una mención especial a Edward Larsen por quien ya sentía una gran admiración y más aún después de conocerlo en persona, por sus charlas y consejos. Sin olvidar también a Andrew Ward y Volkan Seker por hacerme sentir como en casa y en general a todos los compañeros con los que compartí momentos en el Nuclear Engineering and Radiological Sciences Department (NERS).

Por otro lado, también quiero dar las gracias a todos los que integran el Departamento de Ingeniería Química y Nuclear de la Universidad Politécnica de Valencia. En particular agradecer a Gumersindo Verdú, sus continuos debates en 
investigación; a Belén Juste por su gran ayuda, asesoramiento y colaboración en muchas investigaciones; a José Rodenas por ofrecerme siempre una nueva formación y a Antoni Vidal por compartir sus conocimientos. De la misma forma, quisiera extender mis agradecimientos a mis compañeros de departamento, en especial a Carles, Consuelo y Antonella, pero sin olvidarme de Amanda, Patricio, Aina, Nico, María, Javier, Sete y Marina.

Agradezco a mis amigos de Valencia y Denia todos sus detalles y por estar siempre presentes en mi día a día.

Por último, pero no menos importante, agradezco el apoyo de mi familia, mis hermanos y mi padre; y a los que me hacen sentir como tal, Jesús y Nati. Muy especialmente quiero dar las gracias a María, por estar siempre a mi lado e invitar a superarme. Finalmente, sería injusto no acordarme de mi madre a quien debo todo lo que soy. 


\section{Contents}

Abstract $\quad$ V

Acknowledgments $\quad$ xi

$\begin{array}{ll}\text { Contents } & \text { XV }\end{array}$

List of Figures $\quad$ xix

List of Tables $\quad$ xxvii

List of Symbols $\quad$ xxxi

1 Introduction 1

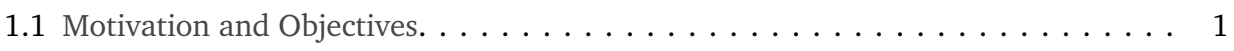

1.2 Thesis outline. . . . . . . . . . . . . . . . 3

2 State of the art $\quad 5$

2.1 The Transport Equation $\ldots \ldots \ldots \ldots \ldots \ldots \ldots \ldots \ldots$

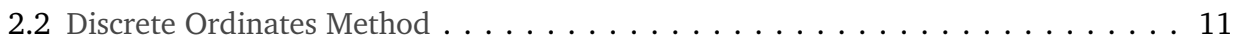




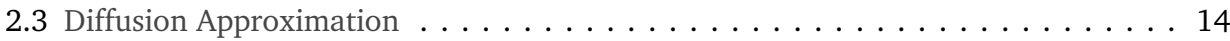

2.4 Simplified Spherical Harmonics . . . . . . . . . . . . . . . . . . 15

2.5 Finite Difference method $\ldots \ldots \ldots \ldots \ldots$. . . . . . . . . . . . . 16

2.6 Modal Method and Krylov Schur . . . . . . . . . . . . . . . . . . . 17

3 Neutron Transport Equation $\quad 23$

3.1 Background for Transport Theory . . . . . . . . . . . . . . . . . 23

3.2 The time dependent Neutron Transport Equation . . . . . . . . . . . . . . 35

3.3 1D steady state Neutron Transport Equation with Isotropic Scattering . . . . . . . . 41

3.4 1D steady state Neutron Transport Equation with Anisotropic Scattering. . . . . . . 46

3.5 2D steady state Neutron Transport Equation with Anisotropic Scattering. . . . . . . 52

3.6 Angular Quadratures . . . . . . . . . . . . . . . . . . . . . . . . . . 59

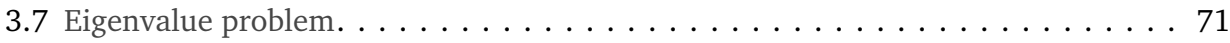

3.8 Numerical Results for Criticality Problems ․ . . . . . . . . . . . . . . . 75

3.9 Neutron Transport Fixed Source Problem . . . . . . . . . . . . . . . . . . . . . . . 94

3.10 Numerical Results for Fixed Source Problems ～. . . . . . . . . . . . . . 97

4 Neutron Diffusion Equation 103

4.1 Introduction to the Neutron Diffusion Equation . . . . . . . . . . . . . . . . 103

4.2 Derivation of the Neutron Diffusion Equation . . . . . . . . . . . . . . . . . . . 104

4.3 1D Finite Difference Diffusion Equation using Cell-Centered Scheme . . . . . . . . . 108

4.4 2D Finite Difference Diffusion Equation using Cell-Centered Scheme . . . . . . . . .118

4.5 1D Finite Difference Diffusion Equation using Edge-Centered Scheme . . . . . . . .123

4.6 Eigenvalue problem. . . . . . . . . . . . . . . . . . . . . . . 127

4.7 Numerical Results . . . . . . . . . . . . . . . . . . . . . . . . . 136

5 Simplified Spherical Harmonics 163

5.1 Introduction to the Simplified Spherical Harmonics . . . . . . . . . . . . . . . 163

5.2 Derivation of the Simplified Spherical Harmonics Equations. . . . . . . . . . . . . 165

$5.32 \mathrm{D}$ Cell centered scheme $S P_{3}$ Equations $\ldots \ldots \ldots \ldots \ldots \ldots \ldots \ldots \ldots$

5.4 Eigenvalue problem. . . . . . . . . . . . . . . . . . . . . 187

5.5 Numerical Results $1 \mathrm{D} S P_{3}$ equations. . . . . . . . . . . . . . . . . . . 191 
5.6 Numerical results for $2 \mathrm{D} S P_{3}$ equations . . . . . . . . . . . . . . . . 202

5.7 Numerical results for $3 \mathrm{D} S P_{3}$ equations $\ldots \ldots \ldots \ldots \ldots \ldots \ldots \ldots \ldots \ldots$

6 Conclusions 225

6.1 Conclusions . . . . . . . . . . . . . . . . . . . . . . . . .225

6.2 Scientific contributions. . . . . . . . . . . . . . . . . . . 228

6.3 Future works . . . . . . . . . . . . . . . . . . . . . . . . . 239

$\begin{array}{ll}\text { Bibliography } & 241\end{array}$ 



\section{List of Figures}

2.1 Energy discretization. . . . . . . . . . . . . . . 9

3.1 The spatial $(\vec{r})$, angular $(\hat{\Omega})$, and energy $(E)$ variables. . . . . . . 25

3.2 Thin neutron target. . . . . . . . . . . . . . . 25

3.3 Neutron probability. . . . . . . . . . . . . . . . . . . . 29

3.4 Typical fission spectrum plotted as a function of the neutron energy. 31

$3.5 d V$ about $\vec{r}, d \hat{\Omega}$ about $\hat{\Omega}$. . . . . . . . . . . . . . 32

3.6 Neutron flow through $d A$ and representation of the volume $\nu d t n \hat{\Omega} d A$. 34

3.7 Positive and negative directions of the neutron flow through $d A$. . 35

3.8 Neutron's phase space in the unit sphere. . . . . . . . . . . 36

3.9 Sphere reached by neutrons with direction $\hat{\Omega}$. . . . . . . . . 38

3.10 (3.10a) Time dependence of the flux for a source-free multiplying medium. (3.10b) Time dependence of the flux for a multiplying medium with a known source. . . . . . . . . . . . 43

3.11 Symmetry. . . . . . . . . . . . . . . . . 55

3.12 Spatial discretization. . . . . . . . . . . . . 57 
$3.132 \mathrm{D}$ angular directions for $S_{2} \ldots \ldots \ldots \ldots \ldots \ldots$

3.14 Vertical boundary condition with $1 \leq n \leq \frac{N}{2} \ldots \ldots \ldots$. . . . 58

3.15 Vertical boundary condition with $\frac{N}{2}+1 \leq n \leq N$. . . . . . . . 58

3.16 Horizontal boundary condition. . . . . . . . . . . . . . . . . 59

3.17 Corner boundary condition. . . . . . . . . . . . . . . . . . . . 59

3.18 Level Symmetric $S_{8}$ example. . . . . . . . . . . . . . . . . 63

3.19 Level-Symmetric quadratures for $S_{4}, S_{6}, S_{8}$ and $S_{16} \ldots \ldots$. . . 64

$3.20 P_{n}-E W$ quadratures for $S_{8} \ldots \ldots \ldots \ldots \ldots$

$3.21 P_{n}$-EW quadratures for $S_{4}, S_{6}, S_{8}$ and $S_{16} \ldots \ldots \ldots \ldots 6$

$3.22 P_{n}-T_{n}$ quadratures for $S_{4}, S_{6}, S_{8}$ and $S_{16} \ldots \ldots \ldots \ldots$

3.23 $P Q$ quadratures for $\left(\mathrm{n}^{\circ}\right.$ polar, $\mathrm{n}^{\circ}$ azim $)=(2,1),(1,2),(2,2),(3,2) \ldots 69$

3.24 $P Q$ quadratures for $\left(\mathrm{n}^{\circ}\right.$ polar, $\mathrm{n}^{\mathrm{o}}$ azim $)=(2,3),(3,3),(6,6),(8,8) \ldots \quad \ldots 70$

3.25 Two dimensional ordering scheme of the matrix $A$. . . . . . . . 71

3.26 Matrix $A, 1 \mathrm{D}$ (one dimension), $1 \mathrm{EG}$ (one energy group), $S_{8}$ (discrete ordinates order 8), 100 cells (discretization). . . . . . . . . . . 72

3.27 Matrix $A, 1 \mathrm{D}$ (one dimension), 2EG(two energy groups), $S_{8}$ (discrete ordinates order 8), 100 cells (discretization) and no upscattering (the upscattering is not considered). . . . . . . . . . . 72

3.28 Matrix A, 1D-2EG-S $S_{8}-100$ cells-upscattering. . . . . . . . . . 73

3.29 Matrix A, 2D-1EG- $S_{4}-10 x 10$ cells. . . . . . . . . . . . 73

3.30 Matrix A, 2D-2EG- $S_{4}$-10x10 cells-no upscattering. $\ldots \ldots \ldots \ldots$

3.31 Matrix A, 2D-2EG- $S_{4}$-10x10 cells-upscattering. . . . . . . . . 74

3.32 Geometry and cross sections for ISSA Benchmark. . . . . . . . . 76

3.33 Normalized scalar flux for ISSA Benchmark calculated with $n-$ DOTEC . . . . . . . . . . . . . . . 76

3.34 Cases for Seven Region Slab. . . . . . . . . . . . . . . . . . 77 
3.35 Scalar fluxes for each case of the 7 alternate region problem. . . . . 78

3.36 MOX benchmark problem geometry. . . . . . . . . . . . . 80

3.37 Scalar flux distribution for $2^{\text {nd }}$ eigenvalue. . . . . . . . . . . 82

3.38 Scalar flux distribution for $3^{\text {rd }}$ eigenvalue. . . . . . . . . . . 82

3.39 BWR cell problem geometry. . . . . . . . . . . . . . . 84

3.40 Spatial discretization influence. . . . . . . . . . . . . 84

3.41 Four dominant eigenvalues flux distribution for the BWR cell test problem. . . . . . . . . . . . . . 85

3.42 Material distribution and mesh for the BWR rod bundle test problem. 87

3.43 Four dominant eigenvalues flux distribution for the BWR rod bundle test problem. . . . . . . . . . . . . . 88

3.44 Assembly. . . . . . . . . . . . . . . . . . . . . . . . 89

3.45 Pin cell approximation. . . . . . . . . . . . . . . . . 89

3.46 composition layout. . . . . . . . . . . . . . . 90

3.47 composition layout. . . . . . . . . . . . . . . . . 91

3.48 Flux of the first mode for energy groups 1 and $7 . \ldots . . . .991$

3.49 Flux of the second mode for energy groups 1 and $7 \ldots$. . . . . . 92

3.50 Power Distribution of the C5G7 problem. . . . . . . . . . . . 92

3.51 One-dimensional source problem with vacuum boundary conditions. 98

3.52 Two-region source problem with reflective boundary conditions. . . 99

3.53 Two-group anisotropic scattering problem. . . . . . . . . . . 100

3.54 Two-dimensional problem. . . . . . . . . . . . . . . . . 102

4.1 Cell-centered finite difference scheme. . . . . . . . . . . . . . 113

4.2 Cell-centered finite difference scheme. . . . . . . . . . . . . . . 114

4.3 Finite difference edge-centered scheme. . . . . . . . . . . . . 123 
4.4 Edge-centered Scheme. . . . . . . . . . . . . . . . . . . 125

4.5 Left boundary conditions edge-centered scheme. . . . . . . . . . . 125

4.6 New two dimensional ordering scheme of the matrix $A$ for $S_{N}$ method. . . . . . . . . . . . . . . . 127

4.7 Matrix $A$, 1D-1EG- $S_{8}-100$ cells. . . . . . . . . . . . . . 128

4.8 Matrix A, 1D-1EG-S $S_{8}-100$ cells-zoom. . . . . . . . . . . . . 128

4.9 Matrix A, 1D-1EG-SP $P_{1}$ (Diffusion)-100 cells. . . . . . . . . . . . . 129

4.10 Matrix A, 1D-1EG-S $P_{1}$ (Diffusion)-100 cells-zoom. . . . . . . . . . 129

4.11 Matrix A, 1D-2EG- $S_{8}-100$ cells-no upscattering. . . . . . . . . . 130

4.12 Matrix A, 1D-2EG- $S_{8}-100$ cells-no upscattering -zoom. . . . . . . 130

4.13 Matrix A, 1D-2EG-S $P_{1}$ (Diffusion)-100 cells-no upscattering. . . . . 131

4.14 Matrix $A$, 1D-2EG- $S P_{1}$ (Diffusion)-100 cells-no upscattering -zoom. 131

4.15 Matrix A, 2D-2EG- $S_{4}-10 x 10$ cells-no upscattering. $\ldots \ldots \ldots . . .132$

4.16 Matrix A, 2D-2EG- $S_{4}-10 x 10$ cells-no upscattering-zoom1. $\ldots . . .132$

4.17 Matrix A, 2D-2EG- $S_{4}-10 x 10$ cells-no upscattering-zoom2. $\ldots \ldots$.

4.18 Matrix $A$, 2D-2EG- $S_{8}-10 x 10$ cells-no upscattering. $\ldots \ldots \ldots . . .133$

4.19 Matrix A, 2D-2EG- $S_{8}-10 x 10$ cells-no upscattering-zoom1. . . . . . 134

4.20 Matrix $A$, 2D-2EG- $S_{8}-10 x 10$ cells-no upscattering-zoom2. $\ldots . .134$

4.21 Matrix A, 2D-2EG- $S_{8}-10 x 10$ cells-no upscattering. $\ldots \ldots \ldots \ldots$

4.22 Matrix A, 2D-2EG- $S_{8}-10 x 10$ cells-no upscattering-zoom. . . . . . 135

4.23 Normalized Scalar Flux for Homogeneous 1D reactor. . . . . . . . . 137

4.24 Geometry and cross sections for ISSA Benchmark. . . . . . . . . . 138

4.25 ISSA: normalized scalar flux for the first mode. . . . . . . . . . . . 139

4.26 ISSA: normalized scalar flux for the second mode. . . . . . . . . . 139

4.27 ISSAe: normalized scalar flux for the third mode. . . . . . . . . . 140 
4.28 ISSA: normalized scalar flux for the fourth mode. . . . . . . . . . . 140

4.29 Cases for Heterogeneous slab problem. . . . . . . . . . . . . . . . . 141

4.30 Basecase: normalized scalar flux for the first mode. . . . . . . . . . 142

4.31 Basecase: normalized scalar flux for the second mode. . . . . . . . 143

4.32 Basecase: normalized scalar flux for the third mode. . . . . . . . . 143

4.33 Basecase: normalized scalar flux for the fourth mode. . . . . . . . . 144

4.34 Absorber Position 5: normalized scalar flux for the first mode. . . . 144

4.35 Absorber Position 6: normalized scalar flux for the first mode. . . . 145

4.36 MOX: First energy group scalar flux distribution for 1st mode. . . . 147

4.37 MOX: Profile comparison for the first energy group scalar flux distribution of the 1 st eigenvalue. . . . . . . . . . . . . . 148

4.38 MOX: Normalized Scalar flux distribution for $2^{\text {nd }}, 3^{\text {rd }}$ and $4^{\text {th }}$ modes, FDM diffusion on the left, FDM $S_{8}$ on the right. . . . . . . . . . 149

4.39 BWR cell problem geometry. . . . . . . . . . . . . . . . . 150

4.40 BWR cell test: scalar flux distribution for the $1^{\text {st }}$ mode, $1^{\text {st }}$ energy group. Diffusion and Discrete Ordinates, respectively. . . . . . . . . 151

4.41 BWR cell test: scalar flux distribution for the $1^{\text {st }}$ mode, $1^{\text {st }}$ energy group. . . . . . . . . . . . . . . . . . . . 152

4.42 Normalized scalar flux of the first four modes for the BWR cell test problem. FDM Diffusion on the left, FDM $S_{8}$ on the right. . . . . 153

4.43 BWR rod bundle mesh Mesh. . . . . . . . . . . . . . . . . . 154

4.44 BWR rod bundle test: scalar flux distribution for the 1st mode. . . . 155

4.45 BWR rod bundle test: scalar flux distribution for the 1st mode. . . . 156

4.46 Notmalized scalar flux distribution of the first four modes for the BWR rod bundle test problem. Diffusion on the left and discrete ordinates on the right. . . . . . . . . . . . . . . 157

4.47 Detail of $1 \times 1,4 \times 4,6 \times 6$ and $8 \times 8$ meshes. . . . . . . . . . . . 159 
4.48 FDM Diffusion Flux 1st eigenvalue for energy groups 1 (left) and 7 (right). . . . . . . . . . . . . . . . . 160

4.49 Power Distribution of C5G7 problem. . . . . . . . . . . . . 160

4.50 Eigenfluxes comparison for the four dominant eigenvalues of the $1^{\text {st }}$ energy group. FDM Diffusion on the left, FDM $S_{4}$ on the right. . 161

5.1 Cell-centered Finite Difference. . . . . . . . . . . . . . . . . . . 168

5.2 Cell-centered finite difference scheme. . . . . . . . . . . . . . . 173

5.3 Finite difference edge-centered scheme. . . . . . . . . . . . . 178

5.4 Edge-centered Scheme ～. . . . . . . . . . . . . . . . . . 180

5.5 Left boundary conditions edge-centered scheme. . . . . . . . . . 181

5.6 Matrix A, 1D-1EG- $S P_{3}-100$ cells. . . . . . . . . . . . . . . . 188

5.7 Matrix A, 1D-1EG- $S P_{3}-100$ cells-zoom. . . . . . . . . . . . . 188

5.8 Matrix A, 1D-2EG-SP $P_{3}-100$ cells. . . . . . . . . . . . . . . . 189

5.9 Matrix A, 1D-2EG-S $P_{3}-100$ cells-zoom. . . . . . . . . . . . . 189

5.10 Matrix A, 2D-2EG-SP$P_{3}-100$ cells. . . . . . . . . . . . . . . 190

5.11 Matrix A, 2D-2EG-SP$P_{3}-100$ cells-zoom. . . . . . . . . . . 190

5.12 Normalized Scalar Flux for Homogeneous 1D reactor. . . . . . . . . 193

5.13 Normalized Scalar Flux for Homogeneous 1D reactor and detail of the boundary. . . . . . . . . . . . . . . . . . . 194

5.14 Geometry and cross sections for ISSA Benchmark. . . . . . . . . . 195

5.15 ISSA: normalized scalar flux of the first mode. . . . . . . . . . . 196

5.16 ISSA: normalized scalar flux of the second mode. . . . . . . . . . . 196

5.17 ISSAe: normalized scalar flux of the third mode. . . . . . . . . . 197

5.18 ISSA: normalized scalar flux of the fourth mode. . . . . . . . . . 197

5.19 Cases for Heterogeneous slab problem. . . . . . . . . . . . . . 198 
5.20 Basecase: normalized scalar flux of the first mode. . . . . . . . . . 199

5.21 Basecase:normalized scalar flux of the second mode. . . . . . . . . 199

5.22 Basecase: normalized scalar flux of the third mode. . . . . . . . . . 200

5.23 Basecase: normalized scalar flux of the fourth mode. . . . . . . . . 200

5.24 Absorber Position 5: normalized scalar flux of the first mode. . . . . 201

5.25 Absorber Position 6: normalized scalar flux of the first mode. . . . . 201

5.26 MOX benchmark problem geometry. . . . . . . . . . . . . . 202

5.27 MOX: First energy group scalar flux distribution for first mode. . . . 204

5.28 MOX: Normalized Scalar flux distribution for $2^{\text {nd }}, 3^{\text {rd }}$ and $4^{\text {th }}$ modes, FDM $S P_{3}^{C}$ on the left, FDM $S_{8}$ on the right. . . . . . . 205

5.29 BWR cell problem geometry. . . . . . . . . . . . . . . . 206

5.30 BWR cell test: normalized scalar flux distribution for $1^{\text {st }}$ mode, $1^{\text {st }}$ energy group. . . . . . . . . . . . . . . . 207

5.31 Four modes normalized first group flux distribution for the BWR cell test problem. FDM $S P_{3}$ on the left, FDM $S_{8}$ on the right. . . . 208

5.32 BWR rod bundle test material distribution and mesh. . . . . . . . . 209

5.33 BWR rod bundle test: scalar flux distribution for 1st mode by using cell-centered scheme. . . . . . . . . . . . . . . . . . . . . 210

5.34 Four modes flux distribution for the BWR rod bundle test problem. 211

5.35 Detail of $1 \times 1,4 \times 4,6 \times 6$ and $8 \times 8$ meshes. . . . . . . . . . . . 213

$5.36 \mathrm{FDM} S P_{3}$ Flux 1st mode for energy groups 1 (left) and 7 (right). . 214

5.37 Power Distribution of C5G7 problem. . . . . . . . . . . . . . 214

5.38 Eigenfluxes comparison for the four dominant modes of the $1^{\text {st }}$ energy group. FDM $S P_{3}$ on the left, FDM $S_{4}$ on the right. . . . 215

5.39 Scalar flux first mode $S_{2}$ C5G7 complete. . . . . . . . . . . 217

5.40 Homogeneous reactor meshes. . . . . . . . . . . . . . . 218 
5.41 Homogeneous reactor flux distribution for eigenvalue 1 . . . . . 219

5.42 Normalized Scalar Flux for Homogeneous 3D reactor for a central

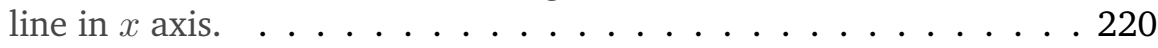

5.43 Normalized Scalar Flux for Homogeneous 3D reactor for a central line in $y$ axis. . . . . . . . . . . . . . . . . 221

5.44 Normalized Scalar Flux for Homogeneous 3D reactor for a central line in $\mathrm{z}$ axis. . . . . . . . . . . . . . . . . . 2221

5.45 FBR reactor discretization (interior visualization of the reactor upper part). . . . . . . . . . . . . . . . . 222

5.46 Case 1: FBR reactor flux distribution for group 1. . . . . . . . 223

5.47 Case 2: FBR reactor flux distribution for group $1 \ldots \ldots$. . . . . . 223

5.48 Case 1: FBR reactor flux distribution for group 4. . . . . . . . . 224

5.49 Case 2: FBR reactor flux distribution for group 4. . . . . . . . . 224 


\section{List of Tables}

2.1 Iterative methods and preconditioners . . . . . . . . 20

3.1 Gain and loss mechanisms . . . . . . . . . . . 37

3.2 Cross-Sections for Seven Region Slab. . . . . . . . . . . . . 77

3.3 Seven-region eigenvalues. . . . . . . . . . . . . . . 78

3.4 Analytical Benchmark Results. $p c m=(\mid$ ref.value-value $\mid /$ ref.value $)$. $10^{5} * A B=$ Analytical Benchmarck [Sood et al., 2003] . . . . . . 79

3.5 MOX benchmark problem cross-sections. $\mathrm{g}=1$ (fast energy group), $\mathrm{g}=2$ (thermal energy group). . . . . . . . . . . . 81

3.6 Dominant eigenvalues for the MOX problem. SHNC=Spherical Harmonics Nodal Collocation. . . . . . . . . . . . . . . . . . 81

3.7 BWR cell benchmark problem cross-sections. $\mathrm{g}=1$ (fast energy group), $g=2$ (thermal energy group). . . . . . . . . . 83

3.8 BWR cell benchmark problem results for different quadrature or-

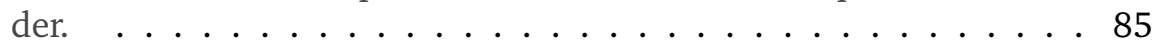

3.9 BWR rod bundle benchmark problem cross-sections. . . . . . . . 86

3.10 BWR rod bundle benchmark problem multiplication factor results for different quadrature order. . . . . . . . . . . 87 
3.11 Sub-critical modes comparison. ${ }^{1}$ SHNC $=$ Spherical Harmonics Nodal Collocation. . . . . . . . . . . . . . . . . 90

3.12 C5G7 Test problem results. . . . . . . . . . . . . . . 93

3.13 Scalar fluxes for the homogeneous slab with vacuum boundary conditions and 10 spatial cells for different $S_{N} \ldots \ldots \ldots 98$

3.14 Scalar fluxes for the homogeneous slab with vacuum boundary conditions and 100 spatial cells for different $S_{N}$. (* corresponds to calculation with 1000 spatial cells). . . . . . . . . . . . 98

3.15 Scalar fluxes for the two-region source problem with reflective boundary conditions and 10 spatial cells for different $S_{N}$. . . 99

3.16 Scalar fluxes for the two-region source problem with reflective boundary conditions and 100 spatial cells for different $S_{N}$ (* corresponds to calculation with 200 spatial cells). . . . . . . . . . 99

3.17 Scalar fluxes for the first group of the anisotropic problem using different $S_{N} \ldots \ldots \ldots \ldots \ldots \ldots \ldots \ldots \ldots \ldots \ldots \ldots \ldots \ldots$

3.18 Scalar fluxes for the second group of the anisotropic problem using different $S_{N} \ldots \ldots \ldots \ldots \ldots 10 \ldots \ldots \ldots$

3.19 Scalar fluxes along $\mathrm{y}=5$ for the two-dimensional problem with $26 \times 26$ and $52 \times 52$ cells discretization for different $S_{N}$ orders. . . . 102

4.1 First 4 eigenvalues - homogeneous slab reactor. (Dif. ${ }^{C}$ is the diffusion code using cell-centered sheme). (Dif. ${ }^{E}$ is the diffusion code using edge-centered Scheme). . . . . . . . . . . . . . . . . 137

4.2 ISSA problem eigenvalues. . . . . . . . . . . . . 138

4.3 Cross-Sections for Heterogeneous slab problem. . . . . . . . . . . . 141

4.4 Heterogeneous slab problem eigenvalues. . . . . . . . . . . . . 142

4.5 Dominant eigenvalues for the MOX problem. *SHNC=Spherical Harmonics Nodal Collocation. . . . . . . . . . . . . . . . . . . 148

4.6 BWR cell benchmark problem multiplication factor results. . . . . 150

4.7 Eigenvalues comparison. ${ }^{1}$ SHNC $=$ Spherical Harmonics Nodal Collocation Capilla et al. [2018]. . . . . . . . . . . . . . . . 154 
4.8 Eigenvalues comparison. ${ }^{1}$ SHNC $=$ Spherical Harmonics Nodal Collocation Capilla et al. [2018]. . . . . . . . . . . . . . . . . . 158

$4.9 k_{e f f}$ and power comparison by using cell-centered Scheme. . . . . 159

$4.10 K_{\text {eff }}$ and power comparison by using edge-centered scheme. . . . 160

4.11 C5G7 Test problem results. *Results from [Vidal-Ferràndiz et al., 2019]. . . . . . . . . . . . . . . . . 162

5.1 Values of the first four eigenvalues for a homogeneous slab $2 \mathrm{~cm}$ thick. (Dif. ${ }^{C}$ is the $S P_{1}$ code using cell-centered scheme). (Dif. ${ }^{E}$ is the $S P_{1}$ code using edge-centered scheme) . . . . . . . . . . . 192

5.2 Values of the first four eigenvalues for a homogeneous slab $2 \mathrm{~cm}$ thick. ( $S P_{3}^{C}$ is the $S P_{3}$ code using cell-centered scheme). ( $S P_{3}^{E}$ is the $S P_{3}$ code edge-centered scheme). . . . . . . . . . . . . . 192

5.3 Values of the first four eigenvalues for a homogeneous slab $2 \mathrm{~cm}$ thick. ( $S P_{3}^{C *}$ is the $S P_{3}$ code using cell-centered scheme and $B C$ refers to each boundary condition approach). . . . . . . . . . . . 193

5.4 ISSA problem eigenvalues. . . . . . . . . . . . . . 195

5.5 Cross-Sections for heterogeneous slab problem. . . . . . . . . . . 198

5.6 Heterogeneous slab problem eigenvalues. . . . . . . . . . . . 198

5.7 MOX benchmark problem cross-sections. $g=1$ (fast energy group), $\mathrm{g}=2$ (thermal energy group). . . . . . . . . . . . . . 203

5.8 Dominant eigenvalues for the MOX problem. *SHNC=Spherical Harmonics Nodal Collocation. . . . . . . . . . . . . . . . . . 203

5.9 BWR cell benchmark problem multiplication factor results. . . 207

5.10 BWR rod bundle benchmark problem multiplication factor results. 209

$5.11 k_{\text {eff }}$ and power comparison for the cell-centered Scheme. . . . . . 212

$5.12 k_{\text {eff }}$ and Power comparison for the edge-centered scheme. . . . . . 213

5.13 C5G7 Test problem results. *Results from [Vidal-Ferràndiz et al., 2019]. . . . . . . . . . . . . . . . . 216 
5.14 Computational times. . . . . . . . . . . . . . . . . . 217

5.15 Homogeneous problem cross-sections. $\mathrm{g}=1$ (fast energy group), $\mathrm{g}=2$ (thermal energy group). . . . . . . . . . . . . 218

5.16 Multiplication factors for Homogeneous 3D problem. . . . . . . 219

5.17 Values of the first four modes for a 3D homogeneous problem, SP3 boundary condition approaches comparison. $\left(\mathrm{SP}_{3}^{C}\right.$ is the $\mathrm{SP}_{3}$ code using cell-centered scheme and $B C$ refers to each boundary condition approach). . . . . . . . . . . . . . . . . 220

5.18 Multiplication factors for FBR problem. . . . . . . . . . . 222 


\title{
List of Symbols
}

\author{
Acronyms
}

AVG Average Error

BWR Boiling Water Reactor

DSA Diffusion Synthetic Acceleration

FDM Finite Difference Method

FEM Finite Element Method

FVM Finite Volume Method

GMRES Generalized Minimal Residual Method

ILU Incomplete LU descomposition

LU Lower Upper descomposition

MOC Method of Characteristics

MPE Maximum Percentage Error

MRE Mean Relative Error

MUMPS Multifrontal Massively Parallel Sparse direct solver

NCM Nodal Collocation Method

PETSc Portable, Extensible Toolkit for Scientific computations 
PWR Pressurized Water Reactor

RMS Root Mean Square

SHNC Spherical Harmonics Nodal Collocation

SLEPc Scalable Library for Eigenvalue Problem computations

WCD Weight Central Difference

\section{Symbols}

$\beta \quad$ Albedo condition

$\chi \quad$ Fission spectrum

$\Delta k_{\text {eff }}$ Multiplicative factor error in (pcm)

$\Delta \lambda \quad$ Eigenvalue error in $(\mathrm{pcm})$

$\hat{\Omega} \quad$ Direction of the neutron

$\hat{\Omega}_{n} \quad$ Discrete ordinate $n$

$\lambda \quad$ Eigenvalue

$\mu \quad$ Cosine of angle of the neutron velocity

$\mu_{0} \quad$ Cosine of angle scattering

$\nabla \quad$ Nabla operator

$\nabla^{2} \quad$ Laplacian operator

$\nu \quad$ Mean number of born neutron per fission

$\omega$ or $\varphi$ Azimuthal angle

$\omega_{n} \quad$ Weighting factor for discrete ordinate $n$

$\phi \quad$ Scalar neutron flux

$\phi_{g} \quad$ Scalar neutron flux of group $g$

$\psi \quad$ Angular neutron flux

$\psi_{g}^{m} \quad$ Neutron flux of the $m^{\text {th }}$ order moment in group $g$

$\psi_{l} \quad$ Legendre moment for the flux 
$\sigma \quad$ Microscopic cross section

$\Sigma_{a} \quad$ Macroscopic absorption cross section

$\Sigma_{f} \quad$ Macroscopic fission cross section

$\Sigma_{r} \quad$ Macroscopic removal cross section

$\Sigma_{s} \quad$ Macroscopic scattering cross section

$\Sigma_{t} \quad$ Macroscopic total cross section

$\Sigma_{s, g^{\prime} \rightarrow g, l}$ Legendre moment for the scattering

$\Sigma_{s}\left(\vec{r}, E^{\prime} \rightarrow E, \hat{\Omega}^{\prime} \rightarrow \hat{\Omega}\right)$ Macroscopic scattering cross section from energy $E^{\prime}$ to $E$ and from direction $\hat{\Omega}^{\prime}$ to $\hat{\Omega}$

$\theta \quad$ Polar angle

$\vec{J} \quad$ Net current vector

$\vec{n} \quad$ Normal vector

$\vec{r} \quad$ Spatial position coordinates

$\widetilde{D} \quad$ Coupled diffusion coefficient

$D \quad$ Diffusion coefficient

$D i f f^{C}$ Diffusion cell-centered scheme code

Diff ${ }^{E}$ Diffusion edge-centered scheme code

$E \quad$ Energy of the neutron

$E_{\max }$ Maximum relative error

$E_{r} \quad$ Relative error

$E O_{N}$ Even-odd Moment Quadrature

$f(E)$ Energy dependent spectral weighting function

$G \quad$ Number of energy groups

$g \quad$ Discretized Energy group

$h_{i} \quad$ Length of the cell $i$ in axis $x$ 
$I \quad$ Maximum distance in axis $x$

$i \quad$ Mesh cell number or node position

$J \quad$ Maximum distance in axis $y$

$J^{ \pm} \quad$ Neutron partial current

$J_{n} \quad$ Net current through direction $n$

$J_{n}^{ \pm} \quad$ Partial currents through direction $n$

$k \quad$ Eigenvalue or mode

$k_{\text {eff }} \quad$ Multiplicative factor or fundamental eigenvalue

$k_{j} \quad$ Length of the cell $j$ in axis $y$

$L \quad$ Order of the Legendre polynomial

$L S$ or $L Q_{N}$ Level Symmetric Quadrature

$M \quad$ Quadrature order

$N \quad$ Neutron density probability distribution

$N \quad$ Quadrature order

$P_{l} \quad$ Legendre polynomial of order $l$

$P_{l}^{k} \quad$ Associated Legendre polynomials

$P_{N} \quad$ Spherical Harmonic Method of order N

$P_{N}-T_{N}$ Legendre-Chebyshev Quadrature

$P Q \quad$ Product Quadrature

$Q \quad$ Source term

$S_{N} \quad$ Discrete Ordinates Method of order N

$S_{3}^{C} \quad$ Diffusion cell-centered scheme code

$S P_{3}^{E} \quad$ Diffusion edge-centered scheme code

$S P_{N} \quad$ Simplified Spherical Harmonic Method of order N

$T_{n} \quad$ Chebyshev polynomial

$U E_{N}$ or $U G_{N}$ Positive Weight Quadrature

$Y_{n, m}(\hat{\Omega})$ Spherical Harmonics functions 




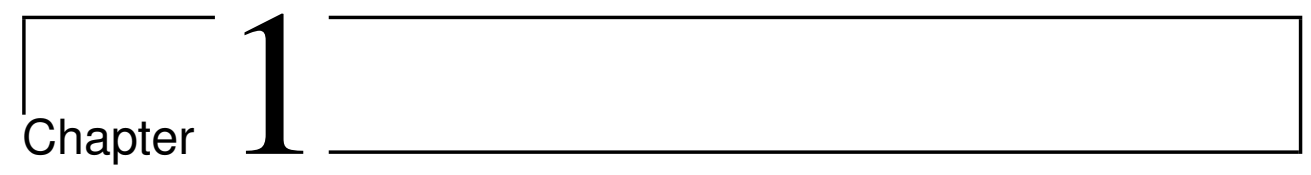

\section{Introduction}

\subsection{Motivation and Objectives}

The power generated inside nuclear reactors is one of the most important parameters in Nuclear Safety Analyses. The energy is released by the nuclear fissions inside the reactor core, which is proportional to the neutron flux. Therefore, the calculation of the neutron flux distribution can determine the spatial and time distribution of the power.

Then, the analysis and design of nuclear reactors are based on the neutron distribution in the system and the most accurate manner of calculating the neutron population is by solving the Neutron Transport Equation. This equation depends on the spatial variables, neutron energy, angular direction and time. Although, the real number of neutrons per unit volume is continuously varying with time, even in steady-state conditions, under this assumption, the neutron number density oscillates about an average value related to the solution of the steady state of the neutron transport equation. Many fields of nuclear engineering make use of a solution of the neutron transport equation, such as, reactor physics, nuclear safety, criticality, radiation shielding and radiological protection.

Furthermore, the use of the neutron transport equation is not only limited to the power plants or reactor core calculations. The use of the neutron transport equation in fixed source problems is very common in the medical field to dose distribution calculation for radiation protection of the patients. 
In any case, the spatial distribution of the neutron flux can be determined by solving the steady state of the neutron transport equation. Analytical solutions are available in very limited cases, thus, it is necessary to apply numerical methods and discretize the variables appearing in the equation.

Different approximations to the neutron transport equation have been developed during decades. The Diffusion approximation is widely used in the analysis and design of nuclear reactors, which allows core calculations with reasonable computational time and accuracy. However, more and more rigorous approximations are required for solving those problems in which the diffusion approximation simply is not valid, due to the heterogeneities of the materials, for example to perform detailed reactor core calculations or in the medical field where the human body presents a lot of heterogeneities when the particles pass from the soft tissue to the bones. In this regard, one of the purpose of this thesis is to show the differences in accuracy of different approximations to the neutron transport equation and the advantages or disadvantages of each method. Hence, three different approaches are introduced in this thesis: the Discrete Ordinates neutron transport equation, the Diffusion approximation and the Simplified Spherical Harmonics equations.

Several numerical methods can be applied to solve the different approaches to the neutron transport equation. Modal method was selected in this work to solve eigenvalue problems defined by the three methods previously described.

Therefore, the main objectives of this thesis can be summarized as follows:

1. To develop a Finite Difference Method for the Steady State Discrete Ordinates Neutron Transport Equation in order to solve criticality and fixed source problems developing several angular quadrature algorithms and considering both, isotropic and anisotropic scattering problems.

2. To implement a Finite Difference Method for the Steady State Diffusion Equation using two different approaches of the Finite Difference method.

3. To develop a Finite Difference Method for the Steady State Simplified Spherical Harmonics Equations, using two different approaches of the Finite Difference methods and review the boundary conditions applied to each of them.

4. To compare all the developed algorithms studying their accuracy for several simple and complex problems. 


\subsection{Thesis outline}

This thesis is composed of six chapters. The following chapter is the state of the art which introduces the principal subjects related with the thesis. This chapter presents an overview of the methods used in this thesis, as well as, a review of the codes and authors who worked in these methods previously.

Chapter 3 is a thorough study of the Neutron Transport Equations, taking as a starter point the definition and explanation of the parameters needed to understand the derivation of the multidimensional anisotropic steady state neutron transport equation. The discretization methods to solve this transport equation are presented in detail, Finite Difference Method and Discrete Ordinates method with a brief review of the angular quadratures implemented. At the end of this chapter the numerical results are exposed for different type of problems which validate the code developed based on the methods explained at the beginning of the chapter.

Chapter 4 follows similar structure. This chapter is devoted to the Diffusion Aproximation. First sections derive the Diffusion equation from the Neutron Transport Equation. The following sections explain the discretized Diffusion equation using different type of Finite Difference Methods and at the end of the chapter the numerical results are shown.

Once again, chapter 5 continues with the same text structure. First is explained the Simplified Spherical Harmonics Equations. Next, the derivation of the equations with the two different Finite Difference schemes is presented. At the end of the chapter a numerical results comparison is shown for all the methods explained, including the methods from the previous chapters.

Finally, first section of chapter 6 summarize the conclusions extracted from the previous chapter of the thesis. Section 6.2 is a list of the contributions provided by the author during the time that the doctoral studies lasted and section 6.3 set the points of departure for future works as a follow-up of this thesis. 



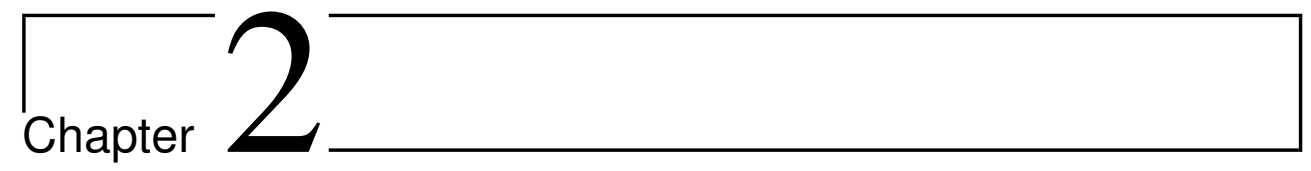

\section{State of the art}

\subsection{The Transport Equation}

The definition of the "transport theory" can be expressed as the mathematical description of the transport of some variable trough matter or a medium. There are many fields where this definition make sense. For example, to describe the movement of the neutrons through the uranium fuel inside a nuclear reactor, the diffusion of the light (photons) trough matter, or even the motion of gas molecules. So, transport theory has been widely studied in many fields of physics and engineering.

The beginnings of the transport theory go back more than one hundred years to the Boltzmann equation, formulated with the purpose of studying the kinetic theory of gases. Some time later, analytical solutions of the radiations transport problems in the stellar field appeared in the 1930s. Astrophysical studies stimulated knowledge about transport theory with the purpose of knowing the stellar radiant energy transfer. This gave way to the description of neutron and gamma transport in nuclear field. It was not until 1940s, when the scientific community paid attention to solve problems related with the reactor core configurations and radiation shielding applications.

However, the beginning of the "nuclear era" is attributed to the decade of the fifties when the problem of simulating the interactions of the neutrons with matter becomes important. This is because at the end of the second world war nuclear engineering focused the effort in using nuclear reactions to generate electric 
power or for different applications in the medical field. Then, after the cold war, started the construction of nuclear reactors to generate an important fraction of electricity used by humankind. Unfortunately, despite the benefits of this kind of power generation due to the fact that this technology is free of greenhouses gases emission, or the use of the medical applications of the nuclear technology like radiotherapy to treat cancer, there is still the association of the nuclear energy with the massive destruction weapons.

Getting back to the matter at hand, in the last decades have been implemented more accurate and sophisticate numerical methods to solve the Neutron Transport problem, at the same time that the computational power was increasing thanks to the development in processors.

From sixties until today, several numerical methods and extended documentation about them can be found, such as, [Davison et al., 1958], [Osborn and Yip, 1966], [Carlson, 1968], [Bell and Glasstone, 1970], [Henry et al., 1975], [Duderstadt and Hamilton, 1976a], [Duderstadt and Martin, 1979], [Lewis and Miller Jr, 1984] and more recently, [Stacey, 2007],[Lewis, 2008], [Cacuci, 2010], [Azmy and Sartori, 2010] and [Hébert, 2009].

A typical neutron transport problem is defined by a physical system $V$ and a neutron source, which can be internal like occurs with fission events or external when the neutrons reach the outer surface of a system. Hence, different interactions can appear between the neutrons traveling inside the system and the matter. This events can be: captures, scatterings or fissions events. The desired information from solving this problem is the neutron flux distribution within the system.

Then, the mathematical expression used for the steady-state problem can be described by the linear Boltzmann equation for the angular flux, [Larsen, 2006]:

$$
\begin{aligned}
\hat{\Omega} \cdot \nabla \psi(\vec{r}, \hat{\Omega}, E)+\Sigma_{t}(\vec{r}, E) \psi(\vec{r}, \hat{\Omega}, E)= \\
\quad \int_{0}^{\infty} \int_{4 \pi} \Sigma_{s}\left(\vec{r}, \hat{\Omega}^{\prime} \rightarrow \hat{\Omega}, E^{\prime} \rightarrow E\right) \psi\left(\vec{r}, \hat{\Omega}^{\prime}, E^{\prime}\right) d \hat{\Omega}^{\prime} d E^{\prime} \\
+\frac{\chi(\vec{r}, E)}{4 \pi} \int_{0}^{\infty} \int_{4 \pi} \nu \Sigma_{f}\left(\vec{r}, E^{\prime}\right) \psi\left(\vec{r}, \hat{\Omega}^{\prime}, E^{\prime}\right) d \hat{\Omega}^{\prime} d E^{\prime}+\frac{Q(\vec{r}, E)}{4 \pi}
\end{aligned}
$$

Where the independent variables are the 3-D spatial variable $\vec{r}=(x, y, z)$, the 2-D angular variable $\hat{\Omega}$, and energy $E$. Other parameters are: $\Sigma_{t}(\vec{r}, E)$, which is the total cross section, $\Sigma_{s}\left(\vec{r}, \hat{\Omega}^{\prime} \rightarrow \hat{\Omega}\right.$ is the differential scattering cross section, $\Sigma_{f}\left(\vec{r}, E^{\prime}\right) \psi\left(\vec{r}, \hat{\Omega}^{\prime}, E^{\prime}\right)$ defines the fission cross section whose fission spectrum 
is $\chi(\vec{r}, E)$ and the isotropic interior source is $Q(\vec{r}, E)$. Every term mentioned is well explained in chapter 3 where the neutron transport equation is deeply studied.

Most useful transport problems can be classified into three categories:

- Eigenvalue Calculations $\rightarrow$ In this cases the eigenvalue $k$ which is the reactor criticality parameter is introduced in the denominator of the fission term and the entire reactor is considered as the system. So, eq.2.1 remains:

$$
\begin{aligned}
& \hat{\Omega} \cdot \nabla \psi(\vec{r}, \hat{\Omega}, E)+\Sigma_{t}(\vec{r}, E) \psi(\vec{r}, \hat{\Omega}, E)= \\
& \quad \int_{0}^{\infty} \int_{4 \pi} \Sigma_{s}\left(\vec{r}, \hat{\Omega}^{\prime} \rightarrow \hat{\Omega}, E^{\prime} \rightarrow E\right) \psi\left(\vec{r}, \hat{\Omega}^{\prime}, E^{\prime}\right) d \hat{\Omega}^{\prime} d E^{\prime} \\
& \quad+\frac{1}{k} \frac{\chi(\vec{r}, E)}{4 \pi} \int_{0}^{\infty} \int_{4 \pi} \nu \Sigma_{f}\left(\vec{r}, E^{\prime}\right) \psi\left(\vec{r}, \hat{\Omega}^{\prime}, E^{\prime}\right) d \hat{\Omega}^{\prime} d E^{\prime}
\end{aligned}
$$

The intent of the $k$ is to equilibrate the leakage and creation of neutrons in the system with the purpose of obtaining an exact balance. This allows an steady-state solution of the flux. If $k>1$ a supercritical system is obtained and it is necessary to suppress fission events to return to the equilibrium. Contrarily, when $k<1$ fission events are enhanced to arrive again to the steady-state. The reactor is balanced when $k=1$. This is one of the most important problems to solve in nuclear reactor operation and design.

- Assembly calculations $\rightarrow$ This kind of problems considers only a certain part of the reactor called assembly. The $k$ continues appearing in this problem, but in this case, it is not the criticality of the reactor. The aim of this problem is to obtain the neutron flux distribution inside a single assembly because with this flux distribution and considering that all the assemblies are identical, then this calculated $k$ would be equal to the criticality $k$ of the complete reactor under the assumption of a infinite reactor.

- Shielding Calculations $\rightarrow$ These problems consider the reactor shield as the system. The fission term is set to zero because no fissionable material exists in the shield. The purpose of solving this problem is to know how many particles (photons or neutrons) the shielding material absorbs, and to calculate how much radiation passes through the shield. Moreover, the Fixed Source Problem could be included in this category, which is not only considered for reactor problems. 


$$
\begin{aligned}
& \hat{\Omega} \cdot \nabla \psi(\vec{r}, \hat{\Omega}, E)+\Sigma_{t}(\vec{r}, E) \psi(\vec{r}, \hat{\Omega}, E)= \\
& \int_{0}^{\infty} \int_{4 \pi} \Sigma_{s}\left(\vec{r}, \hat{\Omega}^{\prime} \rightarrow \hat{\Omega}, E^{\prime} \rightarrow E\right) \psi\left(\vec{r}, \hat{\Omega}^{\prime}, E^{\prime}\right) d \hat{\Omega}^{\prime} d E^{\prime}
\end{aligned}
$$

In practice, it is not common to solve entire commercial reactors, including shield, because this generates large and complex numerical problems. Instead of considering the complete reactor, more efficient problems using smaller parts of the reactor like assemblies or shieldings, are normally carried out. Finally, the individual results are combined in a correct way to obtain an accurate solution.

The principal techniques developed to solve the problems mentioned above can be classified as stochastic and deterministic. The most known stochastic approach is the Monte Carlo method. As stated in [Larsen, 2006], "Although these two approaches simulate the same physical problem, they are fundamentally different in terms of their approach to the problem and their solution techniques, have been developed by distinct computer code groups, exist only in separate computer codes, and have complementary advantages and disadvantages".

A brief explanation that just scratch the meaning of the last sentence is the fact that the neutron transport theory has a contradiction. The neutron path is a sequence of random events. On the other hand, the solution of the neutron transport equation is not random. Therefore, although Monte Carlo method describes the mean neutron flux simulating a huge number of histories or neutron paths, it ignores the mathematical description of the problem. In contrast, deterministic solution ignores the random aspects of the possible neutron paths. Another detail is that if one was able to simulate infinite number of histories it would converge to same solution of solved neutron transport equation. Obviously, since that is not possible, a statistic error is provide by the Monte Carlo technique.

It is not the purpose of this thesis to compare the advantages or disadvantages of this two different techniques, but the author would like to make a comment in this matter. Although Monte Carlo does not require any kind of approximation, since it uses an easy way of simulation because Monte Carlo directly applies the physics of particle transport to each individual particle without any truncation error, its simulations have high computational costs to have an acceptable statistical error.

Nowadays, there are some researchers working in codes that combine both techniques in a Hybrid Monte Carlo-Deterministic code, that uses the adjoint solution of the deterministic code to obtain biasing parameter and subsequently, the bias- 
ing parameters are used in the Monte Carlo simulation [Wu et al., 2012], [Larsen] and [Zhuang et al., 2019].

With respect to the deterministic techniques, many methods have been developed to solve the neutron transport equation. The first step of these methods is to discretize the independent variables. The variable that normally uses the same discretization independently of the method is the neutron energy. Considering that the particles have energies $E$ inside the range $E_{\min }<E<E_{\max }$, the energy discretization consists in dividing this interval into a determined number of $G$ energy groups as shown in fig.2.1.

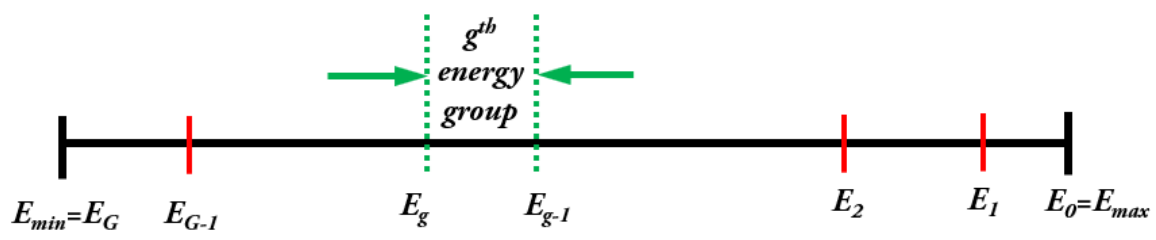

Figure 2.1: Energy discretization.

If all the cross sections are also defined in different energy groups, having one value with in each interval the Multi-group Neutron Transport Equation is defined as:

$$
\begin{aligned}
\hat{\Omega} \cdot \nabla \psi_{g}(\vec{r}, \hat{\Omega})+ & \Sigma_{t, g}(\vec{r}) \psi_{g}(\vec{r}, \hat{\Omega})= \\
& \sum_{g^{\prime}=1}^{G} \int_{4 \pi} \Sigma_{s, g^{\prime} \rightarrow g}\left(\vec{r}, \hat{\Omega}^{\prime} \rightarrow \hat{\Omega}\right) \psi_{g^{\prime}}\left(\vec{r}, \hat{\Omega}^{\prime}\right) d \hat{\Omega}^{\prime} \\
& +\frac{\chi_{g}(\vec{r})}{4 \pi} \sum_{g^{\prime}=1}^{G} \int_{4 \pi} \nu \Sigma_{f, g^{\prime}}(\vec{r}) \psi_{g^{\prime}}\left(\vec{r}, \hat{\Omega}^{\prime}\right) d \hat{\Omega}^{\prime}+\frac{Q_{g}(\vec{r})}{4 \pi}
\end{aligned}
$$

Mainly, the difference between the deterministic methods lies in the different angular approach that each one performs.

In any case, this thesis is focused in three different deterministic methods: the Discrete Ordinates method, the Diffusion approximation and the Simplified Spherical Harmonics method. The most common methods to solve the neutron transport equation are the Spherical Harmonics $P_{N}$ method and the Discrete Ordinates $S_{N}$ method. 
The $P_{N}$ method uses an expansion of the angular flux in a finite number of spherical harmonics functions, 2.5. Also, multiplying 2.4 by the spherical harmonics functions $Y_{n, m}(\hat{\Omega})$ and integrating $\hat{\Omega}$ over the unit sphere, one can obtain a coupled system of equations.

$$
\psi_{g}(\vec{r}, \hat{\Omega}) \approx \sum_{n=0}^{N} \sum_{m=-n}^{+n} \psi_{g, n, m}(\vec{r}) Y_{n, m}(\hat{\Omega})
$$

However, this thesis is focused on the Discrete Ordinates $S_{N}$ method which is widely used. The system of equations obtained by $S_{N}$ method is very similar to the original Boltzmann equation while the system obtained by $P_{N}$ equations have a very different and complicated mathematical structure.

There are not several codes that make use of the $P_{N}$ formulation, some examples of them are EVENT [Ziver et al., 2005], FESH [Blomquist, 1979], TEPFEM [Cao and $\mathrm{Wu}, 2004$ ] or the code developed by [Capilla et al., 2008]. $S_{N}$ method is more commonly used and it can be found implemented in codes such as, DORT/TORT [Rhoades and Childs, 1993], DANTSYS [Alcouffe et al., 1995], PENTRAN [Sjoden and Haghighat, 1997] or more recently using masive parallelization, PARTISN [Alcouffe et al., 2005], DRAGON [Marleau et al., 2008], ARES [Chen et al., 2017], DENOVO [Evans et al., 2010], ATTILA [Youssef et al., 2013] and TITAN [Yi, 2009].

Other common deterministic method that solves the neutron transport equation is the Method of Characteristics (MOC), [Askew, 1972]. The main advantage of this method is that it can provide solutions for any kind of mesh shape. MOC use a polar and azimuthal angles discretization and integrates the characteristic form of the equation for a particular azimuthal and polar angle quadrature. This method was first implemented in CACTUS, [Halsall, 1980]. Recent years, several research codes have been developed using MOC, some of them are MPACT code developed at the University of Michigan [Collins et al., 2013], nTRACER code from Seoul National University [Jung, 2010] and DRAGON which was developed at École Polytechnique de Montréal [Marleau et al., 2008].

Furthermore, there exist other approximations to the neutrons transport equation which do not solve directly the neutron transport equation but they solves equations derived from it after some simplifications. These equations are the Diffusion equation and the Simplified Spherical Harmonics $S P_{N}$ which will be deeply studied in chapters 4 and 5 , respectively. 
Great number of codes were developed, due to the fact that Diffusion is the more computational efficient method. Some of them are SIMULATE [Bahadir et al., 2005], PARCS [Downar et al., 2004], VALKIN [Verdú et al., 2002], VALKINFVM [Bernal et al., 2018a], NESTLE [Turinsky et al., 1994] or COBAYA [GarcíaHerranz et al., 2017].

Currently, different methods based on $S P_{n}$ are flourishing. For example, MPACT uses the one-dimensional $S P_{3}$ method in the axial direction and two-dimensional MOC in radial direction to solve 3D problems [Collins et al., 2016], the FEMFFUSION code [Vidal-Ferràndiz et al., 2018b] and PARCS [Lee et al., 2015].

Next sections of this chapter are devoted to introducing the methods used in this thesis which will be deeply studied in subsequent chapters.

\subsection{Discrete Ordinates Method}

The Discrete Ordinates deterministic method was originally developed for the study of cosmic radiation. The main theory about the Discrete Ordinates method $S_{N}$ adapted for neutron transport was developed by Bengt Carlson and Kaye Lathrop in 1968, [Carlson, 1968]. At that time, it was used only for reactor physics simulations and due to the few computation power that allows the first computers, the capabilities of the method were very limited.

From then until now, large number of advances in the development of this methodology have occurred. That, combined with the improvement of the processors speed and memories of the current computers make possible the simulation of more complex and realistic problems.

During the last 30 years, several authors have published about this method, such as [Sanchez and McCormick, 1982], [Marchuk and Lebedev, 1986], [Lewis and Miller Jr, 1984] or [Azmy and Sartori, 2010].

Since the first works developed by Carlson and Lathrop, [Lathrop and Carlson, 1964] or [Lathrop, 1968], great number of computer codes were developed based on $S_{N}$ method. The first code was DTF-IV, which was developed at Los Alamos National Laboratory by Lathrop [Lathrop, 1965], which uses diamond differences to solve one-dimensional transport. The next generation of codes developed by the same author were TWOTRAN and THREETRAN [Lathrop, 1976] that allows solving 2D and 3D problems respectively. In the eighties decade, an iteration acceleration method appears, the Diffusion Synthetic Acceleration (DSA). In general terms, as mentioned in [Azmy and Sartori, 2010], it consists in using Diffusion 
as an approximation to transport, with the purpose of calculating the iterative errors after source iteration. A complete review of the acceleration techniques is shown in [Adams and Larsen, 2002]. So, a new set of codes successors of the mentioned before emerged, ONEDANT, TWODANT and THREEDANT, [Alcouffe, 1994]. Later, Oak Ridge National Laboratory following the steps of the Los Alamos National Laboratory developed ANISN, DORT and TORT [Rhoades and Simpson, 1997]. More recently, with the continuous improvement of the computing and parallelization technologies, other parallelized codes have appeared like PARTISN [Alcouffe et al., 2005], DRAGON [Marleau et al., 2008], ARES [Chen et al., 2017], DENOVO [Evans et al., 2010], ATTILA [Youssef et al., 2013] and TITAN [Yi, 2009].

The main idea of this methodology is to discretize the angular variable in a set of discrete ordinates. It means that instead of considering that the particles can travel in any arbitrary direction on the unit sphere $\hat{\Omega}$, neutrons can only follow some finite number of directions. These number of directions $M$ are normally defined by $\hat{\Omega}_{n}$, with $1 \leq n \leq M$. Moreover, different weights are associated with each discrete ordinate $\hat{\Omega}_{n}$, these weights are defined by $\omega_{n}$. These concrete directions and its associated weights are specified by so-called Angular Quadratures. With these considerations the eq.2.4 remains:

$$
\begin{gathered}
\hat{\Omega}_{n} \cdot \nabla \psi_{g, n}(\vec{r})+\Sigma_{t, g}(\vec{r}) \psi_{g, n}(\vec{r})= \\
\sum_{g^{\prime}=1}^{G} \sum_{m=1}^{M} \Sigma_{s, g^{\prime} \rightarrow g}\left(\vec{r}, \hat{\Omega}_{m} \rightarrow \hat{\Omega}_{n}\right) \psi_{g^{\prime}, m}(\vec{r}) \omega_{m} \\
\quad+\frac{\chi_{g}(\vec{r})}{4 \pi} \sum_{g^{\prime}=1}^{G} \sum_{m=1}^{M} \nu \Sigma_{f, g^{\prime}}(\vec{r}) \psi_{g^{\prime}, m}(\vec{r}) \omega_{m}+\frac{Q_{g}(\vec{r})}{4 \pi}
\end{gathered}
$$

A quadrature set that allows an accurate angular variable integration is one of the most important things in the $S_{N}$ method. A very important property of the angular quadratures is that they satisfy a complete symmetry conditions. In fact, two are the principles that guided the development of the quadrature sets: physical symmetry and the arrangement of discrete directions on latitudes on the unit sphere. The study of quadrature sets for the Neutron Transport Equations starts with Lathrop and Carlson works [Lathrop and Carlson, 1964] where they dealt with the desired properties of the quadrature set for the discrete ordinates method. They presented in [Lathrop and Carlson, 1964] some of the quadratures developed in this thesis. 
In general, any angular quadrature set needs to satisfy a fundamental principle. It is that the integral over the unit sphere of any $f(\hat{\Omega})$ can be expressed as:

$$
\int_{4 \pi} f(\hat{\Omega}) d \hat{\Omega}=\sum_{n=1}^{M} \omega_{n} f\left(\hat{\Omega}_{n}\right)
$$

However, the mathematical rules to design quadrature sets are not clearly established as mentioned in Koch works, [Koch and Becker, 2004], [Koch et al., 1995]. Even some authors developed quadratures taking into account only pure geometrical considerations instead of considering mathematical aspects, [Rukolaine and Yuferev, 2001], [Thurgood et al., 1995].

In any case, the four general principles to develop Discrete Ordinates Method quadratures are:

1. All discrete ordinates must be placed on the unit sphere.

2. The weight factors $\omega_{n}$ must be positive.

3. The number of neutrons must be preserved.

4. Any rotation of the arrangement of the discrete ordinates directions around the center of the unit sphere must not affect the quadrature.

The first three principles are easy considerations, however with respect to the fourth principle, it is very difficult to achieve it with a limited number of discrete ordinates.

Several quadrature schemes have been developed for the Discrete Ordinates Method and transport theory as it can be seen in [Lee et al., 2015], [Lathrop and Carlson, 1964], [Carlson, 1970].

The most common quadrature sets are the Level Symmetric quadrature scheme $L S$ or $L Q_{N}$, which was developed by Lathrop and Carlson. However, they only can be used until order $S_{20}$. If a higher order is tried it gets negative weight values. In order to accomplish more moment conditions for integration accuracy, the Even-Odd moment quadratures set $E O_{N}$ were developed. They have the capability of integrating not only even moments but also odd moments. However they have also the limitation that the order can not be higher than $S_{16}$. Finally, The Equal Weight quadrature set $P_{N}$-EW solves the problem of the order limitation. One of the widely used quadrature is the half-symmetric Legendre-Chebyshev 
quadrature $P_{N}-T_{N}$ which does not present any limitation with the use of higher orders as well.

Furthermore, other quadrature sets beyond the classical quadrature sets have been developed by some authors, like in the $U E_{N}$ or $U G_{N}$ positive-weigth quadrature set [Carew and Zamonsky, 1999] or the $T_{N}$ quadrature set [Thurgood et al., 1995].

This thesis presents the formulation of the quadrature sets used that will be shown in next chapter. This allows the use of any order number without the use of tables.

\subsection{Diffusion Approximation}

Predicting the neutron distribution inside the system is essential in order to design nuclear cores correctly. However, to solve directly the neutron transport equation is not an easy task, since neutrons move following complicated paths after repeated arbitrary collisions. One simplification is to approximate the movement of the particles as a kind of diffusion as in gas theory occurs, when the trend of the particles is to pass from zones with more number of particles to others with less.

The neutron distribution considering this assumption can be calculated by means of solving the Diffusion equation. Most of the reactors constructed and operating today used this procedure for the major part of their calculations, which is normally called Diffusion Approximation.

The Diffusion approximation to the Boltzmann transport equation is commonly used in full-core simulations. The main reason of that is its relative simplicity and large range of applicability. Nevertheless, as mentioned in [Cacuci, 2010] "the diffusion approximation has several subtleties, and even today there are issues in its use that are not fully understood".

In any case, the neutron diffusion theory is considered the major building block for the design and operation of the nuclear reactors, [Lamarsh and Baratta, 2001]. Many authors have highlighted the importance of the Neutron Diffusion Equation for calculating the neutron distribution in full core simulations. The accuracy of this method is good enough to provide insight into the relevant physics in the nuclear reactors. 


\subsection{Simplified Spherical Harmonics}

As mentioned before, several variables forms the phase space for the solution of the transport equation. So, even today, solving three dimensional problems remains a difficult task for the current computers. Therefore, when Gelbard [Gelbard, 1960] started to develop the theory of the Simplified Spherical Harmonics $S P_{N}$ all eyes were on solving 3D Diffusion problems, and no one was thinking in transport problems far beyond academic purposes. However, the accuracy of the diffusion approximation is limited in some cases. In this gap between the Diffusion approximation and the Neutron Transport methods Gelbard introduces the $S P_{N}$ method [Gelbard, 1961].

The derivation of the $S P_{n}$ method arise from the idea of using the 1D slab geometry $P_{N}$ equations transformed somehow into 3D form. $P_{N}$ equations are the same than $S P_{N}$ for slab geometry. Generally, $S P_{N}$ provides better results compared with the diffusion approximation. During some time these equations were a little bit forgotten and the absence of theoretical basis did not help it even when the numerical results point in the right direction. That means that even though the justification of the method had not been derived, the improvement in the numerical solutions using these equations should have been considered.

Fortunately, several years before, other authors gave particular attention to the $S P_{N}$ method and tried to justify what had not been done. It was not until nineties when two independent papers came to light. In [Larsen et al., 1996] it was explained that the $S P_{N}$ were an asymptotic correction to standard diffusion theory while [Pomraning, 1993] demonstrated that $S P_{N}$ were asymptotically related to the $P_{N}$ for slab geometry.

However, the asymptotic derivations could generate several different equations equivalent to the $S P_{N}$ equations, so one could say that Gelbard guided these asymptotic derivations to a elegant form. Furthermore, the Pomraning work also used a variational analysis in which any $S P_{N}$ order could be derived. Other papers appeared later about material interfaces and boundary conditions, such as [Tomašević and Larsen, 1996] and [Brantley and Larsen, 2000]. In the last paper it was derived also the Marshak boundary conditions for the $S P_{N}$ equations.

Several works have been focused on $S P_{N}$ since then, like [Beckert and Grundmann, 2008], [Kotiluoto et al., 2007], [Hébert, 2010], [Vidal-Ferràndiz et al., 2020], new forms of those equations have been developed in [Chao, 2016] and a very efficient solution is shown in [Hamilton and Evans, 2015]. Moreover, a very interesting overview is presented in [McClarren, 2010]. 
Despite the fact that, nowadays there is a theoretical basis for the $S P_{N}$ equations, it should be said that they are not accurate for solving any kind of transport problems. Cases in which the problem is not locally one-dimensional or closely diffusive, the $S P_{N}$ results could be even worse than diffusion. A simple explanation of that is that $S P_{N}$ is an asymptotic approximation to the transport equation and away from the asymptotic limit there is no guarantee that the $S P_{N}$ method is good enough. Contrarily, in certain cases $S P_{N}$ provides equivalent solution to $P_{N}$.

\subsection{Finite Difference method}

The last step to arrive a completely discretized equation is the spatial discretization. Many different spatial discretization methods have been developed, among them it can be found Finite Difference Methods (FDM), Finite Element Methods (FEM), Finite Volume Methods (FVM) and Nodal Collocation Methods (NCM).

The Finite Difference Method is the simplest approach method to discretize partial differential equations. It is normally applied to a regular grid providing a very efficient solution methods. However, the method can not be used or it is not normally used for irregular geometries.

Two classical approaches exist for applying FDM to the discrete ordinates method in two-dimensional problems: Diamond scheme and Central Difference scheme [Davis et al., 1967]. Both discretization techniques were implemented in the first discrete ordinates programs. Central difference scheme was used in the early version of the TXY01 program. This program was developed for the Westinghouse Electric Corporation at the Bettis Atomic Power Laboratory in Pittsburgh, Pennsylvania. This code could solve monoenergetic neutron transport equation in a rectangular region, only for source problems with isotropric scattering [Natelson, 1971], [Bennett, 1964]. Diamond difference scheme was implemented in the program DDF of Los Alamos National Laboratory, which was based on the Lathrop and Carlson works [Carlson, 1968].

However, these techniques were affected by some convergence problems under certain considerations and they could give negative fluxes in some cases. A new difference method appeared some years later based on central difference equations that solve the problems of the negative fluxes [Madsen, 1974], it was called weighted central difference method WCD. Although this method solves, for particular choices of weighting factors, the convergence problem and the problem with negative fluxes, WCD had some limitations with respect to the problem size. W.A. Rhodes also published another formulation of these WCD, [Rhoades and 
Engle Jr, 1977]. This WCD was implemented in DOT IV code, [Rhoades, 1977]. Y.Y.Azmy also implemented a kind of weighted diamond difference method form for nodal transport method, [Azmy, 1988]. An interesting review of the methods can be found in [Liu et al., 1996].

More recently, an interesting comparison study is presented in [Duo and Azmy, 2007]. DENOVO code from Oak Ridge National Laboratory, which forms part of the SCALE code system and was developed to substitute TORT [Rhoades and Simpson, 1997], makes use as well of different finite difference schemes [Evans et al., 2010].

Definitely, despite having difficulty to perform calculations with irregular geometries, FDM methods continue to be an important part of the codes used currently, such as DENOVO among others.

\subsection{Modal Method and Krylov Schur}

After discretization is applied to the Neutron Transport Equation, the typical result is a very large system of algebraic equations to be solved. The system is defined by the matrix $A$ which represents the linear Boltzmann operator, the flux unknowns are defined by $\psi$ and $B$ contains the sources.

$$
A \psi=B
$$

Solving this by direct methods is complicated, since it is not practical to directly invert matrix $A$. Therefore, it is necessary to find more efficient strategies to solve the problem, and these strategies are based on iterative methods. One of the best strategies developed is the use of Krylov subspace methods, which are widely used.

During last decades, Krylov methods have presented an important influence in the way of how the $S_{N}$ equations have been solved. It was at the end of eighties beginning of nineties when the mathematicians applied successfully the Krylov methods to solve the $S_{N}$ equations, though it was not until last several years when Krylov methods have been widely used.

One of the advantages of the Krylov methods is the capability of calculating several eigenvalues. In particular the system of equations mentioned before can be defined as an eigenvalue problem as in eq.2.9, in which A and B are square matrices, $x$ is the eigenvector and $\lambda$ is the eigenvalue. When matrix B is not singular the 
problem can be simplified as in eq.2.10, in which $\mathcal{A}=B^{-1} A$. The eigenvalues of the eq.2.10 can be calculated by finding the roots of the characteristic polynomial or by diagonalizing $\mathcal{A}$. Nevertheless, an important computational cost is required to process these operations for large matrices. But the sparse conditions of these matrices and the fact that only few eigenvalues are needed, make possible the use of other type of methods. These are the iterative methods, which allows solving efficiently these kind of problems.

$$
\begin{gathered}
A x=\lambda B x \\
\mathcal{A} x=\lambda x
\end{gathered}
$$

There are several iterative methods that allows to solve eigenvalue problems:

- Single vector iteration

- Subspace iteration

- Krylov subspace

- Davidson methods

- Newton methods

- Block Newton methods

The first methods used with sparse matrices are the single vector iteration. These methods are based on the principle that a vector that is repeatedly multiplied by matrix $\mathcal{A}$ normally tends to align in the direction of the eigenvector associated to its dominant eigenvalue. The most commonly methods with this principle are the Power iteration method, Inverse iteration and Rayleigh quotient iteration. One of the disadvantages of this method is that they are not competitive calculating several eigenvalues.

In this regard, the Subspace iteration and the Krylov subspace methods present some advantages. These are called projection methods and an interesting overview can be found in [Saad and Schultz, 1986]. In general terms, it can be said that Subspace iteration method is inferior to Krylov methods. Examples of Krylov methods are Lanczos, Arnoldi and Krylov-Schur, being the last two commented in following lines. This research work is focused in these methods because they are commonly used to obtain the eigenvalues in the spectrum extremes of standard eigenvalue problems [Bernal García, 2018]. 
Other method mentioned is the Davidson method which is used to calculate interior eigenvalues. However, in Reactor Physics, the important eigenvalues are located in the extremes. On the other hand, Newton methods are fast in particular for non-linear problems and generalized eigenvalue problems. Finally, Block Newton methods have the same principles than the Newton methods but they are indicated to calculate more efficiently multiple eigenvalues.

As mentioned before, this thesis uses the Krylov-Shur method, which is based on the Arnoldi method. The Arnoldi method computes an orthonormal basis of the Krylov subspace of order $m$ associated with matrix $\mathcal{A}$ and vector $x_{0}$. Eq.2.11 defines the Krylov subspace.

$$
\mathcal{K}_{m}\left(\mathcal{A}, x_{0}\right)=\operatorname{span}\left\{x_{0}, \mathcal{A} x_{0}, \mathcal{A}^{2} x_{0}, \ldots, \mathcal{A}^{m-1} x_{0}\right\}
$$

The main intent of the projection methods is to compute a partial eigensolution. This means that given a matrix $\mathcal{A}$ of order $N$, the aim is to compute a small number of eigenpairs, $\lambda_{i}, x_{i}$ with $i=1, \ldots, m$ and $m \ll N$. The Arnolid method simultaneously computes the orthonormal basis $V_{m}$ and the projection of this basis in the matrix $A$, which is the upper Hessenberg matrix $H$. So, the Arnoldi method instead of calculating the eigenvalue problem $\mathcal{A} x_{i}=\lambda_{i} x_{i}$ of order $N$, it calculates the eigenvalue problem $H y_{i}=\Theta_{i} y_{i}$ of order $m$. In other words, considering $H=V_{m}^{T} \mathcal{A} V_{m}$ and $V_{m}^{T} V_{m}=I_{m}$, it can be said that the pair $\left(\lambda_{i}, V_{m} y_{i}\right)$ are approximated to the eigenpair $\left(\lambda_{i}, x_{i}\right)$ of matrix $\mathcal{A}$.

The convergence of this method is extremely fast when the initial vector $x_{0}$ is close to the solution. Unfortunately, it is not normally the case and several iteration are required.

Ultimately, Krylov-Schur methods, which are a type of Arnoldi methods, make use of a Krylov-Schur decomposition instead of a Krylov decomposition. That means that a real Schur form of $\mathcal{A}$ can be obtained, rather than the upper Hessenberg matrix. Some publications in this regard are [Stewart, 2002], [Hernández et al., 2007], [Bernal et al., 2017], [Vidal-Ferrandiz et al., 2014], [Carreño et al., 2017] and [Bernal et al., 2018b].

The solution of linear systems is essential for solving eigenvalue problems. In particular, linear systems are involved in the calculation of the product of the inverse matrix and a vector. Since high computational costs are needed to calculate the inverse of a matrix, it is better to solve the following linear system:

$$
x=\mathcal{A}^{-1} b \rightarrow \mathcal{A} x=b
$$


The best methods, for solving the previous linear systems when the matrices are not too large are those called Direct Methods, such as, Gaussian elimination, Cramer's rule or LU factorization. They calculate in an exact way the linear system. However these methods are not efficient with large matrices.

Iterative methods are best suited for large matrices, however the efficiency of these methods depend on the well conditioning of the matrix. If the condition number of the matrices is good, iterative methods require less computational resources than direct solvers. Contrarily, if the matrices are ill-conditioned, the convergence of the iterative method cannot be achieved and it is necessary to use of direct methods.

The condition number reflects the inaccuracy in calculating the solution $x$ once the numerical method has been applied. The condition number can be calculated as the ratio between the largest singular value and the smallest one, as shown in eq.2.13. This ratio is a property of the matrix and independent of the numerical method used to solve the system.

$$
\mathcal{C}=\frac{\sigma_{\text {largest }}(\mathcal{A})}{\sigma_{\text {smallest }}(\mathcal{A})}
$$

Another important aspect of the iterative methods is that they require preconditioners in order to accelerate the convergence of the method. The preconditioner is a matrix multiplying the linear system. The definition of the iterative methods and preconditioners is beyond the scope of this thesis, but most commonly used iterative methods and preconditioners are listed in table 2.1.

Table 2.1: Iterative methods and preconditioners

\begin{tabular}{c|c}
\hline Iterative methods & Preconditioners \\
\hline Bi-Conjugate Gradient (BiCG) & Jacobi \\
Conjugate Gradients Squared (CGS) & Incomplete LU \\
BiCGSTAB (Bi-Conjugate Gradient stabilized) & SOR \\
Generalized Minimal Residual (GMRES) & Additive Schwarz \\
Generalized Conjugate Residual (GCR) & \\
\hline
\end{tabular}

There exist several libraries and software with specialized algorithms to solve the methods mentioned before for solving eigenvalue problems and linear systems. One of the most commonly used is SLEPc. SLEPc library called Scalable Library for Eigenvalue Problem Computations (SLEPc) is a software library for the solution of large, sparse eigenproblems on parallel computers [Hernandez et al., 
2005]. SLEPc is the state of the art for calculating eigenproblems of large and sparse matrices like those obtained with the methods of this thesis. In addition, SLEPc uses PETSc [Balay et al., 2019] (Portable, Extensible Toolkit for Scientific Computation) to extend it with all the necessary functionalities to obtain the solution of eigenvalue problems; which includes matrix operations and the solution of linear systems. SLEPc and PETSc are widely used to solve eigenvalue problems and linear system respectively. Furthermore, MUMPS (MUltifrontal Massively Parallel sparse direct Solver) is a library which provides better direct solvers for linear systems [Amestoy et al., 1998]. 



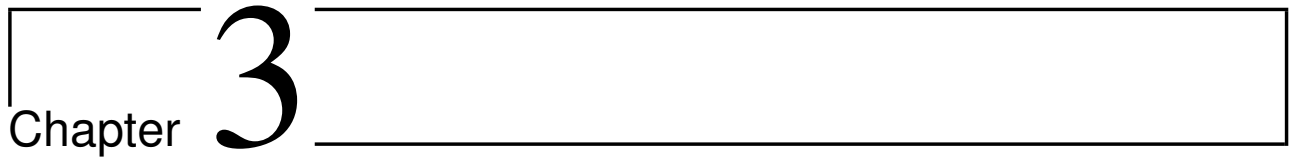

\section{Neutron Transport Equation}

This chapter is devoted to the derivation of the Neutron Transport Equation. A general description of the variables which define the Neutron Transport Equation is carried out, as well as, the definitions in the fixed source, eigenvalues and explicitly time-independent forms that are points of departure for numerical computation. The chapter explains the formulation of the neutron transport equation used in this thesis. These formulations are based on the Finite Difference Method and the Discrete Ordinates Method.

\subsection{Background for Transport Theory}

Neutron Transport is the process in which neutrons propagate through the atoms in a physical system. This migration involves a large number of random events. This includes different interactions such as the scattering of neutrons, capture of neutrons and the initiation by neutrons of fission events.

An important issue for designing and analyzing nuclear reactors is the accurate prediction of the position, angle, energy and time dependence of neutrons inside all the components of the nuclear system.

The transport of non-charged particles such as neutrons and photons, is accurately described by the Transport Equation, which is called the linear Boltzmann equation. By solving this equation the particle distribution is obtained. 
Analytical solutions of the Neutron Transport Equation can be calculated only for simple problems that are not realistic. Currently, for realistic problems with multidimensional geometries and heterogeneous materials, the Neutron Transport Equation can only be solved by using numerical methods.

For steady-state systems, the neutron distribution depends on 6 variables: three spatial variables, two angular variables and one energy variable. Numerical methods applied to solve the Neutron Transport Equation can be classified into 2 groups, stochastic (Monte Carlo) or deterministic methods.

Monte Carlo methods are very different from deterministic methods. Monte Carlo does not solve an explicit equation. It provides the solution by means of simulate individual particles and the average behavior of these simulating particles gives an estimate of average quantities of interest in the system.

By contrast, Deterministic methods solve an integro-differential transport equation by determining the six independent variables for the average particle behavior. The energy variable is commonly discretized using the multigroup approximation. The spatial variables can be discretized with several methods, such as Finite Volume Method (FVM), Finite Element Method (FEM), Nodal Expansion Method (NEM) or the Finite Difference Method (FDM). To discretize the angular variable, the most commonly used methods are, the Spherical Harmonics $\left(P_{N}\right)$ or the Discrete Ordinates $\left(S_{N}\right)$. This thesis is focused on the Deterministic method Discrete Ordinates and Finite Difference method.

\subsubsection{Physical Variables of the Neutron Transport Equation}

The neutron transport equation is an integro-differential equation with seven independent variables (only six considering a steady-state system). This set of variables shown in fig. 3.1 sometimes is called phase space and it is composed of:

- 3 components of the position vector $\vec{r}=(x, y, z)=x \mathbf{i}+y \mathbf{j}+z \mathbf{k}$ (Cartesian Coordinates)

- 2 angles to specify the unit vector $\hat{\Omega}$ denoting the direction of the neutron: $(\theta, \omega) \rightarrow(\mu, \omega)$ where $\mu=\cos (\theta)$

- Kinetic Energy $\boldsymbol{E}$

- Time $\boldsymbol{t}$ 


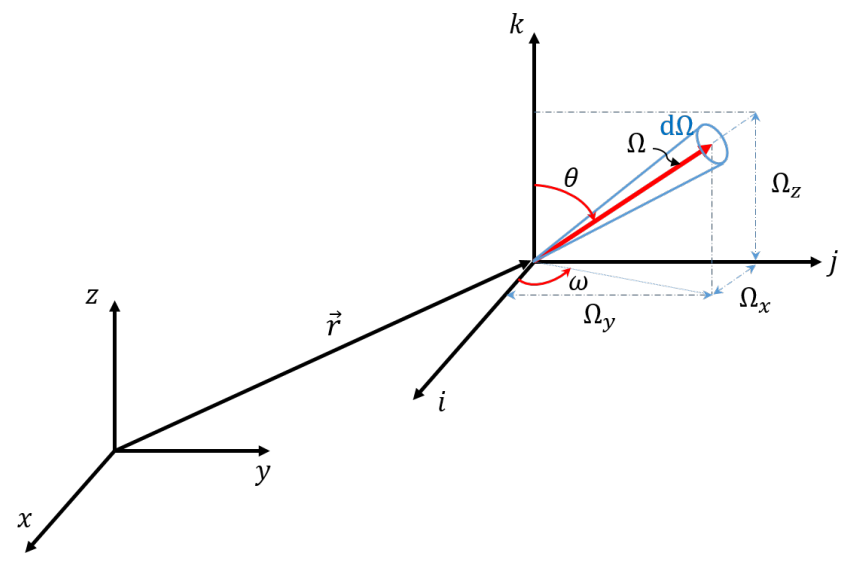

Figure 3.1: The spatial $(\vec{r})$, angular $(\hat{\Omega})$, and energy $(E)$ variables.

The polar angle $\theta$ defined relative to $z$-axis and the azimuthal angle relative to $x$-axis on the $X Y$-plane characterize the direction vector $\hat{\Omega}$ which is a unit vector $|\hat{\Omega}|=1$.

$$
\hat{\Omega}=f(\theta, \omega)=\Omega_{x} i+\Omega_{y} j+\Omega_{z} k=(\eta, \xi, \mu)
$$

\subsubsection{Cross Section definition}

As [Cacuci, 2010] and [Lewis and Miller Jr, 1984] explain, considering the next incrementally thin neutron target, when the neutron travels an incremental distance $d s$, exist an incremental probability $d p$ that the neutron could interact with a nucleus.


Figure 3.2: Thin neutron target. 
If it is considered that the neutron is normally incident, at an arbitrary point, on the target area $A$ and incremental thickness $d s$, one can determine the relationship between $d p$ and $d s$. Assuming that the microscopic cross-section area of a target nucleus $\sigma(E)\left(\mathrm{cm}^{2}\right)$ and the number density of target nuclei $N\left(\mathrm{~cm}^{3}\right)$ are known. If the target is thin enough and each target nucleus does not shield another, in that case the probability of a collision is:

$$
\begin{gathered}
d p=\frac{\text { total area of nuclei }}{\text { area of target }}=\frac{n \sigma}{A} \\
n=N d V=N(A \cdot d s)=\text { number of nuclei in target } \\
d p=\frac{N(A \cdot d s) \sigma}{A}=(N \sigma) d s
\end{gathered}
$$

Hence, $d p$ is proportional to $d s$

$$
\begin{gathered}
d p=\Sigma(E) d s \\
\Sigma(E)=N \sigma(E)=\text { macroscopic cross section }\left(\mathrm{cm}^{-1}\right)
\end{gathered}
$$

So, the total macroscopic cross section can be defined as:

$$
\begin{gathered}
\Sigma_{t}(E)=\sum_{i} \Sigma_{i}(E)=\Sigma_{\text {capture }}(E)+\Sigma_{\text {scatter }}(E)+\Sigma_{\text {fission }}(E) \\
\Sigma_{i}(E)=\Sigma_{t}(E) \cdot p_{i}(E) \quad \sum_{i} p_{i}(E)=1
\end{gathered}
$$




\section{Scattering Particle Distribution}

Before defining the transport equation, it is necessary to specify the probability distribution laws that govern the emission of scattered particles in energy and angle. When the neutron is captured, it is considered to be removed from the system, conversely, if the neutron with direction $\hat{\Omega}$ and energy $E$ scatters, it emerges after the scattering event with new direction $\hat{\Omega}^{\prime}$ and new energy $E^{\prime}$ :

$$
p\left(\hat{\Omega} \cdot \hat{\Omega}^{\prime}, E \rightarrow E^{\prime}\right) d \hat{\Omega}^{\prime} d E^{\prime}
$$

This is the probability that the scattered neutron from energy $E$ and direction $\hat{\Omega}$, will emerge in an energy interval $d E^{\prime}$ about $E^{\prime}$ traveling in a solid angle $d \hat{\Omega}^{\prime}$ about $\hat{\Omega}^{\prime}$. The scattering in media with randomly distributed scattering centers (nuclei) is rotationally invariant. That is, the probability that a neutron will scatter from direction $\hat{\Omega}$ to direction $\hat{\Omega}^{\prime}$ depends only on the scattering angle $\theta_{0}$ between $\hat{\Omega}$ and $\hat{\Omega}^{\prime}$ (or, on the cosine of this angle, $\mu_{0}=\cos \left(\theta_{0}\right)=\hat{\Omega} \cdot \hat{\Omega}^{\prime}$ ).

This is a consequence of the assumption that the medium is isotropic, so that, there is no preference toward any particular values of the azimuthal angle, $\omega$. For brevity of notation the incremental solid angle $\hat{\Omega}$ is defined as:

$$
\begin{aligned}
& d \hat{\Omega}=\frac{d \omega}{2 \pi} \frac{d \theta \sin \theta}{2}=\frac{d \omega}{2 \pi} \frac{d \mu}{2} \\
& \int d \hat{\Omega}=\int_{0}^{2 \pi} \frac{d \omega}{2 \pi} \int_{-1}^{1} \frac{d \mu}{2}=1
\end{aligned}
$$

Then, if a scattering collision is considered, in which one particle is emitted:

$$
\int d E^{\prime} \int d \hat{\Omega}^{\prime} f\left(E \rightarrow E^{\prime}, \hat{\Omega} \cdot \hat{\Omega}^{\prime}\right)=1
$$

Thus, all scattering directions $\hat{\Omega}^{\prime}$ on the cone of equal scattering angle are equally probable. The distribution function for elastic s-wave neutron scattering, which is isotropic in the center of mass frame, can be shown from kinematics [Duderstadt and Hamilton, 1976b] to be given by: 


$$
p\left(\hat{\Omega} \cdot \hat{\Omega}^{\prime}, E \rightarrow E^{\prime}\right)=p_{0}\left(E \rightarrow E^{\prime}\right) \cdot \frac{\delta\left[\hat{\Omega} \cdot \hat{\Omega}^{\prime}-\mu_{0}\left(E \rightarrow E^{\prime}\right)\right]}{2 \pi}
$$

The term $p_{0}\left(E \rightarrow E^{\prime}\right)$ is a histogram distribution function for outgoing energies $E^{\prime}$ :

$$
p_{0}\left(E \rightarrow E^{\prime}\right)= \begin{cases}\frac{1}{(1-\alpha) E} & \text { if } \quad \alpha E<E^{\prime}<E \\ 0 & \text { otherwise }\end{cases}
$$

Where: $\alpha=\left(\frac{A-1}{A+1}\right)^{2}$ and $\mathrm{A}$ is the nucleus-to-neutron mass ratio. Also, $\delta$ is the common delta function. Thus, the outgoing neutron energy $E^{\prime}$ is random and uniformly distributed between $\alpha E$ and $E$. Once, $E^{\prime}$ is known, the scattering cosine is uniquely specified by:

$$
\mu_{0}\left(E \rightarrow E^{\prime}\right)=\left(\frac{A+1}{2}\right) \sqrt{\frac{E^{\prime}}{E}}-\left(\frac{A-1}{2}\right) \sqrt{\frac{E}{E^{\prime}}}
$$

However, the scattering azimhutal angle $\omega^{\prime}$ is random and uniformly distributed on $0 \leq \omega^{\prime} \leq 2 \pi$. The minimum neutron energy loss associated with the minimal change in direction of the neutron ("forward" scattering, $\mu_{s}=+1$ ), while the maximum neutron energy loss is associated with the maximum change of the neutron ("backward" scattering, $\mu_{s}=-1$ ). The macroscopic differential scattering cross section can be defined with dimension $\mathrm{cm}^{-1} \mathrm{MeV}^{-1}$ as:

$$
\Sigma_{s}\left(\hat{\Omega} \cdot \hat{\Omega}^{\prime}, E \rightarrow E^{\prime}\right)=\Sigma_{s}(E) p\left(\hat{\Omega} \cdot \hat{\Omega}^{\prime}, E \rightarrow E^{\prime}\right)
$$

The probability that a neutron, with direction $\hat{\Omega}$ and energy $E$ and traveling a distance (unit path length), will scatter into a solid angle $d \hat{\Omega}^{\prime}$ about $\hat{\Omega}^{\prime}$ and into an energy interval $d E^{\prime}$ about $E^{\prime}$ is:

$$
\Sigma_{s}\left(\hat{\Omega} \cdot \hat{\Omega}^{\prime}, E \rightarrow E^{\prime}\right) d s d \hat{\Omega}^{\prime} d E^{\prime}
$$



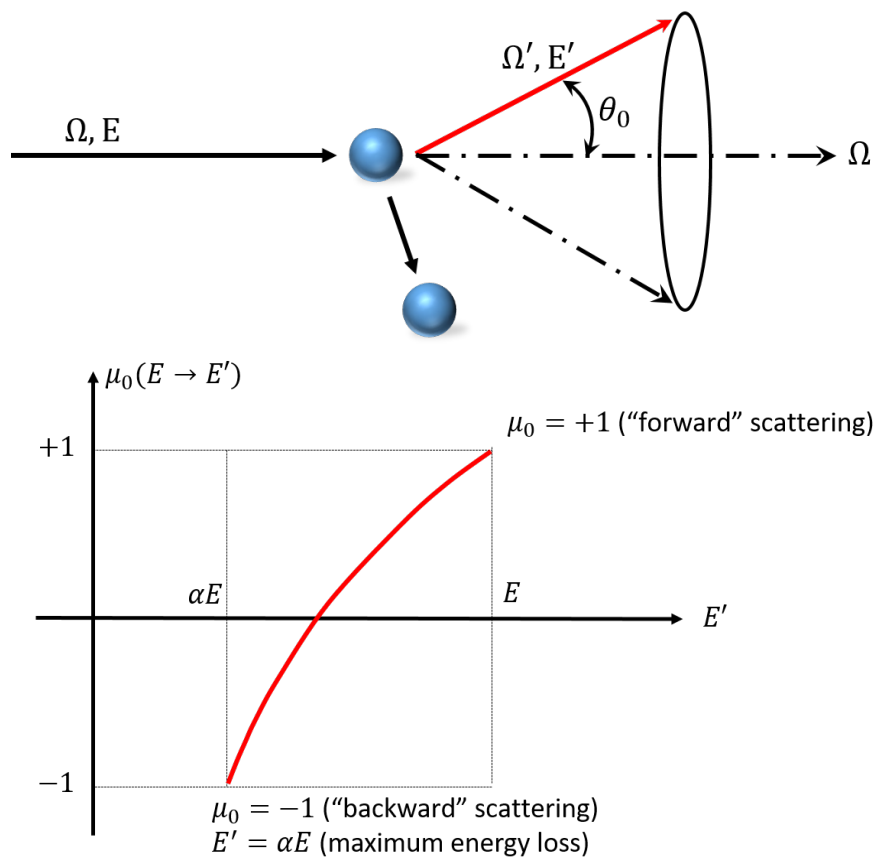

Figure 3.3: Neutron probability.

For most deterministic transport methods, it is common to expand differential scattering cross sections into orthogonal Legendre polynomials $P_{l}\left(\mu_{0}\right)$, where $\mu_{0}=$ $\Omega \cdot \hat{\Omega}^{\prime}$ is the cosine of the scattering angle, as will be explained later.

$$
\begin{gathered}
\int_{0}^{\infty} \int_{4 \pi} p\left(\hat{\Omega} \cdot \hat{\Omega}^{\prime}, E \rightarrow E^{\prime}\right) d \hat{\Omega}^{\prime} d E^{\prime}=1 \\
\int_{0}^{\infty} \int_{4 \pi} \Sigma_{s}\left(\hat{\Omega} \cdot \hat{\Omega}^{\prime}, E \rightarrow E^{\prime}\right) d \hat{\Omega}^{\prime} d E^{\prime}=\sigma_{s}(E)
\end{gathered}
$$




\section{Fission Neutron Distribution}

Fission neutrons can be considered that they are produced isotropically. There are some differences between fission and scattering reactions. The energy of the fission spectrum is independent of the energy of the neutron causing the fission and secondary neutrons are produced by fission event. Two quantities must be described to define the reaction by which a neutron of energy $E$ produces a fission neutron of energy $E^{\prime}$ :

- $\nu(E)=$ the mean number of fission neutrons produced in a fission caused by a neutron with energy $E$.

- $\chi_{p}\left(E^{\prime}\right) d E^{\prime}=$ the probability that a fission neutron will have an energy within $d E^{\prime}$ about $E^{\prime}$.

From [Lewis and Miller Jr, 1984], for a particular fissionable isotope, $\nu_{i}(E)$ tends to be nearly constant, increasing monotonically with energy by 10 percent or less over the entire energy range of interest.

When a neutron with energy $E$ initiates a fission event, the target nucleus is divided into two smaller daughter nuclei releasing on the average, $\nu(E)$ neutrons. From this number of released neutrons, $\nu(E)[1-\beta(E)]$ are prompt which means that they are emitted within $10^{-15 s}$ of the fission event and $\nu(E) \beta(E)$ are delayed, emitted approximately $0.1-60 s$ after fission event. So, the probability that a fission neutron, created by a neutron with energy $E$, is delayed $(\beta \approx 0.01)$ is the delayed neutron fraction $\beta(E)$. Delayed fission neutrons are created from the radioactive decay of unstable daughter nuclei, which can be produced during fission events [Cacuci, 2010].

Prompt fission neutrons appear at the location of the fission event, their initial direction of flight is isotropic, i.e. uniformly distributed on the unit sphere, and their initial energy is consistent with the prompt fission spectrum $\chi_{p}\left(E^{\prime}\right)$. This definition of $\chi_{p}\left(E^{\prime}\right)$ satisfies:

$$
\int_{0}^{\infty} \chi_{p}\left(E^{\prime}\right) d E^{\prime}=1
$$






Figure 3.4: Typical fission spectrum plotted as a function of the neutron energy.

Six groups are normally considered to group the unstable daughter nuclei, each with its own radioactive decay constant $\lambda_{j}$, delayed fraction $\beta_{j}(E)$, and delayed neutron fission spectrum $\chi_{j}\left(E^{\prime}\right)$, where $1 \leq j \leq 6$. The functions $\beta_{j}(E)$ obey:

$$
\sum_{j=1}^{6} \beta_{j}(E)=\beta(E)
$$

Finally, one can define the probable number of fission neutrons produced at $\vec{r}$ with energies within $d E^{\prime}$ about $E^{\prime}$ within the cone of angles $d \hat{\Omega}$ about $\hat{\Omega}$ per path length traveled by neutrons with energy $E$, where $\Sigma_{f}$ is the macroscopic fission cross section.

$$
\nu(E) \cdot \Sigma_{f}(r, E) \cdot \chi_{p}\left(E^{\prime}\right) d E^{\prime} d \hat{\Omega}
$$

When more than one fissionable isotope is involved this quantity becomes:

$$
\left.\chi\left(E^{\prime}\right) \cdot \nu_{(} E\right) \cdot \Sigma_{f}(r, E)=\chi_{p}\left(E^{\prime}\right) \sum_{i} \nu_{i}(E) \cdot \Sigma_{f}^{i}(r, E)
$$




\subsubsection{Magnitudes in Neutron Transport}

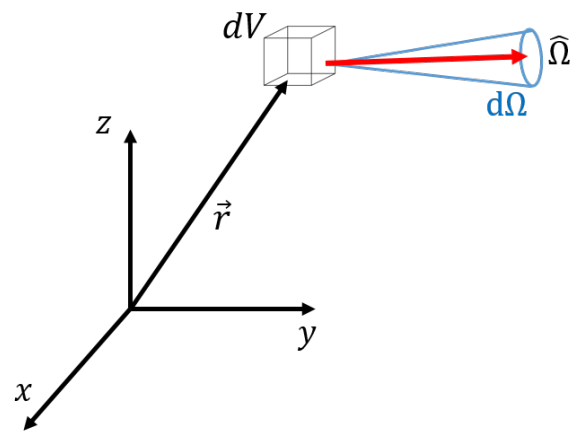

Figure 3.5: $d V$ about $\vec{r}, d \hat{\Omega}$ about $\hat{\Omega}$.

Considering particles in an incremental volume $d V$ about $\vec{r}$, which travel in cone of directions $d \hat{\Omega}$ about direction $\hat{\Omega}$ as seen in Fig.3.5. Normalizing the incremental angle $d \hat{\Omega}$ by Eqs.3.9 and 3.10 one could define the particle density distribution in the six-dimensional phase space defined by $\vec{r}, \hat{\Omega}, E$ and at time $t$ to obtain Eq.3.23, the expected number of neutrons in a volume element $d V$ about $\vec{r}$ traveling in the cone of directions $d \hat{\Omega}$ about $\hat{\Omega}$ with energies between $E$ and $E+d E$ at time $t$ [Lewis and Miller Jr, 1984].

$$
N(\vec{r}, \hat{\Omega}, E, t) d V d E d \hat{\Omega}
$$

Some other dependent variables convenient to use can be expressed in terms of the density distribution. So, this is a fundamental dependent variable. Many times, it is more natural to formulate transport problems using the angular flux or fluence rate, instead of density distribution. The angular flux can be defined by:

$$
\psi(\vec{r}, \hat{\Omega}, E, t) \equiv \nu \cdot N(\vec{r}, \hat{\Omega}, E, t)
$$

where $\nu=\sqrt{2 E / m}$ is the neutron speed.

There are two useful physical interpretations from [Cacuci, 2010] for $\psi$. One is the total of the path lengths traveled by neutrons, in $d V d \hat{\Omega} d E$ about $(\vec{r}, \hat{\Omega}, E)$, 
during time increment $d t$ about $t$, being the distance that the particle travels $\nu d t$ during $d t$.

$$
\psi(\vec{r}, \hat{\Omega}, E, t) d V d \hat{\Omega} d E d t=\nu \cdot N(\vec{r}, \hat{\Omega}, E, t) d V d \hat{\Omega} d E d t
$$

The second one is the volume-based interpretation, it is the rate at which path length is generated by neutrons in $d V d \hat{\Omega} d E$ about $(\vec{r}, \hat{\Omega}, E)$ at time $t$ :

$$
\psi(\vec{r}, \hat{\Omega}, E, t) d V d \hat{\Omega} d E
$$

Considering the macroscopic cross section for the $i$ interaction type $\Sigma_{i}(\vec{r}, E)$, the total number of reactions per unit time in $d V d E d \hat{\Omega}$ is:

$$
\Sigma_{i}(\vec{r}, E) \cdot \psi(\vec{r}, \hat{\Omega}, E, t) d V d E d \hat{\Omega}
$$

Some occasions, the direction that the particles are traveling is irrelevant in calculating reaction rates. Therefore, scalar flux is defined as the integral of $\psi$ over all directions:

$$
\phi(\vec{r}, E, t)=\int \psi(\vec{r}, \hat{\Omega}, E, t) d \hat{\Omega}
$$

So, it is simple to define the number of reactions in $d V d E$ per unit time as:

$$
\Sigma_{i}(\vec{r}, E) \cdot \phi(\vec{r}, E, t) d V d E
$$

When the incremental volume in phase space is dropped, then $\Sigma_{i} \cdot \phi$ is referred as reaction rate or if $\Sigma_{i}$ is the total cross section, as the collision density [Lewis and Miller Jr, 1984].

In order to explain another common use of the angular flux, suppose we have an incremental surface $d A$, where the normal to the surface is $\vec{n}$ as shown in Fig.3.6. 

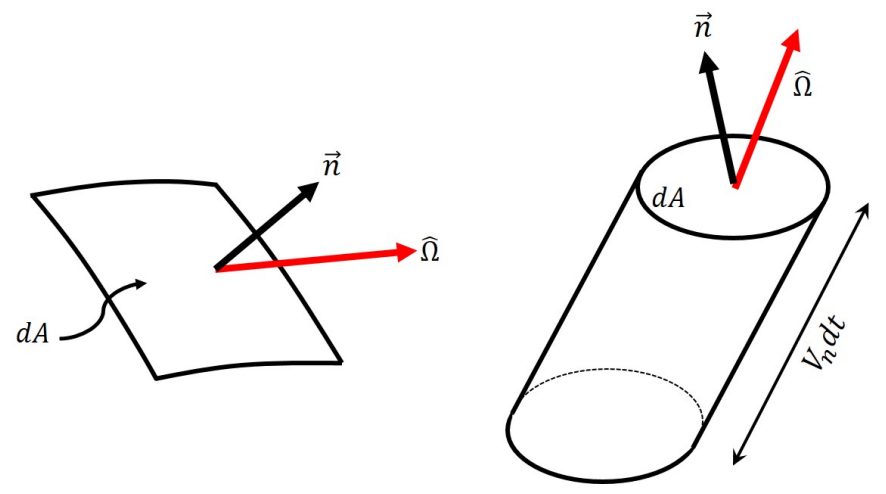

Figure 3.6: Neutron flow through $d A$ and representation of the volume $\nu d t n \hat{\Omega} d A$.

In order to determine the number of particles passing through $d A$, with energies between $E$ and $E+d E$ that are going in particular direction $\hat{\Omega}$ during the time from $t$ to $t+d t$. This quantity is just the number of particles in the volume defined by $\nu \cdot d t \cdot \vec{n} \cdot \hat{\Omega} \cdot d A$ :

$$
N(\vec{r}, \hat{\Omega}, E, t) \nu d t \vec{n} \cdot \hat{\Omega} d A d E=\vec{n} \cdot \hat{\Omega} \psi(\vec{r}, \hat{\Omega}, E, t) d A d t d E
$$

The net number of particles with energy between $E$ and $E+d E$ crossing $d A$ in the direction of $\vec{n}$ regardless of $\hat{\Omega}$ during $d t$ is:

$$
\int \hat{\Omega} \vec{n} \cdot \hat{\Omega} \psi(\vec{r}, \hat{\Omega}, E, t) d A d t d E
$$

The current vector is expressed as:

$$
\vec{J}(\vec{r}, E, t) \equiv \int d \hat{\Omega} \hat{\Omega} \psi(\vec{r}, \hat{\Omega}, E, t)
$$

For a given particle energy $E$ and given time $t$, the dot product of the eq.3.33 gives the net number of particles crossing per unit area of surface, per unit time and energy in the positive $\vec{n}$ direction.

$$
J_{n}(\vec{r}, E, t) \equiv \vec{J}(\vec{r}, E, t)=\int d \hat{\Omega} \vec{n} \cdot \hat{\Omega} \psi(\vec{r}, \hat{\Omega}, E, t)
$$


The current can be calculated from the incoming and outcoming currents, as shown in eq.3.34:

$$
J_{n}(\vec{r}, E, t)=J_{n}^{+}(\vec{r}, E, t)-J_{n}^{-}(\vec{r}, E, t)
$$

where

$$
\begin{aligned}
J_{n}^{+}(\vec{r}, E, t) & =\int_{\hat{\Omega} \vec{n}>0} d \hat{\Omega} \vec{n} \cdot \hat{\Omega} \psi(\vec{r}, \hat{\Omega}, E, t) \\
J_{n}^{-}(\vec{r}, E, t) & =\int_{\hat{\Omega} \vec{n}<0} d \hat{\Omega}|\vec{n} \cdot \hat{\Omega}| \psi(\vec{r}, \hat{\Omega}, E, t)
\end{aligned}
$$
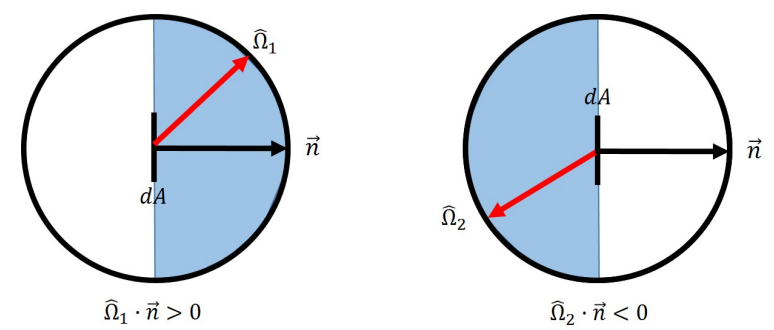

Figure 3.7: Positive and negative directions of the neutron flow through $d A$.

\subsection{The time dependent Neutron Transport Equation}

This chapter is devoted to formulating the neutron transport equation and describing each term of this equation, which is just a neutron balance inside a control volume. In addition, the neutron transport problems can be classified by how is their source of neutrons, this source can be fixed, e.g. when the source is an encapsulate radionuclide or an X-Ray generator or distributed like occurs in a nuclear reactor.

As seen in previous chapter, one needs to consider the following phase space in the unit sphere, where $\theta$ is the polar angle (relative to the z-axis), $\omega$ is the azimuthal angle (relative to the $\mathrm{x}$-axis on the XY-plane), $\hat{\Omega}$ is the direction, $d \hat{\Omega}$ is the cone of directions and $E$ is the energy of the neutrons. 


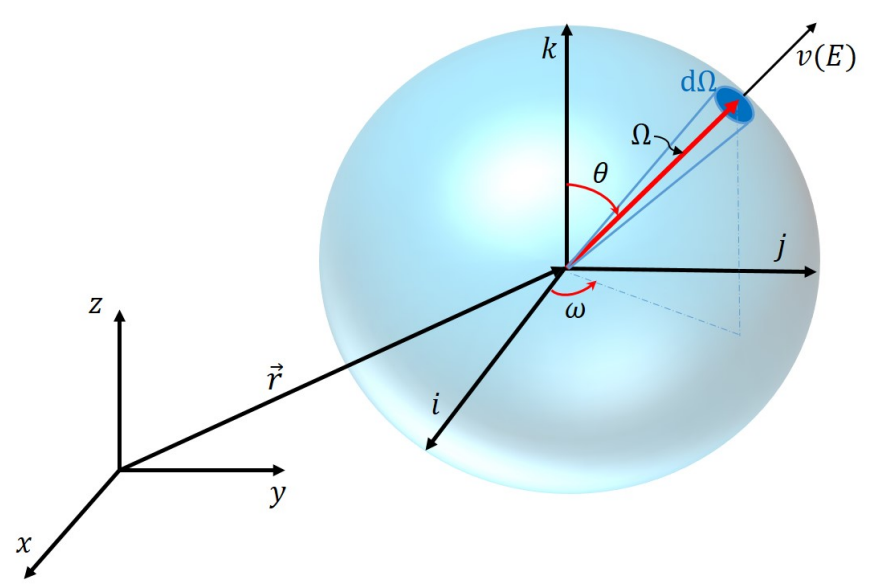

Figure 3.8: Neutron's phase space in the unit sphere.

As defined previously, $\vec{r}=(x, y, z)$ and $\hat{\Omega}=f(\theta, \omega)=\Omega_{x} i+\Omega_{y} j+\Omega_{z} k=$ $(\eta, \xi, \mu)$, then the number of neutrons in the control volume $V$ in the position $\vec{r}$ with energy $E$ in the time $t$ and direction $\hat{\Omega}$ is:

$$
\int_{V} N(\vec{r}, \hat{\Omega}, E, t) d V d E d \hat{\Omega}
$$

Deriving, the time rate change of the number of neutrons can be obtained:

$$
\frac{\partial}{\partial t} \int_{V} N(\vec{r}, \hat{\Omega}, E, t) d V=\text { gain in } V-\text { loss in } V
$$

If the control volume is independent on time, considering $\psi(\vec{r}, \hat{\Omega}, E, t)=\nu$. $N(\vec{r}, \hat{\Omega}, E, t)$ the following expression can be defined:

$$
\frac{\partial}{\partial t} \int_{V} N(\vec{r}, \hat{\Omega}, E, t) d V=\int_{V} \frac{N(\vec{r}, \hat{\Omega}, E, t) d V}{\partial t}=\frac{1}{\nu} \int_{V} \frac{\psi(\vec{r}, \hat{\Omega}, E, t) d V}{\partial t}
$$

Taking into account the mechanisms described in table.3.1, the transport equation can be expressed as a balance equation 3.40: 
Table 3.1: Gain and loss mechanisms

\begin{tabular}{l|l}
\hline GAIN MECHANISMS & LOSS MECHANISMS \\
\hline $\begin{array}{l}\text { 1. Any neutron source in } V \text { (fission, } \\
\text { or fixed source) }\end{array}$ & $\begin{array}{l}\text { 4. Neutrons leaking out through the } \\
\text { surface } d A\end{array}$ \\
$\begin{array}{l}\text { 2. Neutrons streaming into } V \\
\text { through the surface } d A\end{array}$ & $\begin{array}{l}\text {. Neutrons in } V \text { undergoing a col- } \\
\text { lision (for example absorption and } \\
\text { scattering out } E, \hat{\Omega})\end{array}$ \\
$\begin{array}{l}\text { 3. Neutrons of different } E^{\prime}, \hat{\Omega}^{\prime} \text { suffer- } \\
\text { ing a scattering collision in } V \text { change } \\
\text { into } E, \hat{\Omega} \text { of interest }\end{array}$ & \\
\hline
\end{tabular}

$$
\left[\frac{1}{\nu} \int_{V} \frac{\partial \psi(\vec{r}, \hat{\Omega}, E, t) d V}{\partial t}\right] d \hat{\Omega} d E=(1)+(2)+(3)-(4)-(5)
$$

The source term (1) represents any neutron creation inside the volume coming from a fixed source (independent source) or fission reactions (dependent source since it depends on the number of neutrons interacting within the control volume).

$$
(1)=\left[\int_{V} Q(\vec{r}, \hat{\Omega}, E, t) d V\right] d \hat{\Omega} d E
$$

where $Q(\vec{r}, \hat{\Omega}, E, t) d V$ is the rate of source neutrons appearing in $d V$ about $\vec{r}$, with energy $E$ and direction $\hat{\Omega}$.

The incoming streaming term takes into account the neutrons that enter inside the control volume through its surface, and can be expressed as:

$$
J_{n}^{-}(\vec{r}, E, t)=\int_{0}^{\infty} \int_{S} \int_{\hat{\Omega} \vec{n}<0}|\vec{n} \cdot \hat{\Omega}| \psi(\vec{r}, \hat{\Omega}, E, t) d \hat{\Omega} d S d E
$$

The outgoing leaking term takes into account the neutrons that exit from out control volume through its surface to the exterior, and can be expressed according to Eq.3.35:

$$
J_{n}^{+}(\vec{r}, E, t)=\int_{0}^{\infty} \int_{S} \int_{\hat{\Omega} \vec{n}>0} \vec{n} \cdot \hat{\Omega} \psi(\vec{r}, \hat{\Omega}, E, t) d \hat{\Omega} d S d E
$$


The last two terms often are very difficult to understand separately. For that, to understand better these terms we are going to consider a sphere, with two differentiated surfaces, which is reached by neutrons in the direction $\hat{\Omega}$.

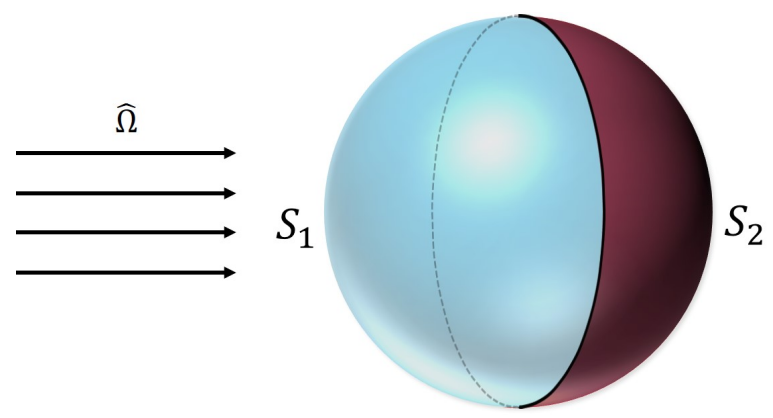

Figure 3.9: Sphere reached by neutrons with direction $\hat{\Omega}$.

When the neutrons reached the surface (S1), we are considering it as streaming in. Taking into account the sign criterion of the current vector (Eq.3.34) this is a negative value due to the dot product $\hat{\Omega} \cdot \vec{r}<0$, but this have no sense considering the sign of the Eq.3.38 where every neutron entering in our control volume is positive. On the other hand, if neutron reached the surface (S2) with the same direction $\hat{\Omega}$, they are streaming out from our control volume; however this current vector is positive.

We solve this question combining these terms in one that provide us the net number of particles streaming out of the control volume. As in Eq.3.34, if one considers leaking-out term minus streaming-in term, one obtains the net rate at which neutrons flow (leak) out of the control volume at time $t$ :

$$
\begin{array}{r}
(4)-(2)=J_{n}^{+}(\vec{r}, E, t)-J_{n}^{-}(\vec{r}, E, t)= \\
\int_{S} \int_{\hat{\Omega} \vec{n}>0} \vec{n} \cdot \hat{\Omega} \psi(\vec{r}, \hat{\Omega}, E, t) d \hat{\Omega} d S d E- \\
\int_{S} \int_{\hat{\Omega} \vec{n}<0}|\vec{n} \cdot \hat{\Omega}| \psi(\vec{r}, \hat{\Omega}, E, t) d \hat{\Omega} d S d E= \\
\int_{S} \int_{4 \pi}(\vec{n} \cdot \hat{\Omega}) \psi(\vec{r}, \hat{\Omega}, E, t) d \hat{\Omega} d S d E
\end{array}
$$


Can be seen that the last equation is developed in terms of element $d S$. However, most cases it is interesting to convert the equation in terms of a volume element $d V$. Using the divergence theorem one can achieve:

$$
\begin{gathered}
\int_{S} d S \vec{n} \cdot f(r)=\int_{V} d V \nabla \cdot f(r) \\
(4)-(2)=\left[\int_{S}(\vec{n} \cdot \hat{\Omega}) \psi(\vec{r}, \hat{\Omega}, E, t) d S\right] d \hat{\Omega} d E= \\
{\left[\int_{V}(\hat{\Omega} \cdot \nabla) \psi(\vec{r}, \hat{\Omega}, E, t) d V\right] d \hat{\Omega} d E}
\end{gathered}
$$

Then, Eq.3.46 is the net rate at which neutron flux leak out of the control volume at time $t$, and we will consider it in the Eq. 3.38 as a negative term, because it is a loss of neutrons.

The gain neutron streaming into $d E$ about $E, d \hat{\Omega}$ about $\hat{\Omega}$ from other energies and directions can be defined as:

$$
(3)=\left[\int_{V} \int_{4 \pi} \int_{0}^{\infty} \Sigma_{s}\left(\vec{r}, E^{\prime} \rightarrow E, \hat{\Omega}^{\prime} \rightarrow \hat{\Omega}\right) \psi\left(\vec{r}, E^{\prime}, \hat{\Omega}^{\prime}, t\right) d E^{\prime} d \hat{\Omega}^{\prime} d V\right] d \hat{\Omega} d E
$$

The rate at which neutrons suffer collisions at point $r$ is:

$$
f_{t}=\Sigma_{t}(r, E) \psi(\vec{r}, \hat{\Omega}, E, t)
$$

$\Sigma_{t}$ is the macroscopic total cross section defined in Eq.3.6 Hence, integrating this collision rate over the volume, one finds:

$$
(5)=\left[\int_{V} \Sigma_{t}(r, E) \psi(\vec{r}, E, \hat{\Omega}, t) d V\right] d \hat{\Omega} d E
$$

Substituting each term in the Eq.3.40 one has: 


$$
\begin{array}{r}
{\left[\frac{1}{\nu} \int_{V} \frac{\partial \psi(\vec{r}, \hat{\Omega}, E, t) d V}{\partial t}\right] d \hat{\Omega} d E=(1)+(3)-(4-2)-(5)=} \\
{\left[\int_{V} Q(\vec{r}, \hat{\Omega}, E, t) d V\right] d \hat{\Omega} d E} \\
+\left[\int_{V} \int_{4 \pi} \int_{0}^{\infty} \Sigma_{s}\left(\vec{r}, E^{\prime} \rightarrow E, \hat{\Omega}^{\prime} \rightarrow \hat{\Omega}\right) \psi\left(\vec{r}, E^{\prime}, \hat{\Omega}^{\prime}, t\right) d E^{\prime} d \hat{\Omega}^{\prime} d V\right] d \hat{\Omega} d E \\
-\left[\int_{V}(\hat{\Omega} \cdot \nabla) \psi(\vec{r}, \hat{\Omega}, E, t) d V\right] d \hat{\Omega} d E \\
-\left[\int_{V} \Sigma_{t}(r, E) \psi(\vec{r}, E, \hat{\Omega}, t) d V\right] d \hat{\Omega} d E
\end{array}
$$

Rearranging terms:

$$
\begin{array}{r}
d V\left[\frac{1}{\nu} \int_{V} \frac{\partial \psi(\vec{r}, \hat{\Omega}, E, t)}{\partial t}+\int_{V}(\hat{\Omega} \cdot \nabla) \psi(\vec{r}, \hat{\Omega}, E, t)+\int_{V} \Sigma_{t}(r, E) \psi(\vec{r}, E, \hat{\Omega}, t)\right. \\
-\int_{V} Q(\vec{r}, \hat{\Omega}, E, t) \\
\left.\quad-\int_{V} \int_{4 \pi} \int_{0}^{\infty} \Sigma_{s}\left(\vec{r}, E^{\prime} \rightarrow E, \hat{\Omega}^{\prime} \rightarrow \hat{\Omega}\right) \psi\left(\vec{r}, E^{\prime}, \hat{\Omega}^{\prime}, t\right) d E^{\prime} d \hat{\Omega}^{\prime}\right] d \hat{\Omega} d E=0
\end{array}
$$

However, let me now apply the fact that the volume $\mathrm{V}$ was quite arbitrarily chosen. Hence, the only way for the integral to vanish for any $\mathrm{V}$ is for its integrand to be identically zero, that is:

$$
\int_{\text {any } V} d V f(r)=0 \rightarrow f(r)=0
$$

Hence, the balance relation can be expressed as: 


$$
\begin{array}{r}
\frac{1}{\nu} \frac{\partial \psi(\vec{r}, \hat{\Omega}, E, t)}{\partial t}+(\hat{\Omega} \cdot \nabla) \psi(\vec{r}, \hat{\Omega}, E, t)+\Sigma_{t}(r, E) \psi(\vec{r}, E, \hat{\Omega}, t) \\
=\int_{4 \pi} \int_{0}^{\infty} \Sigma_{s}\left(\vec{r}, E^{\prime} \rightarrow E, \hat{\Omega}^{\prime} \rightarrow \hat{\Omega}\right) \psi\left(\vec{r}, E^{\prime}, \hat{\Omega}^{\prime}, t\right) d E^{\prime} d \hat{\Omega}^{\prime}+Q(\vec{r}, \hat{\Omega}, E, t)=0
\end{array}
$$

It occasionally proves convenient to restrict out study to isotropic scattering processes and source distributions for which $f\left(\hat{\Omega}^{\prime} \rightarrow \hat{\Omega}\right)=\frac{1}{4 \pi}$ and $Q(\vec{r}, \hat{\Omega}, E)=$ $\frac{Q(\vec{r}, E)}{4 \pi}$. Under these assumptions the steady state transport equation simplifies still further to:

$$
\begin{aligned}
& {\left[\hat{\Omega} \cdot \nabla+\Sigma_{t}(r, E)\right] \psi(\vec{r}, \hat{\Omega}, E)} \\
& =\frac{1}{4 \pi} \int_{4 \pi} \int_{0}^{\infty} \Sigma_{s}\left(\vec{r}, E^{\prime} \rightarrow E, \hat{\Omega}^{\prime} \rightarrow \hat{\Omega}\right) \psi\left(\vec{r}, E^{\prime}, \hat{\Omega}^{\prime}\right) d E^{\prime} d \hat{\Omega}^{\prime}+\frac{Q(\vec{r}, E)}{4 \pi}
\end{aligned}
$$

In fission chain reacting systems, the source contribution from fission neutrons is explicitly extracted and written as:

$$
\frac{Q(\vec{r}, E, \hat{\Omega})}{4 \pi}=Q_{f}(\vec{r}, E, \hat{\Omega})=\frac{\chi(E)}{4 \pi} \int_{0}^{\infty} d E^{\prime} \int_{4 \pi} d \hat{\Omega}^{\prime} \nu\left(E^{\prime}\right) \Sigma_{f}\left(r, E^{\prime}\right) \psi\left(\vec{r}, E, \hat{\Omega}^{\prime}\right)
$$

where $\nu\left(E^{\prime}\right)$ is the average number of neutrons released per fission event, and $\chi(E)$ is the energy distribution or spectrum of the fission neutrons.

\subsection{D steady state Neutron Transport Equation with Isotropic Scattering}

This chapter provides a simple derivation of the one-dimensional Neutron Transport Equation with isotropic scattering. This simple equation will serve as a start point from which more complicated derivations introducing 2 dimensional problems and anisotropic scattering will be performed.

To simplify, different energies are not considered, so the Neutron Transport Equation (Eq.3.54) could be wrote as: 


$$
\left[\hat{\Omega} \cdot \nabla+\Sigma_{t}(r)\right] \cdot \psi(\vec{r}, \hat{\Omega})=\frac{1}{4 \pi} \int_{4 \pi} \Sigma_{s}\left(r, \hat{\Omega}^{\prime} \rightarrow \hat{\Omega}\right) \psi\left(\vec{r}, \hat{\Omega}^{\prime}\right) d \hat{\Omega}^{\prime}+\frac{Q(\vec{r})}{4 \pi}
$$

Considering plane symmetry $\left(\Omega_{x} \equiv \mu=\cos \theta ; \psi=\psi\left(x, \Omega_{x}\right)=\psi(x, \mu) ; \Omega\right.$. $\left.\nabla \psi(x, \mu)=\mu \frac{\partial \psi}{\partial x}\right)$.

$$
\mu \frac{\partial \psi(x, \mu)}{\partial x}+\Sigma_{t}(x) \psi(x, \mu)=\frac{1}{2} \Sigma_{s}(x) \int_{-1}^{+1} d \mu^{\prime} \psi\left(x, \mu^{\prime}\right)+Q(x)
$$

\subsubsection{Discrete Ordinates Method}

The Discrete Ordinates method consists in considering only a set of directions $\mu_{n}$ and apply a quadrature approximation to the integral term. To solve the equation, one can define $N$ discrete directions $\left(\mu_{1}, \mu_{2}, \ldots, \mu_{N}\right)$ with $-1 \leq \mu \leq+1$ and corresponding weighting coefficients $\left(w_{1}, w_{2}, \ldots, w_{N}\right)$. A review of this method was published by [Lathrop and Carlson, 1964]. In Discrete Ordinates equations the scalar flux is approximated by the following quadrature formula:

$$
\phi(x)=\int_{-1}^{1} \psi\left(x, \mu^{\prime}\right) d \mu^{\prime} \approx \frac{1}{2} \sum_{m=1}^{N} w_{m} \psi\left(x, \mu_{m}\right)
$$

Note that the flux approximation is divided by 2 , that is because the quadrature formula is normalized by

$$
\sum_{m=1}^{N} w_{m}=2 \quad \text { with } \quad w_{m}>0
$$

Owing to this discretization of the angular coordinate, the eq.3.55 is transformed into eq.3.60:

$$
\mu_{n} \frac{\partial \psi\left(x, \mu_{n}\right)}{\partial x}+\Sigma_{t}(x) \psi\left(x, \mu_{n}\right)=\frac{1}{2} \Sigma_{s}(x) \sum_{m=1}^{N} \omega_{m} \psi\left(x, \mu_{m}\right)+Q\left(x, \mu_{n}\right)
$$

where: 


$$
Q\left(x, \mu_{n}\right)=\frac{1}{2} \nu \Sigma_{f}(x) \int_{-1}^{+1} d \mu^{\prime} \psi\left(x, \mu^{\prime}\right) \approx \frac{1}{2} \nu \Sigma_{f}(x) \sum_{m=1}^{N} \omega_{m} \psi\left(x, \mu_{m}\right)
$$

\subsubsection{Fixed source and eigenvalue problem}

A critical system in the nuclear field is one which contains fissionable material and it is able to allow self-sustaining time-independent chain reaction in the absence of an external neutron source. That means, if neutrons are inserted in a critical system, after sufficient time when the transient effects disappear, then, will exist a time independent asymptotic neutron distribution in which the rate of fission neutron production is just equal to the absorption and leakage terms.

If this equilibrium is not reachable, the asymptotic distribution of neutrons (called the fundamental mode) will not be steady state and will either increase or decrease with time exponentially as indicated in Fig.3.10a. Then, the system can be said supercritical or subcritical, respectively. If a time independent external neutron source is considered in a subcritical system, it tends an equilibrium state characterized by a time independent neutron flux distribution in which the production rate of external and fission neutrons is in equilibrium with the absorption and leakage terms. Conversely, if the system is critical or supercritical, the equilibrium is impossible in the presence of an external source, and the neutron flux distribution will be an increasing function of time. This is shown in Fig.3.10b.

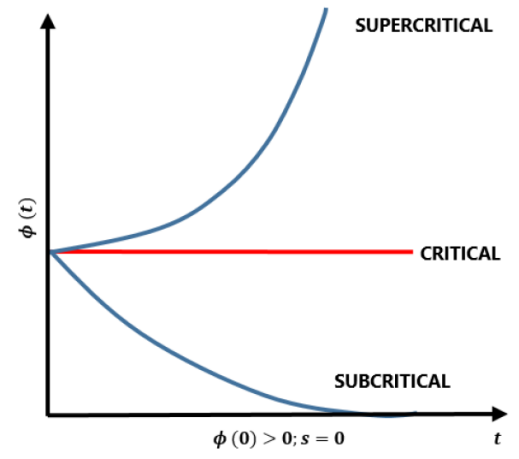

(a)

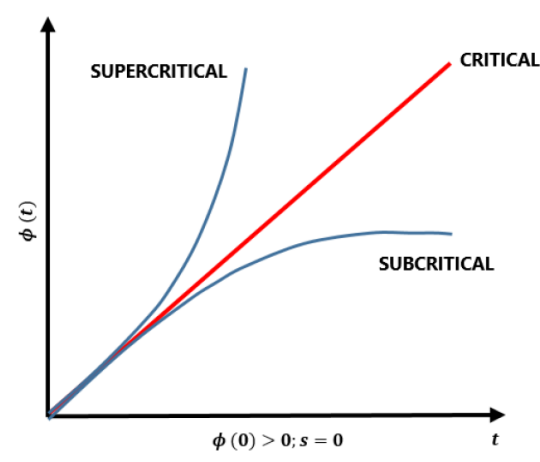

(b)

Figure 3.10: (3.10a) Time dependence of the flux for a source-free multiplying medium. (3.10b) Time dependence of the flux for a multiplying medium with a known source. 
Assuming that $\nu$, the average number of neutrons per fission, can be adjusted to obtain a time-independent solution to Eq.3.59 and 3.60, the $k$ eigenvalue problem or eigenvalue form of the criticality problem can be formulated as in Eq.3.62, where $\nu$ is substituted by $\nu / k$ :

$$
\begin{array}{r}
\mu_{n} \frac{\partial \psi\left(x, \mu_{n}\right)}{\partial x}+\Sigma_{t}(x) \psi\left(x, \mu_{n}\right) \\
=\frac{1}{2} \Sigma_{s}(x) \sum_{m=1}^{N} \omega_{m} \psi\left(x, \mu_{m}\right)+\frac{1}{2 k} \nu \Sigma_{f}(x) \sum_{m=1}^{N} \omega_{m} \psi\left(x, \mu_{m}\right)
\end{array}
$$

In matrix form:

$$
A \cdot \psi=\frac{1}{k} B \cdot \psi
$$

Clearly the system is critical if $k=1$. A value of $k<1$ implies that the hypothetical number of neutrons per fission, $\nu / k$, required to make the system just critical is largest than $\nu$, the number available in reality. Hence the system is subcritical. Conversely, for $k>1$, fewer neutrons per fission are required to make the system critical than are produced in reality, and thus the system is supercritical. To summarize,

$$
k \begin{cases}>1 & \text { supercritical } \\ =1 & \text { critical } \\ <1 & \text { subcritical }\end{cases}
$$

\subsubsection{Finite Difference Method for 1D}

The spatial variables are discretized with the Finite Difference Method. First, one defines a one-dimensional spatial grid with $I$ mesh points. The cross-sections are taken to be constant inside each interval $\left(x_{i-1 / 2}, x_{i+1 / 2}\right)$. Moreover, the cellcentered points are defined by:

$$
x_{i}=\frac{1}{2}\left(x_{i-1 / 2}+x_{i+1 / 2}\right)
$$

and defining: 


$$
\Sigma(x)=\Sigma(i) \quad \text { with } \quad x_{i-1 / 2}<x<x_{i+1 / 2}
$$

The flux derivative term and the flux are approximated by:

$$
\begin{gathered}
\frac{\partial}{\partial x} \psi\left(x, \mu_{n}\right)=\frac{\psi\left(i+1 / 2, \mu_{n}\right)-\psi\left(i-1 / 2, \mu_{n}\right)}{h} \\
\psi\left(x, \mu_{n}\right)=\frac{\psi\left(i+1 / 2, \mu_{n}\right)+\psi\left(i-1 / 2, \mu_{n}\right)}{2}
\end{gathered}
$$

where $h=x_{i+1 / 2}-x_{i-1 / 2}=\Delta x_{i}$.

Therefore, the one-dimensional Neutron Transport Equation with Discrete Ordinates method and Finite Difference Method can be written as:

$$
\begin{array}{r}
\mu_{n} \frac{\psi\left(i+1 / 2, \mu_{n}\right)-\psi\left(i-1 / 2, \mu_{n}\right)}{h}+\Sigma_{t}(i) \frac{\psi\left(i+1 / 2, \mu_{n}\right)+\psi\left(i-1 / 2, \mu_{n}\right)}{2} \\
=\frac{1}{2} \Sigma_{s}(i) \sum_{m=1}^{N} \omega_{m} \frac{\psi\left(i+1 / 2, \mu_{m}\right)+\psi\left(i-1 / 2, \mu_{m}\right)}{2} \\
+\frac{\chi(i)}{2 k} \nu \Sigma_{f}(i) \sum_{m=1}^{N} \omega_{m} \frac{\psi\left(i+1 / 2, \mu_{m}\right)+\psi\left(i-1 / 2, \mu_{m}\right)}{2}
\end{array}
$$

\subsubsection{Boundary Conditions for 1D Discrete Ordinates Method}

The boundary conditions commonly used in neutron transport problems are vacuum, periodic, reflective and albedo conditions. One of the advantages of considering an even ordinate set for one-dimensional cases is that the vacuum, reflective or albedo boundary conditions are simply approximated by

$$
\begin{aligned}
& \psi_{n}(i)=\beta \cdot \psi_{N+1-n}(i) \quad \text { with } \quad i=0 \text { and } n=1,2, \ldots, N / 2 \\
& \text { or } \\
& \\
& \quad i=I \text { and } n=\frac{N}{2}+1, \frac{N}{2}+2, \ldots, N
\end{aligned}
$$


where if:

$\beta=0$ (vacuum condition) $; \beta=1$ (reflective condition) $; \beta \in] 0,1[$ (albedo condition)

The periodic boundary condition can be defined by Eq.3.71.

$$
\psi_{0}^{n}=\psi_{I}^{n} \quad \text { with } 1 \leq n \leq N
$$

\subsection{D steady state Neutron Transport Equation with Anisotropic Scattering}

The one-dimensional steady-state Neutron Transport Equation [Lewis and Miller Jr, 1984] can be expressed as :

$$
\begin{gathered}
\mu \frac{\partial \psi(x, \mu, E)}{\partial x}+\Sigma_{t}(x, E) \psi(x, \mu, E)=\int_{0}^{\infty} \int_{-1}^{1} \Sigma_{s}\left(x, \mu^{\prime}, \mu, E^{\prime} \rightarrow E\right) \psi\left(x, \mu^{\prime}, E^{\prime}\right) d \mu^{\prime} d E^{\prime} \\
+\frac{1}{k_{e f f}} \chi(x, E) \int_{0}^{\infty} \int_{-1}^{1} \nu\left(x, E^{\prime}\right) \Sigma_{f}\left(x, E^{\prime}\right) \psi\left(x, \mu^{\prime}, E^{\prime}\right) d \mu^{\prime} d E^{\prime}
\end{gathered}
$$

Where:

$$
\begin{aligned}
x & : \text { Spatial variable } \\
\mu & : \text { Angular variable or director cosine } \\
E & : \text { Energy } \\
\psi(x, \mu, E) & : \text { Angular neutron flux } \\
\Sigma_{t}(x, E) & : \text { Macroscopic total cross-section } \\
\Sigma_{s}\left(x, \mu^{\prime}, \mu, E^{\prime} \rightarrow E\right) & : \text { Macroscopic scattering cross-section } \\
& \text { from energy } E^{\prime} \text { to } E \text { and from direction } \mu^{\prime} \text { to } \mu \\
\chi(x, E) & : \text { Fission spectrum } \\
\nu\left(x, E^{\prime}\right) & : \text { Average number of neutrons generated per fission } \\
\Sigma_{f}\left(x, E^{\prime}\right) & : \text { Macroscopic fission cross-section } \\
k_{e f f} & : \text { Multiplication Factor }
\end{aligned}
$$


To derive the multi-group equations, one divides the neutron energy range into $G$ intervals which are the energy groups. The particles in group $g$ are those with energies between $E_{g}$ and $E_{g-1}$, hence the group number increases as the energy decreases. Then the angular flux for group $g$ can be expressed as (see fig.2.1):

$$
\psi_{g}(x, \mu)=\int_{E_{g}}^{E_{g-1}} \psi(x, \mu, E) d E
$$

Moreover, if one divides the energy integrals in Eq.3.72, into the contributions for each energy group:

$$
\int_{0}^{\infty} \psi\left(x, \mu, E^{\prime}\right) d E^{\prime}=\sum_{g^{\prime}=1}^{G} \int_{E_{g^{\prime}}}^{E_{g^{\prime}-1}} \psi\left(x, \mu, E^{\prime}\right) d E^{\prime}
$$

For brevity the shorthand notation of the Eq.3.73 is employed:

$$
\int_{g} d E=\int_{E_{g}}^{E_{g-1}} d E
$$

and integrating 3.72 between $E_{g}$ and $E_{g-1}$ one obtains:

$$
\begin{gathered}
\mu \frac{\partial}{\partial x} \int_{g} \psi(x, \mu, E) d E+\int_{g} \Sigma_{t}(x, E) \psi(x, \mu, E) d E \\
=\sum_{g^{\prime}=1}^{G} \int_{g} \int_{g^{\prime}} \int_{-1}^{1} \Sigma_{s}\left(x, \mu^{\prime}, \mu, E^{\prime} \rightarrow E\right) \psi\left(x, \mu^{\prime}, E^{\prime}\right) d \mu^{\prime} d E^{\prime} d E \\
+\frac{1}{k_{e f f}} \int_{g} \chi(x, E) \sum_{g^{\prime}=1}^{G} \int_{g^{\prime}} \int_{-1}^{1} \nu\left(x, E^{\prime}\right) \Sigma_{f}\left(x, E^{\prime}\right) \psi\left(x, \mu^{\prime}, E^{\prime}\right) d \mu^{\prime} d E^{\prime} d E
\end{gathered}
$$

Assuming that within each energy group the angular flux can be approximated as the product of a known function of energy $f(E)$ and the group flux $\psi_{g}(x, \mu)$, the multigroup cross sections can be defined as:

$$
\Sigma_{t, g}(x)=\int_{g} \Sigma_{t}(x, E) f(E) d E
$$




$$
\begin{gathered}
\nu \Sigma_{f, g}(x)=\int_{g} \nu(x, E) \Sigma_{f}(x, E) f(E) d E \\
\Sigma_{s, g^{\prime} \rightarrow g}\left(x, \mu^{\prime}, \mu\right)=\int_{g} \int_{g^{\prime}} \Sigma_{s}\left(x, \mu^{\prime}, \mu, E^{\prime} \rightarrow E\right) f\left(E^{\prime}\right) d E^{\prime} d E
\end{gathered}
$$

and let:

$$
\chi_{g}(x)=\int_{g} \chi(x, E) d E
$$

and considering:

$$
\int_{g} f(E) d E=1
$$

then one could write Eq.3.76 in the conventional multi-group form:

$$
\begin{aligned}
\mu \frac{\partial}{\partial x} \psi_{g}(x, \mu)+\Sigma_{t, g}(x) \psi_{g}(x, \mu) & \\
& =\sum_{g^{\prime}=1}^{G} \int_{-1}^{1} \Sigma_{s, g^{\prime} \rightarrow g}\left(x, \mu^{\prime}, \mu\right) \psi_{g^{\prime}}\left(x, \mu^{\prime}\right) d \mu^{\prime} \\
& +\frac{1}{k_{e f f}} \chi_{g}(x) \sum_{g^{\prime}=1}^{G} \int_{-1}^{1} \nu \Sigma_{f, g^{\prime}}(x) \psi_{g^{\prime}}\left(x, \mu^{\prime}\right) d \mu^{\prime}
\end{aligned}
$$

On the other hand, the anisotropic scattering cross section is commonly written as a Legendre Polynomial Expansion and the angular flux can be expanded as a Legendre series of degree L. [Lewis and Miller Jr, 1984]. 


$$
\begin{aligned}
& \int_{-1}^{1} \Sigma_{s, g^{\prime} \rightarrow g}\left(x, \mu^{\prime}, \mu\right) \psi_{g}\left(x, \mu^{\prime}\right) d \mu^{\prime} \\
& \quad=\int_{-1}^{1} \sum_{k=0}^{\infty}(2 k+1) \Sigma_{s, g^{\prime} \rightarrow g, k}(x) P_{k}(\mu) P_{k}\left(\mu^{\prime}\right) \sum_{l=0}^{L}(2 l+1) P_{l}\left(\mu^{\prime}\right) \phi_{g^{\prime}, l}(x) d \mu^{\prime}
\end{aligned}
$$

Considering the orthogonality relation of the Legendre Polynomials:

$$
\int_{-1}^{1} P_{k}(\mu) P_{l}(\mu) d \mu=\frac{1}{2 l+1} \delta_{k l}
$$

where $\delta_{k l}$ is the Kronecker delta, which is equal to 1 if $k=l$ and 0 otherwise. Eq.3.83 takes the form:

$$
\int_{-1}^{1} \Sigma_{s, g^{\prime} \rightarrow g}\left(x, \mu^{\prime}, \mu\right) \psi_{g}\left(x, \mu^{\prime}\right) d \mu^{\prime}=\sum_{l=0}^{L}(2 l+1) P_{l}(\mu) \Sigma_{s, g^{\prime} \rightarrow g, l}(x) \phi_{g^{\prime}, l}(x)
$$

The Legendre moments $\Sigma_{s, g^{\prime} \rightarrow g, l}$ are calculated for each material region [Azmy and Sartori, 2010]. The Discrete Ordinates method consists in considering only a set of directions $\mu_{n}$ and apply a quadrature approximation to the integral term. To solve the equation, one can define $N$ discrete directions $\left(\mu_{1}, \mu_{2}, \ldots, \mu_{N}\right)$ with $-1 \leq \mu \leq+1$ and corresponding weighting coefficients $\left(w_{1}, w_{2}, \ldots, w_{N}\right)$. In Discrete Ordinates equations the scalar flux is approximated by the following quadrature formula:

$$
\phi(x)=\int_{-1}^{1} \psi(x, \mu) d \mu=\frac{1}{2} \sum_{n=1}^{N} w_{n} \psi\left(x, \mu_{n}\right)
$$

and the Legendre moments by:

$$
\phi_{l}(x)=\frac{1}{2} \sum_{n=1}^{N} w_{n} P_{l}\left(\mu_{n}\right) \psi\left(x, \mu_{n}\right)
$$

In eq.3.86 and eq.3.87 the flux and the Legendre moment approximation are divided by 2 because the quadrature formula is normalized by 


$$
\sum_{n=1}^{N} w_{n}=2 \quad \text { with } \quad w_{n}>0
$$

By including Eqs.3.85-3.87 in Eq.3.82, the following equation is obtained:

$$
\begin{aligned}
& \mu \frac{\partial \psi_{g}(x, \mu)}{\partial x}+\Sigma_{t, g}(x) \psi_{g}(x, \mu) \\
& =\sum_{g^{\prime}=1}^{G} \sum_{l=0}^{L}(2 l+1) P_{l}(\mu) \Sigma_{s, g^{\prime} \rightarrow g, l}(x) \frac{1}{2} \sum_{n=1}^{N} w_{n} P_{l}\left(\mu_{n}\right) \psi_{g^{\prime}}\left(x, \mu_{n}\right) \\
& \quad+\frac{1}{K_{e f f}} \chi_{g}(x) \sum_{g^{\prime}=1}^{G} \nu \Sigma_{f, g^{\prime}}(x) \frac{1}{2} \sum_{n=1}^{N} w_{n} \psi\left(x, \mu_{n}\right)
\end{aligned}
$$

The choice of the weighting factors $w_{N}$ is commonly made with respect to an even number of discrete ordinates $\mu_{N}$ chosen in a symmetric way with respect to $\mu=0$. Hence, one can define this group of directions and corresponding weighting coefficients as:

$$
\begin{gathered}
\mu_{n}>0 \\
\mu_{N+1-n}=-\mu_{n} \quad \text { for } \quad n=1,2, \ldots, \frac{N}{2} . \\
w_{N+1-n}=w_{n}
\end{gathered}
$$

Finally, the Finite Difference Method is used for the spatial discretization. To discretize the spatial variable one define a one-dimensional spatial grid with $I$ mesh points. The cross-sections are taken to be constant inside each interval $\left(x_{i-1 / 2}, x_{i+1 / 2}\right)$. Moreover, the cell-centered points are defined by:

$$
x_{i}=\frac{1}{2}\left(x_{i-1 / 2}+x_{i+1 / 2}\right)
$$

and defining:

$$
\Sigma(x)=\Sigma(i) \quad \text { with } \quad x_{i-1 / 2}<x<x_{i+1 / 2}
$$

The flux derivative term and the flux are approximated by: 


$$
\begin{gathered}
\frac{\partial}{\partial x} \psi_{g}(x, \mu)=\frac{\psi_{g}\left(i+1 / 2, \mu_{n}\right)-\psi_{g}\left(i-1 / 2, \mu_{n}\right)}{h} \\
\psi_{g}(x, \mu)=\frac{\psi_{g}\left(i+1 / 2, \mu_{n}\right)+\psi_{g}\left(i-1 / 2, \mu_{n}\right)}{2}
\end{gathered}
$$

where $h=x_{i+1 / 2}-x_{i-1 / 2}=\Delta x_{i}$.

Then, including Eqs.3.89, 3.93 and 3.94 for each direction $n, 3.95$ is obtained which is the multi-group steady-state Neutron Transport Equation with $S_{N}$ and FDM discretizations Lewis and Miller Jr [1984]:

$$
\begin{aligned}
& \mu_{n} \frac{\psi_{g}\left(i+1 / 2, \mu_{n}\right)-\psi_{g}\left(i-1 / 2, \mu_{n}\right)}{h}+\Sigma_{t, g}(i) \frac{\psi_{g}\left(i+1 / 2, \mu_{n}\right)+\psi_{g}\left(i-1 / 2, \mu_{n}\right)}{2} \\
- & \frac{1}{4} \sum_{g^{\prime}=1}^{G} \sum_{l=0}^{L}(2 l+1) \Sigma_{s, g^{\prime} \rightarrow g, l}(i) P_{l}\left(\mu_{n}\right) \sum_{m=1}^{N} w_{m}\left[\psi_{g^{\prime}}\left(i+1 / 2, \mu_{m}\right)+\psi_{g^{\prime}}\left(i-1 / 2, \mu_{m}\right)\right] P_{l}\left(\mu_{m}\right) \\
= & \frac{1}{k_{e f f}} \frac{\chi_{g}(i)}{4} \sum_{g^{\prime}=1}^{G} \nu \Sigma_{f, g^{\prime}}(i) \sum_{m=1}^{N} w_{m}\left[\psi_{g^{\prime}}\left(i+1 / 2, \mu_{m}\right)+\psi_{g}\left(i-1 / 2, \mu_{m}\right)\right]
\end{aligned}
$$

Where:

$$
\begin{aligned}
& 1 \leq n \leq N \quad \text { with } N \text { the number of discrete directions } \\
& 1 \leq g \leq G \quad \text { with } G \text { the total number of energy groups }
\end{aligned}
$$

$0 \leq l \leq L \quad$ with $L$ the order of the Legendre polynomial scattering expansion $w_{m}$ are the weighting coefficients of directions $\mu_{m}$ $i$ is the mesh cell number 


\subsection{D steady state Neutron Transport Equation with Anisotropic Scattering}

The two-dimensional steady-state Neutron Transport Equation can be expressed as:

$$
\begin{gathered}
\mu \frac{\partial \psi(x, y, \hat{\Omega}, E)}{\partial x}+\eta \frac{\partial \psi(x, y, \hat{\Omega}, E)}{\partial y}+\Sigma_{t}(x, y, E) \psi(x, y, \hat{\Omega}, E)= \\
\quad \int_{0}^{\infty} \int_{4 \pi} \Sigma_{s}\left(x, y, \hat{\Omega}^{\prime} \rightarrow \hat{\Omega}, E^{\prime} \rightarrow E\right) \psi\left(x, y, \hat{\Omega}^{\prime}, E^{\prime}\right) d \hat{\Omega}^{\prime} d E^{\prime} \\
+\frac{1}{k_{\text {eff }}} \frac{\chi(x, y, E)}{4 \pi} \int_{0}^{\infty} \int_{4 \pi} \nu \Sigma_{f}\left(x, y, E^{\prime}\right) \psi\left(x, y, \hat{\Omega}^{\prime}, E^{\prime}\right) d \hat{\Omega}^{\prime} d E^{\prime}
\end{gathered}
$$

Where

$$
\begin{aligned}
& x, y: \text { Spatial variables } \\
& \theta: \text { Polar angle } \\
& \mu: \cos (\theta) \\
& \eta: \sqrt{\left(1-\mu^{2}\right)} \cos (\varphi) \\
& \xi: \sqrt{1-\mu^{2}-\eta^{2}} \\
& \varphi: \text { Azimuthal angle } \\
& \hat{\Omega}=\mu \vec{i}+\eta \vec{j}+\xi \vec{k}: \text { Energy } \\
& \psi(x, y, \hat{\Omega}, E): \text { Angular neutron flux } \\
& \Sigma_{t}(x, y, E): \text { Macroscopic total cross section } \\
& \Sigma_{s}\left(x, y, \hat{\Omega}^{\prime} \rightarrow E^{\prime} \rightarrow E\right): \text { Macroscopic scattering cross section } \\
& \text { from energy } E^{\prime} \text { to } E \text { and from direction } \hat{\Omega}^{\prime} \text { to } \hat{\Omega} \\
& \chi(x, y, E): \text { Fission spectrum } \\
& \Sigma_{f}\left(x, y, E^{\prime}\right): \text { Macroscopic nu-fission cross section }
\end{aligned}
$$

Similarly to the one-dimensional case, the multi-group equation takes the form: 


$$
\begin{aligned}
\mu \frac{\partial}{\partial x} \int_{g} \psi(x, y, \hat{\Omega}, E) d E+\eta \frac{\partial}{\partial y} \int_{g} \psi(x, y, \hat{\Omega}, E) d E+\int_{g} \Sigma_{t}(x, y, E) \psi(x, y, \hat{\Omega}, E) d E= \\
\quad \sum_{g^{\prime}=1}^{G} \int_{g} \int_{g^{\prime}} \int_{4 \pi} \Sigma_{s}\left(x, y, \hat{\Omega}^{\prime} \rightarrow \hat{\Omega}, E^{\prime} \rightarrow E\right) \psi\left(x, y, \hat{\Omega}^{\prime}, E^{\prime}\right) d \hat{\Omega}^{\prime} d E^{\prime} d E \\
+\frac{1}{k_{e f f}} \int_{g} \frac{\chi(x, y, E)}{4 \pi} \sum_{g^{\prime}=1}^{G} \int_{g^{\prime}} \int_{4 \pi} \nu\left(x, y, E^{\prime}\right) \Sigma_{f}\left(x, y, E^{\prime}\right) \psi\left(x, y, \hat{\Omega}^{\prime}, E^{\prime}\right) d \hat{\Omega}^{\prime} d E^{\prime} d E
\end{aligned}
$$

And introducing the multi-group cross sections and neutron fluxes:

$$
\begin{aligned}
\mu \frac{\partial}{\partial x} \psi_{g}(x, y, \hat{\Omega}) & +\eta \frac{\partial}{\partial y} \psi_{g}(x, y, \hat{\Omega})+\Sigma_{t, g}(x, y) \psi_{g}(x, y, \hat{\Omega})= \\
& \sum_{g^{\prime}=1}^{G} \int_{4 \pi} \Sigma_{s, g^{\prime} \rightarrow g}\left(x, y, \hat{\Omega}^{\prime} \rightarrow \hat{\Omega}\right) \psi_{g^{\prime}}\left(x, y, \hat{\Omega}^{\prime}\right) d \hat{\Omega}^{\prime} \\
& \left.+\frac{1}{k_{e f f}} \frac{\chi_{g}(x, y)}{4 \pi} \sum_{g^{\prime}=1}^{G} \int_{4 \pi} \nu\right) \Sigma_{f, g^{\prime}}(x, y) \psi_{g^{\prime}}\left(x, y, \hat{\Omega}^{\prime}\right) d \hat{\Omega}
\end{aligned}
$$

Similar to one-dimensional case the scalar flux can be approximated by quadrature formula:

$$
\phi(x, y)=\int_{0}^{2 \pi} \int_{-1}^{1} \psi(x, y, \mu, \varphi) d \mu d \varphi=\frac{1}{8} \sum_{n=1}^{N} w_{n} \psi\left(x, y, \mu_{n}, \varphi_{n}\right)=\frac{1}{8} \sum_{n=1}^{N} w_{n} \psi\left(x, y, \mu_{n}, \eta_{n}\right)
$$

From here, the scattering term will be dealt with separately. Eq.3.100 is obtained by applying the discrete ordinates method to Eq.3.98.

$$
\begin{gathered}
\mu_{n} \frac{\partial \psi_{g}\left(x, y, \mu_{n}, \eta_{n}\right)}{\partial x}+\eta_{n} \frac{\partial \psi_{g}\left(x, y, \mu_{n}, \eta_{n}\right)}{\partial y}+\Sigma_{t, g}(x, y, E) \psi_{g}\left(x, y, \mu_{n}, \eta_{n}\right)= \\
q_{s}(x, y)+\frac{1}{k_{e f f}} \frac{\chi_{g}(x, y)}{8} \sum_{g^{\prime}=1}^{G} \nu \Sigma_{f, g^{\prime}}(x, y) \sum_{m=1}^{N} w_{m} \psi_{g^{\prime}}\left(x, y, \mu_{m}, \eta_{m}\right)
\end{gathered}
$$


Where $q_{s}(x, y)$ term can be, as in the one-dimensional case, expanded with Legendre Polinomials, but in this case, it is also necessary to use an Spherical Harmonics Expansion for the angular flux defined in terms of the Associated Legendre Polynomials [Lewis and Miller Jr, 1984, Bell and Glasstone, 1985]. Then, the $q_{s}$ term is expressed as:

$$
\begin{gathered}
q_{s}(x, y)=\frac{1}{8} \sum_{g^{\prime}=1}^{G} \sum_{l=0}^{L}(2 l+1) \Sigma_{s, g^{\prime} \rightarrow g, l}(x, y)\left\{P_{l}\left(\mu_{n}\right) \sum_{m=1}^{N} w_{m} P_{l}\left(\mu_{m}\right) \psi_{g^{\prime}}\left(x, y, \mu_{m} \eta_{m}\right)\right. \\
+2 \sum_{k=1}^{l} \frac{(l-k) !}{(l+k) !} P_{l}^{k}\left(\mu_{n}\right)\left[\sum_{m=1}^{N} w_{m} P_{l}^{k}\left(\mu_{m}\right) \psi_{g^{\prime}}\left(x, y, \mu_{m}, \eta_{m}\right) \cos \left(k \varphi_{m}\right) \cos \left(k \varphi_{n}\right)\right. \\
\left.\left.\quad+\sum_{m=1}^{N} w_{m} P_{l}^{k}\left(\mu_{m}\right) \psi_{g^{\prime}}\left(x, y, \mu_{m}, \eta_{m}\right) \sin \left(k \varphi_{m}\right) \sin \left(k \varphi_{n}\right)\right]\right\}
\end{gathered}
$$

Scattering and fission terms are divided by 8 because the weights corresponding to each octant are normalized to sum 1. Rearranging terms as explained in Bernal García [2018], the scattering term is simplified as:

$$
q_{s}(x, y)=\frac{1}{8} \sum_{g^{\prime}=1}^{G} \sum_{m=1}^{N} w_{m} \psi_{g^{\prime}}\left(x, y, \mu_{m}, \eta_{m}\right) \cdot \Sigma_{s, g^{\prime} \rightarrow g, L, n, m}(x, y)
$$

Where

$$
\begin{aligned}
\Sigma_{s, g^{\prime} \rightarrow g, L, n, m}(x, y) & =\sum_{l=0}^{L}(2 l+1) \Sigma_{s, g^{\prime} \rightarrow g, l}(x, y)\left\{P_{l}\left(\mu_{n}\right) P_{l}\left(\mu_{m}\right)\right. \\
& \left.+2 \sum_{k=1}^{l} \frac{(l-k) !}{(l+k) !} P_{l}^{k}\left(\mu_{n}\right) P_{l}^{k}\left(\mu_{m}\right)\left[\cos \left(k\left(\varphi_{n}-\varphi_{m}\right)\right)\right]\right\}
\end{aligned}
$$

To reduce the number of equations by half without loss of precision, the author used the property of symmetry for the case of 2D plane geometry with respect to the polar angle. This can be demonstrated if one can consider that $\mu_{1}=\mu_{2}$ and $\varphi_{1}=-\varphi_{2}$ due to the z-axis symmetry explained at Fig.3.11

For the previous two directions, one can sum the terms $\Sigma_{s, g^{\prime} \rightarrow g, L, n, m}$ as in Eq.3.104 


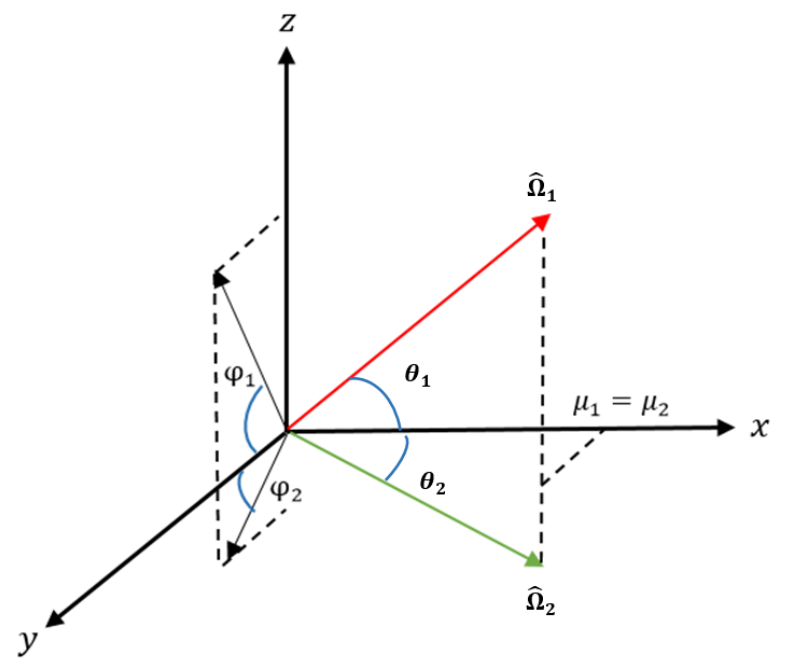

Figure 3.11: Symmetry.

$$
\begin{aligned}
& \Sigma_{s, g^{\prime} \rightarrow g, L, n, 1}(x, y)+\Sigma_{s, g^{\prime} \rightarrow g, L, n, 2}(x, y) \\
& =\sum_{l=0}^{L}(2 l+1) \Sigma_{s, g^{\prime} \rightarrow g, l}(x, y)\left\{P _ { l } ( \mu _ { n } ) \left[P_{l}\left(\mu_{1}\right)+\left[P_{l}\left(\mu_{2}\right)\right]\right.\right. \\
& \left.+2 \sum_{k=1}^{l} \frac{(l-k) !}{(l+k) !} P_{l}^{k}\left(\mu_{n}\right)\left[P_{l}^{k}\left(\mu_{1}\right)\left[\cos \left(k\left(\varphi_{n}-\varphi_{1}\right)\right)\right]+P_{l}^{k}\left(\mu_{2}\right)\left[\cos \left(k\left(\varphi_{n}-\varphi_{2}\right)\right)\right]\right]\right\} \\
& \quad=\sum_{l=0}^{L}(2 l+1) \Sigma_{s, g^{\prime} \rightarrow g, l}(x, y)\left\{P_{l}\left(\mu_{n}\right)\left[2 P_{l}\left(\mu_{1}\right)\right]\right. \\
& \left.+2 \sum_{k=1}^{l} \frac{(l-k) !}{(l+k) !} P_{l}\left(\mu_{n}\right) P_{l}^{k}\left(\mu_{1}\right)\left[\cos \left(k\left(\varphi_{n}-\varphi_{1}\right)\right)\right]+\left[\cos \left(k\left(\varphi_{n}+\varphi_{1}\right)\right)\right]\right\}
\end{aligned}
$$

Using the trigonometry relation $\cos (x \pm y)=\cos (x) \cos (y) \mp \sin (x) \sin (y)$ the Eq.3.104 is simplified as scattering term considering the whole unit sphere (3D). The angular flux of both directions, 1 and 2, will be the same because they are symmetric. Then, the sum of $\Sigma_{s, g^{\prime} \rightarrow g, L, n, 1}(x, y)$ and $\Sigma_{s, g^{\prime} \rightarrow g, L, n, 2}(x, y)$ can be condensed in $\Sigma_{s, g^{\prime} \rightarrow g, L, n, m}(x, y)^{3 D}$. 


$$
\begin{aligned}
\Sigma_{s, g^{\prime} \rightarrow g, L, n, m}(x, y)^{3 D} & =2 \sum_{l=0}^{L}(2 l+1) \Sigma_{s, g^{\prime} \rightarrow g, l}(x, y)\left\{P_{l}\left(\mu_{n}\right)\left[P_{l}\left(\mu_{1}\right)\right]\right. \\
+ & \left.2 \sum_{k=1}^{l} \frac{(l-k) !}{(l+k) !} P_{l}^{k}\left(\mu_{n}\right) P_{l}^{k}\left(\mu_{1}\right)\left[\cos \left(k \varphi_{n}\right) \cos \left(k \varphi_{1}\right)\right]\right\}
\end{aligned}
$$

Therefore, if one wants only to consider a unit semi-sphere (2D):

$$
\begin{aligned}
\Sigma_{s, g^{\prime} \rightarrow g, L, n, m}(x, y)^{2 D} & =\frac{\Sigma_{s, g^{\prime} \rightarrow g, L, n, m}(x, y)^{3 D}}{2}= \\
& \sum_{l=0}^{L}(2 l+1) \Sigma_{s, g^{\prime} \rightarrow g, l}(x, y)\left\{P_{l}\left(\mu_{n}\right)\left[P_{l}\left(\mu_{m}\right)\right]\right. \\
& \left.+2 \sum_{k=1}^{l} \frac{(l-k) !}{(l+k) !} P_{l}^{k}\left(\mu_{n}\right) P_{l}^{k}\left(\mu_{m}\right)\left[\cos \left(k \varphi_{n}\right) \cos \left(k \varphi_{m}\right)\right]\right\}
\end{aligned}
$$

Note that for the new formulation the consideration of 8 octants changed to 4, since only the positive polar angles are considered. Then, the sum of weighting coefficients is 4 and the $q_{s}$ term and fission term will be divided now by 4 .

$$
q_{s}^{2 D}(x, y)=\frac{1}{4} \sum_{g^{\prime}=1}^{G} \sum_{m=1}^{N^{\prime}} w_{m} \psi_{g^{\prime}}\left(x, y, \mu_{m}, \eta_{m}\right) \cdot \Sigma_{s, g^{\prime} \rightarrow g, L, n, m}(x, y)^{2 D}
$$

\subsubsection{Finite Difference Method for 2D}

Therefore, Eq.3.108 is the 2D version of the Eq.3.100.

$$
\begin{gathered}
\mu_{n} \frac{\partial \psi_{g}\left(x, y, \mu_{n}, \eta_{n}\right)}{\partial x}+\eta_{n} \frac{\partial \psi_{g}\left(x, y, \mu_{n}, \eta_{n}\right)}{\partial y}+\Sigma_{t, g}(x, y, E) \psi_{g}\left(x, y, \mu_{n}, \eta_{n}\right)= \\
q_{s}^{2 D}(x, y)+\frac{1}{k_{e f f}} \frac{\chi_{g}(x, y)}{4} \sum_{g^{\prime}=1}^{G} \nu \Sigma_{f, g^{\prime}}(x, y) \sum_{m=1}^{N^{\prime}} w_{m} \psi_{g^{\prime}}\left(x, y, \mu_{m}, \eta_{m}\right)
\end{gathered}
$$


With this formulation, the number of considered directions is the half of directions than considering the whole unit sphere. Consequently, the number of equations is reduced by half, $N^{\prime}=N / 2$.

The Finite Difference Method is used for calculating the spatial derivatives and the cell average value of the angular neutron flux, as shown in Eqs.3.109-3.111. In these equations, $\psi_{i, j}^{n}$ is the angular neutron flux for the direction $n$ and the node $i, j$. Cell centered points are defined by $\left[x_{i}, y_{j}\right]=\left[1 / 2\left(x_{i-1 / 2}+x_{i+1 / 2}\right), 1 / 2\left(y_{j-1 / 2}+\right.\right.$ $\left.\left.y_{j+1 / 2}\right)\right]$. Fig.3.12 shows an example of the numbering of the nodes for each cell where $h$ and $k$ are the half cell distance in axis $x$ and $y$, respectively. An example of the angular approach for $S 2$ is shown in fig.3.13.

$$
\begin{aligned}
& \frac{\partial \psi_{g}\left(x, y, \mu_{n}, \eta_{n}\right)}{\partial x}=\frac{\psi_{i+\frac{1}{2}, j+\frac{1}{2}}^{n}+\psi_{i+\frac{1}{2}, j-\frac{1}{2}}^{n}-\psi_{i-\frac{1}{2}, j+\frac{1}{2}}^{n}-\psi_{i-\frac{1}{2}, j-\frac{1}{2}}^{n}}{2 h} \\
& \frac{\partial \psi_{g}\left(x, y, \mu_{n}, \eta_{n}\right)}{\partial y}=\frac{\psi_{i+\frac{1}{2}, j+\frac{1}{2}}^{n}+\psi_{i-\frac{1}{2}, j+\frac{1}{2}}^{n}-\psi_{i+\frac{1}{2}, j-\frac{1}{2}}^{n}-\psi_{i-\frac{1}{2}, j-\frac{1}{2}}^{n}}{2 k} \\
& \psi_{g}\left(x, y, \mu_{n}, \eta_{n}\right)=\frac{\psi_{i+\frac{1}{2}, j+\frac{1}{2}}^{n}+\psi_{i+\frac{1}{2}, j-\frac{1}{2}}^{n}+\psi_{i-\frac{1}{2}, j+\frac{1}{2}}^{n}+\psi_{i-\frac{1}{2}, j-\frac{1}{2}}^{n}}{4}
\end{aligned}
$$

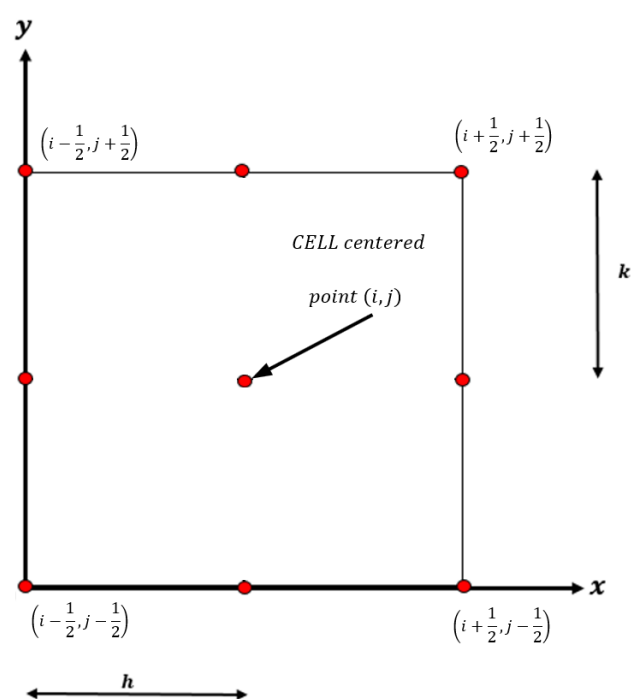

Figure 3.12: Spatial discretization.

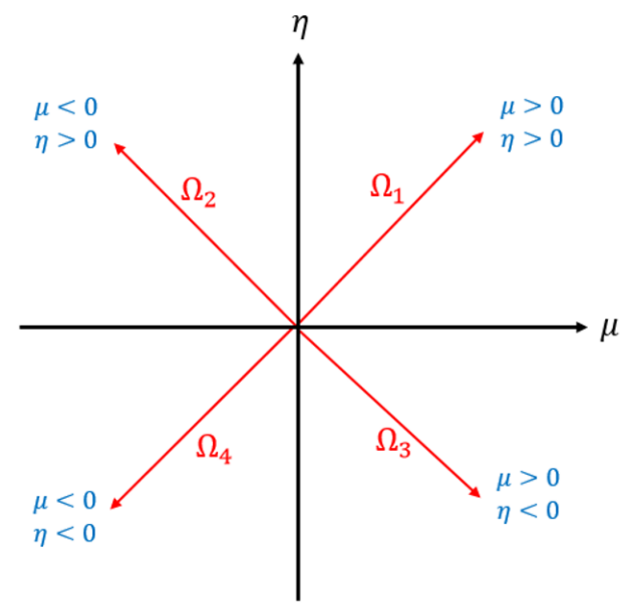

Figure 3.13: 2D angular directions for $S_{2}$. 


\subsubsection{Boundary Conditions for the 2D Discrete Ordinates Method}

Vacuum, reflective and albedo conditions can be expressed similarly to the onedimensional case using eq.3.112 for two-dimensional cases, in which the only difference is the $\beta$ value. It is easy to appreciate that each outcoming flux direction $n$ is function of a complementary direction exiting of our system. Figs.3.14-3.16 show graphically the three expressions presented in eq.3.112.

$$
\begin{aligned}
& \psi_{i, j}^{n}=\beta \cdot \psi_{0, j}^{\frac{N}{2}+1-n} ; 0 \leq j \leq J \text { and }(i=0 \text { or } i=I) \text { for } 1 \leq n \leq \frac{N}{2} \\
& \psi_{i, j}^{n}=\beta \cdot \psi_{0, j}^{\frac{3 N}{2}+1-n} ; 0 \leq j \leq J \text { and }(i=0 \text { or } i=I) \text { for } \frac{N}{2}+1 \leq n \leq N \\
& \psi_{i, j}^{n}=\beta \cdot \psi_{0, j}^{N+1-n} ; 0 \leq i \leq I \text { and }(j=0 \text { or } j=J) \text { for } 1 \leq n \leq N
\end{aligned}
$$

such that $\Omega_{n} \cdot \vec{n}<0$

$$
\beta=0 \text { (vacuum condition) ; } \beta=1 \text { (reflective condition) ; }
$$

$$
\beta \in(0,1) \text { (albedo condition) }
$$

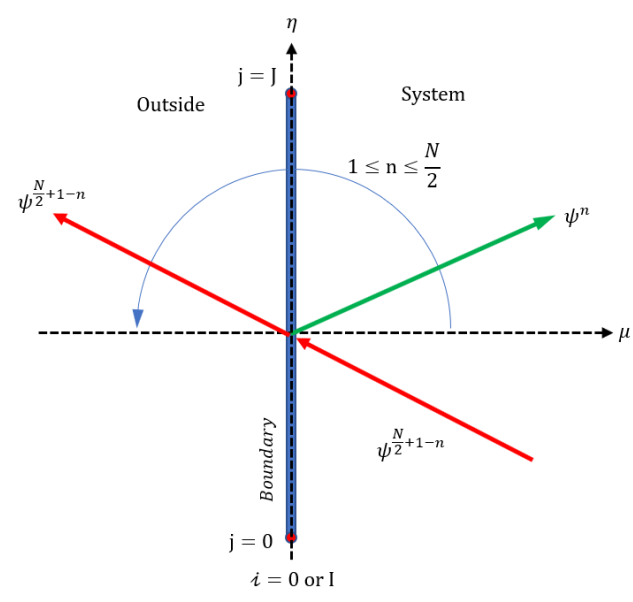

Figure 3.14: Vertical boundary condition with Figure 3.15: Vertical boundary condition with $1 \leq n \leq \frac{N}{2}$.

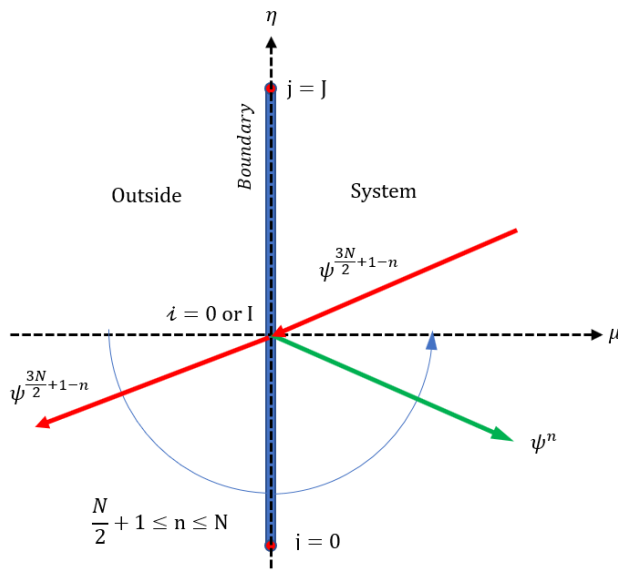

$\frac{N}{2}+1 \leq n \leq N$. 


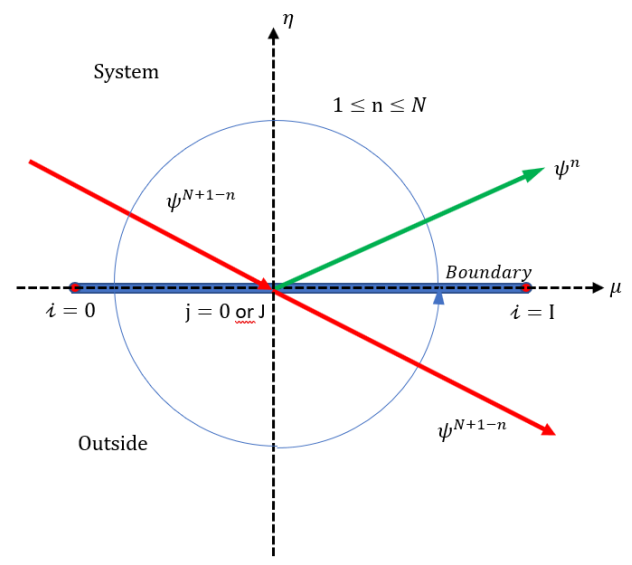

Figure 3.16: Horizontal boundary condition.

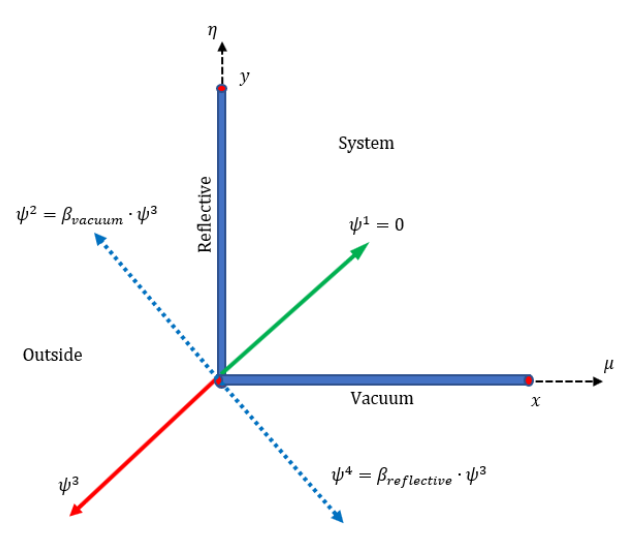

Figure 3.17: Corner boundary condition.

Due to the formulation developed, although the equations are made for each cell, the fluxes appearing inside the equations are considered in nodes or vertex. So, the author recommend some important details to introduce the boundary conditions in a corner where two different boundary conditions, vacuum and reflective converge at this point. Next consideration could not be found in literature by the author for finite difference methods and it was developed by testing the code and thinking in geometry assumptions. Fig.3.17 shows how the boundary conditions in a corner have been considered for a simple $S_{2}$ case.

The Periodic boundary condition can be defined by Eq.3.113.

$$
\begin{array}{lll}
\psi_{0, j}^{n}=\psi_{I, j}^{n} & \text { with } 0 \leq j \leq J & 1 \leq n \leq N \\
\psi_{i, 0}^{n}=\psi_{i, J}^{n} & \text { with } 0 \leq i \leq I & 1 \leq n \leq N
\end{array}
$$

\subsection{Angular Quadratures}

The discrete ordinates method $S_{n}$ is commonly used in nuclear engineering to calculate a numerical solution of the integro-differential form of the Boltzmann transport equation. Basically, the method consist in a numerical integration based 
on collocation points, which are the discrete directions and their weights. The combination of discrete angular directions and weights is called quadrature set.

Carlson and Lathrop proposed to use specialized quadrature sets [Lathrop and Carlson, 1964, Carlson, 1971], that satisfy higher order moments of the direction cosines. For one dimensional cases, the scattering term only depends on one angular variable (polar angle or its direction cosine $\mu$ ). This work analyzes the following Quadrature Sets for one-dimensional cases: Gauss-Legendre, Chebyshev type 1, and Chebyshev type 2. For two dimensional cases the following Quadrature Sets are studied: $L Q_{N}$ (Level-Symmetric), $P_{n}-E W$ (Gauss-Legendre Equal Weight), $P_{n}-T_{n}$ (Gauss-Legendre Chebyshev) [Carlson, 1970, Wai and Gottlieb, 1993, Walters, 1987] and a new developed $P Q$ Product Quadrature [Bernal et al.].

All the quadratures mentioned were implemented in the code with the capability of using any order number, except in the case of the Level- Symmetric quadrature which only can be developed up to $S_{20}$ due to its limitation.

It is important to highlight that the quadrature sets can have a great influence on the accuracy of the method and it is important the understanding of the quadratures theory to be able to implement these quadratures in the Discrete Ordinates method. Node and weights computed were found to be highly accurate, allowing one to perform quadratures without using standard tables of nodes and weights, saving time and avoiding the risk of error in entering the nodes and weights from tables.

\subsubsection{Gauss-Legendre}

There are several quadrature rules for numerical integration methods but one powerful tool for approximating integrals is Gaussian Quadrature. In 1D slab, the transport equation is function of only one direction cosine. So one can integrate a function $f(x)$ by means of the following equation:

$$
\int_{-1}^{1} f(x) d x \approx \sum_{i=1}^{N} w_{i} \cdot f(x i)
$$

The collocation points $x_{i}$ are the roots of the Legendre polynomials and $w_{i}$ the weights which can be calculated by: 


$$
w_{i}=\frac{2}{\left(1-x_{i}^{2}\right)\left[P_{N}^{\prime}\left(x_{i}\right)\right]^{2}}=\frac{2\left(1-x_{i}^{2}\right)}{(N+1)^{2}\left[P_{N+1}\left(x_{i}\right)\right]^{2}}
$$

Gaussian integration is based on the use of polynomials to approximate the integrand $f(x)$ over the interval $[-1,1]$. The coefficients of these polynomials are unknown variables which can be determined by using suitable methods. A uniform weighting over the interval is achieved by the Gaussian quadrature.

Although the method was developed for the interval $[-1,1]$ the method can be extended to [a,b]. To do this, a simple way is linearly map this interval for $x_{i}$ onto the $[-1,1]$ interval for $f(x)$. So, the new collocation points $x_{i}^{\prime}$ are calculated as:

$$
x_{i}^{\prime}=\frac{1}{2}(a+b)+\frac{1}{2}(b-a) \cdot x_{i}
$$

The hight accuracy of Gaussian quadrature is due to the fact that it integrates very-high-degree polynomials exactly. Other methods like trapezoidal method and Simpson's rule use a predetermined fixed grid points. In the Gaussian quadrature, both grid points and the weights are chosen to maximize the degree of accuracy.

\subsubsection{Chebyshev type 1 and type 2}

The Chebyshev polynomials of the first type $T_{n}(x)$ are a set of othogonal polynomials defined as the solution of the Chebyshev differential equation.

The points and their corresponding weights for the Chebyshev type 1 can be calculated by:

$$
x_{i}=\cos \left(\frac{2 i-1}{2 N} \pi\right) \quad w_{i}=\frac{\pi}{N}
$$

For the Chebyshev type 2 the formulation is:

$$
x_{i}=\cos \left(\frac{i}{N+1} \pi\right) \quad w_{i}=\frac{\pi}{N+1} \sin ^{2}\left(\frac{i}{N+1} \pi\right)
$$




\subsubsection{Level Symmetric, $L Q_{N}$}

One of the strengths of the Level-Symmetric quadrature sets is that it conserves the symmetry of the eight octants with respect to $\pi / 2$ rotations selecting the corresponding points on the unit sphere [Hébert, 2009].

So, in this technique, the directions are ordered on each octant of the unit sphere along z-axis $(\xi)$ on $N / 2$ distinct level, being the number of directions per level equal to:

$$
\frac{N}{2}-i+1 \quad \text { for } \quad i=1, N / 2 \text {. }
$$

where $N$ is the order of the $S_{N}$ method and the total number of directions for three-dimensional geometries is:

$$
N \cdot(N+2)
$$

Taking into account $|\hat{\Omega}|=1$, that it says $\mu_{i}^{2}+\eta_{j}^{2}+\xi_{k}^{2}=1$ and with $i+j+k=\frac{N}{2}+2$, one can determine the index $i, j, k$ of the direction cosines by the formulation:

$$
\mu_{i}^{2}=\mu_{1}^{2}+(i-1) \frac{2\left(1-3 \mu_{1}^{2}\right)}{(N-2)} \quad \text { for } 2 \leq i \leq N / 2 \text { and } 0<\mu_{1}^{2} \leq 1 / 3
$$

The choice of $\mu_{1}$ determines the distribution of directions on the octant. So, if the value $\mu_{1}$ is small, the directions will be clustered close the poles of the sphere. On the contrary, if the value is large, the points will be located far from the poles. To calculate the weights associated to each point, it is necessary to consider another set of equations, such us those showed in eq.3.122 for the $S_{8}$ levelsymmetric quadrature set: 


$$
\begin{aligned}
2_{p} 1+2 p_{2} & =w_{1}^{\prime} \\
2 p_{2}+p_{3} & =w_{2}^{\prime} \\
2 p_{2} & =w_{3}^{\prime} \\
1 p_{1} & =w_{4}^{\prime}
\end{aligned}
$$

where $p_{i}$ are the point weights and $w_{i}^{\prime}$ the weight associated with each level. An explicative scheme is shown in Fig.3.18. Moreover, some angular sets for different orders using the Level-Symmetric method can be seen in Fig.3.19.

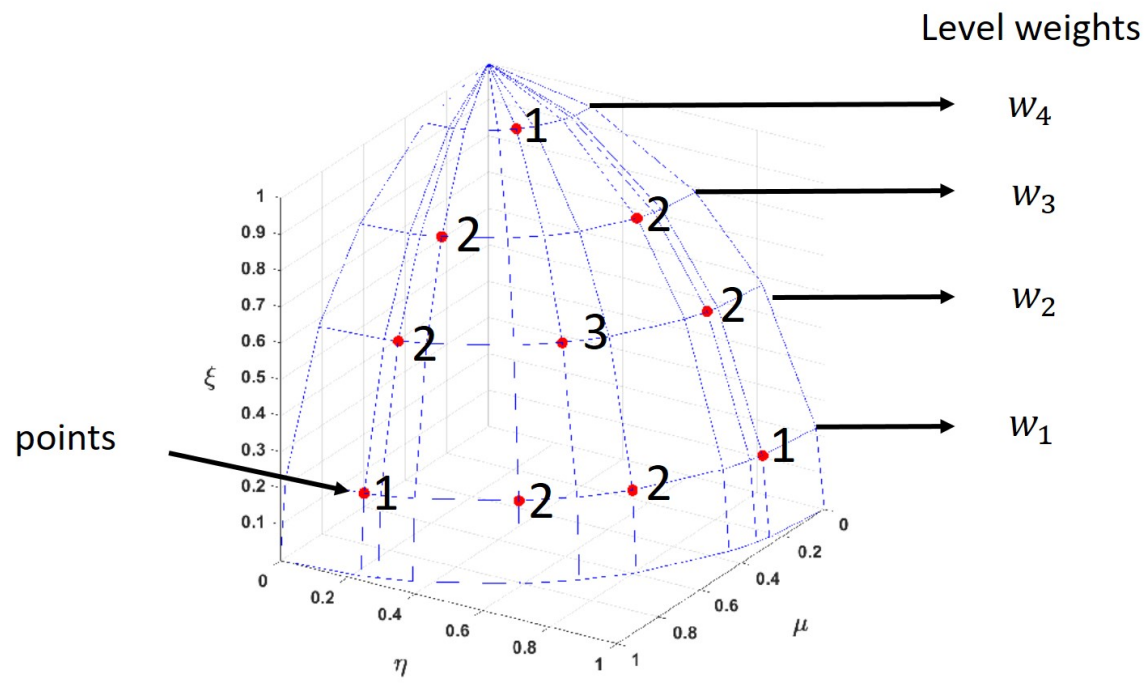

Figure 3.18: Level Symmetric $S_{8}$ example.

Level Symmetric method $L Q_{N}$ is one of the most commonly used techniques for generating quadrature sets, however for order beyond $S_{20}$ the weights are negative which do not make sense. Some problems require higher orders to minimize the ray-effect problems. 


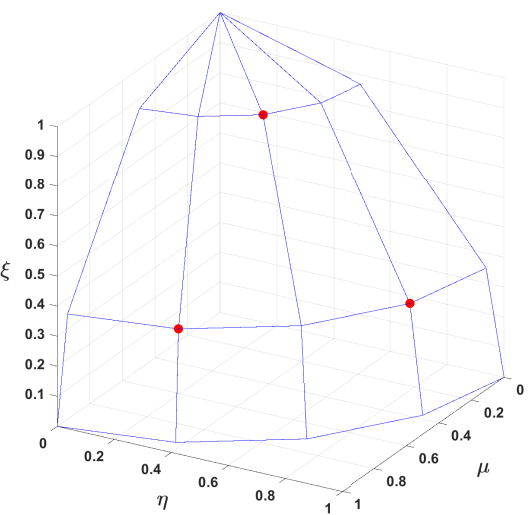

(a) $S_{4}$.

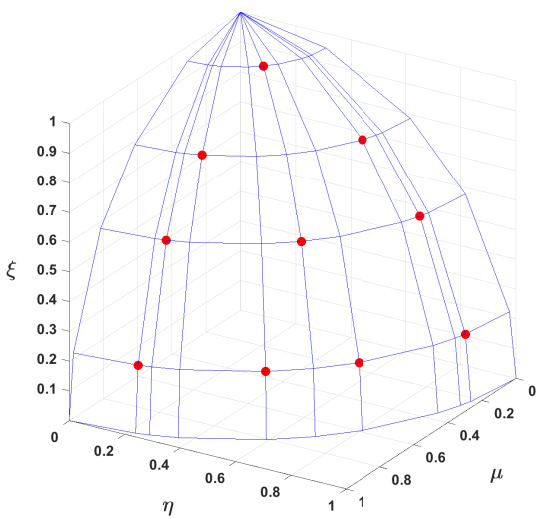

(c) $S_{8}$.

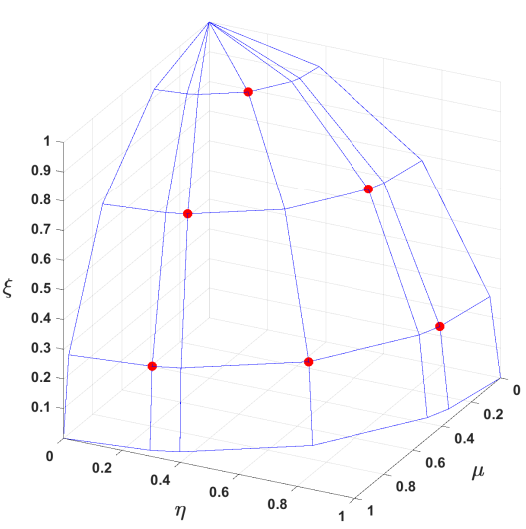

(b) $S_{6}$.

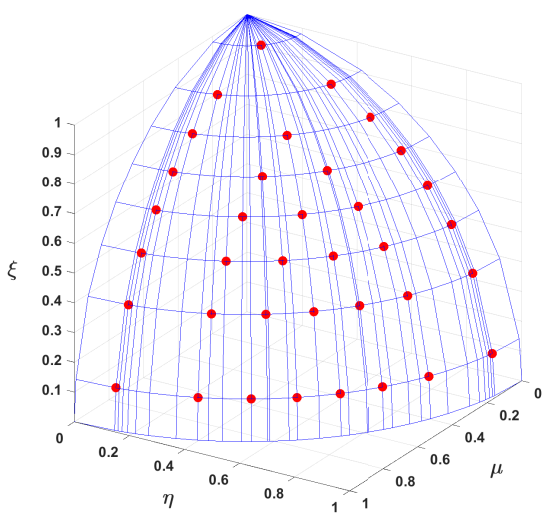

(d) $S_{16}$.

Figure 3.19: Level-Symmetric quadratures for $S_{4}, S_{6}, S_{8}$ and $S_{16}$.

\subsubsection{Gauss-Legendre Equal Weight, $P_{n}-E W$}

The Equal Weight (EW) quadrature set was proposed to avoid obtaining negative weights, which is the fundamental problem of the Level-Symmetric quadrature. The main feature of the equal weight quadratures is to preselect identical weight factors $w_{i}$ for all discrete ordinates to improve the rotational invariance of the quadrature. This quadrature is derived by partitioning the unit sphere into $M$ 
directions, where $M=N(N+2)$ and each direction presents equal weight $w_{i}=$ $1 / M$. The Legendre-Equal Weight $\left(P_{n}-E W\right)$ quadrature uses the Gauss-Legendre quadrature to define the polar angle $\theta$ or $\xi$-levels along the z-axis, while the azimuthal angle is evaluated on each level by dividing the $p i / 2$ angle into $N / 2-$ $i+2$ angular intervals, being $i=1, \ldots, N / 2$.

So, for constructing $N=8$, the number of $\xi$-levels would be 4 and the weights for these levels would be the corresponding Gauss-Legendre quadrature weights $w_{i}$. Hence, the number of direction for each level are $j(i=1, \ldots, 4)=4,3,2,1$ and the weight associated for each level is given by:

$$
w_{i, j}=\frac{w_{i}}{j} \quad \text { for } \quad i=1 \ldots N / 2 \text { and } j(i)=\frac{N}{2}-i+1
$$

An scheme of this example is shown in Fig.3.20. Moreover, some angular sets for different order using $P_{n}$-EW method can be seen in Fig.3.19. Although this technique can be used for any order, the distribution of the points in the azimuthal angle $(\varphi)$ is based on geometric considerations rather than on integration criteria.

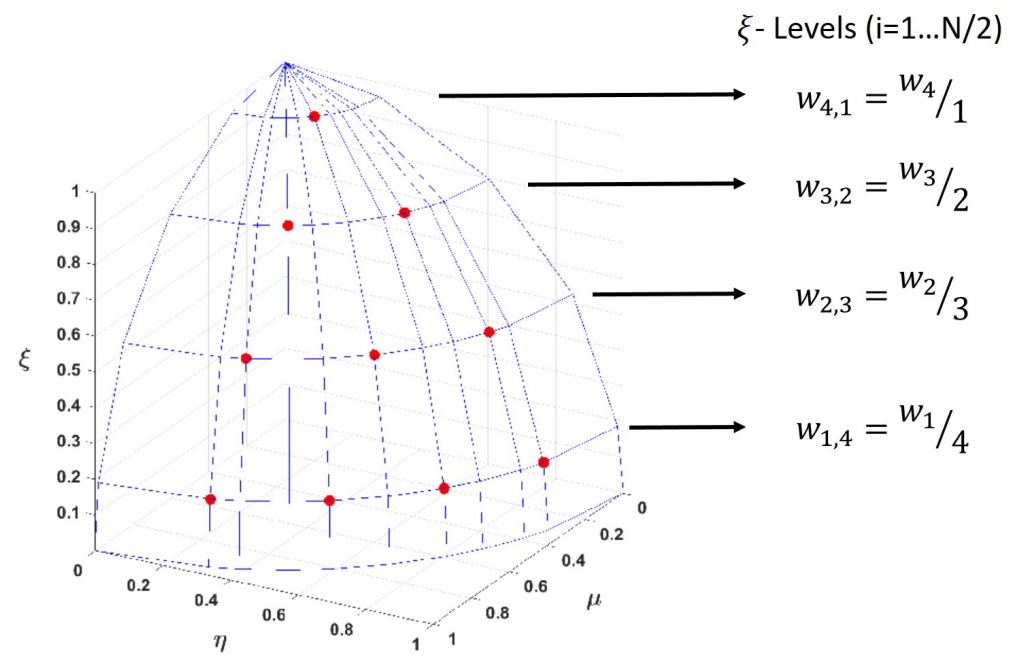

Figure 3.20: $P_{n}-E W$ quadratures for $S_{8}$. 


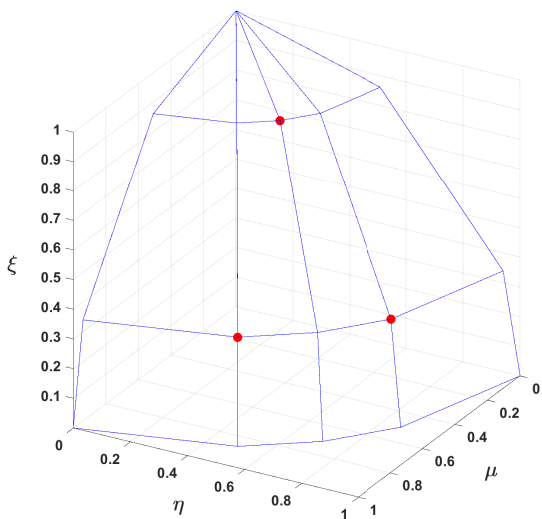

(a) $N=4$.

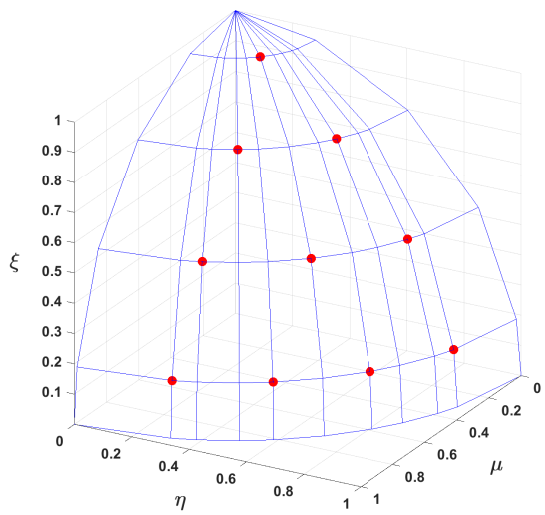

(c) $N=8$.

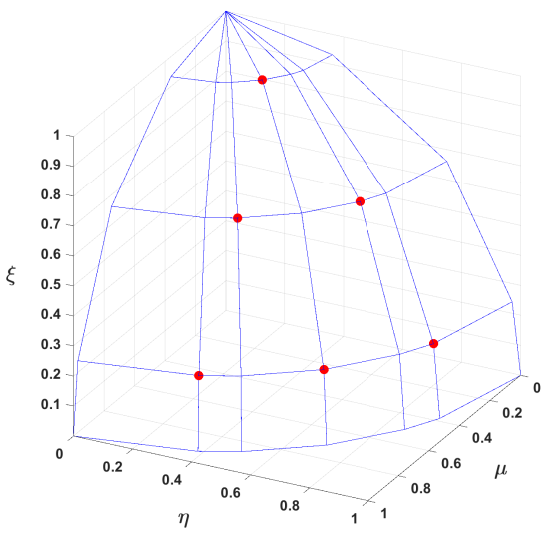

(b) $N=6$.

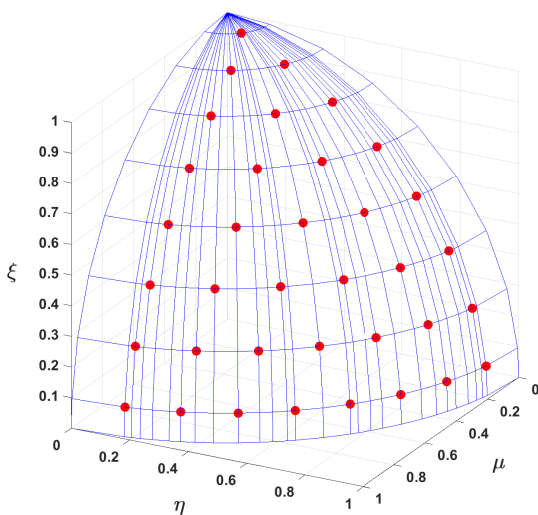

(d) $N=16$.

Figure 3.21: $P_{n}-E W$ quadratures for $S_{4}, S_{6}, S_{8}$ and $S_{16}$.

\subsubsection{Gauss-Legendre Chebyshev, $P_{n}-T_{n}$}

Likewise to the formulation of the $P_{n}-E W$ quadrature, the Gauss-Legendre Chebyshev quadrature chooses the $\xi$-levels along the z-axis by means of the GaussLegendre quadrature formula. However, the azimuthal angle $(\varphi)$ is not equally spaced. The azimuthal angles for each level are defined by the roots of the Chebyshev polynomials of the first type showed in eq.3.117. 
The reason of the good accuracy of this method can be explained considering the integral operator for integration over all solid angle:

$$
\int_{0}^{\pi} \sin \theta d \theta \int_{0}^{2 \pi} d \varphi
$$

If $\cos \theta=\xi$ and $\cos \varphi=y$, then the integral can be expressed as:

$$
2 \int_{-1}^{1} d \xi \int_{-1}^{1} \frac{1}{\sqrt{1-y^{2}}} d y
$$

The integration in the azimuthal angle can be calculated accurately by using the Chebyshev quadrature set. Similarly, the integral in the polar angle can be calculated accurately by the Gauss-Legendre quadrature set.

So, the $P_{n}-T_{n}$ quadrature set defines the azimuthal angles on each level by using the Chebyshev formulation:

$$
y_{i, j}=\cos \varphi_{i, j}=\frac{(2 j-1) \pi}{2 n_{c_{i}}}
$$

where $i$ are the levels of polar angle, $j$ are the subindex for the azimuthal angles and $n_{c_{i}}$ are the total number of angles for each level or the Chebyshev order.

The point weights are calculated in the same way as in the Gauss-Legendre Equal Weight quadrature. 


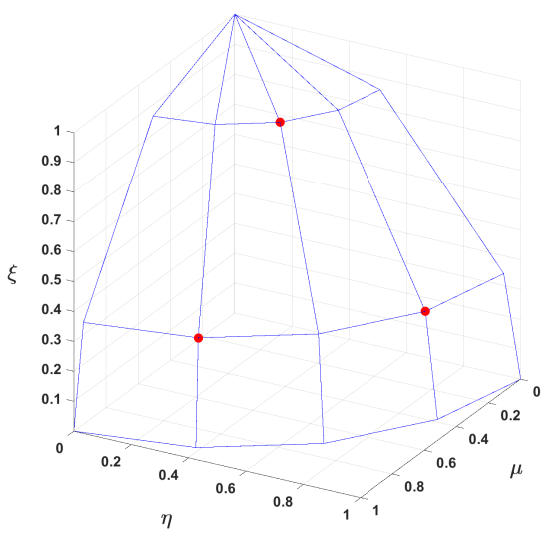

(a) $N=4$.

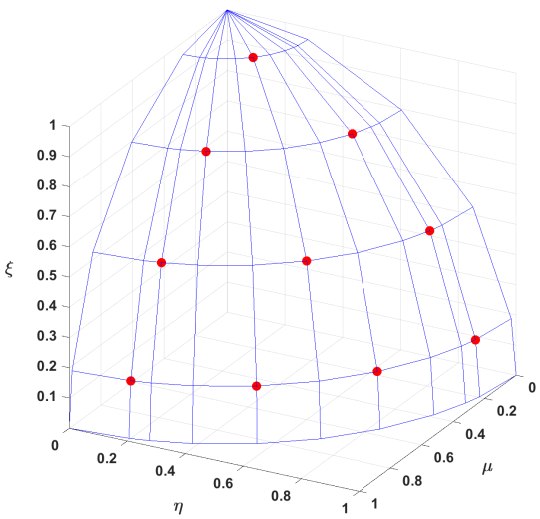

(c) $N=8$.

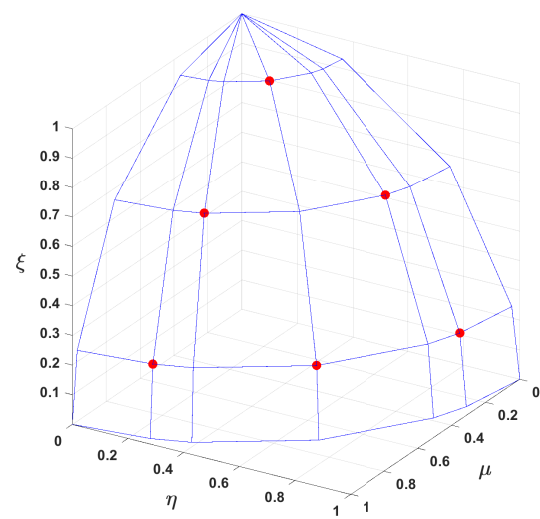

(b) $N=6$.

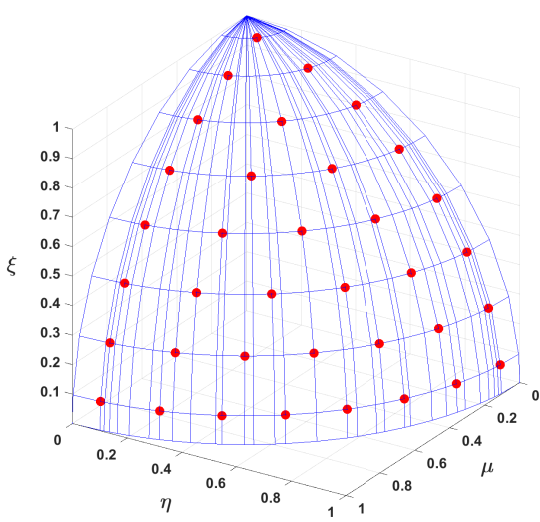

(d) $N=16$.

Figure 3.22: $P_{n}-T_{n}$ quadratures for $S_{4}, S_{6}, S_{8}$ and $S_{16}$.

\subsubsection{Product Quadrature, $P Q$}

The previous quadrature sets use a triangular distribution over the octant. The Product Quadrature $P Q$ was developed by using the Gauss-Legendre quadrature for both angles, polar and azimuthal, and the point weights were calculated as the product of the weights [Bernal et al.]. 




(a) $\left(n^{\circ}\right.$ polar , $n^{\circ}$ azim $)=(2,1)$.

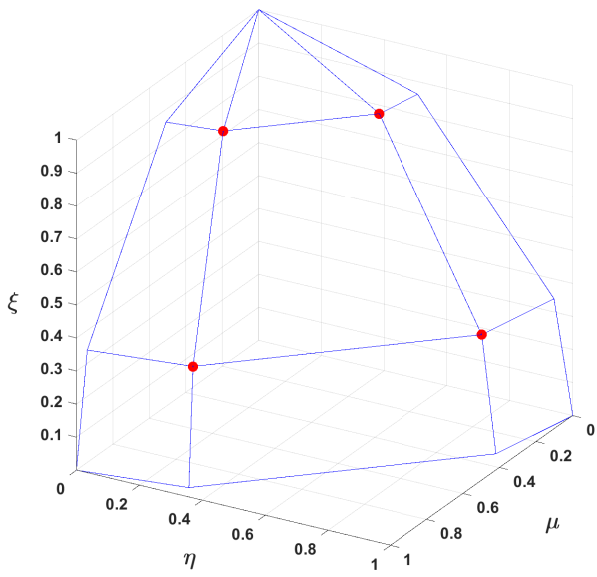

(c) $\left(n^{\circ}\right.$ polar, $n^{\circ}$ azim $)=(2,2)$.

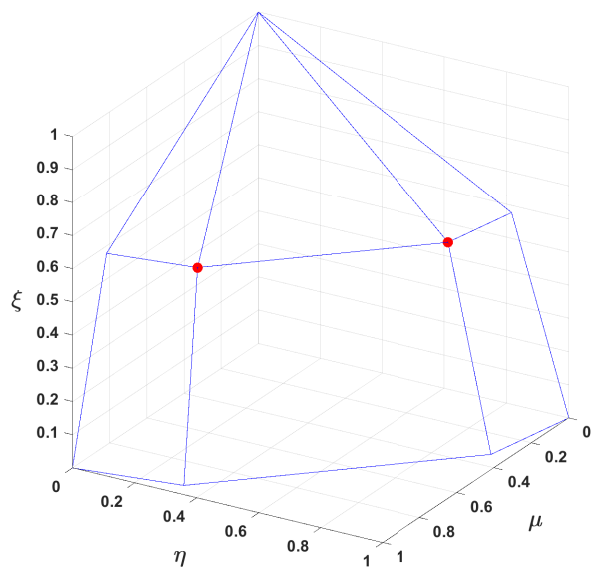

(b) $\left(\mathrm{n}^{\circ}\right.$ polar , $\mathrm{n}^{\circ}$ azim $)=(1,2)$.

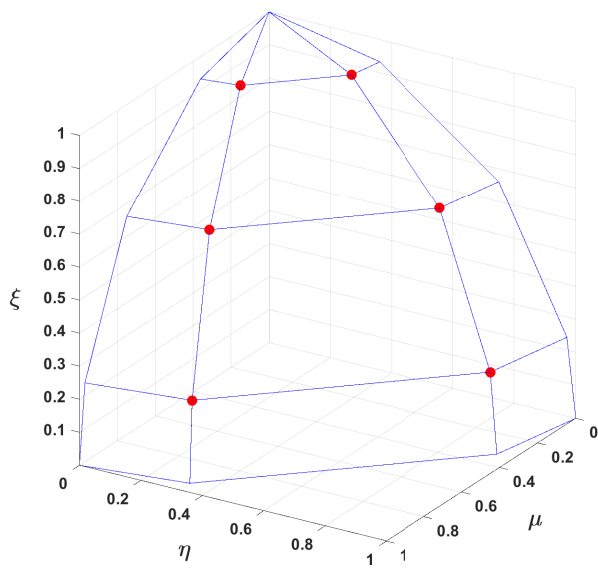

(d) $\left(n^{\circ}\right.$ polar, $n^{\circ}$ azim $)=(3,2)$.

Figure 3.23: $P Q$ quadratures for $\left(\mathrm{n}^{\circ}\right.$ polar, $\mathrm{n}^{\mathrm{o}}$ azim $)=(2,1),(1,2),(2,2),(3,2)$. 


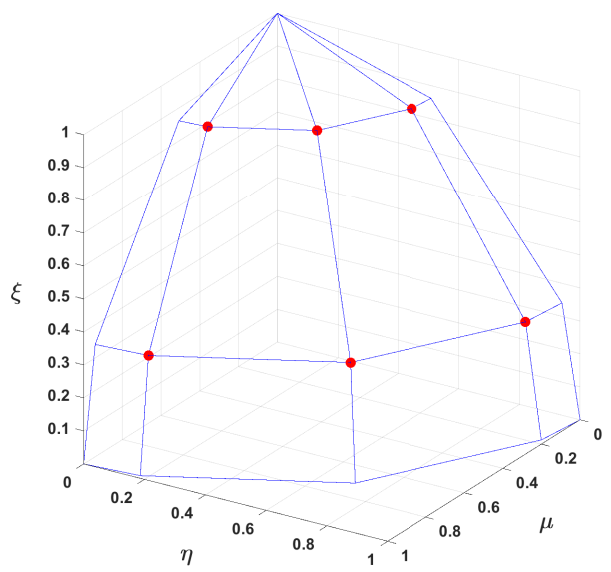

(a) $\left(n^{\circ}\right.$ polar , $n^{\circ}$ azim $)=(2,3)$.

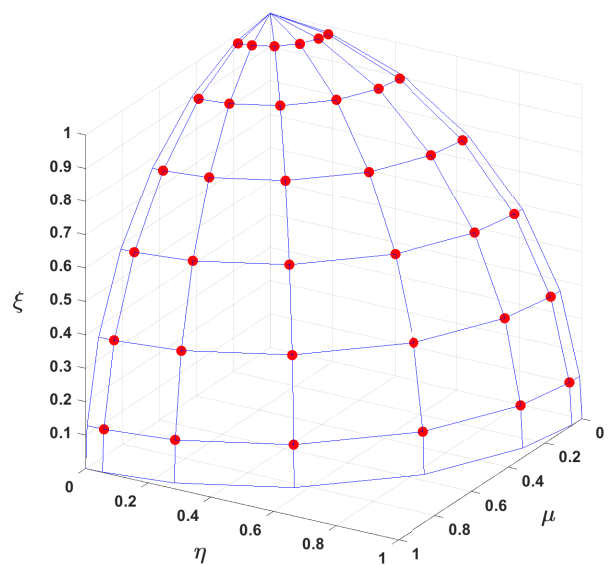

(c) $\left(\mathrm{n}^{\circ}\right.$ polar , $\mathrm{n}^{\mathrm{o}}$ azim $)=(6,6)$.

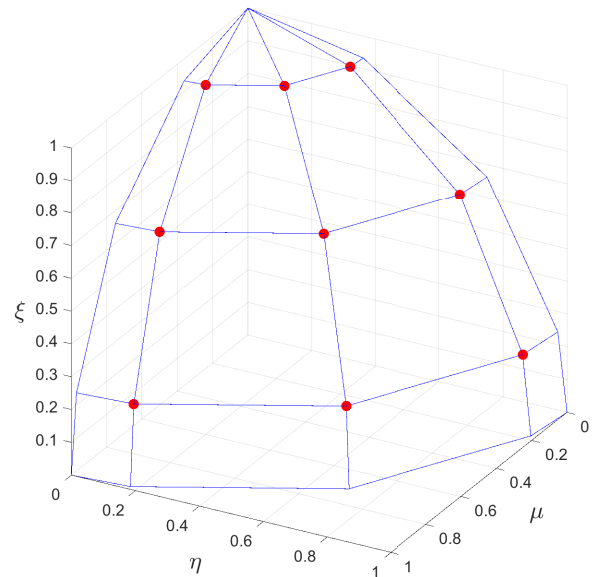

(b) $\left(\mathrm{n}^{\mathrm{o}}\right.$ polar, $\mathrm{n}^{\mathrm{o}}$ azim $)=(3,3)$.

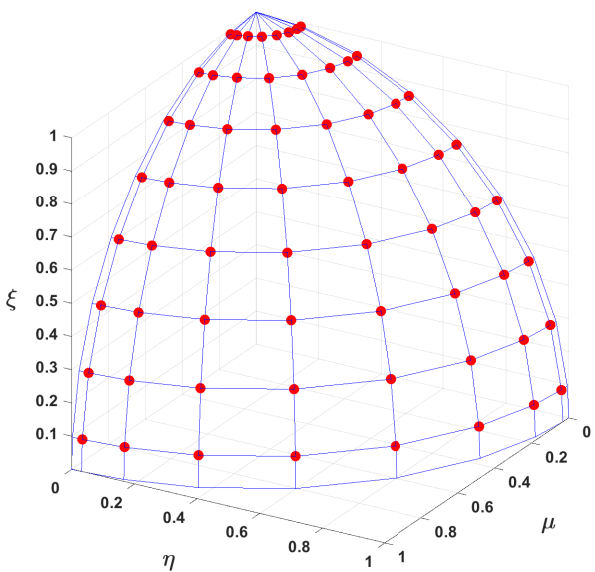

(d) $\left(n^{\circ}\right.$ polar, $n^{\circ}$ azim $)=(8,8)$.

Figure 3.24: $P Q$ quadratures for $\left(\mathrm{n}^{\circ}\right.$ polar, $\mathrm{n}^{\mathrm{o}}$ azim $)=(2,3),(3,3),(6,6),(8,8)$. 


\subsection{Eigenvalue problem}

The eigenvalue problem arising from the neutron transport equation or its different approximations is commonly solved by the Power Iteration method. However, in the nuclear field, the dominance ratio which determine the convergence of the method normally is close to 1 . It is well known that the Power iteration method converges slowly in this case.

This is the reason why Krylov methods can be useful. They permit to solve the eigenvalue problem faster than the Power iteration method when the solution of the problem uses a high dominance ratio. A review of this can be found in Vidal-Ferràndiz et al. [2018a]. In addition, Krylov methods allow the calculation of several eigenvalues ( $k_{e f f}$ and the subcritical eigenvalues). In particular, the Krylov-Schur method is becoming more commonly used in several works related to the nuclear field Bernal et al. [2017]. This research work solves the eigenvalue problem by using the Krylov-Schur algorithm implemented in the SLEPc library. This library called Scalable Libreary for Eigenvalue Problem Computations (SLEPc) is a software library for the solution of large, sparse eigenproblems on parallel computers Hernandez et al. [2005]. SLEPc is the state of the art for calculating eigenproblems of large and sparse matrices like those obtained with the method of this thesis. In addition, SLEPc uses PETSc Balay et al. [2019] (Portable, Extensible Toolkit for Scientific Computation) to extend it with all the necessary functionalities for the solution of eigenvalue problems; which includes matrix operations and the solution of linear systems. However the matrices have ill conditioning in this case and it was necessary to implement a direct method using library MUMPS Gueye et al. [2011] and a complete LU factorization.

The following figures provide examples of the type of matrices obtained by the methodology explained in this chapter. The ordering of equations within the matrix $A$ was:

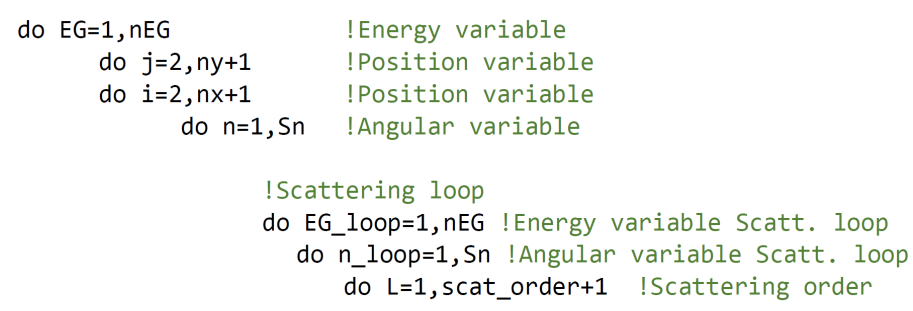

Figure 3.25: Two dimensional ordering scheme of the matrix $A$. 


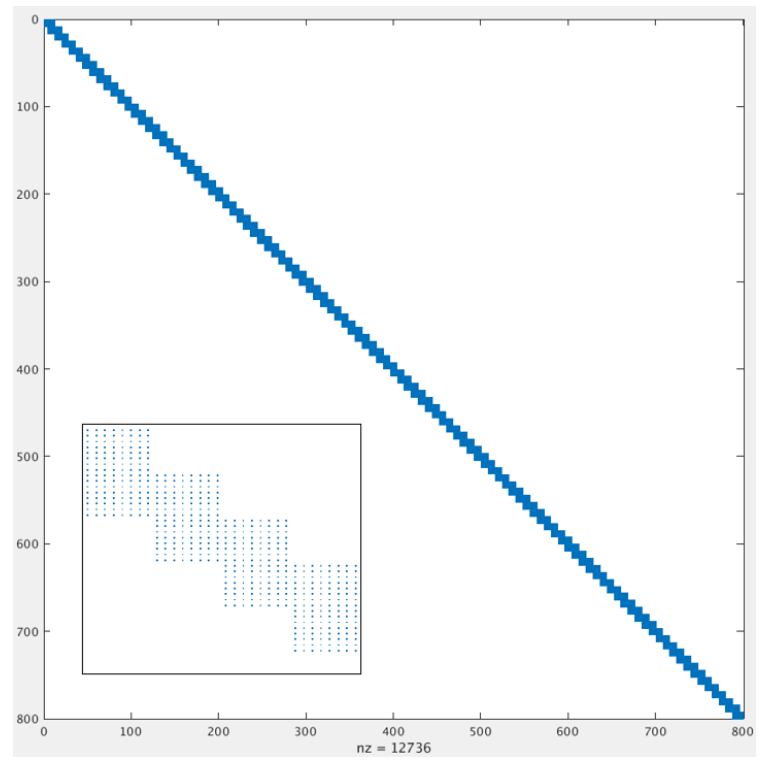

Figure 3.26: Matrix $A, 1 \mathrm{D}$ (one dimension), $1 \mathrm{EG}$ (one energy group), $S_{8}$ (discrete ordinates order 8), 100 cells (discretization).

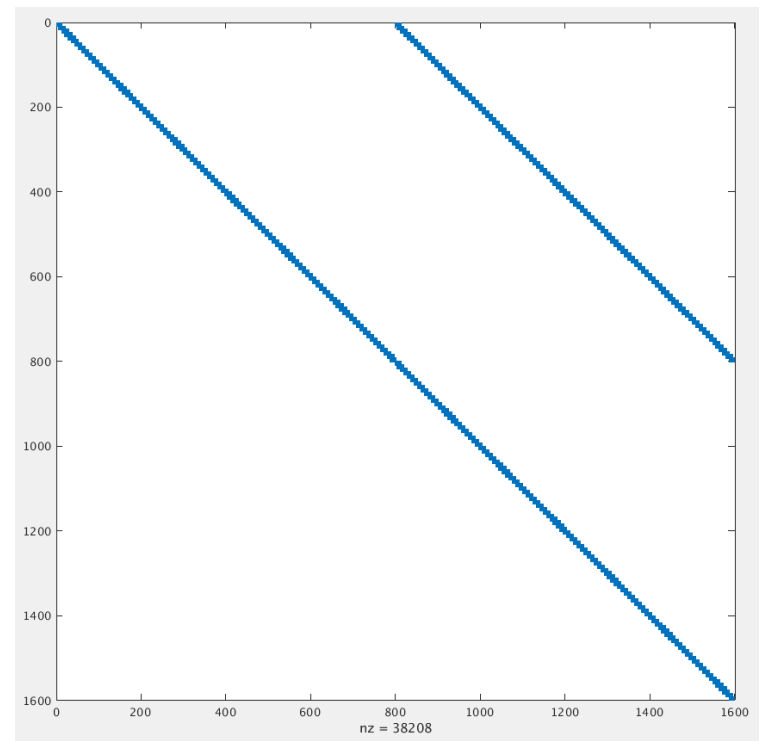

Figure 3.27: Matrix $A, 1 \mathrm{D}$ (one dimension), $2 \mathrm{EG}$ (two energy groups), $S_{8}$ (discrete ordinates order 8), 100 cells (discretization) and no upscattering(the upscattering is not considered). 


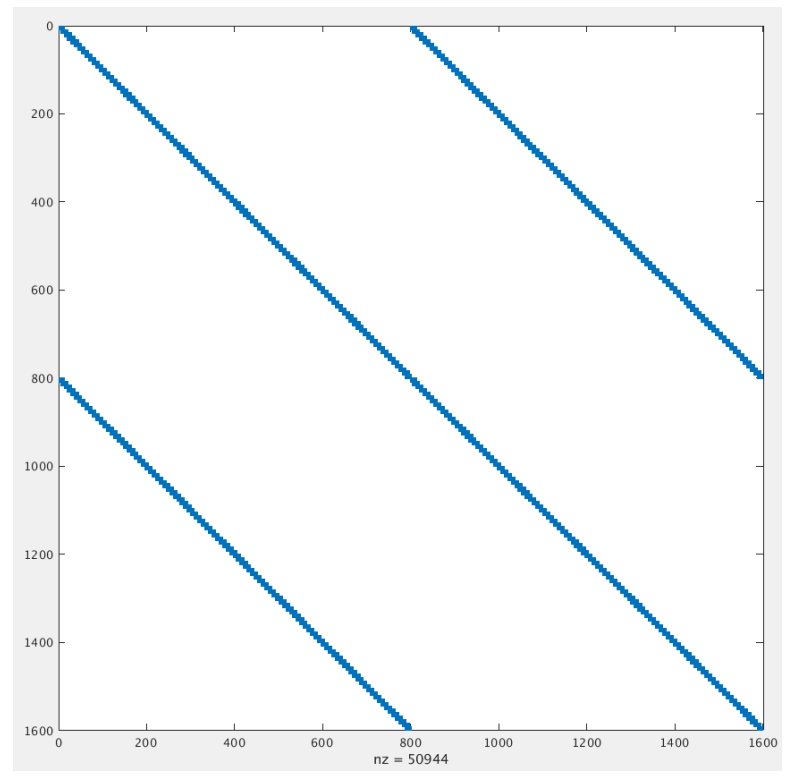

Figure 3.28: Matrix $A, 1 \mathrm{D}-2 \mathrm{EG}-S_{8}-100$ cells-upscattering.

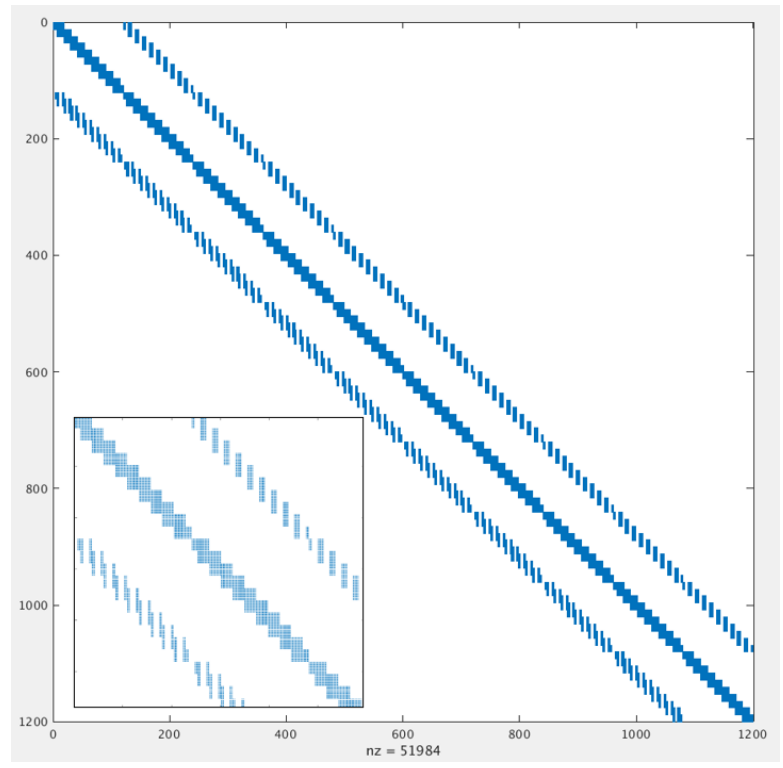

Figure 3.29: Matrix $A, 2 \mathrm{D}-1 \mathrm{EG}-S_{4}-10 \mathrm{x} 10$ cells. 


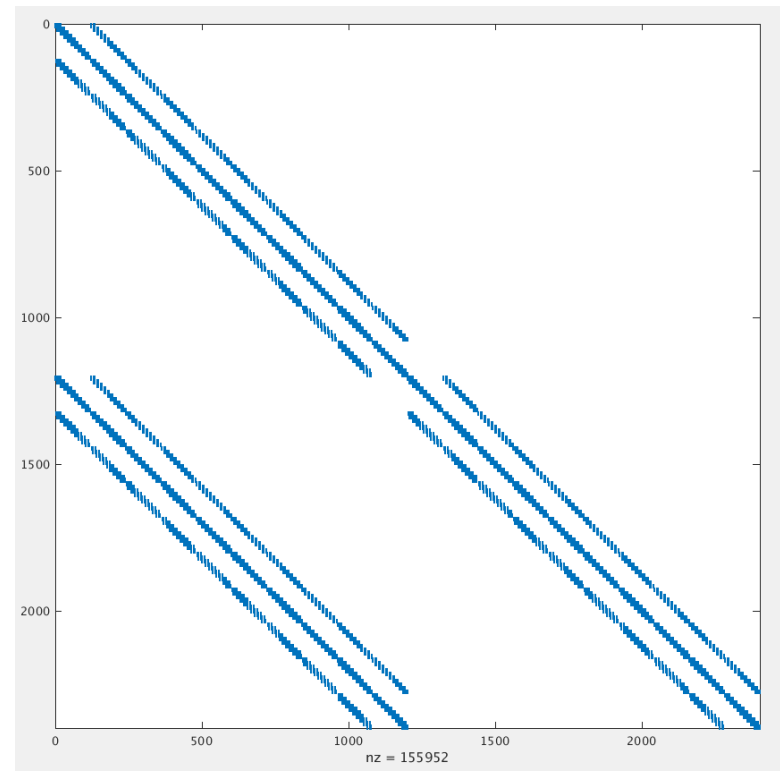

Figure 3.30: Matrix $A, 2 \mathrm{D}-2 \mathrm{EG}-S_{4}-10 \mathrm{x} 10$ cells-no upscattering.

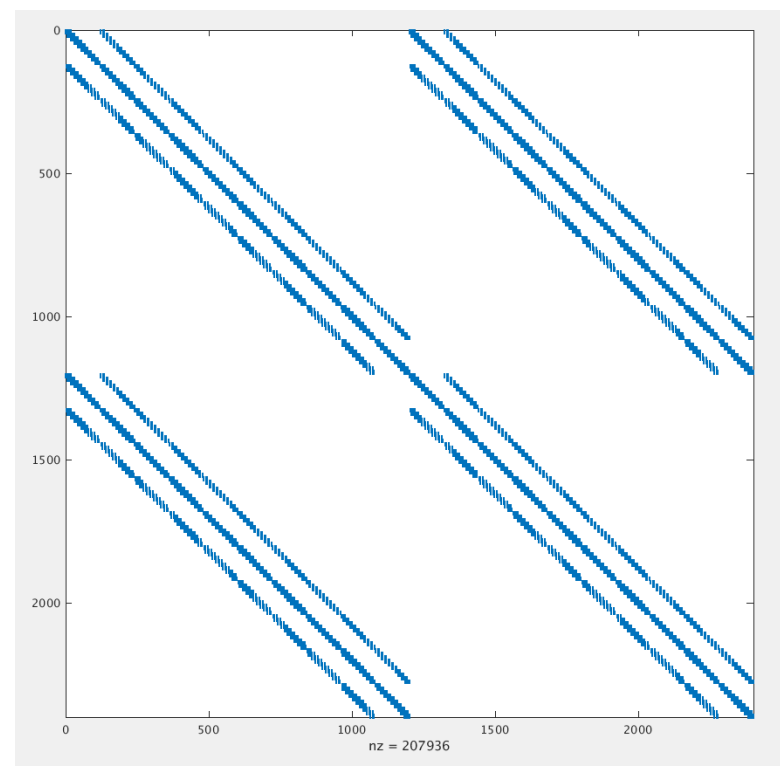

Figure 3.31: Matrix $A, 2 \mathrm{D}-2 \mathrm{EG}-S_{4}-10 x 10$ cells-upscattering. 


\subsection{Numerical Results for Criticality Problems}

The algorithms proposed in the previous sections were implemented in a FORTRAN program called n-DOTEC (neutron - Discrete Ordinates Transport Equation Code). This section shows $n$-DOTEC results for several one and two-dimensional problems or benchmarks. This section shows the results of eigenvalues and the neutron flux distribution or the power distribution. The results are evaluated by means of the eigenvalue errors, which are the relative errors multiplied by $10^{5}$ :

$$
\text { pcm }=\frac{\mid \text { ref.value }- \text { value } \mid}{\text { ref.value }} \cdot 1 \times 10^{5}
$$

Several 1D and four 2D benchmarks were selected to test the methods. The 1D cases carried out are: first the ISSA benchmark found in various works like [Issa et al., 1986], [Deo et al., 2014], [Talaat],the second is the seven region a heterogeneous slab problem with 7 different regions from [Kornreich and Parsons, 2004] and others anisotropic problems until 6 energy groups have been performed from Sood et al. [2003].

The chosen 2D cases are: first a MOX test problem found in [Capilla et al., 2008], the second one is a simple BWR cell test problem found in [Stepanek et al., 1982], [Kashi et al., 2017], the next one is the BWR rod bundle test also from [Kashi et al., 2017] and the last one is the common 2D C5G7 bechmark [Smith et al., 2003].

\subsubsection{One-dimensional cases}

To test the one-dimensional version of $n$-DOTEC, this section shows the results for several one-dimensional benchmark cases. The cases include different boundary conditions, both types of scattering (isotropic and anisotropic), different number of materials and different number of energy groups.

\section{ISSA Benchmark}

This is a one-energy one-dimensional slab problem with 2 regions, a fissile material on the left and a moderator material on the right (Fig.3.32). The material cross-sections are shown in Fig.3.32. The boundary condition on the left is reflective and the boundary condition on the right is vacuum. For the spatial discretization 100 mesh intervals were discretized. The Gauss-Legendre Quadrature was used with order $S_{8}$. The four largest eigenvalues obtained with n-DOTEC were 
$1.67835,0.798004$,

0.451980 , and 0.292597 . The reference value of the largest eigenvalue $\left(k_{e f f}\right)$ is 1.67840 for $S_{16}$ and 100 mesh intervals, calculated with ANISN (A One Dimensional Discrete Ordinates Transport Code With Anisotropic Scattering), a code supported by Oak Ridge National Laboratory [Issa et al., 1986]. The normalized scalar flux is displayed in Fig.3.33. As can be seen, the relative error is $2.979 \mathrm{pcm}$.

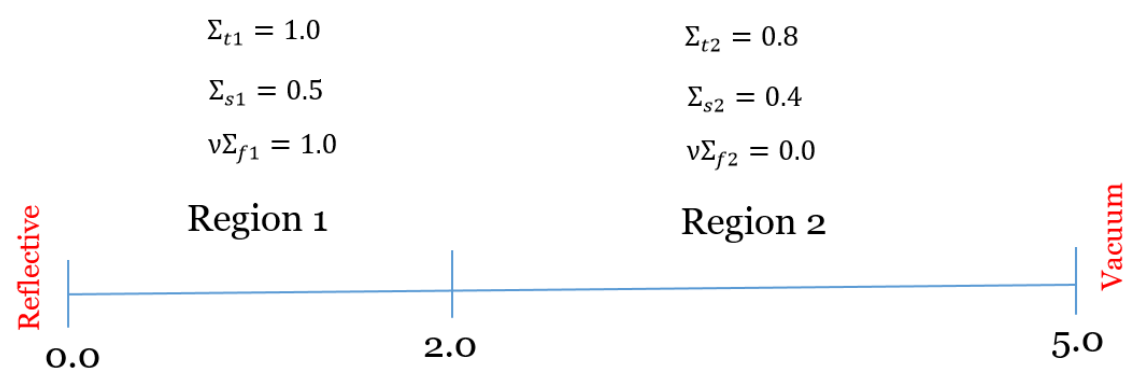

Figure 3.32: Geometry and cross sections for ISSA Benchmark.

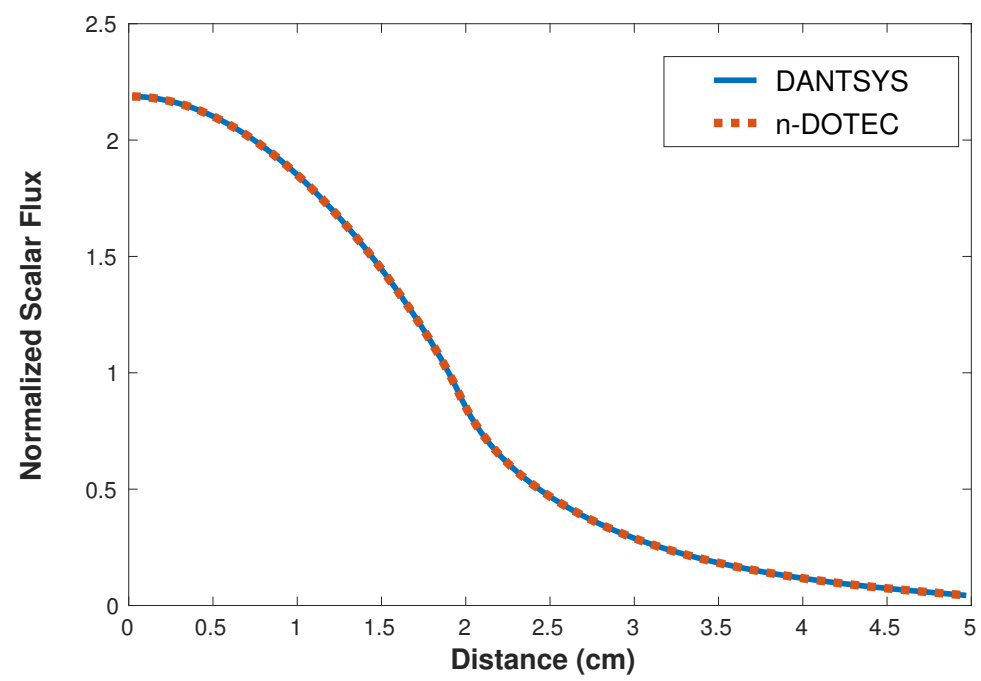

Figure 3.33: Normalized scalar flux for ISSA Benchmark calculated with $n-D O T E C$. 


\section{Seven Alternate Region}

This problem is composed of seven slab regions with three different combinations of fuel, reflector and absorber [Kornreich and Parsons, 2004]. The one-energy group cross sections for these materials are shown in Table 3.2. Three different cases are considered, one without absorber, one with absorber in position 5 and another with absorber in position 6. A scheme is shown in Fig.3.34. All cases use vacuum conditions for both boundaries. Each region is subdivided into 50 fine mesh cells. Gauss-Legendre quadrature set was selected with an order of $S_{32}$ for $n$-DOTEC simulation. Table 3.3 shows the results for the 3 largest eigenvalues. These results are compared with the reference values obtained by means of the Green's Function Method (GFM) [Kornreich and Parsons, 2004]. Also, the first eigenvalue was calculated by PARTISN with 500 fine mesh cells in each region and $S_{96}$. One can see that the values obtained by $n$-DOTEC are in agreement with the reference values. The eigenvalue error for the first eigenvalue in base case is $2.55 \mathrm{pcm}$, for the absorber in the position 5 is $141 \mathrm{pcm}$ and for the absorber in the position 6 is $15 \mathrm{pcm}$. Figure 3.35 shows a comparison between $n$-DOTEC results and PARTISN results.

Table 3.2: Cross-Sections for Seven Region Slab.

\begin{tabular}{lccc}
\hline & $\nu \Sigma_{f}\left(\mathrm{~cm}^{-1}\right)$ & $\Sigma_{s}\left(\mathrm{~cm}^{-1}\right)$ & $\Sigma_{t}\left(\mathrm{~cm}^{-1}\right)$ \\
\hline Fuel (U-235) & 0.178 & 0.334 & 0.415 \\
Reflector (Be) & 0.0 & 0.334 & 0.371 \\
Absorber (Be $w / c=0.1)$ & 0.0 & 0.037 & 0.371 \\
\hline
\end{tabular}

Base case

\begin{tabular}{l|l|l|l|l|l|l}
$R$ & Fuel & $R$ & Fuel & $R$ & Fuel & $R$
\end{tabular}

\begin{tabular}{l|l|l|l|l|l|l|l|} 
Abs. in Pos. 5 & $R$ & Fuel & $R$ & Fuel & Abs. & Fuel & $R$ \\
\hline Abs. in Pos. 6 & $R$ & Fuel & $R$ & Fuel & $R$ & Abs. & $R$ \\
\hline
\end{tabular}

Figure 3.34: Cases for Seven Region Slab. 


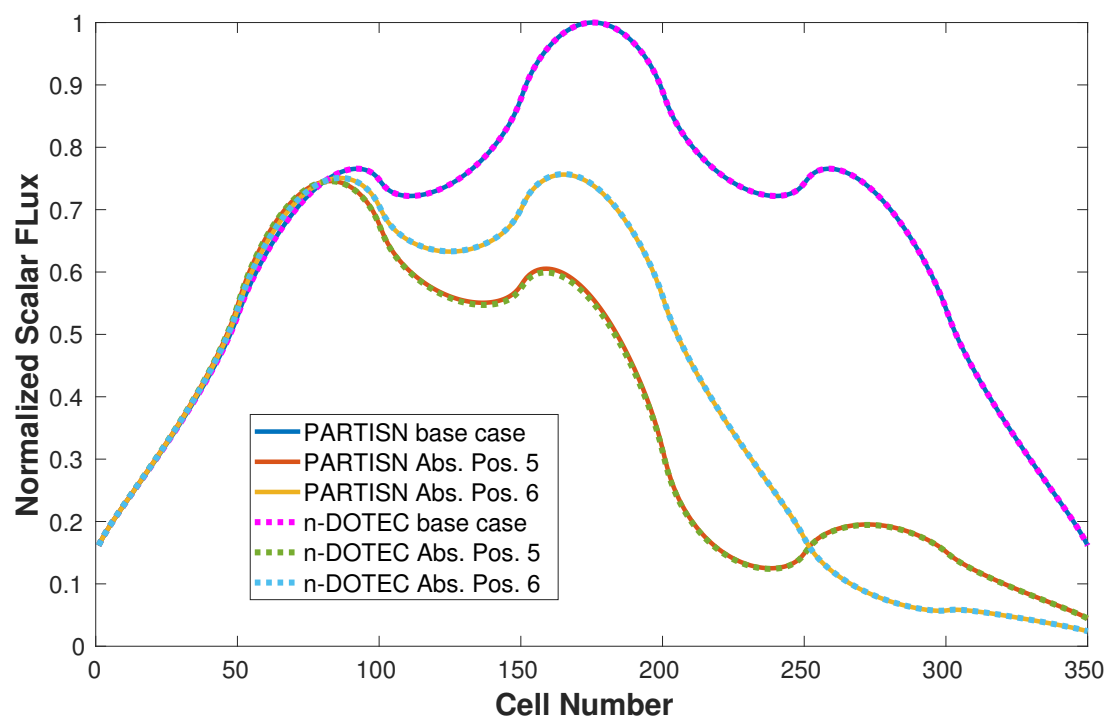

Figure 3.35: Scalar fluxes for each case of the 7 alternate region problem.

Table 3.3: Seven-region eigenvalues.

\begin{tabular}{lccccc}
\hline Case & $\mathrm{n}^{\circ}$ Eigenvalue & GFM & DANTSYS & $\mathrm{n}$-DOTEC & $\mathrm{pcm}\left(\Delta K_{\text {eff }}\right)$ \\
\hline No absorber & 1 & 1.17361 & 1.17361 & 1.17364 & 2.55 \\
& 2 & 0.758525 & - & 0.756857 & 219 \\
& 3 & 0.551768 & - & 0.549998 & 320 \\
\hline Abs. in Pos. 5 & 1 & 0.942676 & - & 0.941346 & 141 \\
& 2 & 0.655770 & - & 0.653801 & 300 \\
& 3 & 0.529032 & - & 0.527203 & 345 \\
\hline Abs. in Pos. 6 & 1 & 1.02265 & 1.02265 & 1.022493 & 15 \\
& 2 & 0.603382 & - & 0.601812 & 260 \\
& 3 & 0.208455 & - & 0.207234 & 585 \\
\hline
\end{tabular}

\section{Others validation problems}

For the validation of the code, in addition to the aforementioned cases, several additional cases with isotropic and anisotropic scattering has been modeled according to the Analytical Benchmark Test Set for Criticality Code Verification [Sood et al., 2003]. Table 3.4 shows a summary of the results of the multiplication factor for different problems included in the analytical benchmark, as well as the results for the previous cases. 
Table 3.4: Analytical Benchmark Results. $p c m=(\mid$ ref.value - value $\mid /$ ref.value $) \cdot 10^{5}$ $* A B=$ Analytical Benchmarck [Sood et al., 2003].

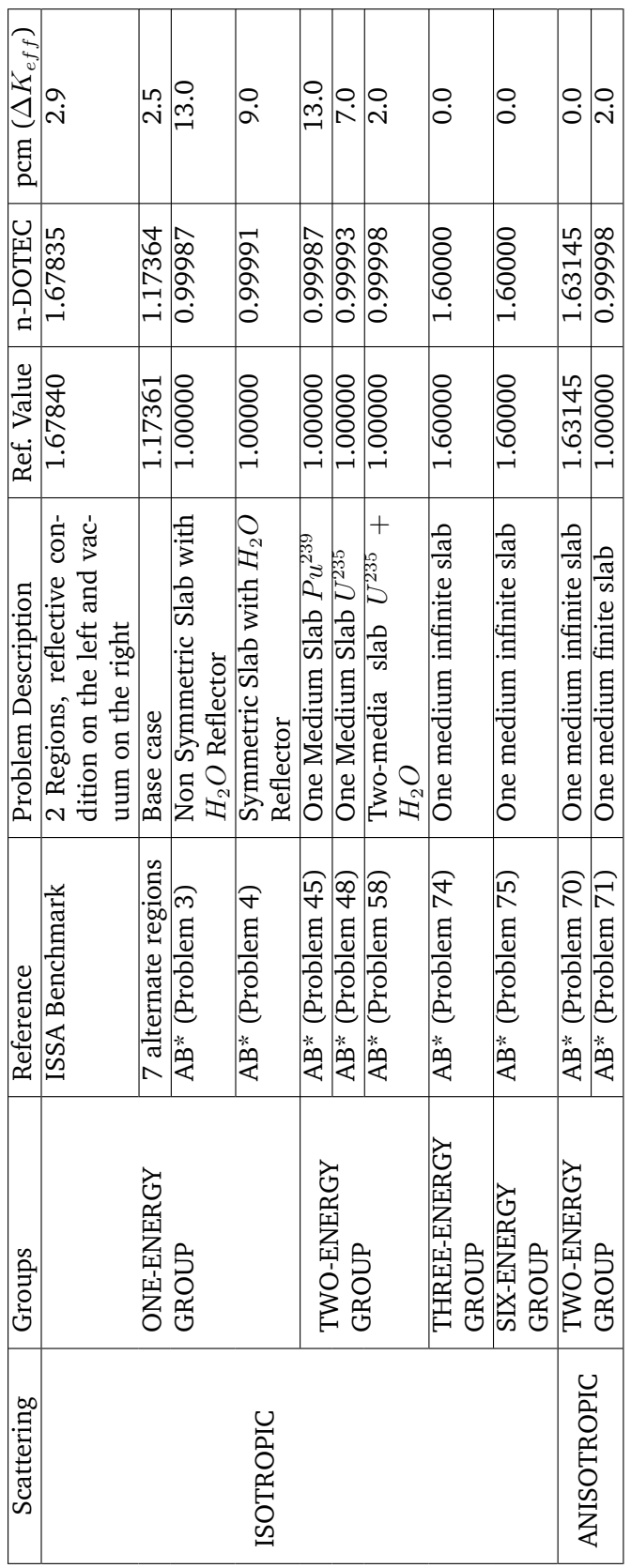




\subsubsection{Two-dimensional cases}

This section exhibits four cases to test the two-dimensional version of $n$-DOTEC program. The first one is the MOX test Problem, which was selected to check vacumm boundary conditions and non-homogeneous systems. Next two cases test reflective conditions in an homogeneous fuel and non-homogeneous fuel with rods: BWR cell test problem and BWR rod bundle test problem. Finally, the last problem is the C5G7 test problem, a known 2-D fuel assembly benchmark on deterministic transport without spatial homogenization.

\section{MOX test Problem}

This problem is a modification of the MOX benchmark problem described in [Capilla et al., 2008] and adapted from [Brantley and Larsen, 2000]. The core configuration is composed of $7 \times 7$ fuel assemblies of two types of fuel $\left(\mathrm{MOX} / \mathrm{UO}_{2}\right)$ as shown in Fig.3.36. The core is surrounded by a reflector material and each assembly has dimensions of $21.42 \mathrm{~cm} \times 21.42 \mathrm{~cm}$. This problem has two-energy groups and three different materials. The cross sections are shown in Table 3.5. The boundary conditions are vacuum.

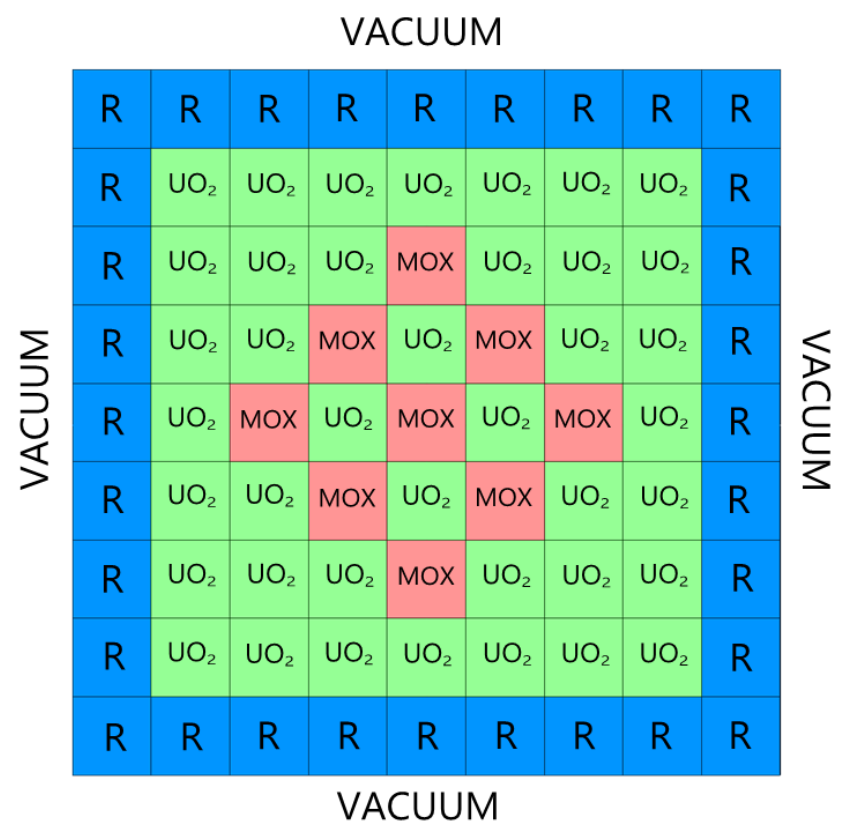

Figure 3.36: MOX benchmark problem geometry. 
The quadrature used in n-DOTEC was $S_{2}$ Gauss-Legendre Chebyshev $\left(P_{n}-T_{n}\right)$ quadrature set, the spatial discretization was a mesh of $36 \times 36$ elements. The four dominant eigenvalues calculated by n-DOTEC were compared with those calculated with the Spherical Harmonics Nodal Collocation (SHNC) in [Capilla et al., 2008]. SHNC code uses A Krylov-Schur method as eigensolver and linear systems were iteratively solved with the BCGSTAB method using HYPRE BoomerAMG as parallel preconditioner. The comparison is shown in Table 3.6. In Figs.3.37 and 3.38, the scalar flux distribution is displayed for the second and third subcritical degenerated modes. The results show good agreement with the reference values.

Table 3.5: MOX benchmark problem cross-sections. $g=1$ (fast energy group), $g=2$ (thermal energy group).

\begin{tabular}{ccccccc}
\hline Material & Group & $\Sigma_{t}$ & $\nu \Sigma_{f}$ & $\Sigma_{s, 1 \rightarrow g}$ & $\Sigma_{s, 2 \rightarrow g}$ & $\chi_{g}$ \\
\hline$M O X$ fuel & 1 & 0.550 & 0.0075 & 0.520 & 0.000 & 1.000 \\
& 2 & 1.060 & 0.450 & 0.015 & 0.760 & 0.000 \\
$U O_{2}$ fuel & 1 & 0.570 & 0.005 & 0.540 & 0.000 & 1.000 \\
& 2 & 1.100 & 0.125 & 0.020 & 1.000 & 0.000 \\
Reflector & 1 & 0.611 & 0.000 & 0.560 & 0.000 & 0.000 \\
& 2 & 2.340 & 0.000 & 0.050 & 2.300 & 0.000 \\
\hline
\end{tabular}

Table 3.6: Dominant eigenvalues for the MOX problem. SHNC=Spherical Harmonics Nodal Collocation.

\begin{tabular}{ccc}
\hline Eigenvalue & SHNC & $n$-DOTEC Leg-Cheby $S_{2}$ \\
\hline$K_{\text {eff }}$ & 0.9925 & 0.992538 \\
$2^{\text {nd }}$ eigen. & 0.9665 & 0.966344 \\
$3^{\text {rd }}$ eigen. & 0.9665 & 0.966344 \\
$4^{\text {th }}$ eigen. & 0.9399 & 0.939566 \\
\hline
\end{tabular}




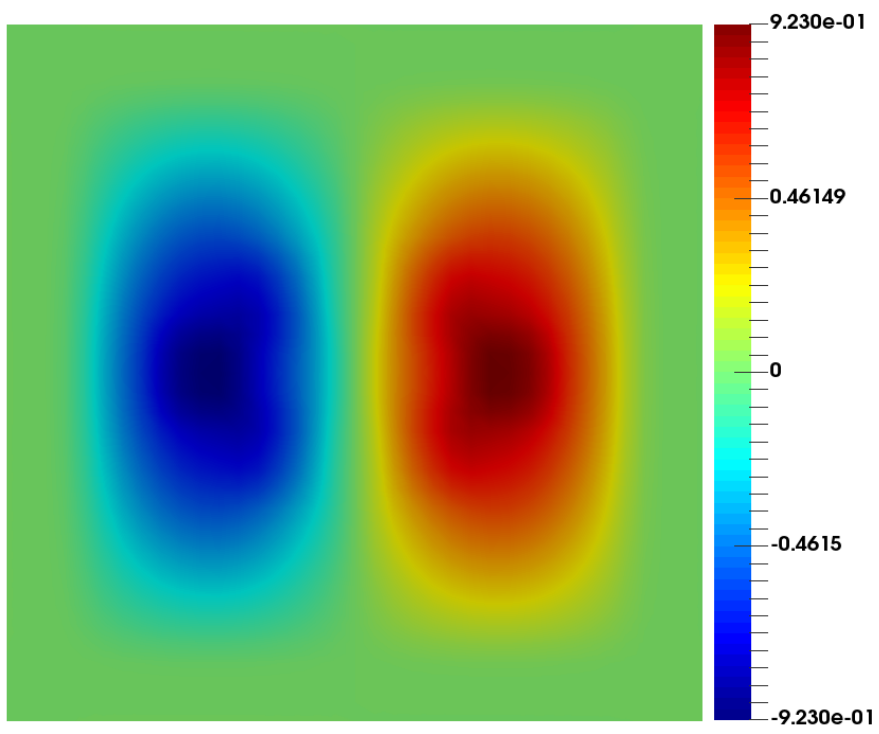

Figure 3.37: Scalar flux distribution for $2^{\text {nd }}$ eigenvalue.
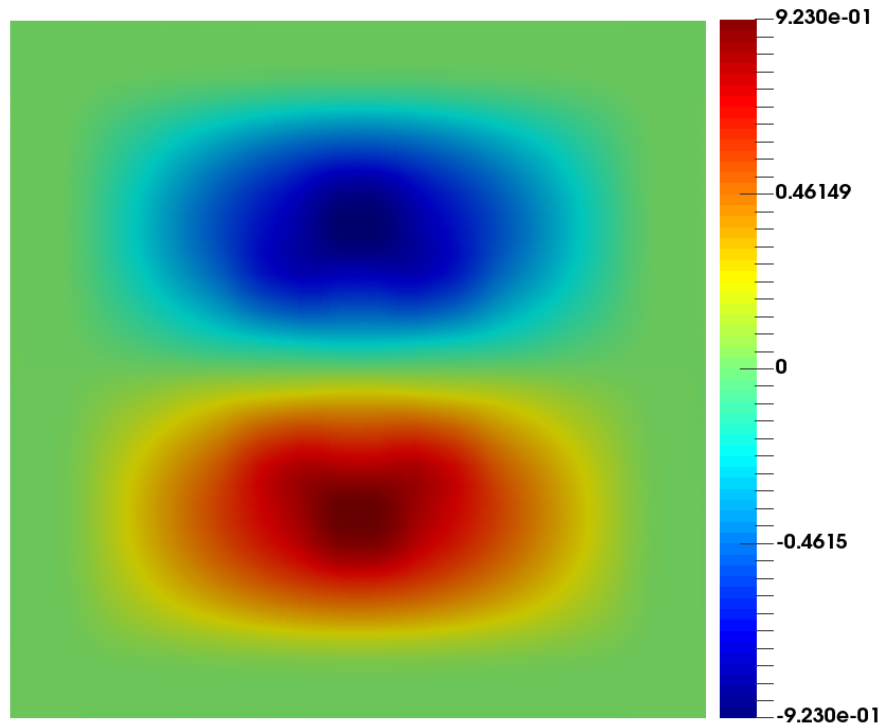

Figure 3.38: Scalar flux distribution for $3^{r d}$ eigenvalue. 


\section{BWR cell test problem}

The second case is a homogeneous BWR cell [Stepanek et al., 1982, Kashi et al., 2017]. This case has been selected due to the fact that it is modeled with upscattering. The problem is composed of a central homogenized fuel region surrounded by water moderator as shown in fig. 3.39. The two energy group crosssections are shown for the two materials of the problem in Table 3.7. All boundary conditions are reflective. The number of mesh intervals considered are $30 \times 30$ and the quadrature set is $P_{n}-T_{n}$ for different orders. The four largest eigenvalues were calculated, but the reference eigenvalue [Stepanek et al., 1982] calculated with the SURCU code developed at Federal Institute of Technology (Zurich, Switzerland) is 1.2127. In addition, the results calculated with DANTSYS were also compared. Table 5.9 shows n-DOTEC results for different orders of the quadrature sets. An study of the discretization influence has been performed keeping the order of the Discrete Ordinates set to $S_{8}$ for three different quadrature (Level-Symmetric, $P_{n}-E W$ and $P_{n}-T_{n}$ ), which can be seen in fig.3.40. This figure shows that the $k_{e f f}$ improves for large number of cells. The first group flux distribution for the four largest eigenvalues can be seen in fig.3.41. The second energy group is not showed due to the length of the thesis. The $k_{\text {eff }}$ calculated with $S_{8}$ has an error of $20 \mathrm{pcm}$ with respect to the reference and $0.083 \mathrm{pcm}$ with respect to DANTSYS with the same quadrature set and order. It can be appreciated that Table 3.8 shows practically same results for DANTSYS and n-DOTEC. This is due to the fact that the methodology developed in this work uses the same equations and similar discretization method. Furthermore, the same discretization of $30 \times 30$ is used in DANTSYS calculation than in n-DOTEC calculation.

Table 3.7: BWR cell benchmark problem cross-sections. $g=1$ (fast energy group), $g=2$ (thermal energy group).

\begin{tabular}{ccccccc}
\hline Material & Group & $\Sigma_{t}$ & $\nu \Sigma_{f}$ & $\Sigma_{s, 1 \rightarrow g}$ & $\Sigma_{s, 2 \rightarrow g}$ & $\chi_{g}$ \\
\hline Fuel & 1 & 0.196647 & 0.006203 & 0.178000 & 0.001089 & 1.000 \\
& 2 & 0.596159 & 0.1101 & 0.010020 & 0.525500 & 0.000 \\
Moderator & 1 & 0.222064 & 0.000 & 0.199500 & 0.001558 & 0.000 \\
& 2 & 0.887874 & 0.000 & 0.021880 & 0.878300 & 0.000 \\
\hline
\end{tabular}




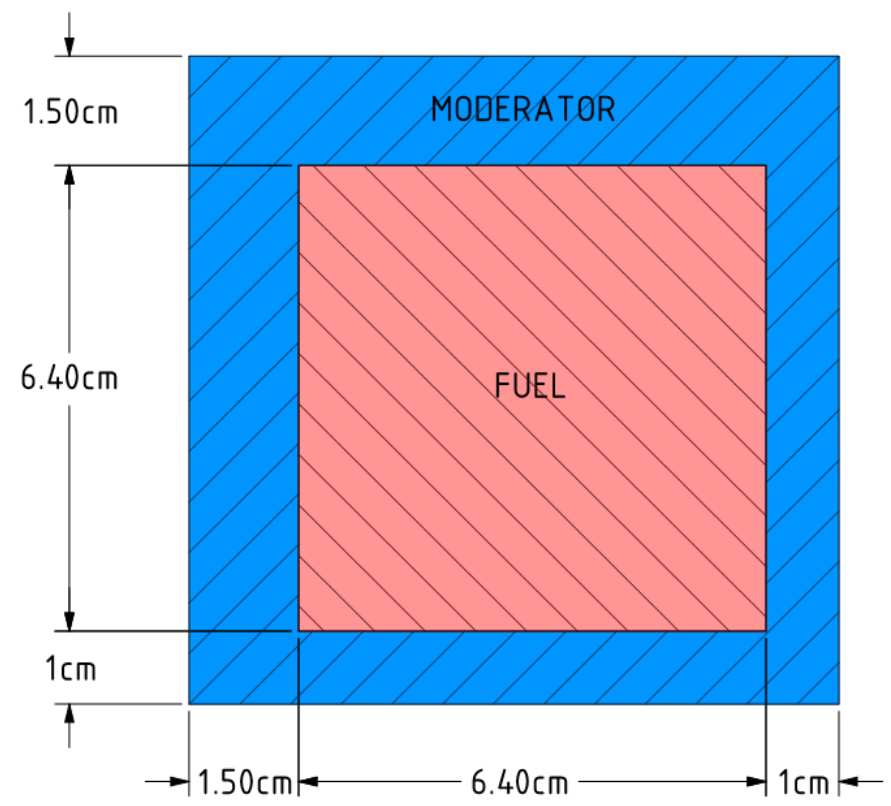

Figure 3.39: BWR cell problem geometry.

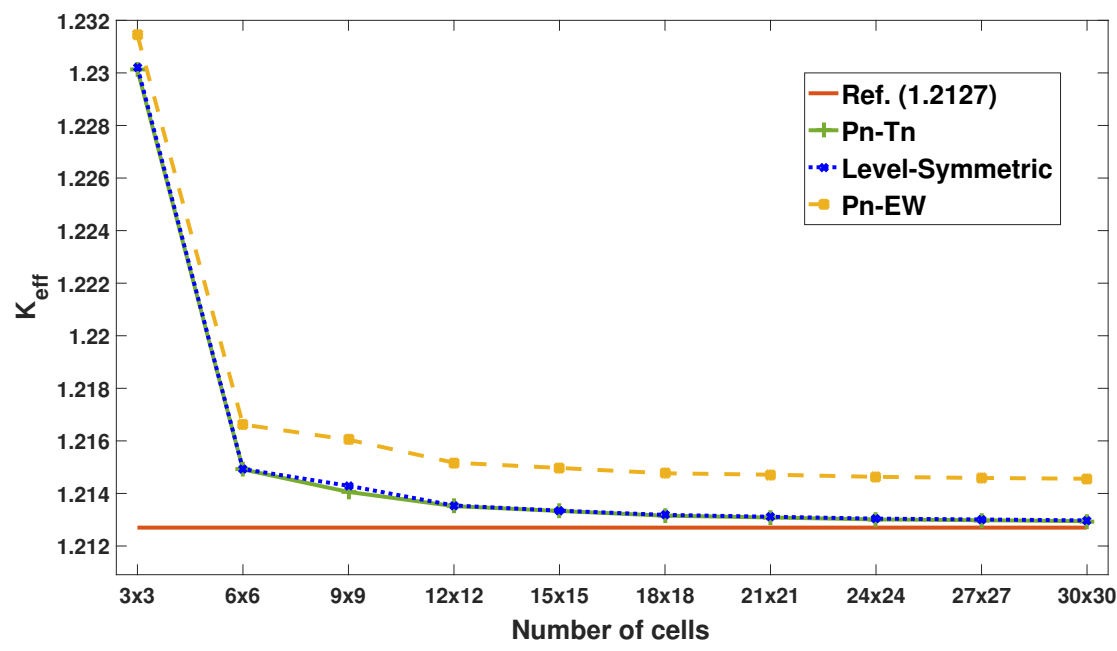

Figure 3.40: Spatial discretization influence. 

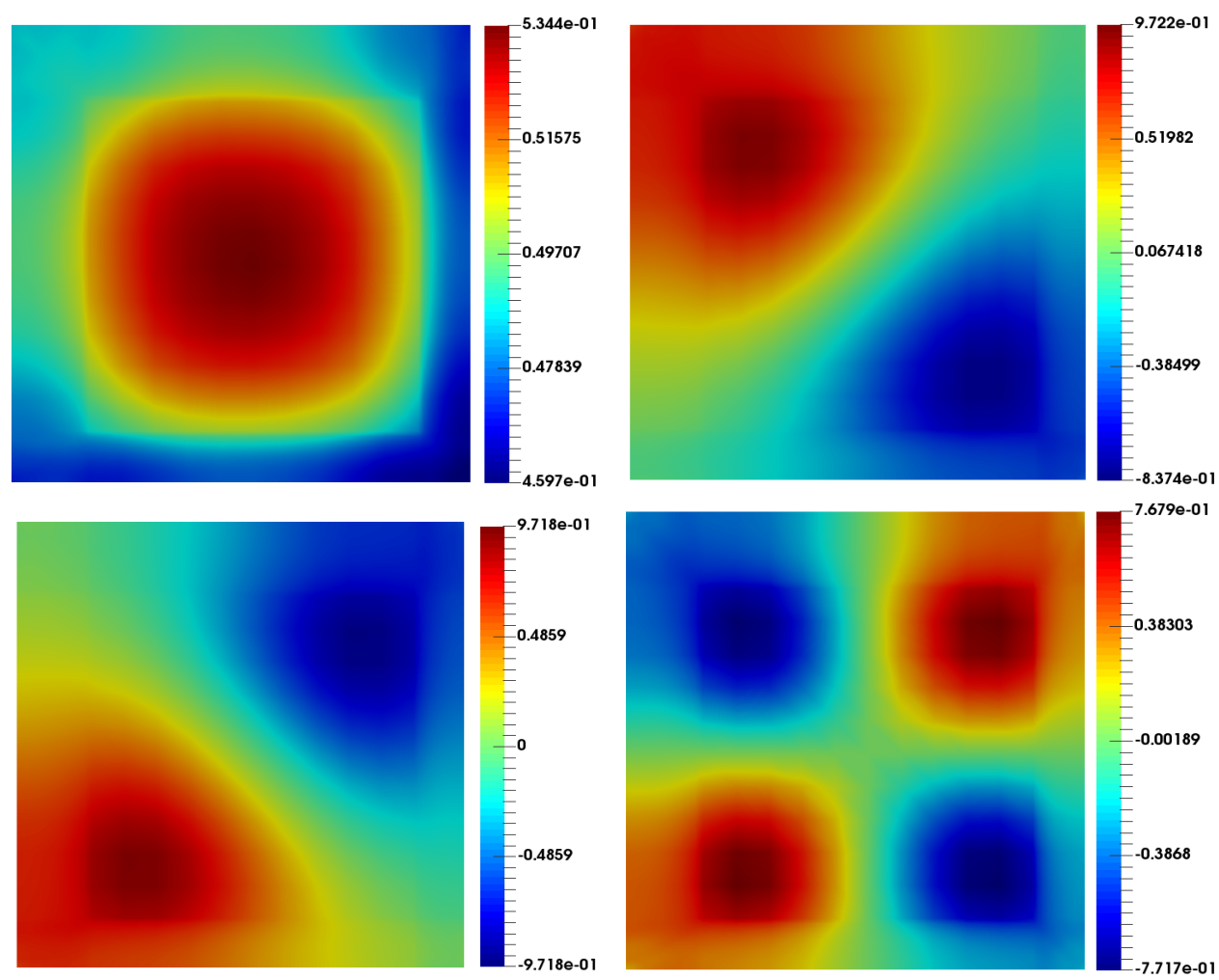

Figure 3.41: Four dominant eigenvalues flux distribution for the BWR cell test problem.

Table 3.8: BWR cell benchmark problem results for different quadrature order.

\begin{tabular}{cccccc}
\hline & Order & Quadrature & $\mathrm{n}^{\circ}$ of angular directions & $K_{\text {eff }}$ & $\mathrm{pcm}\left(\Delta K_{\text {eff }}\right)$ \\
\hline Reference value & - & - & - & 1.21270 & - \\
$n$-DOTEC & $S_{2}$ & Leg-Cheby & 4 & 1.21869 & 494 \\
DANTSYS & $S_{2}$ & Leg-Cheby & 4 & 1.21869 & 494 \\
$n$-DOTEC & $S_{4}$ & Leg-Cheby & 12 & 1.21427 & 129 \\
DANTSYS & $S_{4}$ & Leg-Cheby & 12 & 1.21427 & 129 \\
$n$-DOTEC & $S_{8}$ & Leg-Cheby & 40 & 1.21294 & 20 \\
DANTSYS & $S_{8}$ & Leg-Cheby & 40 & 1.21294 & 20 \\
\hline
\end{tabular}


$B W R$ rod bundle test problem

This test problem is a two-dimensional fuel bundle of a BWR [Kashi et al., 2017, Zolotar and Rahn, 1976]. There are seven materials in this case: materials ranging from 1 to 4 are different fuel types, material 5 is an homogenized fuel with poison. Surrounding these materials there is a wall assembly of stainless steel (material 6) which is surrounded by water (material 7). The problem has two energy groups and the cross-sections are displayed in Table 3.9. A scheme of this geometric configuration is shown in Fig.3.42. All boundary conditions are reflective. The $n$-DOTEC simulation used a spatial discretization of $1 \times 1$ for each cell of the problem and different Legendre-Chebyshev $\left(P_{n}-T_{n}\right)$ quadrature orders. The reference solution for this problem was calculated by using a mesh of $4 \times 4$ and angular approximation $S_{8}$ for the DOT code. Table 3.10 shows a comparison of the results. The eigenvalue error with respect to the reference is $49 \mathrm{pcm}$ for $S_{8}$ order and $7 \mathrm{pcm}$ with respect to DANTSYS. The first group flux distribution for the four dominant eigenvalues can be seen in fig.3.43.

Table 3.9: BWR rod bundle benchmark problem cross-sections.

\begin{tabular}{ccccccc}
\hline Material & Group & $\Sigma_{t}$ & $\nu \Sigma_{f}$ & $\Sigma_{s, 1 \rightarrow g}$ & $\Sigma_{s, 2 \rightarrow g}$ & $\chi_{g}$ \\
\hline 1 & 1 & 0.253100 & 0.0059250 & 0.2334270 & - & 1.000 \\
& 2 & 0.573200 & 0.0981700 & 0.010690 & 0.514280 & 0.000 \\
2 & 1 & 0.253600 & 0.0052420 & 0.2339200 & - & 1.000 \\
& 2 & 0.576700 & 0.0822800 & 0.010950 & 0.524960 & 0.000 \\
3 & 1 & 0.253500 & 0.0048200 & 0.2337900 & - & 1.000 \\
& 2 & 0.579070 & 0.0720000 & 0.011120 & 0.532530 & 0.000 \\
4 & 1 & 0.253300 & 0.0043370 & 0.2336900 & - & 1.000 \\
& 2 & 0.583700 & 0.0590000 & 0.011130 & 0.542300 & 0.000 \\
5 & 1 & 0.250600 & 0.0056050 & 0.2308400 & - & 1.000 \\
& 2 & 0.585300 & 0.0242400 & 0.010160 & 0.422700 & 0.000 \\
6 & 1 & 0.217200 & 0.0000000 & 0.2070700 & - & 0.000 \\
& 2 & 0.474800 & 0.0000000 & 0.009095 & 0.470416 & 0.000 \\
7 & 1 & 0.247600 & 0.0000000 & 0.2105800 & - & 0.000 \\
& 2 & 1.123000 & 0.0000000 & 0.036820 & 1.115200 & 0.000 \\
\hline
\end{tabular}




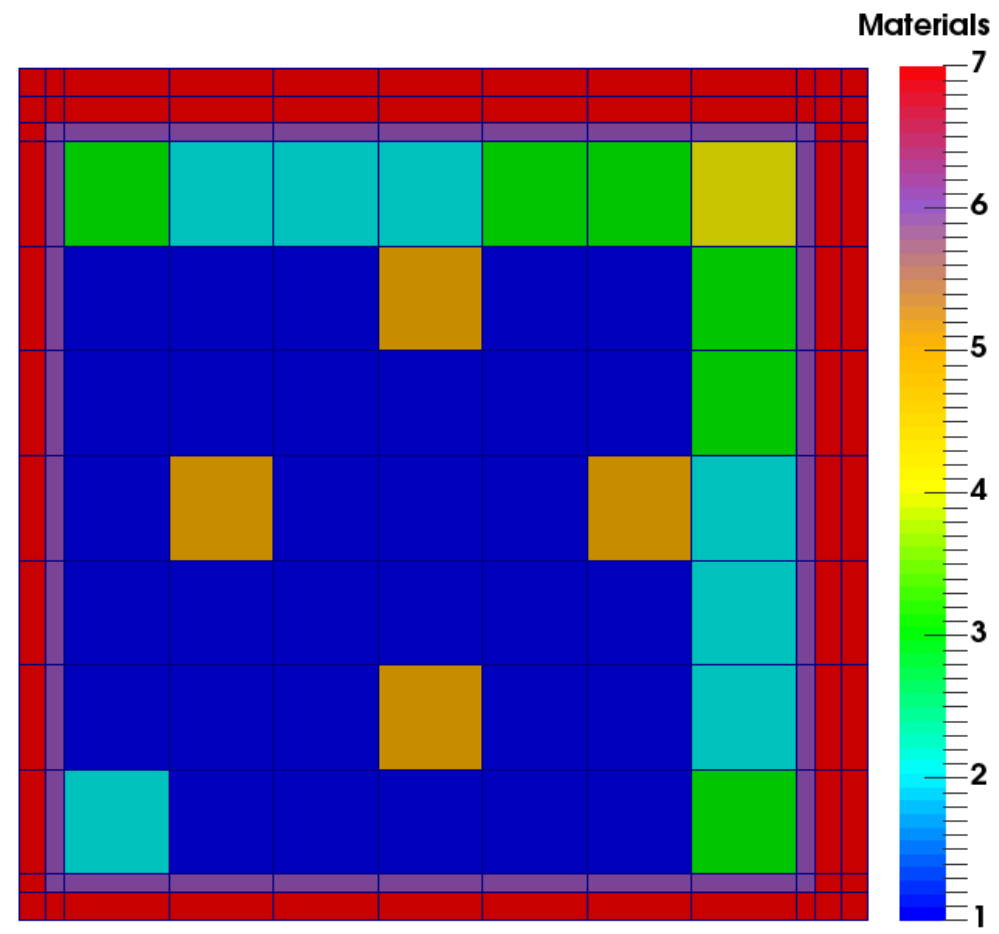

Figure 3.42: Material distribution and mesh for the BWR rod bundle test problem.

Table 3.10: BWR rod bundle benchmark problem multiplication factor results for different quadrature order.

\begin{tabular}{cccccc}
\hline & Order & Quadrature & $\mathrm{n}^{\circ}$ of angular directions & $K_{\text {eff }}$ & $\mathrm{pcm}\left(\Delta K_{\text {eff }}\right)$ \\
\hline Reference DOT & $S_{8}$ & - & 40 & 1.08714 & - \\
$n$-DOTEC & $S_{2}$ & Leg-Cheby & 4 & 1.09212 & 458 \\
DANTSYS & $S_{2}$ & Leg-Cheby & 4 & 1.09245 & 488 \\
$n$-DOTEC & $S_{4}$ & Leg-Cheby & 12 & 1.08871 & 145 \\
DANTSYS & $S_{4}$ & Leg-Cheby & 12 & 1.08881 & 154 \\
n-DOTEC & $S_{8}$ & Leg-Cheby & 40 & 1.08759 & 41 \\
DANTSYS & $S_{8}$ & Leg-Cheby & 40 & 1.08768 & 49 \\
\hline
\end{tabular}



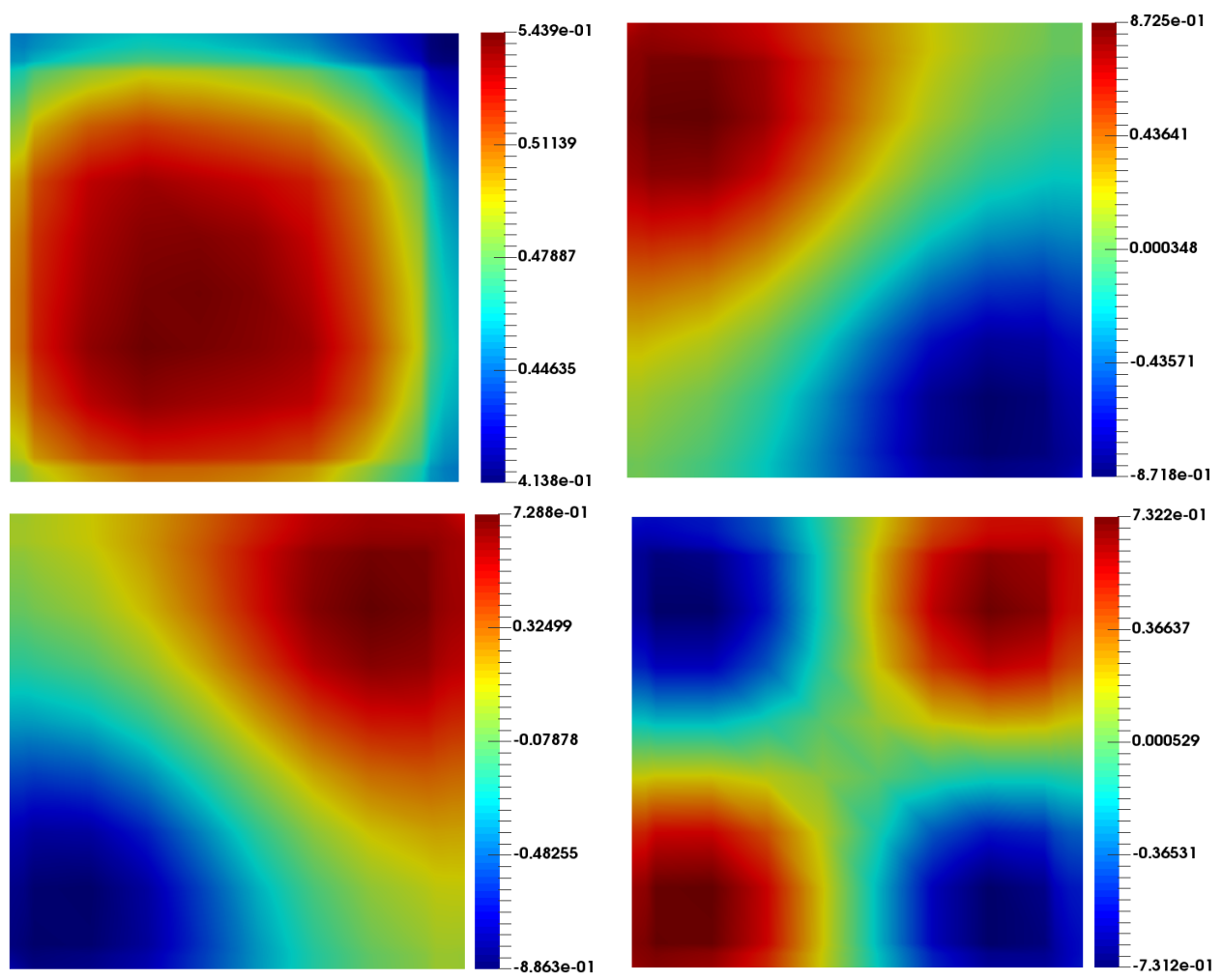

Figure 3.43: Four dominant eigenvalues flux distribution for the BWR rod bundle test problem.

\section{C5G7 test problem}

This case corresponds to a quarter symmetric core of the PWR C5G7 MOX fuel assembly problem [Smith et al., 2003]. The benchmark geometry is composed of 4 assemblies surrounded by a water reflector region, as can be seen in fig.3.44, as well as the boundary conditions. Each fuel assembly is made up of $17 \times 17$ square pitch array of cylindrical fuel pins. Since n-DOTEC can only deal with Cartesian geometry, these cylindrical pins are modeled by squares with the same area as the corresponding cylinders. A representation of this approximation is displayed in fig.3.45. The composition layout and the mesh is shown in fig.3.46. In addition, fig.3.47 displays a zoomed detail of fig.3.46. Cross-sections are described in the benchmark [Smith et al., 2003], with 7 energy groups for the seven corresponding materials. The reactor is composed of three MOX fuels with different enrichments, $\mathrm{UO}_{2}$ fuels, guide tubes, fission chambers and moderator. Table 
3.12 summarizes the comparison of the results obtained by $n$-DOTEC with $S 2$ and $S 4 P_{n}-T_{n}$ quadrature and those obtained by MCNP, which is the reference code. The $k_{e f f}$ error is $88 \mathrm{pcm}$ for $S_{4}$ order and the power mean relative error is $1.413 \%$. Other power comparison results show good agreement with respect to the benchmark results. Furthermore, the sub-critical modes have been compared in table 3.11, where it can be seen that the result is independent of the any kind of angular discretization [Capilla et al., 2018], because the modes are the same for the Spherical Harmonics method as for the Discrete ordinates method. Fig.3.48 shows the flux distribution for the energy groups 1 and 7 for the first eigenvalue, fig.3.49 shows the flux distribution for the energy groups 1 and 7 for the second eigenvalue and fig.3.50 shows the power distribution.

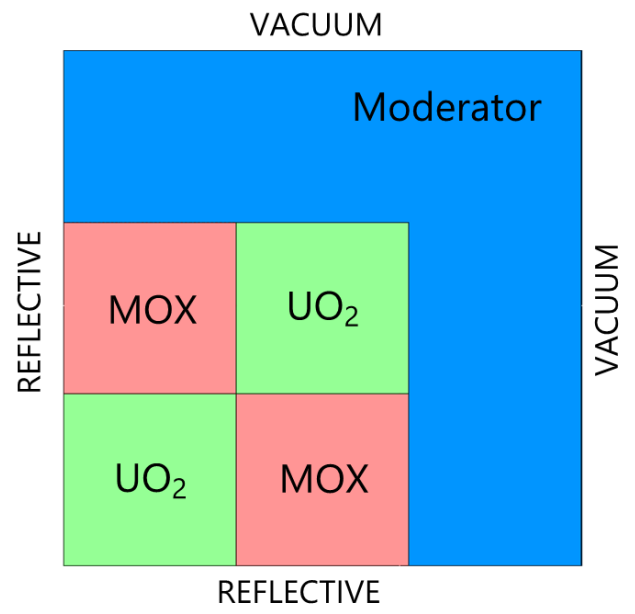

Figure 3.44: Assembly.
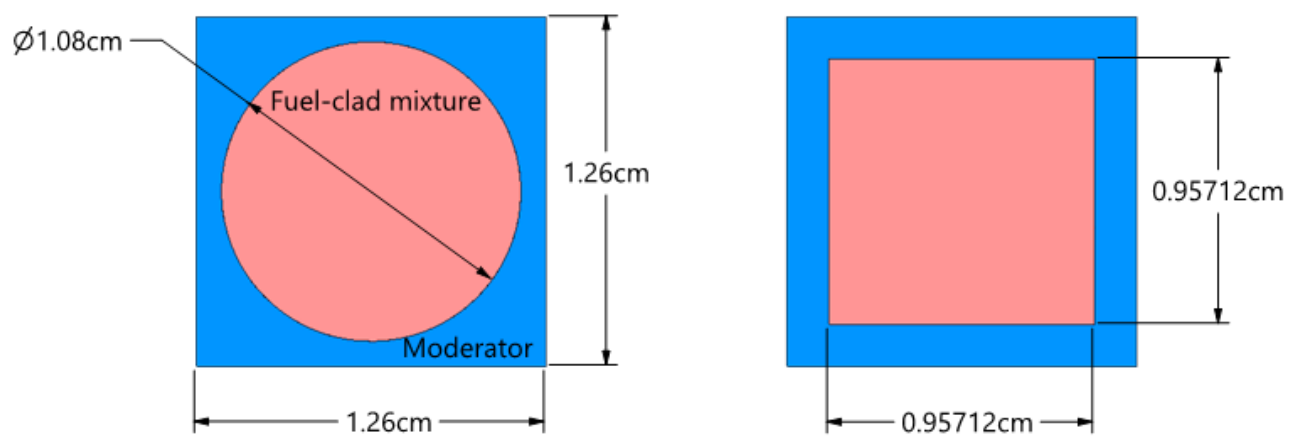

Figure 3.45: Pin cell approximation. 
Table 3.11: Sub-critical modes comparison. ${ }^{1}$ SHNC $=$ Spherical Harmonics Nodal Collocation.

\begin{tabular}{ccccc}
\hline mode & $n$-DOTEC S2 & $n$-DOTEC S4 & ${ }^{1}$ SHNC P1 & ${ }^{1}$ SHNC P3 \\
\hline 1st & 1.188877 & 1.187619 & 1.183847 & 1.177241 \\
2nd & 0.912220 & 0.918477 & 0.904490 & 0.910234 \\
3rd & 0.868536 & 0.873095 & 0.859548 & 0.867538 \\
4th & 0.730518 & 0.727383 & 0.703131 & 0.719696 \\
5th & 0.571266 & 0.592365 & 0.562243 & 0.587400 \\
6th & 0.570752 & 0.591601 & 0.561512 & 0.586667 \\
\hline
\end{tabular}

Materials

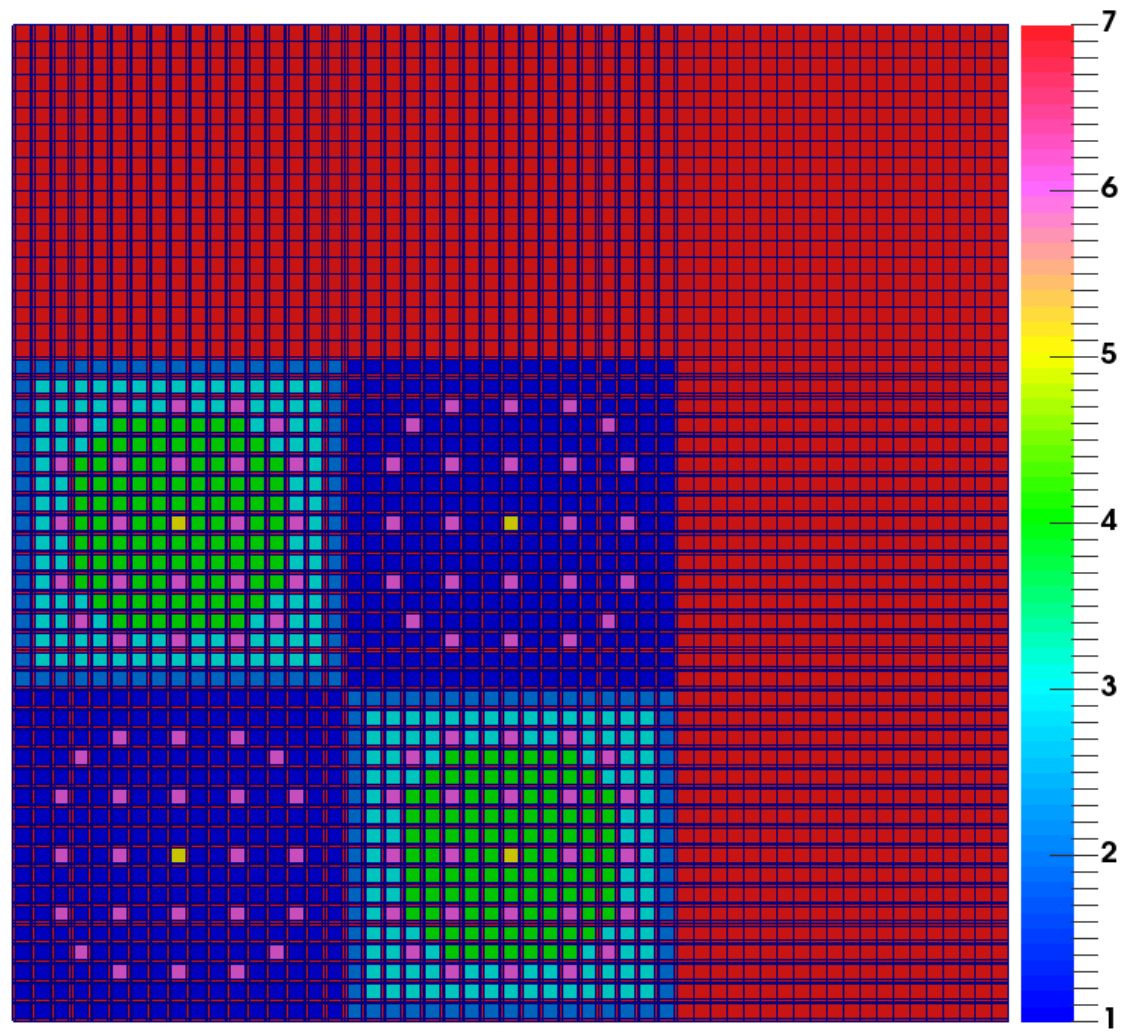

7

5 3

Figure 3.46: composition layout. 


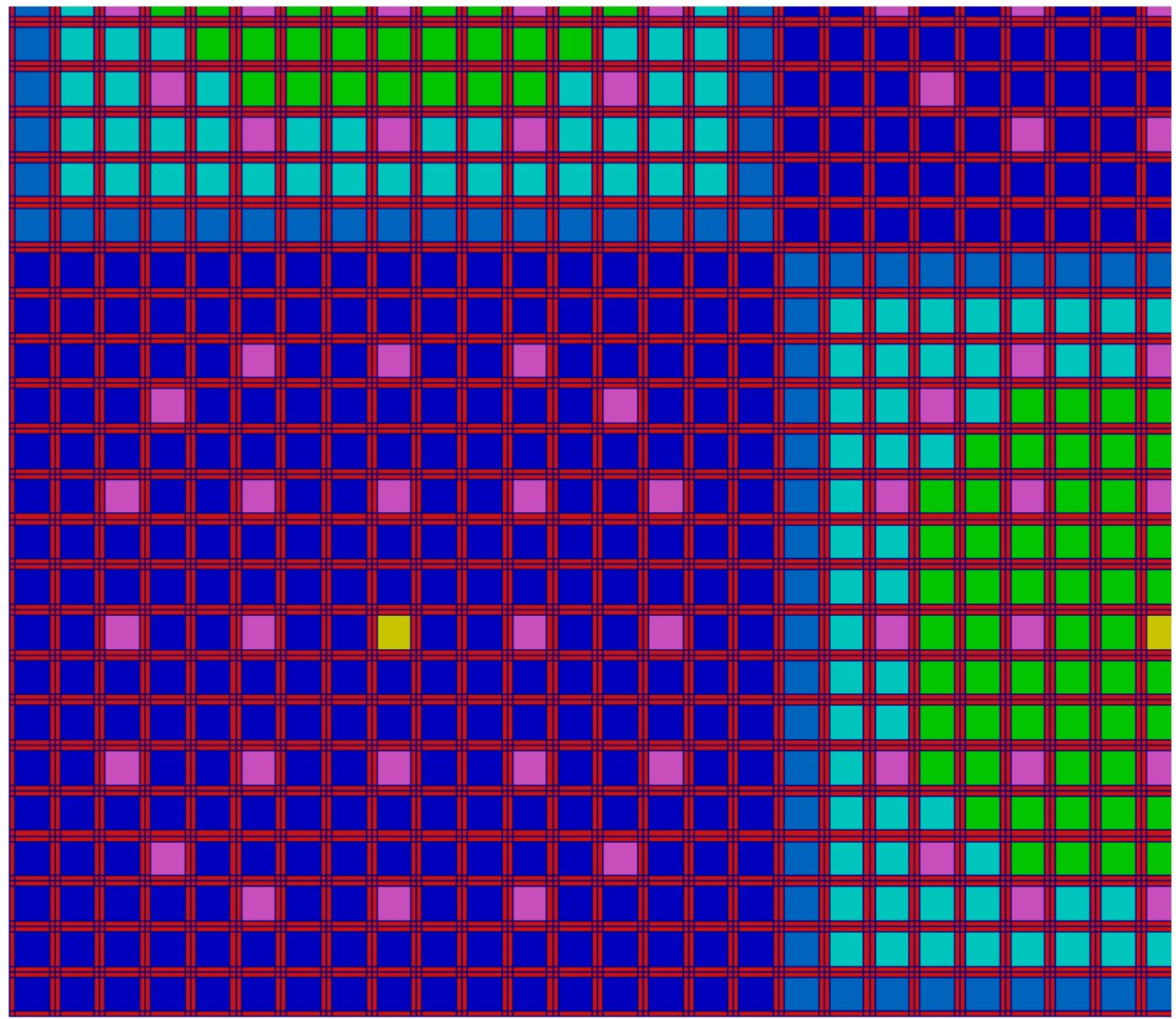

Figure 3.47: composition layout.
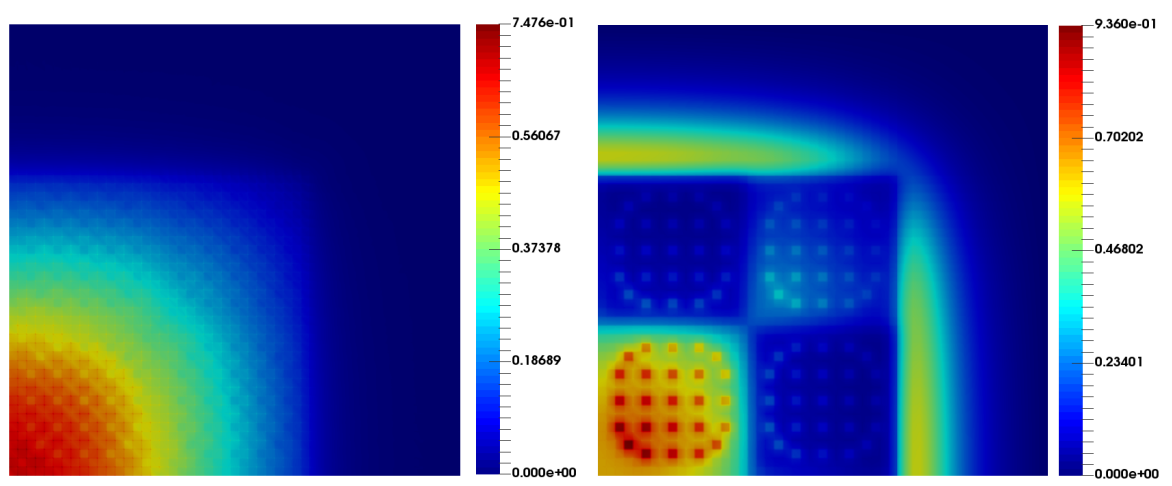

Figure 3.48: Flux of the first mode for energy groups 1 and 7. 


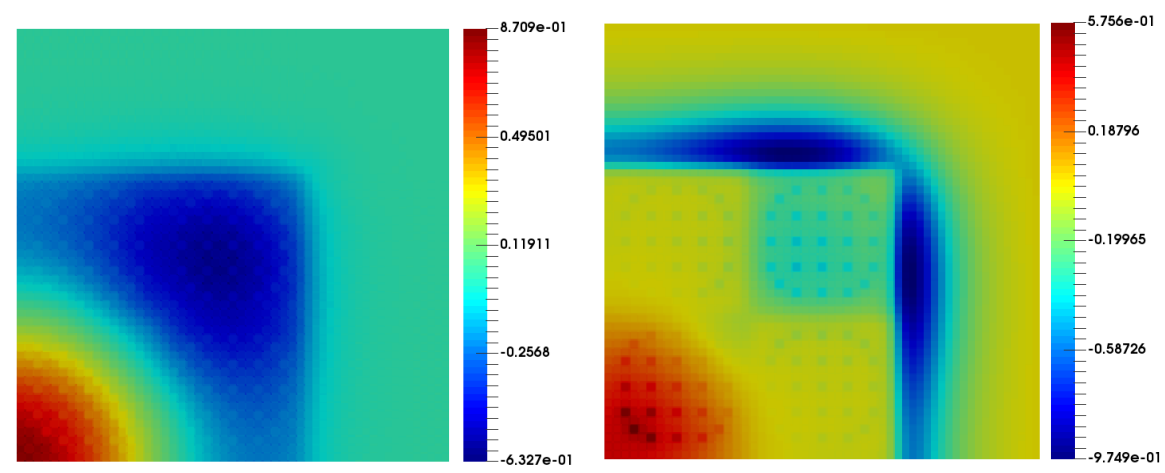

Figure 3.49: Flux of the second mode for energy groups 1 and 7.

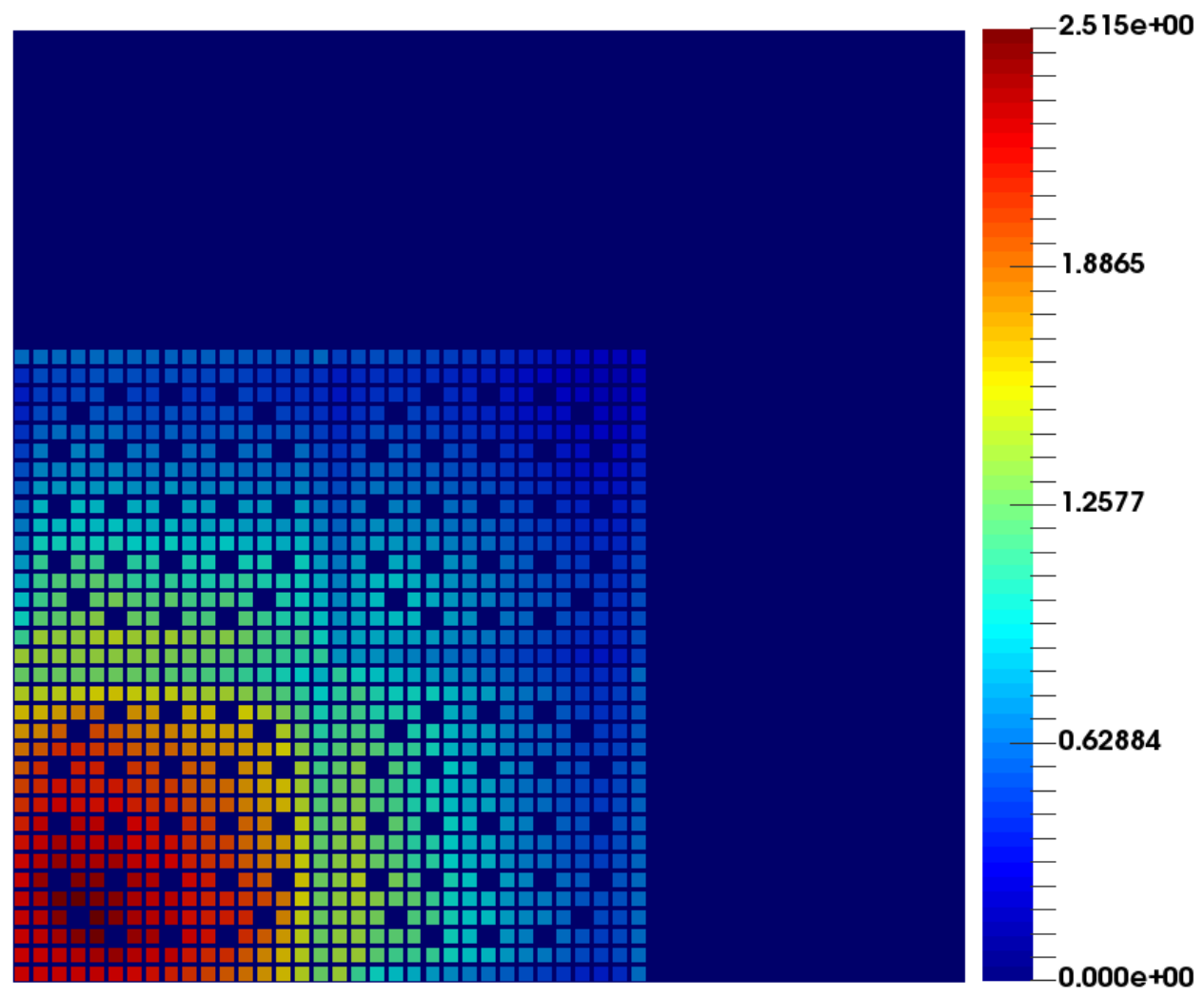

Figure 3.50: Power Distribution of the C5G7 problem. 
Table 3.12: C5G7 Test problem results.

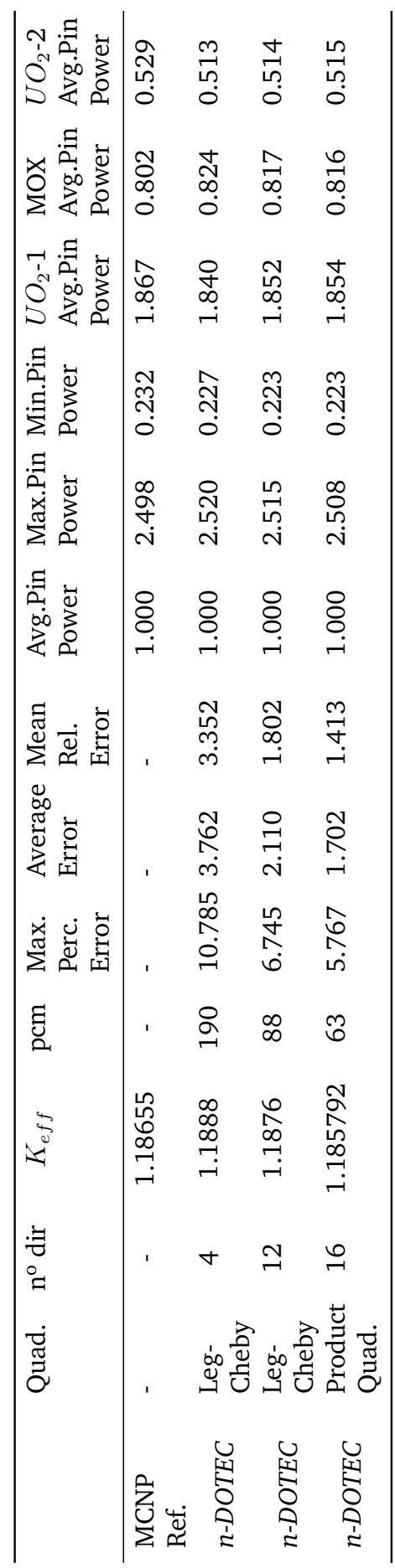




\subsection{Neutron Transport Fixed Source Problem}

Neutron fixed source problems systems consist in determining the neutron distribution on a medium from a known neutron source independent of the flux distribution.

Hence, the external source can be defined by the number of source particles emitted in $d V$ about $\vec{r}$, traveling in cone directions $d \hat{\Omega}$ about $\hat{\Omega}$ with energies between $E$ and $E+d E$, in the interval between $t$ and $t+d t$. The one-dimensional fixed source neutron transport equation for multiplying systems, can be expressed as:

$$
\begin{gathered}
\mu \frac{\partial \psi(x, \mu, E)}{\partial x}+\Sigma_{t}(x, E) \psi(x, \mu, E)= \\
\quad \int_{0}^{\infty} \int_{-1}^{1} \Sigma_{s}\left(x, \mu^{\prime}, \mu, E^{\prime} \rightarrow E\right) \psi\left(x, \mu^{\prime}, E^{\prime}\right) d \mu^{\prime} d E^{\prime} \\
+\chi(x, E) \int_{0}^{\infty} \int_{-1}^{1} \nu \Sigma_{f}\left(x, E^{\prime}\right) \psi\left(x, \mu^{\prime}, E^{\prime}\right) d \mu^{\prime} d E^{\prime}+S(x, \mu, E)
\end{gathered}
$$

In the conventional multi-group form of the former equation is:

$$
\begin{aligned}
\mu \frac{\partial}{\partial x} \psi_{g}(x, \mu)+\Sigma_{t, g}(x) \psi_{g}(x, \mu) & \\
= & \sum_{g^{\prime}=1}^{G} \int_{-1}^{1} \Sigma_{s, g^{\prime} \rightarrow g}\left(x, \mu^{\prime}, \mu\right) \psi_{g^{\prime}}\left(x, \mu^{\prime}\right) d \mu^{\prime} \\
& +\chi_{g}(x) \sum_{g^{\prime}=1}^{G} \int_{-1}^{1} \nu \Sigma_{f, g^{\prime}}(x) \psi_{g^{\prime}}\left(x, \mu^{\prime}\right) d \mu^{\prime}+S_{g}(x, \mu)
\end{aligned}
$$

And the discretized formulation using the Discrete Ordinates method $S_{N}$ and the Finite difference method is: 


$$
\begin{aligned}
& \mu_{n} \frac{\psi_{g}\left(i+1 / 2, \mu_{n}\right)-\psi_{g}\left(i-1 / 2, \mu_{n}\right)}{h}+\Sigma_{t, g}(i) \frac{\psi_{g}\left(i+1 / 2, \mu_{n}\right)+\psi_{g}\left(i-1 / 2, \mu_{n}\right)}{2} \\
- & \frac{1}{4} \sum_{g^{\prime}=1}^{G} \sum_{l=0}^{L} \Sigma_{s, g^{\prime} \rightarrow g, l}(i) P_{l}\left(\mu_{n}\right) \sum_{m=1}^{N} w_{m}\left[\psi_{g^{\prime}}\left(i+1 / 2, \mu_{m}\right)+\psi_{g^{\prime}}\left(i-1 / 2, \mu_{m}\right)\right] P_{l}\left(\mu_{m}\right) \\
= & \frac{\chi_{g}(i)}{4} \sum_{g^{\prime}=1}^{G} \nu_{g^{\prime}} \Sigma_{f, g^{\prime}}(i) \sum_{m=1}^{N} w_{m}\left[\psi_{g^{\prime}}\left(i+1 / 2, \mu_{m}\right)+\psi_{g}\left(i-1 / 2, \mu_{m}\right)\right]+S_{g}\left(i, \mu_{n}\right)
\end{aligned}
$$

Where:

$$
\begin{aligned}
& 1 \leq n \leq N \quad \text { with } N \text { the number of discrete directions } \\
& 1 \leq g \leq G \quad \text { with } G \text { the total number of energy groups }
\end{aligned}
$$

$0 \leq l \leq L$ with $L$ the order of the Legendre polynomial scattering expansion $w_{m}$ are the weighting coefficients of directions $\mu_{m}$ and $0 \leq i \leq I$ is the mesh cell number

Similarly to one-dimensional case, the two dimensional steady-state multi-group neutron transport equation discretized with the discrete ordinates method takes the form:

$$
\begin{gathered}
\mu_{n} \frac{\partial \psi_{g}\left(x, y, \mu_{n}, \eta_{n}\right)}{\partial x}+\eta_{n} \frac{\partial \psi_{g}\left(x, y, \mu_{n}, \eta_{n}\right)}{\partial y}+\Sigma_{t, g}(x, y, E) \psi_{g}\left(x, y, \mu_{n}, \eta_{n}\right)= \\
q_{s}(x, y)+\frac{\chi_{g}(x, y)}{8} \sum_{g^{\prime}=1}^{G} \nu_{g^{\prime}}(x, y) \Sigma_{f, g^{\prime}}(x, y) \sum_{m=1}^{N} w_{m} \psi_{g^{\prime}}\left(x, y, \mu_{m}, \eta_{m}\right)+S_{g}\left(x, y, \mu_{n}, \eta_{n}\right)
\end{gathered}
$$

As mentioned before the $q_{s}(x, y)$ term is expressed as:

$$
\begin{gathered}
q_{s}(x, y)=\frac{1}{8} \sum_{g^{\prime}=1}^{G} \sum_{l=0}^{L}(2 l+1) \Sigma_{s, g^{\prime} \rightarrow g, l}(x, y)\left\{P_{l}\left(\mu_{n}\right) \sum_{m=1}^{N} w_{m} P_{l}\left(\mu_{m}\right) \psi_{g^{\prime}}\left(x, y, \mu_{m} \eta_{m}\right)\right. \\
+2 \sum_{k=1}^{l} \frac{(l-k) !}{(l+k) !} P_{l}^{k}\left(\mu_{n}\right)\left[\sum_{m=1}^{N} w_{m} P_{l}^{k}\left(\mu_{m}\right) \psi_{g^{\prime}}\left(x, y, \mu_{m}, \eta_{m}\right) \cos \left(k \varphi_{m}\right) \cos \left(k \varphi_{n}\right)\right. \\
\left.\left.+\sum_{m=1}^{N} w_{m} P_{l}^{k}\left(\mu_{m}\right) \psi_{g^{\prime}}\left(x, y, \mu_{m}, \eta_{m}\right) \sin \left(k \varphi_{m}\right) \sin \left(k \varphi_{n}\right)\right]\right\}
\end{gathered}
$$


To simplify the notation of the two-dimensional equation, the FDM method for this equation is applied separately as in the eq.3.109, 3.110 and 3.111.

However, physically there are not many problems in which exist at the same time fission sources and fixed sources. Medical field for example, only presents problems in which is very common the fixed source but not the fission source. These kind of problems are radiotherapy treatment planifications, brachytherapy, and radiation protection, shielding, etc.; where could be used for calculating the dose absorbed for the patient. Another example could be the calculation dose per organ in computed tomography image acquisitions. Radiotherapy treatment devices use high energy particle beams between 6 and $15 \mathrm{MeV}$, most part of time this particles are photons and in some cases neutrons [Morató et al., 2019]. Both kind of particles can be simulated by the transport equation, with the only difference of the cross sections. In these cases, the energy of the fixed source particles is no unique, is an X-Ray or neutron spectrum [Juste et al., 2020b, Morató et al., 2017]. On the other hand, bachytherapy uses radioactive seeds or liquid as ${ }^{125} I$ seeds or ${ }^{177} \mathrm{Lu}$-PSMA where the energy of the particles are discrete values and it is important the activity decay of the source material [Juste et al., 2020a, Morató et al., 2018].

A simpler formulation of the fixed source problem in a non-multiplying system can be written for one-dimensional and two-dimensional case:

$$
\begin{array}{r}
\mu_{n} \frac{\psi_{g}\left(i+1 / 2, \mu_{n}\right)-\psi_{g}\left(i-1 / 2, \mu_{n}\right)}{h}+\Sigma_{t, g}(i) \frac{\psi_{g}\left(i+1 / 2, \mu_{n}\right)+\psi_{g}\left(i-1 / 2, \mu_{n}\right)}{2} \\
-\frac{1}{4} \sum_{g^{\prime}=1}^{G} \sum_{l=0}^{L} \Sigma_{s, g^{\prime} \rightarrow g, l}(i) P_{l}\left(\mu_{n}\right) \sum_{m=1}^{N} w_{m}\left[\psi_{g^{\prime}}\left(i+1 / 2, \mu_{m}\right)+\psi_{g^{\prime}}\left(i-1 / 2, \mu_{m}\right)\right] P_{l}\left(\mu_{m}\right) \\
\left.=S_{g}\left(i, \mu_{n}\right)\right]
\end{array}
$$

The set of eqs.3.133 and 3.134, for 1D and 2D respectively can be expressed in matrix form as:

$$
A \psi=S
$$


where $A$ is a matrix with size $[N \times G \times I] \times[N \times G \times I]$ for 1D or with size $[N \times G \times I \times J] \times[N \times G \times I \times J]$ for 2D case, that includes the leakage term, and scattering term. $S$ is a vector with size $[N \times G \times I]$ for 1D case or $[N \times G \times I \times J]$ for $2 \mathrm{D}$ case, that is the independent term associated with the source term in units of $n / \mathrm{cm}^{2} s$.

The obtained linear system is solved using the LU factorization from the computational library MUMPS (MUltifrontal Massively Parallel sparse direct Solver) [Amestoy et al., 1998].

\subsection{Numerical Results for Fixed Source Problems}

The methodology explained in previous section has been also implemented into the FORTRAN code called n-DOTEC (neutron - Discrete Ordinates Transport Equation Code). The code solves isotropic and anisotropic fixed source problems for any $S_{n}$ approximation [Capilla et al., 2016]. Some calculations are presented in these sections to prove the accuracy of the method. Two error magnitudes are defined to compare the results calculated with n-DOTEC with reference values, the relative error $E_{r}$ and the maximum relative error $E_{\text {max }}$ :

$$
E_{r}=\frac{\left|\phi_{i}-\phi_{i}^{r e f}\right|}{\phi_{i}^{r e f}}, \quad E_{\max }=\max \left(E_{r}\right)
$$

\subsubsection{One-dimensional source problem with vacuum boundary conditions}

This is an academical test problem consisting of a one-group simple slab model of length $l_{x}=1 \mathrm{~cm}$ with isotropic homogeneous fixed source without scattering and fission terms. The boundary conditions are vacuum at both outer boundaries. The total cross-section is $\Sigma_{t}=1 \mathrm{~cm}^{-1}$ and the neutron source strength is $S=$ $1 \mathrm{n} / \mathrm{cm}^{2} \mathrm{~s}$. Different spatial nodes has been considered to test the method 10, 100 and 1000 nodes. The reference results are the analytical values calculated by numerical integration using the code Quadpack [Piessens and De DonckerKapenga]. The scalar fluxes are given in Tables 3.13 and 3.14 for different $S_{N}$ approximations. Tables 3.13 and 3.14 also show the relative error between the reference values and the different $S_{N}$ approximations. It is clearly seen that when the spatial or angular discretization is finer, the flux value is closer to the reference value. 


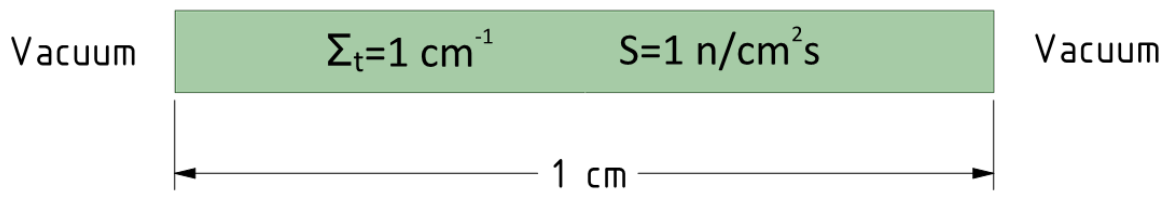

Figure 3.51: One-dimensional source problem with vacuum boundary conditions.

Table 3.13: Scalar fluxes for the homogeneous slab with vacuum boundary conditions and 10 spatial cells for different $S_{N}$.

\begin{tabular}{lccccccc}
\hline$x(\mathrm{~cm})$ & Ref. & $S_{2}$ & $S_{4}$ & $S_{8}$ & $S_{16}$ & $S_{32}$ & $S_{64}$ \\
\hline 0 & 0.42575 & 0.41192 & 0.42876 & 0.42657 & 0.42601 & 0.42605 & 0.42605 \\
0.1 & 0.55252 & 0.47492 & 0.51895 & 0.53806 & 0.55216 & 0.56087 & 0.56464 \\
0.2 & 0.61247 & 0.52204 & 0.58277 & 0.60729 & 0.61580 & 0.61521 & 0.61196 \\
0.3 & 0.64797 & 0.55472 & 0.62516 & 0.64898 & 0.65107 & 0.64971 & 0.65081 \\
0.4 & 0.66722 & 0.57395 & 0.64937 & 0.67130 & 0.66962 & 0.66872 & 0.66842 \\
0.5 & 0.67336 & 0.58029 & 0.65724 & 0.67832 & 0.67546 & 0.67480 & 0.67510 \\
\hline$E_{\max }(\%)$ & & 14.763 & 6.075 & 2.617 & 0.544 & 1.513 & 2.194 \\
\hline$E_{r}(\%)$ & & 12.374 & 3.370 & 0.860 & 0.304 & 0.457 & 0.538 \\
\hline
\end{tabular}

Table 3.14: Scalar fluxes for the homogeneous slab with vacuum boundary conditions and 100 spatial cells for different $S_{N}$. (* corresponds to calculation with 1000 spatial cells).

\begin{tabular}{lcccccccc}
\hline $\mathrm{x}(\mathrm{cm})$ & Ref. & $S_{2}$ & $S_{4}$ & $S_{8}$ & $S_{16}$ & $S_{32}$ & $S_{64}$ & $* 1000 / S_{32}$ \\
\hline 0 & 0.42575 & 0.41154 & 0.42833 & 0.42627 & 0.42575 & 0.42575 & 0.42575 & 0.42575 \\
0.1 & 0.55252 & 0.47433 & 0.51790 & 0.53613 & 0.55258 & 0.55258 & 0.55274 & 0.55252 \\
0.2 & 0.61247 & 0.52131 & 0.58137 & 0.60498 & 0.61283 & 0.61283 & 0.61248 & 0.61281 \\
0.3 & 0.64797 & 0.55389 & 0.62357 & 0.64673 & 0.64803 & 0.64803 & 0.64798 & 0.64801 \\
0.4 & 0.66722 & 0.57306 & 0.64768 & 0.66917 & 0.66720 & 0.66720 & 0.66724 & 0.66719 \\
0.5 & 0.67336 & 0.57938 & 0.65553 & 0.67625 & 0.67332 & 0.67332 & 0.67337 & 0.67331 \\
\hline$E_{\max }(\%)$ & & 14.883 & 6.265 & 2.966 & 0.060 & 0.060 & 0.041 & 0.056 \\
\hline$E_{r}(\%)$ & & 12.493 & 3.548 & 0.871 & 0.015 & 0.015 & 0.009 & 0.013 \\
\hline
\end{tabular}

\subsubsection{Two-region source problem with reflective boundary conditions}

This problem consists of a one dimensional slab with two regions and one-energy group. Both regions measure $1 \mathrm{~cm}$ in length but only region one has a fixed source, as fig.3.52 shows. The problem does not consider scattering and fission. Total cross-section is $\Sigma_{t}=1 \mathrm{~cm}^{-1}$ for both regions. The boundary conditions are reflective at both outer boundaries. Different spatial nodalization has been considered: 10, 100 and 200 nodes. Tables 3.15 and 3.16 show the scalar fluxes com- 
pared with the analytical transport solution. Similarly to the previous problem, when the spatial discretization or discrete ordinates order increases the solution is closer to the analytical value.

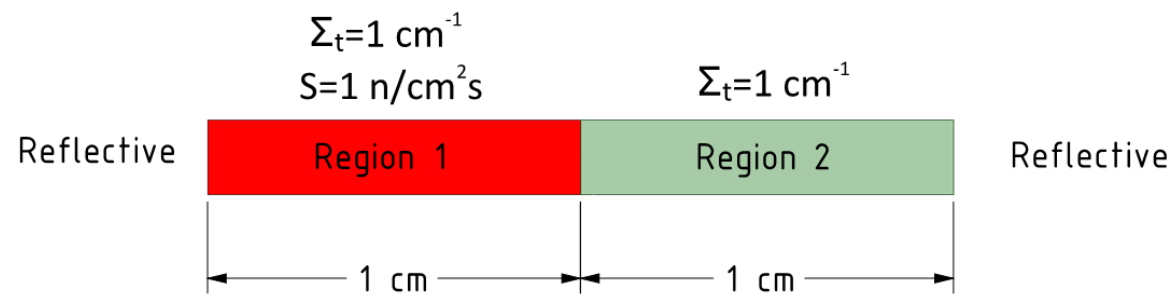

Figure 3.52: Two-region source problem with reflective boundary conditions.

Table 3.15: Scalar fluxes for the two-region source problem with reflective boundary conditions and 10 spatial cells for different $S_{N}$.

\begin{tabular}{lccccccc}
\hline$x(\mathrm{~cm})$ & Ref. & $S_{2}$ & $S_{4}$ & $S_{8}$ & $S_{16}$ & $S_{32}$ & $S_{64}$ \\
\hline 0 & 0.86124 & 0.82914 & 0.86731 & 0.86284 & 0.86171 & 0.86180 & 0.86180 \\
0.4 & 0.83096 & 0.78625 & 0.82990 & 0.83352 & 0.83174 & 0.83168 & 0.83165 \\
0.8 & 0.70368 & 0.63603 & 0.67350 & 0.69687 & 0.70669 & 0.70603 & 0.70279 \\
1.2 & 0.29631 & 0.36396 & 0.32649 & 0.30312 & 0.29330 & 0.29396 & 0.29720 \\
1.6 & 0.16904 & 0.21374 & 0.17009 & 0.16647 & 0.16825 & 0.16831 & 0.16834 \\
2 & 0.13876 & 0.17085 & 0.13269 & 0.13715 & 0.13828 & 0.13819 & 0.13819 \\
\hline$E_{\max }(\%)$ & & 26.447 & 10.186 & 2.301 & 1.014 & 0.793 & 0.409 \\
\hline$E_{r}(\%)$ & & 15.188 & 3.384 & 1.073 & 0.400 & 0.353 & 0.232 \\
\hline
\end{tabular}

Table 3.16: Scalar fluxes for the two-region source problem with reflective boundary conditions and 100 spatial cells for different $S_{N}$ (* corresponds to calculation with 200 spatial cells).

\begin{tabular}{lcccccccc}
\hline x $(\mathrm{cm})$ & Ref. & $S_{2}$ & $S_{4}$ & $S_{8}$ & $S_{16}$ & $S_{32}$ & $S_{64}$ & $* 200 / S_{32}$ \\
\hline 0 & 0.86124 & 0.82845 & 0.86648 & 0.86228 & 0.86117 & 0.86125 & 0.86124 & 0.86124 \\
0.4 & 0.83096 & 0.78560 & 0.82895 & 0.83262 & 0.83105 & 0.83096 & 0.83097 & 0.83095 \\
0.8 & 0.70368 & 0.63566 & 0.67265 & 0.69499 & 0.70376 & 0.70404 & 0.70368 & 0.70402 \\
1.2 & 0.29631 & 0.36433 & 0.32734 & 0.30500 & 0.29623 & 0.29595 & 0.29631 & 0.29597 \\
1.6 & 0.16904 & 0.21439 & 0.17104 & 0.16737 & 0.16894 & 0.16904 & 0.16903 & 0.16904 \\
2 & 0.13876 & 0.17154 & 0.13351 & 0.13771 & 0.13882 & 0.13874 & 0.13875 & 0.13875 \\
\hline$E_{\max }(\%)$ & & 26.829 & 10.473 & 2.933 & 0.056 & 0.118 & 0.006 & 0.056 \\
\hline$E_{r}(\%)$ & & 15.390 & 3.449 & 1.038 & 0.027 & 0.03 & 0.003 & 0.013 \\
\hline
\end{tabular}




\subsubsection{Two-group 1D anisotropic scattering problem}

This case was selected because it considers a slab with two regions, two energy groups, and anisotropic scattering [Ju et al., 2007]. As shown in fig.3.53, the problem geometry is composed of one region of $l_{1}=2 \mathrm{~cm}$ with fixed source and other of $l_{2}=18 \mathrm{~cm}$ without source. The cross sections of the problem are found in [Capilla et al., 2016]. On the left boundary the condition is reflective and on the right is vacuum. Table 3.17 gives the scalar fluxes for the first energy group using discrete ordinates order 2, 4, 8 and 16. The executed cases with the reference PARTISN and n-DOTEC use the same discretization (320 meshes) and discrete ordinates order $S_{16}$. Table 3.17 also shows the maximum relative error and the mean relative error compared with the reference values. Table 3.18 shows the same for the second energy group. The results calculated with n-DOTEC are practically the same value as those calculated with PARTISN.

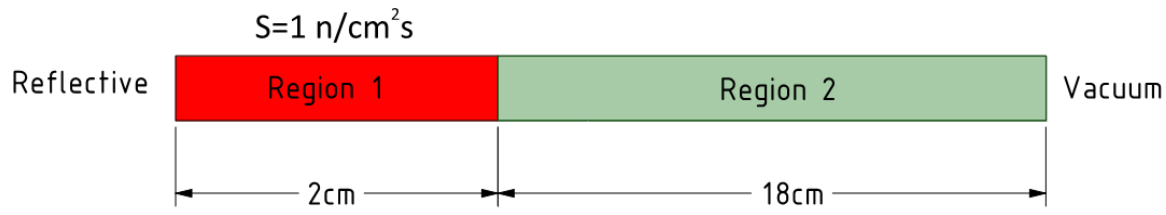

Figure 3.53: Two-group anisotropic scattering problem.

Table 3.17: Scalar fluxes for the first group of the anisotropic problem using different $S_{N}$.

\begin{tabular}{lccccc}
\hline $\mathrm{x}(\mathrm{cm})$ & Ref. Partisn $S_{16}$ & $S_{2}$ & $S_{4}$ & $S_{8}$ & $S_{16}$ \\
\hline 0.125 & 1.75955 & 1.70306 & 1.76547 & 1.75935 & 1.75955 \\
0.375 & 1.74714 & 1.68596 & 1.75285 & 1.74703 & 1.74714 \\
1.875 & 1.17696 & 1.08756 & 1.13290 & 1.16183 & 1.17695 \\
2.125 & 0.77811 & 0.86871 & 0.82202 & 0.79325 & 0.77811 \\
2.375 & 0.55566 & 0.68374 & 0.59071 & 0.55886 & 0.55566 \\
2.625 & 0.42064 & 0.53816 & 0.43576 & 0.41889 & 0.42064 \\
18.875 & $7.7930 \mathrm{E}-07$ & $9.3222 \mathrm{E}-08$ & $7.2296 \mathrm{E}-07$ & $7.7934 \mathrm{E}-07$ & $7.7930 \mathrm{E}-07$ \\
19.125 & $6.3709 \mathrm{E}-07$ & $7.3111 \mathrm{E}-08$ & $5.8912 \mathrm{E}-07$ & $6.3714 \mathrm{E}-07$ & $6.3709 \mathrm{E}-07$ \\
19.375 & $5.1925 \mathrm{E}-07$ & $5.7209 \mathrm{E}-08$ & $4.7825 \mathrm{E}-07$ & $5.1956 \mathrm{E}-07$ & $5.1925 \mathrm{E}-07$ \\
19.625 & $4.2073 \mathrm{E}-07$ & $4.4603 \mathrm{E}-08$ & $3.8550 \mathrm{E}-07$ & $4.2035 \mathrm{E}-07$ & $4.2073 \mathrm{E}-07$ \\
19.875 & $3.3532 \mathrm{E}-07$ & $3.4566 \mathrm{E}-08$ & $3.0640 \mathrm{E}-07$ & $3.3436 \mathrm{E}-07$ & $3.3532 \mathrm{E}-07$ \\
\hline$E_{\max }(\%)$ & & 89.691 & 8.625 & 1.944 & 0.0000578 \\
\hline$E_{r}(\%)$ & & 47.415 & 5.418 & 0.420 & 0.0000187 \\
\hline
\end{tabular}


Table 3.18: Scalar fluxes for the second group of the anisotropic problem using different $S_{N}$.

\begin{tabular}{lccccc}
\hline $\mathrm{x}(\mathrm{cm})$ & Ref. Partisn $S_{16}$ & $S_{2}$ & $S_{4}$ & $S_{8}$ & $S_{16}$ \\
\hline 0.125 & 3.20472 & 2.94440 & 3.21688 & 3.20449 & 3.20472 \\
0.375 & 3.17772 & 2.91174 & 3.18831 & 3.17770 & 3.17772 \\
1.875 & 2.17197 & 2.01508 & 2.11367 & 2.15317 & 2.17197 \\
2.125 & 1.63787 & 1.76087 & 1.69666 & 1.65668 & 1.63786 \\
2.375 & 1.28993 & 1.51997 & 1.34944 & 1.29594 & 1.28993 \\
2.625 & 1.04771 & 1.30182 & 1.08215 & 1.04610 & 1.04771 \\
18.875 & $7.2189 \mathrm{E}-06$ & $1.4113 \mathrm{E}-06$ & $6.8972 \mathrm{E}-06$ & $7.2191 \mathrm{E}-06$ & $7.2189 \mathrm{E}-06$ \\
19.125 & $5.9559 \mathrm{E}-06$ & $1.1205 \mathrm{E}-06$ & $5.6749 \mathrm{E}-06$ & $5.9564 \mathrm{E}-06$ & $5.9559 \mathrm{E}-06$ \\
19.375 & $4.8957 \mathrm{E}-06$ & $8.8726 \mathrm{E}-07$ & $4.6479 \mathrm{E}-06$ & $4.8955 \mathrm{E}-06$ & $4.8957 \mathrm{E}-06$ \\
19.625 & $3.9960 \mathrm{E}-06$ & $6.9972 \mathrm{E}-07$ & $3.7750 \mathrm{E}-06$ & $3.9915 \mathrm{E}-06$ & $3.9960 \mathrm{E}-06$ \\
19.875 & $3.1994 \mathrm{E}-06$ & $5.4827 \mathrm{E}-07$ & $3.0169 \mathrm{E}-06$ & $3.1883 \mathrm{E}-06$ & $3.1994 \mathrm{E}-06$ \\
\hline$E_{\max }(\%)$ & & 82.863 & 5.704 & 1.148 & 0.0000245 \\
\hline$E_{r}(\%)$ & & 44.190 & 3.669 & 0.283 & 0.0000057 \\
\hline
\end{tabular}

\subsubsection{Two-dimensional one-energy group problem}

This two dimensional problem is made up of three regions shown in Fig.3.54. The system geometry consist of a $[0,100] \times[0,100] \mathrm{cm}^{2}$ square domain with a isotropic fixed source located at $[0,10] \times[0,10] \mathrm{cm}^{2}$ with a value of $S=1 \mathrm{n} / \mathrm{cm}^{2} s$. The region between the source and region 3 corresponds to a void region located at $\left[[10,50] \times[10,50] \mathrm{cm}^{2}\right.$. There is no scattering and fission. Total cross-sections for each region and the boundary conditions (reflective and vacuum) are shown in Fig.3.54. This problem has been inspired in the Kobayashi's problem 1 collapsed to a two-dimensional case [Kobayashi, 1997]. Two different meshes are considered, one of $26 \times 26$ and other of $52 \times 52$. The reference values were calculated by TWODANT code with a mesh of $600 \times 600$ mesh intervals, $S_{16}$ discrete ordinate order and a convergence criteria of $10^{-7}$ [Capilla et al., 2016]. A comparison of the results with the reference is given in the Table 3.19 showing values for different spatial discretization and $S_{N}$ orders. In this case the errors calculated are the maximum absolute error and the mean error. As shown in previous problems, the higher the number of cells and the discrete ordinates order, the more accurate the results. However, some discrepancies are appreciated when the value of scalar flux is close to zero. 


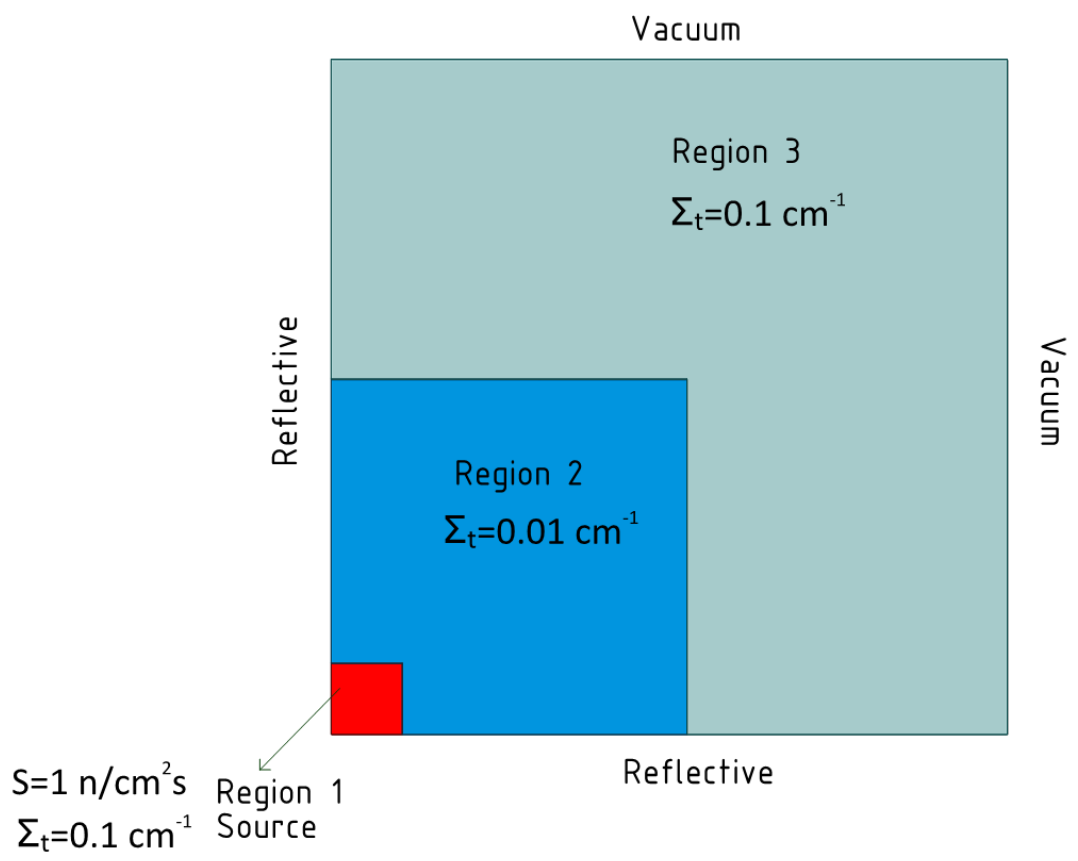

Figure 3.54: Two-dimensional problem.

Table 3.19: Scalar fluxes along $y=5$ for the two-dimensional problem with $26 \times 26$ and $52 \times 52$ cells discretization for different $S_{N}$ orders.

\begin{tabular}{lccccc}
\hline $\mathrm{x}(\mathrm{cm})$ & Ref. $600 \times 600 S_{16}$ & $26 \times 26 S_{16}$ & $26 \times 26 S_{32}$ & $52 \times 52 S_{16}$ & $52 \times 52 S_{20}$ \\
\hline 5 & 6.77540 & 6.83419 & 6.84229 & 6.79931 & 6.78679 \\
15 & 2.09770 & 1.94675 & 1.89264 & 2.05572 & 2.06019 \\
25 & 1.02020 & 1.08189 & 1.01391 & 1.03775 & 1.03828 \\
35 & 0.62759 & 0.64211 & 0.65924 & 0.61754 & 0.62513 \\
45 & 0.39092 & 0.41648 & 0.47001 & 0.38459 & 0.41261 \\
55 & 0.13633 & 0.15201 & 0.19983 & 0.14372 & 0.16046 \\
65 & 0.03280 & 0.04882 & 0.06941 & 0.03162 & 0.03380 \\
75 & 0.01017 & 0.02905 & 0.03830 & 0.00262 & 0.00506 \\
85 & 0.00317 & 0.02216 & 0.02658 & 0.00563 & 0.00259 \\
95 & 0.00103 & 0.01948 & 0.02179 & 0.00551 & 0.00019 \\
\hline Max. error & & 0.151 & 0.205 & 0.042 & 0.038 \\
\hline Mean error & & 0.040 & 0.056 & 0.012 & 0.012 \\
\hline
\end{tabular}




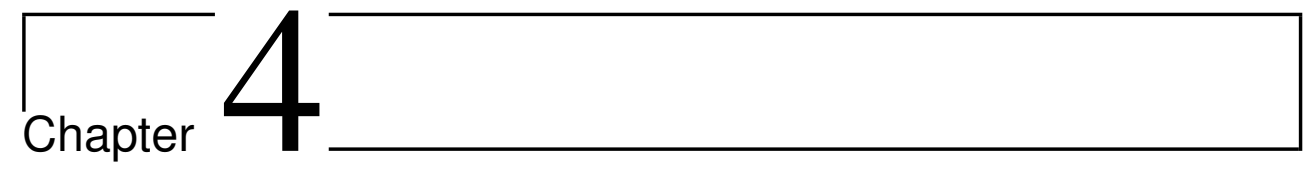

\section{Neutron Diffusion Equation}

This chapter is focused on the Neutron Diffusion approximation to the neutron transport equation for $1 D, 2 D$ and $3 D$ systems modeled with Cartesian geometry by means of the finite difference method to discretize the spatial variables. The method solves the steady-state diffusion equation using the Krylov-schur method to solve generalized eigenvalue problems. A simple formulation of the neutron diffusion equation using two finite difference schemes is defined. The numerical results of the neutron diffusion equation are compared with the previous results obtained by the discrete ordinates method.

\subsection{Introduction to the Neutron Diffusion Equation}

The neutron diffusion equation is the simplest approximation to the neutron transport equation which has been commonly used in research and reactor core simulations. The main property that the diffusion theory establish is that the neutron current is proportional to the gradient of the scalar flux, and this coefficient of proportionality is the diffusion coefficient. This asumption is also known with the name of Fick's law:

$$
J(x)=-D(x) \frac{\partial \phi(x)}{\partial x}
$$


The next section will derive the diffusion equation starting from neutron transport equation presented in last chapter. Furthermore, this derivation explains how the definition of the Fick's law and the diffusion coefficient are defined.

The most important advantage of the diffusion equation is the elimination of the angular variable $\hat{\Omega}$, thereby reducing the computational resources to solve the approximate problem. The diffusion solution is accurate only in cases where the angular flux is weak space dependent. In many problems, it is necessary an homogenization process which consist in approximating the heterogeneous real problem by a fictitious homogeneous problem. To do that, it is required to define homogenized cross sections and homogenized diffusion coefficients, and the optimal definition of these parameters is very discussed due to, even though different definitions work well in some particular cases [Larsen and Hughes, 1980], there is no one definition valid for any application. In any case, this discussion is beyond the scope of this thesis.

Therefore, although the diffusion equation allows full-core calculations with reasonable computational time and accuracy. The diffusion approximation is valid only under 4 assumptions Stacey [2007]. First, the neutron current is proportional to the neutron flux gradient. Second, there is much less neutron absorption than scattering. Third, it is considered that the variation of the space neutron distribution is linear. Fourth, the scattering is assumed isotropic.

\subsection{Derivation of the Neutron Diffusion Equation}

The derivation of the diffusion equation can be obtained by using a simplified version of asymtotic derivations for a 1D planar geometry problem. This derivation was publish by several authors ([Larsen, 1980], [Larsen et al., 1996], [Habetler and Matkowsky, 1975], [Papanicolaou, 1975]). The 1D case allows the derivation by using Legendre polynomials and the derivation can be extrapolated to the $2 \mathrm{D}$ and $3 \mathrm{D}$.

The starting point is the one dimensional neutron transport equation with anisotropic scattering: 


$$
\begin{array}{r}
\mu \frac{\partial \psi}{\partial x}(x, \mu, E)+\Sigma_{t}(x, E) \psi(x, \mu, E) \\
=\sum_{l=0}^{\infty} \frac{2 l+1}{2} P_{l}(\mu) \int_{0}^{\infty} \int_{-1}^{1} P_{l}\left(\mu^{\prime}\right) \Sigma_{s, l}\left(x, E^{\prime} \rightarrow E\right) \psi\left(x, \mu^{\prime}, E^{\prime}\right) d \mu^{\prime} d E^{\prime} \\
+\frac{Q(x, E)}{2}
\end{array}
$$

First it is necessary to consider an infinite homogeneous medium, having no spatial dependence:

$$
\begin{array}{r}
\Sigma_{t}(E) \psi(\mu, E) \\
=\sum_{l=0}^{\infty} \frac{2 l+1}{2} P_{l}(\mu) \int_{0}^{\infty} \int_{-1}^{1} P_{l}\left(\mu^{\prime}\right) \Sigma_{s, l}\left(E^{\prime} \rightarrow E\right) \psi\left(\mu^{\prime}, E^{\prime}\right) d \mu^{\prime} d E^{\prime} \\
+\frac{Q(E)}{2}
\end{array}
$$

The exact solution is defined by operating by $\int_{-1}^{1} P_{n}(\mu)(\cdot) d \mu$ and considering the $n$th Legendre moment of the angular flux and the Legendre polynomials properties:

$$
\phi_{n}(E)=\int_{-1}^{1} P_{n}(\mu) \psi(\mu, E) d \mu, \quad \int_{-1}^{1} P_{n}(\mu) P_{l}(\mu) d \mu=\delta_{l, n} .
$$

So, the system of equations is:

$$
\Sigma_{t}(E) \phi_{n}(E)=\int_{0}^{\infty} \Sigma_{s, n}\left(E^{\prime} \rightarrow E\right) \phi_{n}\left(E^{\prime}\right) d E^{\prime}+Q(E) \delta_{n, 0}, \quad 0 \leq n .
$$

Therefore, if $n \geq 1, \phi_{n}(E)=0$, and:

$$
\psi(\mu, E)=\sum_{n=0}^{\infty} \frac{2 n+1}{2} P_{n}(\mu) \phi_{n}(E)=\frac{1}{2} \phi_{0}(E),
$$


where $\phi_{0}(E)$ satisfies 4.4 with $n=0$. It can be appreciated in Equations 4.34.5 that for an infinite, spatially uniform problem with an isotropic source, the angular flux is independent of both space $(\mathrm{x})$ and angle $(\mu)$.

Physically, the net leakage rate from a spatial increment $d x$ can be assumed to be small compared with the collision rate or scattering rates in $d x$. So, returning to the eq.4.2 and introducing the symbol $\varepsilon$ to describe that the spatial derivative in $\psi$ si small in comparison to the other terms:

$$
\begin{array}{r}
\varepsilon \mu \frac{\partial \psi}{\partial x}(x, \mu, E)+\Sigma_{t}(E) \psi(\mu, E) \\
=\sum_{l=0}^{\infty} \frac{2 l+1}{2} P_{l}(\mu) \int_{0}^{\infty} \int_{-1}^{1} P_{l}\left(\mu^{\prime}\right) \Sigma_{s, l}\left(E^{\prime} \rightarrow E\right) \psi\left(\mu^{\prime}, E^{\prime}\right) d \mu^{\prime} d E^{\prime} \\
+\frac{Q(E)}{2}
\end{array}
$$

Due to the fact that $\psi$ has a weak spatial dependence, it is normal to expect that the angular dependence is also weak. In this way,as the spatial derivative of $p s i$ limits to zero, $\psi$ should become isotropic. Following this reasoning the solution of the eq.4. 6 has the Legendre polynomial expansion:

$$
\psi(x, \mu, E)=\sum_{l=0}^{\infty} \frac{2 l+1}{2} \varepsilon^{l} P_{l}(\mu) \phi_{l}(E),
$$

where $\phi_{l}(E)=O(1)$ for all $l$. Introducing eq.4.7 into eq.4.6, using the following identity and operating by $\int_{-1}^{1} P_{n}(\mu)(\cdot) d \mu$ :

$$
\mu P_{l}(\mu)=\frac{l+1}{2 l+1} P_{l+1}(\mu)+\frac{l}{2 l+1} P_{l-1}(\mu),
$$

one can define for all $n \geq 0$ :

$$
\begin{aligned}
\frac{n}{2 n+1} \frac{\partial \phi_{n-1}}{\partial x}(x, E) & +\varepsilon^{2} \frac{n+1}{2 n+1} \frac{\partial \phi_{n+1}}{\partial x}(x, E)+\Sigma_{t}(x, E) \phi_{n}(x, E) \\
= & \int_{0}^{\infty} \Sigma_{s, n}\left(x, E^{\prime} \rightarrow E\right) \phi_{n}\left(x, E^{\prime}\right) d E^{\prime}+\delta_{n, 0} Q(x, E)
\end{aligned}
$$


For $n=0$ and $n=1$ the following two equations are obtained:

$$
\begin{aligned}
& \varepsilon^{2} \frac{\partial \phi_{1}}{\partial x}(x, E)+\Sigma_{t}(x, E) \phi_{0}(x, E)=\int_{0}^{\infty} \Sigma_{s, 0}\left(x, E^{\prime} \rightarrow E\right) \phi_{0}\left(x, E^{\prime}\right) d E^{\prime}+Q(x, E) \\
& \frac{1}{3} \frac{\partial \phi_{0}}{\partial x}(x, E)+\frac{2 \varepsilon^{2}}{3} \frac{\partial \phi_{2}}{\partial x}+\Sigma_{t}(x, E) \phi_{1}(x, E)=\int_{0}^{\infty} \Sigma_{s, 1}\left(x, E^{\prime} \rightarrow E\right) \phi_{1}\left(x, E^{\prime}\right) d E^{\prime}
\end{aligned}
$$

In order to derive the diffusion equation, it is necessary to consider the following two assumptions:

$$
\varepsilon^{2} \frac{\partial \phi_{2}}{\partial x}(x, E) \approx 0, \quad \int_{0}^{\infty} \Sigma_{s, 1}\left(x, E^{\prime} \rightarrow E\right) d E^{\prime}=\Sigma_{s, 1}\left(x, E^{\prime}\right)
$$

Introducing both approximations eq.4.11 is expressed as:

$$
\frac{1}{3} \frac{\partial \phi_{0}}{\partial x}(x, E)+\Sigma_{t}(x, E) \phi_{1}(x, E)=\Sigma_{s, 1}(x, E) \phi_{1}(x, E)
$$

Defining the transport cross section:

$$
\Sigma_{t r}(x, E)=\Sigma_{t}(x, E)-\Sigma_{s, 1}(x, E)
$$

then eq.4.13 produces the Fick's law:

$$
\phi_{1}(x, E)=-\frac{1}{3 \Sigma_{t r}(x E)} \frac{\partial \phi_{0}}{\partial x}(x, E)
$$

Introducing the eq.4.15 into eq.4.10 and setting $\varepsilon=1$ the diffusion equation is defined as:

$$
\begin{aligned}
-\frac{\partial}{\partial x} \frac{1}{3 \Sigma_{t r}(x, E)} \frac{\partial \phi_{0}}{\partial x} & (x, E)+\Sigma_{t}(x, E) \phi_{0}(x, E) \\
& =\int_{0}^{\infty} \Sigma_{s, 0}\left(x, E^{\prime} \rightarrow E\right) \phi_{0}\left(x, E^{\prime}\right) d E^{\prime}+Q(x, E) .
\end{aligned}
$$


Finally, considering a multigroup notation and taking $\Sigma_{t r}=\Sigma_{t}$, the diffusion equation in multidimensional form can be written as [Cacuci, 2010]:

$$
-\nabla\left(D_{g} \nabla \phi_{g}\right)+\Sigma_{r, g} \phi_{g}=\sum_{g^{\prime} \neq g}^{G} \Sigma_{s, g^{\prime} \rightarrow g} \phi_{g^{\prime}}+\frac{\chi_{g}}{k_{e f f}} \sum_{g^{\prime}=1}^{G} \Sigma_{f, g} \phi_{g^{\prime}}
$$

Where

$$
D_{g}: \text { diffusion coefficient of group } g
$$

$$
D_{g}=\frac{1}{3 \Sigma_{t, g}}
$$

$\Sigma_{r, g}$ : removal cross section of group $g$ defined by

the sumation of absorption and out-scatter cross section.

$$
\Sigma_{r, g}=\Sigma_{a, g}+\sum_{g^{\prime} \neq g} \Sigma_{s, g \rightarrow g^{\prime}}=\Sigma_{t, g}-\Sigma_{s, g \rightarrow g}
$$

$\chi_{g}$ : fission spectrum of group $g$

$k_{\text {eff }}:$ multiplication factor

$\nu \Sigma_{f, g}$ : production cross section of group $g$

$\Sigma_{s, g^{\prime} \rightarrow g}$ : scattering cross section from group $g^{\prime}$ to $g$

$\Sigma_{t, g}:$ total cross section of group $g$

$\phi_{g}:$ neutron flux in group $g$

\subsection{D Finite Difference Diffusion Equation using Cell-Centered Scheme}

Another easier and more understandable way to derive the 1D neutron diffusion equation can be defined expanding the angular flux $\psi(x, \mu)$ in terms of the director cosine $\mu$ by $P_{1}$-approximation, the energy variable is not considered for notation simplification:

$$
\psi(x, \mu)=\sum_{l=0}^{\infty}(2 l+1) \phi_{l}(x) P_{l}(\mu) \approx \phi_{0}(x)+3 \mu \phi_{1}(x)
$$


$P_{1}$ represents the order of the expansion using Legendre Polynomials $P_{l}$ as the expansion function. Considering the orthogonality relation of the Legendre Polynomials:

$$
\int_{-1}^{1} P_{k}(\mu) P_{l}(\mu) d \mu=\frac{1}{2 l+1} \delta_{k l}
$$

with $\delta_{k l}$ Kronecker delta, equal to 1 if $k=l$ and 0 otherwise, the expansion coefficients are given by:

$$
\phi_{l}(x)=\frac{1}{2} \int_{-1}^{1} P_{l}(\mu) \psi(x, \mu) d \mu
$$

And knowing that $P_{0}(\mu)=1$ and $P_{1}(\mu)=\mu$, it easy to obtain:

$$
\phi_{0}(x)=\frac{1}{2} \int_{-1}^{1} \psi(x, \mu) d \mu \equiv \phi(x) ; \quad \phi_{1}(x)=\frac{1}{2} \int_{-1}^{1} \mu \psi(x, \mu) d \mu \equiv J(x)
$$

As can be seen, the coefficients of the $P_{1}$-expansion are the scalar flux $\phi(x)$ and the current $J(x)$ by definition in the diffusion theory.

Substituting the coefficients of the eq.4.21 into the eq.4.18, the angular flux is defined as:

$$
\psi(x, \mu)=\phi(x)+3 \mu J(x)
$$

If this expression is introduced into the isotropic neutron transport equation for a given energy group eq.4.23:

$$
\mu \frac{\partial \psi(x, \mu)}{\partial x}+\Sigma_{t}(x) \psi(x, \mu)=\Sigma_{s}(x) \phi(x)+Q(x)
$$

one can obtain eq.4.24 


$$
\begin{aligned}
\frac{1}{2} \int_{-1}^{1} \mu \frac{\partial}{\partial x}[\phi(x)+3 \mu J(x)] d \mu+ & \frac{1}{2} \int_{-1}^{1} \Sigma_{t}(x)[\phi(x)+3 \mu J(x)] d \mu= \\
& \frac{1}{2} \int_{-1}^{1} \Sigma_{s}(x) \phi(x) d \mu+\frac{1}{2} \int_{-1}^{1} Q(x) d \mu
\end{aligned}
$$

Integrating:

$$
\begin{gathered}
\frac{\partial}{\partial x} \frac{1}{2} \int_{-1}^{1} \mu[\phi(x)+3 \mu J(x)] d \mu=\frac{\partial}{\partial x} \frac{1}{2}\left(\phi(x) \int_{-1}^{1} \mu d \mu+J(x) \int_{-1}^{1} 3 \mu^{2} d \mu\right)= \\
\frac{\partial}{\partial x} \frac{1}{2}\left(\left.\phi(x) \frac{\mu^{2}}{2}\right|_{-1} ^{1}+\left.J(x) \frac{3 \mu^{3}}{3}\right|_{-1} ^{1}\right)=\frac{\partial}{\partial x} \frac{1}{2}\left(\phi(x)\left[\frac{1}{2}-\frac{1}{2}\right]+J(x)[1-(-1)]\right)= \\
\frac{1}{2} \int_{-1}^{1} \Sigma_{t}(x)[\phi(x)+3 \mu J(x)] d \mu=\frac{1}{2} \Sigma_{t}(x)\left(\phi(x) \int_{-1}^{1} d \mu+J(x)\right)=\frac{\partial}{\partial x} J(x) \quad \text { (4.25) } \\
\frac{1}{2} \Sigma_{t}(x)\left(\left.\phi(x) \mu\right|_{-1} ^{1}+\left.J(x) \frac{3 \mu^{2}}{2}\right|_{-1} ^{1}\right)=\Sigma_{t}(x) \phi(x) \\
\frac{1}{2} \int_{-1}^{1} \Sigma_{s}(x) \phi(x) d \mu=\frac{1}{2} \Sigma_{s}(x) \phi(x) \int_{-1}^{1} d \mu=\Sigma_{s} \phi(x) \\
\frac{1}{2} \int_{-1}^{1} Q(x) d \mu=Q(x)
\end{gathered}
$$

Then, eq.4.24 can be written as:

$$
\frac{\partial}{\partial x} J(x)+\Sigma_{t} \phi(x)=\Sigma_{s} \phi(x)+Q(x)
$$

Considering $\Sigma_{a}=\Sigma_{t}-\Sigma_{s}$ :

$$
\frac{\partial}{\partial x} J(x)+\Sigma_{a} \phi(x)=Q(x)
$$


If eq.4.23 is multiplied by $\mu$ and integrated over $-1 \leq \mu \leq 1$ the following equation is derived:

$$
\frac{1}{3} \frac{\partial \phi(x)}{\partial x}+\Sigma_{t} J(x)=0
$$

If the current is isolated to one side of the equation and everything else to the other side, one can define the Fick's law:

$$
J(x)=-\frac{1}{3 \Sigma_{t}} \frac{\partial \phi(x)}{\partial x}=-D \frac{\partial \phi(x)}{\partial x}
$$

where $D$ is the diffusion coefficient. Substituting eq.4.32 into eq.4.30 it can be written the well known neutron diffusion equation:

$$
-\frac{\partial}{\partial x}\left[D \frac{\partial}{\partial x}\right]+\Sigma_{a}(x) \phi(x)=Q(x)
$$

Introducing multigroup notation and considering the removal cross section $\Sigma_{r, g}=$ $\Sigma_{a, g}+\sum_{g^{\prime} \neq g} \Sigma_{s, g \rightarrow g^{\prime}}=\Sigma_{t, g}-\Sigma_{s, g \rightarrow g}$, the one-dimensional steady-state neutron diffusion equations is:

$$
\begin{gathered}
-\frac{\partial}{\partial x}\left(D_{g}(x) \frac{\partial}{\partial x} \phi_{g}(x)\right)+\Sigma_{r, g}(x) \phi_{g}(x)= \\
\sum_{g^{\prime} \neq g}^{G} \Sigma_{s, g^{\prime} \rightarrow g}(x) \phi_{g^{\prime}}(x)+\frac{\chi_{g}(x)}{k_{\text {eff }}} \sum_{g^{\prime}=1}^{G} \Sigma_{f, g}(x) \phi_{g^{\prime}}(x)
\end{gathered}
$$

Considering the Fick's Law: $J(x)_{g}=-D_{g}(x) \frac{\partial \phi_{g}(x)}{\partial x}$

$$
\frac{\partial}{\partial x} J(x)_{g}+\Sigma_{r, g}(x) \phi_{g}(x)=\sum_{g^{\prime} \neq g}^{G} \Sigma_{s, g^{\prime} \rightarrow g}(x) \phi_{g^{\prime}}(x)+\frac{\chi_{g}(x)}{k_{e f f}} \sum_{g^{\prime}=1}^{G} \Sigma_{f, g}(x) \phi_{g^{\prime}}(x)
$$

Then, if one considers the integral over the interval $\left[x_{i-1}, x_{i}\right]$ and $J_{i}$ the current in the point $x_{i}$ as shown in fig.4.1, it can be written: 


$$
\begin{aligned}
& J_{i, g}-J_{i-1, g}+\int_{x_{i-1}}^{x_{i}} \Sigma_{r, g}(x) \phi_{g}(x) d x= \\
& \quad \int_{x_{i-1}}^{x_{i}} \sum_{g^{\prime} \neq g}^{G} \Sigma_{s, g^{\prime} \rightarrow g}(x) \phi_{g^{\prime}}(x) d x+\int_{x_{i-1}}^{x_{i}} \frac{\chi_{g}(x)}{k_{e f f}} \sum_{g^{\prime}=1}^{G} \nu \Sigma_{f, g}(x) \phi_{g^{\prime}}(x) d x \\
& J_{i, g}-J_{i-1, g}+\Sigma_{r, i, g} h_{i} \phi_{i, g}=\sum_{g^{\prime} \neq g}^{G} \Sigma_{s, i, g^{\prime} \rightarrow g} h_{i} \phi_{i, g^{\prime}}+\frac{\chi_{i, g}}{k_{e f f}} \sum_{g^{\prime}=1}^{G} \nu \Sigma_{f, i, g} h_{i} \phi_{i, g^{\prime}}
\end{aligned}
$$

Rearranging:

$$
\frac{J_{i, g}}{h_{i}}-\frac{J_{i-1, g}}{h_{i}}+\Sigma_{r, i, g} \phi_{i, g}=\sum_{g^{\prime} \neq g}^{G} \Sigma_{s, i, g^{\prime} \rightarrow g} \phi_{i, g^{\prime}}+\frac{\chi_{i, g}}{k_{e f f}} \sum_{g^{\prime}=1}^{G} \nu \Sigma_{f, i, g} \phi_{i, g^{\prime}}
$$

Fig.4.1 shows the cell-centered finite difference scheme. The current terms from this scheme can be defined as:

$$
\begin{gathered}
J_{i, g}^{R}=-D_{i, g} \frac{\phi_{i, g}^{R}-\phi_{i, g}}{h_{i} / 2}, \\
J_{i+1, g}^{L}=-D_{i+1, g} \frac{\phi_{i+1, g}-\phi_{i+1, g}^{L}}{h_{i+1} / 2},
\end{gathered}
$$

with the interface conditions:

$$
\begin{aligned}
& J_{i}^{R}=J_{i+1}^{L}=J_{i}, \\
& \phi_{i}^{R}=\phi_{i+1}^{L}=\phi_{s} .
\end{aligned}
$$

Then, from eq.4.41 it can be obtained the relation:

$$
-D_{i, g} \frac{\phi_{s, g}-\phi_{i, g}}{h_{i} / 2}=-D_{i+1, g} \frac{\phi_{i+1, g}-\phi_{s, g}}{h_{i+1} / 2},
$$

If one isolates $\phi_{s, g}$ obtains: 


$$
\begin{aligned}
& \phi_{s, g}=\frac{D_{i, g} / h_{i}}{D_{i, g} / h_{i}+D_{i+1, g} / h_{i+1}} \phi_{i, g}+\frac{D_{i+1, g} / h_{i+1}}{D_{i, g} / h_{i}+D_{i+1, g} / h_{i+1}} \phi_{i+1, g}= \\
& \omega_{i, g} \phi_{i, g}+\left(1-\omega_{i, g}\right) \phi_{i+1, g} \text {. }
\end{aligned}
$$

Substituting the $\phi_{s}$ into 4.39 and 4.40 one can define the diffusion coupled coefficients as:

$$
\begin{aligned}
& \widetilde{D}_{i, g}^{x+}=\frac{2 D_{i, g} D_{i+1, g}}{\left(D_{i, g} h_{i+1}+D_{i+1, g} h_{i}\right) h_{i}} \\
& \widetilde{D}_{i, g}^{x-}=\frac{2 D_{i-1, g} D_{i, g}}{\left(D_{i-1, g} h_{i}+D_{i, g} h_{i-1}\right) h_{i}}
\end{aligned}
$$

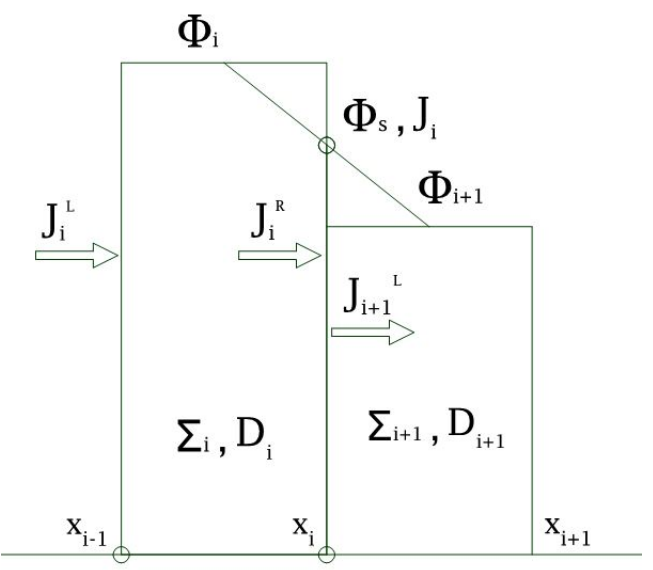

Figure 4.1: Cell-centered finite difference scheme.

So, the currents of fig.4.1 can also be defined as:

$$
\begin{gathered}
\frac{J_{i, g}}{h_{i}}=\frac{J_{i, g}^{R}}{h_{i}}=-\widetilde{D}_{i, g}^{x+}\left[\phi_{i+1, g}-\phi_{i, g}\right] \\
\frac{J_{i-1, g}}{h_{i}}=\frac{J_{i, g}^{L}}{h_{i}}=-\widetilde{D}_{i, g}^{x-}\left[\phi_{i, g}-\phi_{i-1, g}\right]
\end{gathered}
$$


Finally the discretized neutron diffusion equation is written in eq.4.49.

$$
\begin{aligned}
-\widetilde{D}_{i, g}^{x+} \phi_{i+1, g}-\widetilde{D}_{i, g}^{x-} \phi_{i-1, g}+ & {\left[\widetilde{D}_{i, g}^{x+}+\widetilde{D}_{i, g}^{x-}+\Sigma_{r, i, g}\right] \phi_{i, g}=} \\
& \sum_{g^{\prime} \neq g}^{G} \Sigma_{s, i, g^{\prime} \rightarrow g} \phi_{i, g^{\prime}}+\frac{\chi_{i, g}}{k_{e f f}} \sum_{g^{\prime}=1}^{G} \nu \Sigma_{f, i, g} \phi_{i, g^{\prime}}
\end{aligned}
$$

\subsubsection{Diffusion Boundary Conditions}

Obviously, the boundary conditions derived in the previous chapter that depends on the angular variable can not be applied here for the neutron diffusion equation. So, it is necessary to derive new boundary conditions from the previous ones in order to eliminate de angular variable. An scheme of the cell-centered scheme is shown in fig.4.2, where the boundary conditions would be applied to cell 1 for the left boundary condition and $N$ for the right boundary condition.

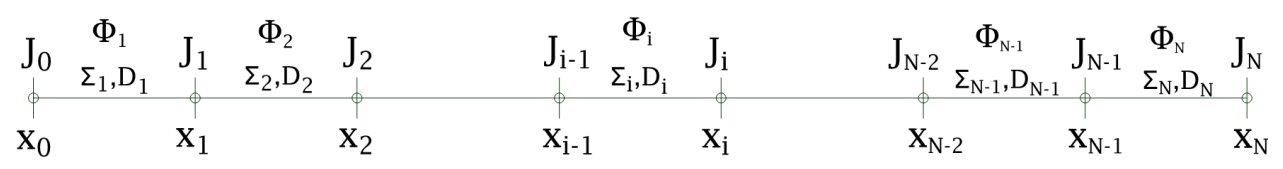

Figure 4.2: Cell-centered finite difference scheme.

\section{Reflective Condition}

The reflective condition is defined starting from the definition of the reflective boundary conditions for the neutron transport theory reviewed in the previous chapter for the left boundary condition $\left(x_{0}\right)$ :

$$
\psi\left(x_{0}, \mu\right)=\psi\left(x_{0}, \mu^{\prime}\right) \rightarrow \phi\left(x_{0}\right)+3 \mu J\left(x_{0}\right)=\phi\left(x_{0}\right)+3 \mu^{\prime} J\left(x_{0}\right)
$$

Taking into account that $\mu^{\prime}=-\mu$ in one-dimensional reflection, it can be said that the approximation to the reflective condition in transport theory is the zero net-current condition defined by:

$$
J\left(x_{0}\right)=-\left.D \frac{\partial \phi}{\partial x}\right|_{x=x_{0}}=0
$$




\section{Vacuum Condition}

Similar to the derivation of the reflective condition, it is considered that:

$$
J\left(x_{0}\right)^{i n}=\int_{0}^{1} \mu \psi\left(x_{0}, \mu\right) d \mu=0
$$

which describes that the current incoming from outside on the left boundary must be zero. If the relation $\psi(x, \mu)=\phi(x)+3 \mu J(x)$ is introduces in 4.52:

$$
J\left(x_{0}\right)^{i n}=\int_{0}^{1} \mu\left[\phi\left(x_{0}\right)+3 \mu J\left(x_{0}\right)\right] d \mu=\frac{1}{4} \phi\left(x_{0}\right)+\frac{1}{2} J\left(x_{0}\right)=0
$$

From this expression, it can be defined the Marshak boundary condition:

$$
J\left(x_{0}\right)=-\left.D \frac{\partial \phi}{\partial x}\right|_{x=x_{0}}=\frac{1}{2} \phi\left(x_{0}\right)
$$

Another commonly used expression for the vacuum boundary condition is the Mark condition. The Mark boundary condition is related with the discrete ordinates $S_{N}$ method. A simple way is to consider $S_{2}$ quadrature order with $\mu=$ $1 / \sqrt{3}$ :

$$
\left.\psi\left(x_{0}, \mu\right)\right|_{\mu=\frac{1}{\sqrt{3}}}=\phi\left(x_{0}\right)+3 \frac{1}{\sqrt{3}} J\left(x_{0}\right)=0
$$

So, similarly to the Marshak condition, the Mark conditions is:

$$
J\left(x_{0}\right)=-\left.D \frac{\partial \phi}{\partial x}\right|_{x=x_{0}}=\frac{1}{\sqrt{3}} \phi\left(x_{0}\right)
$$




\section{Albedo Condition}

With the intention of having only one expression that includes every boundary conditions, similarly to eq.3.70, the Albedo conditions are going to be defined in this section.

If the parameter $\beta$ is defined for the left boundary condition as [Hébert, 2009]:

$$
\beta=\frac{J\left(x_{0}\right)^{\text {in }}}{J\left(x_{0}\right)^{\text {out }}}
$$

And if the incoming current from the outside $J(0)^{i n}$ and the outgoing current $J(0)^{\text {out }}$ are:

$$
\begin{gathered}
J\left(x_{0}\right)^{\text {in }}=\int_{0}^{1} \mu \psi\left(x_{0}, \mu\right) d \mu=\frac{1}{4} \phi\left(x_{0}\right)+\frac{1}{2} J\left(x_{0}\right) \\
J\left(x_{0}\right)^{\text {out }}=\int_{-1}^{0}-\mu \psi\left(x_{0}, \mu\right) d \mu=\frac{1}{4} \phi\left(x_{0}\right)-\frac{1}{2} J\left(x_{0}\right)
\end{gathered}
$$

Substituting into eq.4.57:

$$
\beta=\frac{J\left(x_{0}\right)^{\text {in }}}{J\left(x_{0}\right)^{\text {out }}}=\frac{\phi\left(x_{0}\right)+2 J\left(x_{0}\right)}{\phi\left(x_{0}\right)-2 J\left(x_{0}\right)}=\frac{1-2 \alpha_{L}}{1+2 \alpha_{L}}
$$

with the parameter $\alpha_{L}$ defined for the left boundary condition as:

$$
\alpha_{L}=\frac{-J\left(x_{0}\right)}{\phi\left(x_{0}\right)}
$$

The values of $\alpha_{L}$ for the different boundary conditions are the following:

$$
\begin{aligned}
\alpha_{L}=0 & \rightarrow \text { Reflective condition: } J\left(x_{0}\right)=0, \\
\alpha_{L}=1 / 2 & \rightarrow \text { Zero incoming current (vacuum): } J\left(x_{0}\right)^{i n}=0, \\
\alpha_{L}=\infty & \rightarrow \text { Zero flux condition: } \phi\left(x_{0}\right)=0 .
\end{aligned}
$$


These boundary conditions derived for the left boundary can be extrapolated to the other boundary conditions. Next sections apply the finite difference to the boundary condition using the Albedo formulation.

\subsubsection{Application of the Left boundary condition $(i=1)$}

$$
\frac{J_{1, g}-J_{0, g}}{h_{1}}+\Sigma_{r, 1, g} \phi_{1, g}=\sum_{g^{\prime} \neq g}^{G} \Sigma_{s, 1, g^{\prime} \rightarrow g} \phi_{1, g^{\prime}}+\frac{\chi_{1, g}}{k_{e f f}} \sum_{g^{\prime}=1}^{G} \nu \Sigma_{f, 1, g^{\prime}} \phi_{1, g^{\prime}}
$$

Where:

$$
\begin{gathered}
\frac{J_{1, g}}{h_{1}}=-\widetilde{D}_{1, g}^{x+}\left[\phi_{2, g}-\phi_{1, g}\right] \\
J_{0, g}=-\alpha_{L} \cdot \phi_{1, g}^{L}=-D_{1} \frac{\phi_{1, g}-\phi_{1, g}^{L}}{h_{1} / 2} \\
\phi_{1, g}^{L}=\frac{D_{1, g} / h_{1}}{D_{1, g} / h_{1}+\alpha_{L} / 2} \phi_{1, g} \\
\frac{J_{0, g}}{h_{1}}=-2 \frac{\left(D_{1, g} / h_{1}\right)\left(\alpha_{L} / 2\right)}{\left[\left(D_{1, g} / h_{1}\right)+\left(\alpha_{L} / 2\right)\right] h_{1}} \phi_{1, g}=-\widetilde{D}_{0, g} \phi_{1, g} \\
-\widetilde{D}_{1, g}^{x+} \phi_{2, g}+\left[\widetilde{D}_{1, g}^{x+}+\widetilde{D}_{0, g}+\Sigma_{r, 1, g}\right] \phi_{1, g}=\sum_{g^{\prime} \neq g}^{G} \Sigma_{s, 1, g^{\prime} \rightarrow g} \phi_{1, g^{\prime}}+\frac{\chi_{1, g}}{k_{e f f}} \sum_{g^{\prime}=1}^{G} \nu \Sigma_{f, 1, g^{\prime}} \phi_{1, g^{\prime}}
\end{gathered}
$$

\subsubsection{Application of the Right boundary condition $(i=N)$}

$$
\frac{J_{N, g}-J_{N-1, g}}{h_{N}}+\Sigma_{r, N, g} \phi_{N, g}=\sum_{g^{\prime} \neq g}^{G} \Sigma_{s, N, g^{\prime} \rightarrow g} \phi_{N, g^{\prime}}+\frac{\chi_{N, g}}{k_{e f f}} \sum_{g^{\prime}=1}^{G} \nu \Sigma_{f, N, g} \phi_{N, g^{\prime}}
$$

Where:

$$
\frac{J_{N-1, g}}{h_{1}}=-\widetilde{D}_{N, g}^{x+}\left[\phi_{N, g}-\phi_{N-1, g}\right]
$$




$$
\begin{gathered}
J_{N, g}=-\alpha_{R} \cdot \phi_{N, g}^{R}=-D_{N, g} \frac{\phi_{N, g}-\phi_{N-1, g}^{R}}{h_{N} / 2} \\
\frac{J_{N, g}}{h_{N}}=2 \frac{\left(D_{N, g} / h_{N}\right)\left(\alpha_{R} / 2\right)}{\left[\left(D_{N, g} / h_{N}\right)+\left(\alpha_{R} / 2\right)\right] h_{N}} \phi_{N, g}=\widetilde{D}_{N, g} \phi_{N, g} \\
-\widetilde{D}_{N, g}^{x-} \phi_{N-1, g}+\left[\widetilde{D}_{N, g}^{x-}+\widetilde{D}_{N, g}+\Sigma_{r, N, g}\right] \phi_{N, g}= \\
\sum_{g^{\prime} \neq g}^{G} \Sigma_{s, N, g^{\prime} \rightarrow g} \phi_{N, g^{\prime}}+\frac{\chi_{N, g}}{k_{e f f}} \sum_{g^{\prime}=1}^{G} \nu \Sigma_{f, N, g^{\prime}} \phi_{N, g^{\prime}}
\end{gathered}
$$

\subsection{D Finite Difference Diffusion Equation using Cell-Centered Scheme}

This section describes the formulation of the 2D finite difference diffusion equation using the cell-centered scheme and its boundary conditions for each boundary point in a Cartesian geometry. The size of the cartesian geometry is $1 \leq i \leq N$ and $1 \leq j \leq M$ and distance in each interval in axis $x$ and $y$ are $h_{i}$ and $k_{j}$ respectively. This section also explains the boundary formulation for edges and corners. Following the same procedure that in the 1D diffusion equation, one can obtain:

$$
\begin{gathered}
-\widetilde{D}_{i, j, g}^{x+} \phi_{i+1, j, g}-\widetilde{D}_{i, j, g}^{y+} \phi_{i, j+1, g}-\widetilde{D}_{i, j, g}^{y-} \phi_{i, j-1, g}-\widetilde{D}_{i, j, g}^{x-} \phi_{i-1, j, g}+ \\
{\left[\widetilde{D}_{i, j, g}^{x+}+\widetilde{D}_{i, j, g}^{y+}+\widetilde{D}_{i, j, g}^{y-}+\widetilde{D}_{i, j, g}^{x-}+\Sigma_{r, i, j, g}\right] \phi_{i, j, g}=} \\
\sum_{g^{\prime} \neq g}^{G} \Sigma_{s, i, j, g^{\prime} \rightarrow g} \phi_{i, j, g^{\prime}}+\frac{\chi_{i, j, g}}{k_{e f f}} \sum_{g^{\prime}=1}^{G} \nu \Sigma_{f, i, j, g^{\prime}} \phi_{i, j, g^{\prime}}
\end{gathered}
$$

Where:

$$
\begin{aligned}
& \widetilde{D}_{i, j, g}^{x+}=\frac{2 D_{i, j, g} D_{i+1, j, g}}{\left(D_{i, j, g} h_{i+1}+D_{i+1, j, g} h_{i}\right) h_{i}} \\
& \widetilde{D}_{i, j, g}^{x-}=\frac{2 D_{i-1, j, g} D_{i, j, g}}{\left(D_{i-1, j, g} h_{i}+D_{i, j, g} h_{i-1}\right) h_{i}}
\end{aligned}
$$




$$
\begin{aligned}
\widetilde{D}_{i, j, g}^{y+} & =\frac{2 D_{i, j, g} D_{i, j+1, g}}{\left(D_{i, j, g} h_{i+1}+D_{i, j+1, g} h_{i}\right) h_{i}} \\
\widetilde{D}_{i, j, g}^{y-} & =\frac{2 D_{i, j-1, g} D_{i, j, g}}{\left(D_{i, j-1, g} h_{i}+D_{i, j, g} h_{i-1}\right) h_{i}}
\end{aligned}
$$

4.4.1 Application of the Left Boundary Condition $(i=1, j=2: M)$

$$
\begin{gathered}
-\widetilde{D}_{1, j, g}^{x+} \phi_{2, j, g}-\widetilde{D}_{1, j, g}^{y+} \phi_{1, j+1, g}-\widetilde{D}_{1, j, g}^{y-} \phi_{1, j-1, g}+ \\
{\left[\widetilde{D}_{1, j, g}^{x+}+\widetilde{D}_{0, j, g}+\widetilde{D}_{1, j, g}^{y+}+\widetilde{D}_{1, j, g}^{y-}+\Sigma_{r, 1, j, g}\right] \phi_{1, j, g}=} \\
\sum_{g^{\prime} \neq g}^{G} \Sigma_{s, 1, j, g^{\prime} \rightarrow g} \phi_{1, j, g^{\prime}}+\frac{\chi_{1, j, g}}{k_{e f f}} \sum_{g^{\prime}=1}^{G} \nu \Sigma_{f, 1, j, g^{\prime}} \phi_{1, j, g^{\prime}}
\end{gathered}
$$

Where:

$$
\widetilde{D}_{0, j, g}=2 \frac{\left(D_{1, j, g} / h_{1}\right)\left(\alpha_{L} / 2\right)}{\left(D_{1, j, g} / h_{1}+\alpha_{L} / 2\right) h_{1}}
$$

4.4.2 Application of the Right Boundary Condition $(i=N, j=2: M)$

$$
\begin{aligned}
&-\widetilde{D}_{N, j, g}^{x-} \phi_{N-1, j, g}- \widetilde{D}_{N, j, g}^{y+} \phi_{N, j+1, g}-\widetilde{D}_{N, j, g}^{y-} \phi_{N, j-1, g}+ \\
& {\left[\widetilde{D}_{N, j, g}+\widetilde{D}_{N, j, g}^{x-}+\widetilde{D}_{N, j, g}^{y+}+\widetilde{D}_{N, j, g}^{y-}+\Sigma_{r, N, j, g}\right] \phi_{N, j, g}=} \\
& \sum_{g^{\prime} \neq g}^{G} \Sigma_{s, N, j, g^{\prime} \rightarrow g} \phi_{N, j, g^{\prime}}+\frac{\chi_{N, j, g}}{k_{e f f}} \sum_{g^{\prime}=1}^{G} \nu \Sigma_{f, N, j, g^{\prime}} \phi_{N, j, g^{\prime}}
\end{aligned}
$$

Where:

$$
\widetilde{D}_{N, j, g}=2 \frac{\left(D_{N, j, g} / h_{N}\right)\left(\alpha_{L} / 2\right)}{\left(D_{N, j, g} / h_{N}+\alpha_{L} / 2\right) h_{N}}
$$


4.4.3 Application of the Bottom Boundary Condition $(i=2: N, j=1)$

$$
\begin{gathered}
-\widetilde{D}_{i, 1, g}^{x-} \phi_{i-1,1, g}-\widetilde{D}_{i, 1, g}^{x+} \phi_{i+1,1, g}-\widetilde{D}_{i, 1, g}^{y+} \phi_{i, 2, g}+\left[\widetilde{D}_{i, 1, g}^{y+}+\widetilde{D}_{i, 0, g}+\widetilde{D}_{i, 1, g}^{x-}+\widetilde{D}_{i, 1, g}^{x+}+\Sigma_{r, i, 1, g}\right] \phi_{i, 1, g}= \\
\sum_{g^{\prime} \neq g}^{G} \Sigma_{s, i, 1, g^{\prime} \rightarrow g} \phi_{i, 1, g^{\prime}}+\frac{\chi_{i, 1, g}}{k_{e f f}} \sum_{g^{\prime}=1}^{G} \nu \Sigma_{f, i, 1, g^{\prime}} \phi_{i, 1, g^{\prime}}
\end{gathered}
$$

Where:

$$
\widetilde{D}_{i, 0, g}=2 \frac{\left(D_{i, 1, g} / k_{1}\right)\left(\alpha_{B} / 2\right)}{\left(D_{i, 1, g} / k_{1}+\alpha_{B} / 2\right) k_{1}}
$$

4.4.4 Application of the Top Boundary Condition $(i=2: N, j=M)$

$$
\begin{gathered}
-\widetilde{D}_{i, M, g}^{x-} \phi_{i-1, M, g}-\widetilde{D}_{i, M, g}^{x+} \phi_{i+1, M, g}-\widetilde{D}_{i, M, g}^{y-} \phi_{i, M-1, g}+ \\
{\left[\widetilde{D}_{i, M, g}^{y-}+\widetilde{D}_{i, M, g}+\widetilde{D}_{i, M, g}^{x-}+\widetilde{D}_{i, M, g}^{x+}+\Sigma_{r, i, M, g}\right] \phi_{i, M, g}=} \\
\sum_{g^{\prime} \neq g}^{G} \Sigma_{s, i, M, g^{\prime} \rightarrow g} \phi_{i, M, g^{\prime}}+\frac{\chi_{i, M, g}}{k_{e f f}} \sum_{g^{\prime}=1}^{G} \nu \Sigma_{f, i, M, g^{\prime}} \phi_{i, M, g^{\prime}}
\end{gathered}
$$

Where:

$$
\widetilde{D}_{i, M, g}=2 \frac{\left(D_{i, M, g} / k_{M}\right)\left(\alpha_{T} / 2\right)}{\left(D_{i, M, g} / k_{M}+\alpha_{T} / 2\right) k_{M}}
$$

\subsubsection{Application of the Left-Bottom corner boundary condition}

$$
\begin{aligned}
& (i=1, j=1) \\
& -\widetilde{D}_{1,1, g}^{x+} \phi_{2,1, g}-\widetilde{D}_{1,1, g}^{y+} \phi_{1,2, g}+ \\
& {\left[\widetilde{D}_{0,1, g}+\widetilde{D}_{1,0, g}+\widetilde{D}_{1,1, g}^{x+}+\widetilde{D}_{1,1, g}^{y+}+\Sigma_{r, 1,1, g}\right] \phi_{1,1, g}=} \\
& \sum_{g^{\prime} \neq g}^{G} \nu \Sigma_{s, 1,1, g^{\prime} \rightarrow g} \phi_{1,1, g^{\prime}}+\frac{\chi_{1,1, g}}{k_{e f f}} \sum_{g^{\prime}=1}^{G} \Sigma_{f, 1,1, g^{\prime}} \phi_{1,1, g^{\prime}}
\end{aligned}
$$

Where: 


$$
\begin{aligned}
& \widetilde{D}_{0,1, g}=2 \frac{\left(D_{1,1, g} / h_{1}\right)\left(\alpha_{L} / 2\right)}{\left(D_{1,1, g} / h_{1}+\alpha_{L} / 2\right) h_{1}} \\
& \widetilde{D}_{1,0, g}=2 \frac{\left(D_{1,1, g} / k_{1}\right)\left(\alpha_{B} / 2\right)}{\left(D_{1,1, g} / k_{1}+\alpha_{B} / 2\right) k_{1}}
\end{aligned}
$$

4.4.6 Application of the Right-Top corner boundary condition $(i=N, j=M)$

$$
\begin{aligned}
& -\widetilde{D}_{N, M, g}^{x-} \phi_{N-1, M, g}-\widetilde{D}_{N, M, g}^{y-} \phi_{N, M-1, g}+ \\
& {\left[\widetilde{D}_{N, M, g}^{x}+\widetilde{D}_{N, M, g}^{y}+\widetilde{D}_{N, M, g}^{y+}+\widetilde{D}_{N, M, g}^{y-}+\Sigma_{r, N, M, g}\right] \phi_{N, M, g}=} \\
& \sum_{g^{\prime} \neq g}^{G} \Sigma_{s, N, M, g^{\prime} \rightarrow g} \phi_{N, M, g^{\prime}}+\frac{\chi_{N, M, g}}{k_{e f f}} \sum_{g^{\prime}=1}^{G} \nu \Sigma_{f, N, M, g^{\prime}} \phi_{N, M, g^{\prime}}
\end{aligned}
$$

Where:

$$
\begin{aligned}
& \widetilde{D}_{N, M, g}^{x}=2 \frac{\left(D_{N, M, g} / h_{N}\right)\left(\alpha_{L} / 2\right)}{\left(D_{N, M, g} / h_{N}+\alpha_{L} / 2\right) h_{N}} \\
& \widetilde{D}_{N, M, g}^{y}=2 \frac{\left(D_{N, M, g} / k_{M}\right)\left(\alpha_{T} / 2\right)}{\left(D_{N, M, g} / k_{M}+\alpha_{T} / 2\right) k_{M}}
\end{aligned}
$$

4.4.7 Application of the Left-Top corner boundary condition $(i=1, j=M)$

$$
\begin{aligned}
&-\widetilde{D}_{1, M, g}^{x+} \phi_{2, M, g}- \widetilde{D}_{1, M, g}^{y-} \phi_{1, M-1, g}+ \\
& {\left[\widetilde{D}_{0, M, g}+\widetilde{D}_{1, M, g}+\widetilde{D}_{1, M, g}^{x+}+\widetilde{D}_{1, M, g}^{y-}+\Sigma_{r, 1, M, g}\right] \phi_{1, M, g}=} \\
& \sum_{g^{\prime} \neq g}^{G} \Sigma_{s, 1, M, g^{\prime} \rightarrow g} \phi_{1, M, g^{\prime}}+\frac{\chi_{1, M, g}}{k_{e f f}} \sum_{g^{\prime}=1}^{G} \nu \Sigma_{f, 1, M, g^{\prime}} \phi_{1, M, g^{\prime}}
\end{aligned}
$$

Where: 


$$
\begin{gathered}
\widetilde{D}_{0, M, g}=2 \frac{\left(D_{1, M, g} / h_{1}\right)\left(\alpha_{L} / 2\right)}{\left(D_{1, M, g} / h_{1}+\alpha_{L} / 2\right) h_{1}} \\
\widetilde{D}_{1, M, g}=2 \frac{\left(D_{1, M, g} / k_{M}\right)\left(\alpha_{T} / 2\right)}{\left(D_{1, M, g} / k_{M}+\alpha_{T} / 2\right) k_{M}}
\end{gathered}
$$

\subsubsection{Application of the Right-Bottom corner boundary condition}

$$
\begin{aligned}
& (i=N, j=1) \\
& -\widetilde{D}_{N, 1, g}^{x-} \phi_{N-1,1, g}-\widetilde{D}_{N, 1, g}^{y+} \phi_{N, 2, g}+ \\
& {\left[\widetilde{D}_{N, 1, g}+\widetilde{D}_{N, 0, g}+\widetilde{D}_{N, 1, g}^{x-}+\widetilde{D}_{N, 1, g}^{y+}+\Sigma_{r, N, 1, g}\right] \phi_{N, 1, g}=} \\
& \sum_{g^{\prime} \neq g}^{G} \Sigma_{s, N, 1, g^{\prime} \rightarrow g} \phi_{N, 1, g^{\prime}}+\frac{\chi_{N, 1, g}}{k_{e f f}} \sum_{g^{\prime}=1}^{G} \nu \Sigma_{f, N, 1, g^{\prime}} \phi_{N, 1, g^{\prime}}
\end{aligned}
$$

Where:

$$
\begin{gathered}
\widetilde{D}_{N, 1, g}=2 \frac{\left(D_{N, 1, g} / h_{N}\right)\left(\alpha_{L} / 2\right)}{\left(D_{N, 1, g} / h_{N}+\alpha_{L} / 2\right) h_{N}} \\
\widetilde{D}_{N, 0, g}=2 \frac{\left(D_{N, 1, g} / k_{1}\right)\left(\alpha_{B} / 2\right)}{\left(D_{N, 1, g} / k_{1}+\alpha_{B} / 2\right) k_{1}}
\end{gathered}
$$




\subsection{D Finite Difference Diffusion Equation using Edge-Centered Scheme}

This section presents the detailed formulation derived to solve the one-dimensional finite difference diffusion equation, but in this case, an edge-centered scheme was performed. Returning to the eq.4.35:

$$
\frac{\partial}{\partial x} J(x)+\Sigma_{r, g}(x) \phi_{g}(x)=\sum_{g^{\prime} \neq g}^{G} \Sigma_{s, g^{\prime} \rightarrow g}(x) \phi_{g^{\prime}}(x)+\frac{\chi_{g}(x)}{k_{e f f}} \sum_{g^{\prime}=1}^{G} \nu \Sigma_{f, g^{\prime}}(x) \phi_{g^{\prime}}(x)
$$

and using finite difference edge-centered scheme approximation according to the fig.4.3 and fig.4. 4 it is obtained eq.4.98:

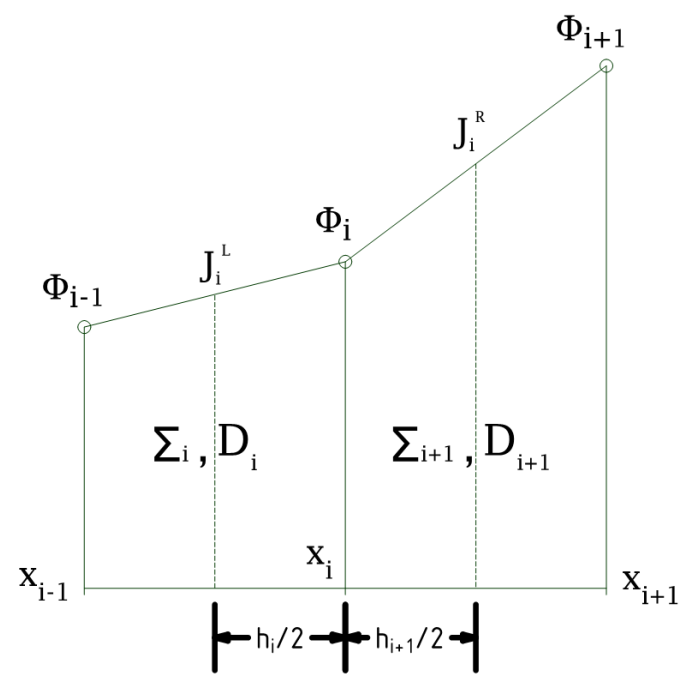

Figure 4.3: Finite difference edge-centered scheme.

$$
\begin{array}{r}
J_{g, i}^{R}-J_{g, i}^{L}+\int_{x_{i-1}+h_{i} / 2}^{x_{i}+h_{i+1} / 2} \Sigma_{r, g}(x) \phi_{g}(x) d x=\int_{x_{i-1}+h_{i} / 2}^{x_{i}+h_{i+1} / 2} \sum_{g^{\prime} \neq g}^{G} \Sigma_{s, g^{\prime} \rightarrow g}(x) \phi_{g^{\prime}}(x) d x+ \\
\frac{\chi_{g}(x)}{k_{e f f}} \int_{x_{i-1}+h_{i} / 2}^{x_{i}+h_{i+1} / 2} \sum_{g^{\prime}=1}^{G} \nu \Sigma_{f, g^{\prime}}(x) \phi_{g^{\prime}}(x) d x
\end{array}
$$




$$
\begin{gathered}
\int_{x_{i-1}+h_{i} / 2}^{x_{i}+h_{i+1} / 2} \Sigma_{r, g}(x) \phi_{g}(x) d x \approx \phi_{g}\left(x_{i}\right) \int_{x_{i-1}+h_{i} / 2}^{x_{i}+h_{i+1} / 2} \Sigma_{r, g}(x) d x= \\
\frac{1}{2}\left(h_{i} \Sigma_{r, g, i}+h_{i+1} \Sigma_{r, g, i+1}\right) \phi_{g, i}=\bar{\Sigma}_{r, g, i} \phi_{g, i}, \quad \text { (4.99) } \\
\int_{x_{i-1}+h_{i} / 2}^{x_{i}+h_{i+1} / 2} \sum_{g^{\prime} \neq g} \Sigma_{s, g^{\prime} \rightarrow g}(x) \phi_{g^{\prime}}(x) d x \approx \sum_{g^{\prime} \neq g}^{G} \frac{1}{2}\left(h_{i} \Sigma_{s, g^{\prime} \rightarrow g, i}+h_{i+1} \Sigma_{s, g^{\prime} \rightarrow g, i+1}\right) \phi_{g^{\prime}, i} \\
=\sum_{g^{\prime} \neq g}^{G} \bar{\Sigma}_{s, g^{\prime} \rightarrow g, i} \phi_{g^{\prime}, i}, \quad \text { (4.100) } \\
\int_{x_{i-1}+h_{i} / 2}^{x_{i}+h_{i+1} / 2} \sum_{g^{\prime}} \nu \Sigma_{f, g^{\prime}}(x) \phi_{g^{\prime}}(x) d x \approx \sum_{g^{\prime}=1}^{G} \frac{1}{2}\left(h_{i} \nu \Sigma_{f, g^{\prime}, i}+h_{i+1} \nu \Sigma_{f, g^{\prime}, i+1}\right) \phi_{i, g^{\prime}} \\
=\sum_{g^{\prime}=1}^{G} \frac{\Sigma_{f, g^{\prime}, i} \phi_{g^{\prime}, i}, \quad(4.101)}{}
\end{gathered}
$$

Substituting eqs.4.99, 4.100 and 4.101 into eq.4.98:

$$
J_{g, i}^{R}-J_{g, i}^{L}+\bar{\Sigma}_{r, g, i} \phi_{g, i}=\sum_{g^{\prime} \neq g}^{G} \bar{\Sigma}_{s, g^{\prime} \rightarrow g, i} \phi_{g^{\prime}, i}+\frac{\chi_{g, i}}{k_{e f f}} \sum_{g^{\prime}=1}^{G} \bar{\nu}_{f, g^{\prime}, i} \phi_{g^{\prime}, i}
$$

Defining the currents as:

$$
\begin{gathered}
J_{g, i}^{R}(x)=-D_{g, i}(x) \frac{\partial \phi_{g}(x)}{\partial x}, \\
J_{g, i}^{R}=-D_{g, i+1} \frac{\phi_{g, i+1}-\phi_{g, i}}{h_{i+1}}, \\
J_{g, i}^{L}=-D_{g, i} \frac{\phi_{g, i}-\phi_{g, i-1}}{h_{i}} .
\end{gathered}
$$

Finally, Eq.4.102 is rewritten as a discretized mesh balance equation as follows: 


$$
\begin{gathered}
{\left[\frac{D_{g, i+1}}{h_{i+1}}+\frac{D_{g, i}}{h_{i}}+\bar{\Sigma}_{r, g, i}\right] \phi_{g, i}-\frac{D_{g, i+1}}{h_{i+1}} \phi_{g, i+1}-\frac{D_{g, i}}{h_{i}} \phi_{g, i-1}=} \\
\sum_{g^{\prime} \neq g} \bar{\Sigma}_{s, g^{\prime} \rightarrow g, i} \phi_{g^{\prime}, i}+\frac{\chi_{g, i}}{k_{e f f}} \sum_{g^{\prime}} \overline{\nu \Sigma}_{f, g^{\prime}, i} \phi_{g^{\prime}, i} .
\end{gathered}
$$

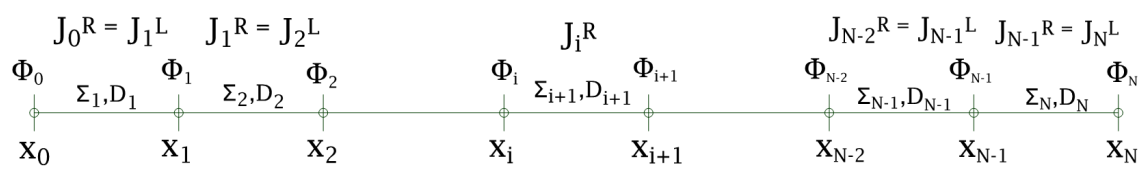

Figure 4.4: Edge-centered Scheme.

Application of the Left boundary condition $(i=0)$

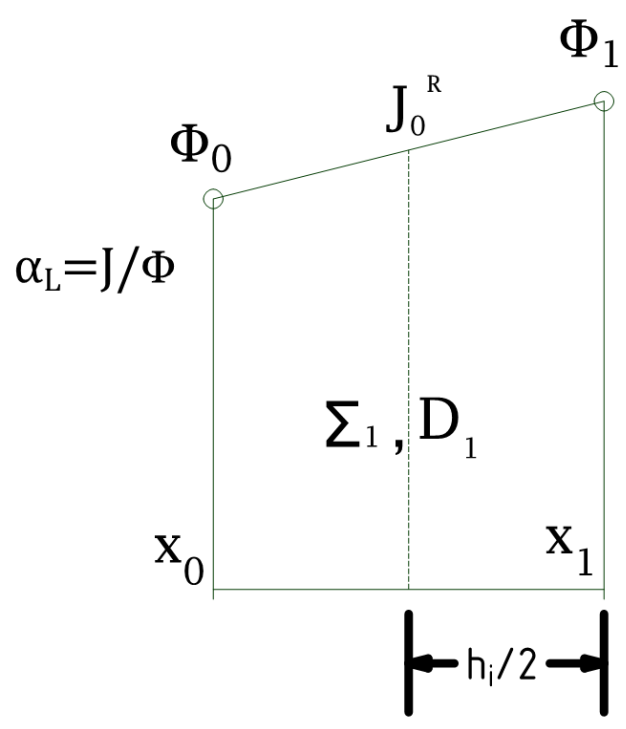

Figure 4.5: Left boundary conditions edge-centered scheme. 


$$
J_{g, 0}^{R}-J_{g, 0}+\bar{\Sigma}_{r, g, 0} \phi_{g, 0}=\sum_{g^{\prime} \neq g}^{G} \bar{\Sigma}_{s, g^{\prime} \rightarrow g, 0} \phi_{g^{\prime}, 0}^{0}+\frac{\chi_{g, 0}}{k_{e f f}} \sum_{g^{\prime}=1}^{G} \bar{\nu}_{f, g^{\prime}, 0} \phi_{g^{\prime}, 0} .
$$

with:

$$
\begin{gathered}
J_{g, 0}^{R}=-D_{g, 1} \frac{\phi_{g, 1}-\phi_{g, 0}}{h_{1} / 2}, \\
J_{g, 0}=-\alpha_{L} \phi_{g, 0},
\end{gathered}
$$

So, the application of the left boundary condition to the finite difference form of the diffusion equation gives:

$$
\left[\frac{D_{g, 1}}{h_{1}}+\alpha_{L}+\bar{\Sigma}_{r, g, 0}\right] \phi_{g, 0}-\frac{D_{g, 1}}{h_{1}} \phi_{g, 1}=\sum_{g^{\prime} \neq g}^{G} \bar{\Sigma}_{s, g^{\prime} \rightarrow g, 0} \phi_{g^{\prime}, 0}+\frac{\chi_{g, 0}}{k_{e f f}} \sum_{g^{\prime}=1}^{G} \bar{\nu}_{f, g^{\prime}, 0} \phi_{g^{\prime}, 0} .
$$

Application of the Right boundary condition $(i=N)$

By using the same procedure for the right boundary one can obtain:

$$
\begin{aligned}
{\left[\frac{D_{g, N}}{h_{N}}+\alpha_{R}+\bar{\Sigma}_{r, g, N}\right] } & \phi_{g, N}-\frac{D_{g, N}}{h_{N}} \phi_{g, N-1}= \\
& \sum_{g^{\prime} \neq g}^{G} \bar{\Sigma}_{s, g^{\prime} \rightarrow g, N} \phi_{g^{\prime}, N}+\frac{\chi_{g, N}}{k_{e f f}} \sum_{g^{\prime}=1}^{G} \overline{\nu \Sigma}_{f, g^{\prime}, N} \phi_{g^{\prime}, N} .
\end{aligned}
$$

To derive the multidimensional formulation one can derive it from the one dimensional formulation stated in this section. This formulation can be easily extrapolated to two-dimensional and three-dimensional problems. Multidimensional formulations have not been presented in this work for brevity. The numerical results in next section includes the solution to multidimensional problems. 


\subsection{Eigenvalue problem}

As was mentioned earlier, the methods developed in this work uses the KrylovSchur algorithm embedded into the SLEPc library to solve the eigenvalue problem. This is the states of the art software library for solving eigenproblems of large and sparse matrices Hernandez et al. [2005]. SLEPc needs PETSc to be completely functional and to be able of calculating the solution of eigenvalue problems. PETSc includes matrix operations as well as the solution of linear systems Balay et al. [2019]. However, some improvements were carried out with respect to the previous chapter. In the previous chapter, the shape of the matrices did not allow the use of iterative solvers. For this reason, were used direct solvers, such as the LU factorization. A new matrices rearrangement was tested to allow using iterative methods. Several iterative methods to solve the system of linear equations were tested for FDM $S_{N}$ and FDM Diffusion solvers. Finally the method implemented for FDM $S_{N}$ was a slight variant of the Enhanced BiCGStab(L) algorithm (BCGSL) Sleijpen and Van der Vorst [1995] using as a preconditioner the point block Jacobi preconditioner (BJACOBI). The method implemented for FDM of the Diffusion equation was the generalized minimal residual method (GMRES) Saad and Schultz [1986] using as a preconditioner an the ILU factorization.

The following figs.4.7-4.22 shows the new form of the matrix $A$ for different cases of the $S_{N}$ method and Diffusion method. The rearrangement for the $S_{N}$ method was performed as follows:

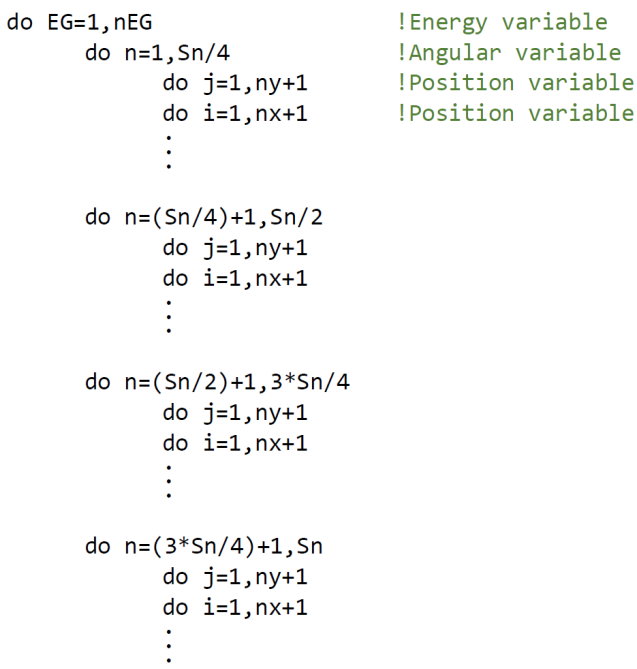

Figure 4.6: New two dimensional ordering scheme of the matrix $A$ for $S_{N}$ method. 


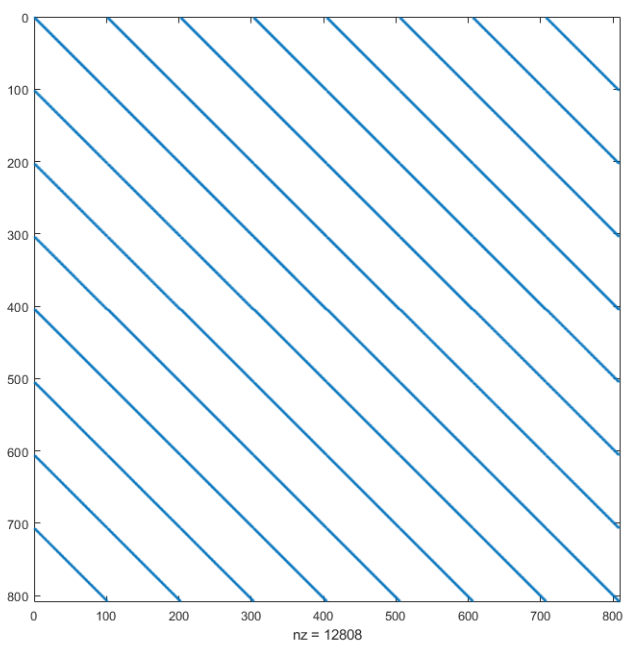

Figure 4.7: Matrix $A, 1 \mathrm{D}-1 \mathrm{EG}-S_{8}-100$ cells.

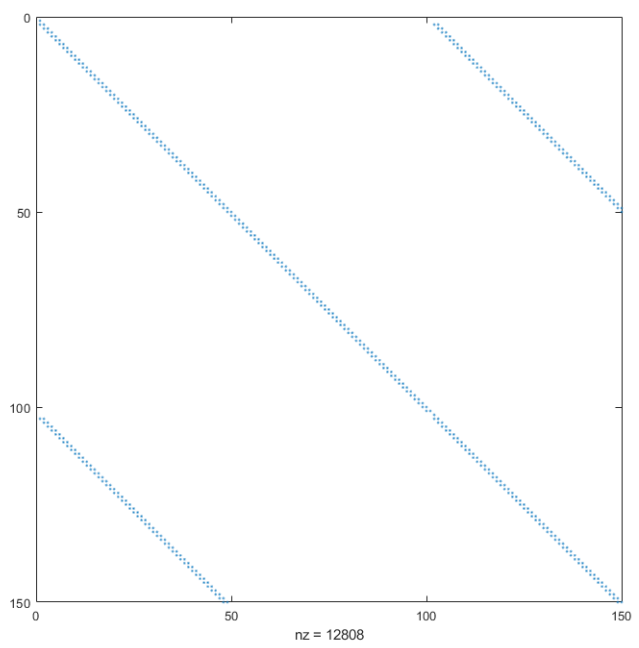

Figure 4.8: Matrix $A, 1 \mathrm{D}-1 \mathrm{EG}-S_{8}-100$ cells-zoom. 


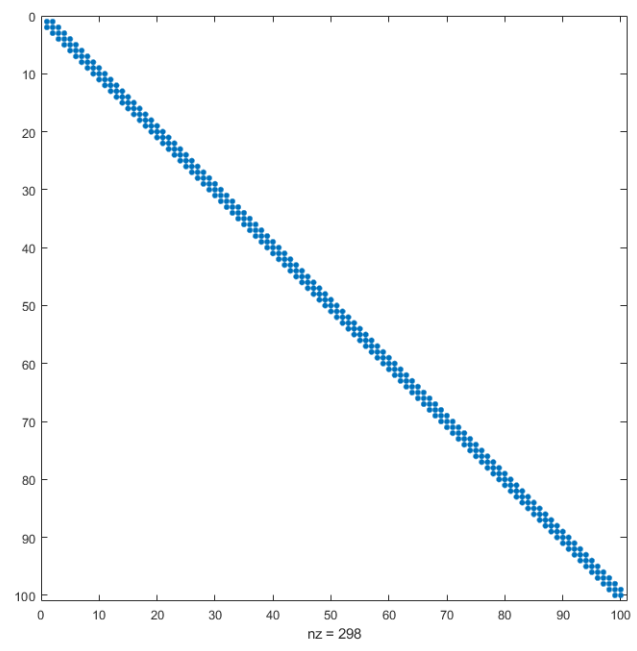

Figure 4.9: Matrix $A, 1 \mathrm{D}-1 \mathrm{EG}-S P_{1}$ (Diffusion)-100 cells.

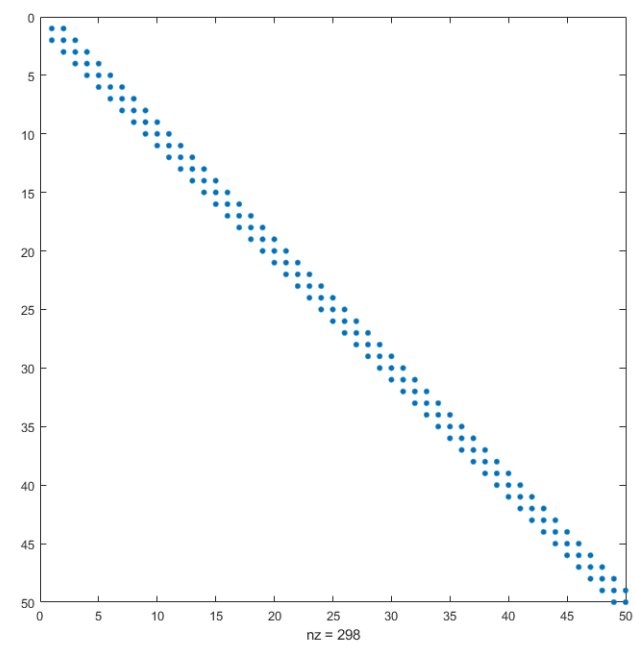

Figure 4.10: Matrix $A$, 1D-1EG- $S P_{1}$ (Diffusion)-100 cells-zoom. 


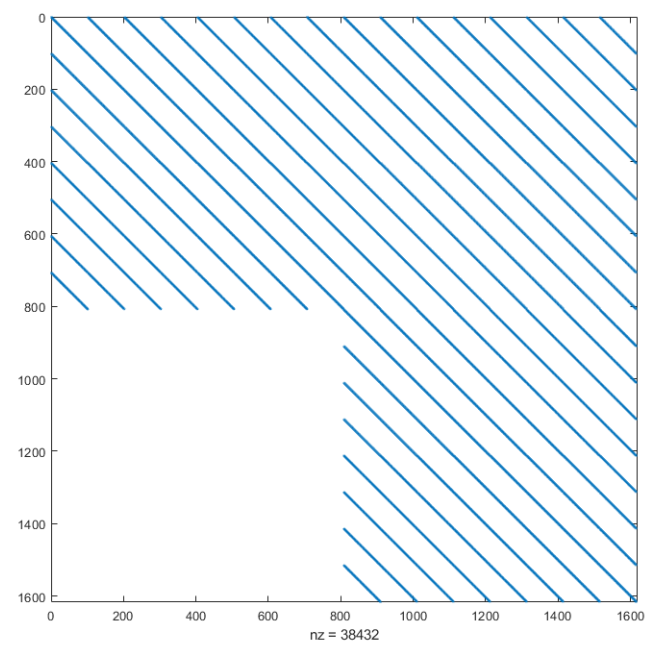

Figure 4.11: Matrix $A, 1 \mathrm{D}-2 \mathrm{EG}-S_{8}-100$ cells-no upscattering.

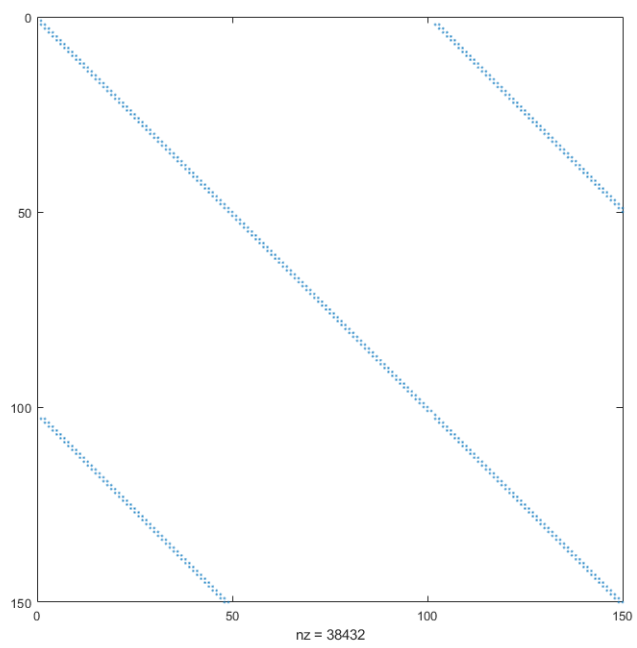

Figure 4.12: Matrix $A, 1 \mathrm{D}-2 \mathrm{EG}-S_{8}-100$ cells-no upscattering -zoom. 


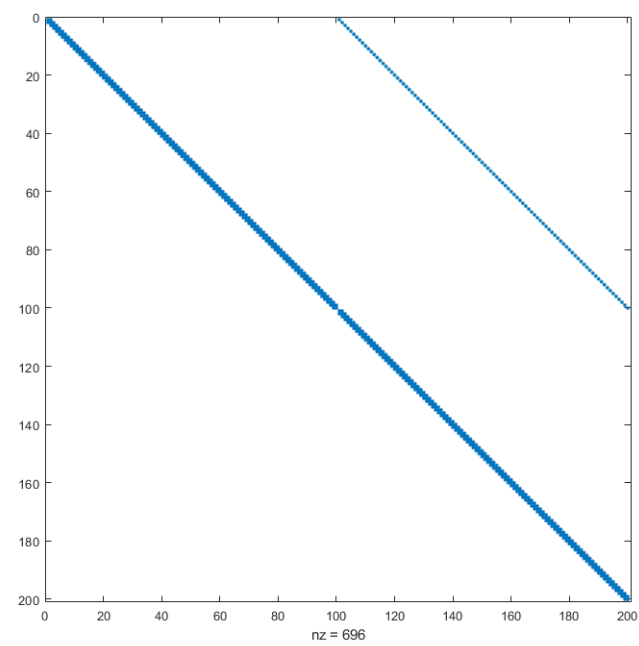

Figure 4.13: Matrix $A, 1 \mathrm{D}-2 \mathrm{EG}-S P_{1}$ (Diffusion)-100 cells-no upscattering.

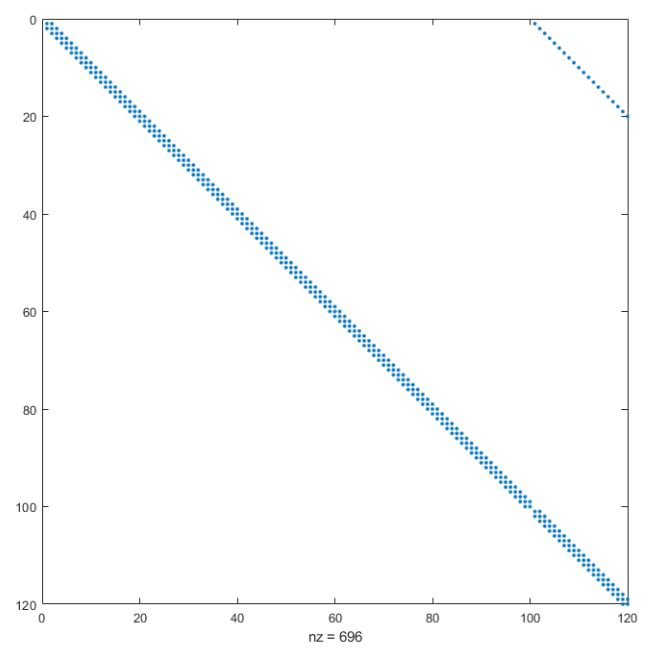

Figure 4.14: Matrix $A, 1 D-2 E G-S P_{1}$ (Diffusion)-100 cells-no upscattering -zoom. 


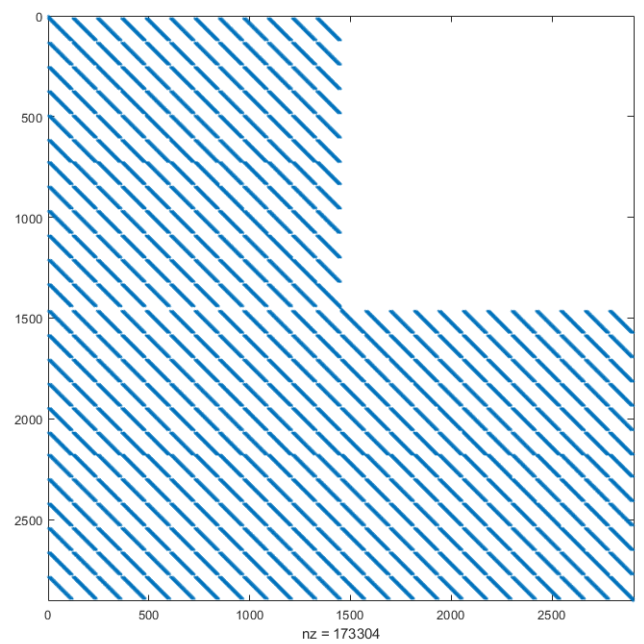

Figure 4.15: Matrix $A, 2 \mathrm{D}-2 \mathrm{EG}-S_{4}-10 \mathrm{x} 10$ cells-no upscattering.

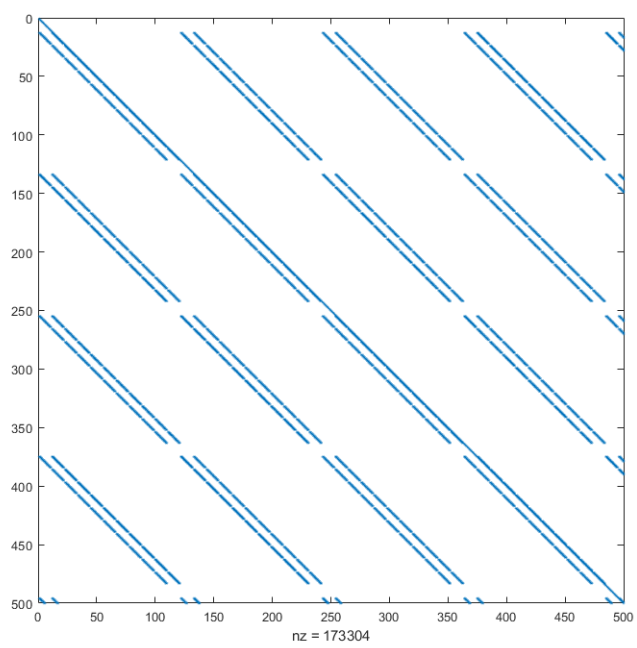

Figure 4.16: Matrix $A$, 2D-2EG- $S_{4}-10 x 10$ cells-no upscattering-zoom1. 


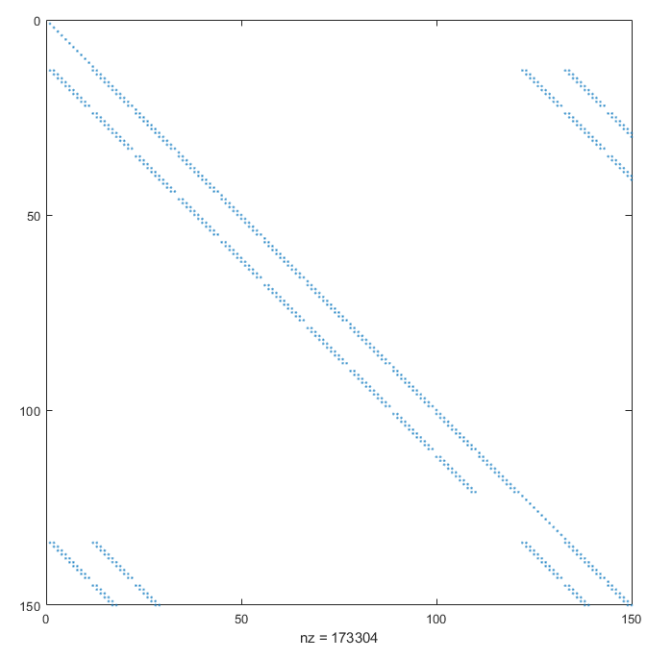

Figure 4.17: Matrix $A$, 2D-2EG- $S_{4}-10 x 10$ cells-no upscattering-zoom2.

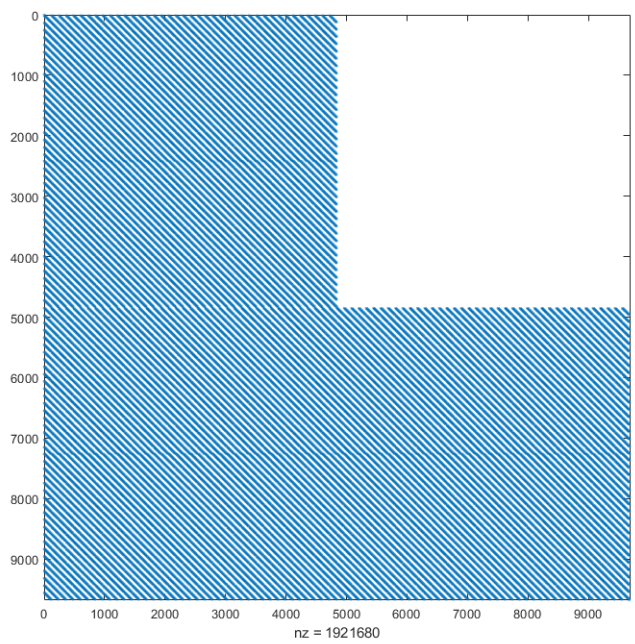

Figure 4.18: Matrix $A, 2 \mathrm{D}-2 \mathrm{EG}-S_{8}-10 \mathrm{x} 10$ cells-no upscattering. 


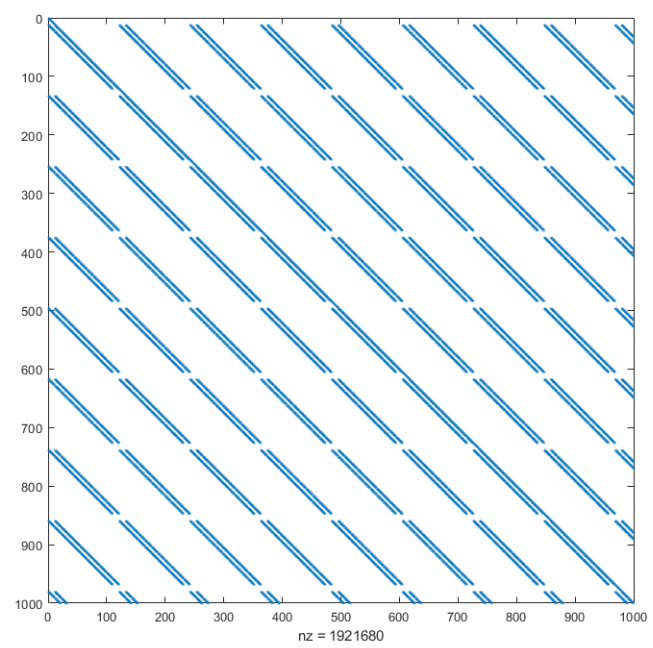

Figure 4.19: Matrix $A, 2 \mathrm{D}-2 \mathrm{EG}-S_{8}-10 x 10$ cells-no upscattering-zoom1.

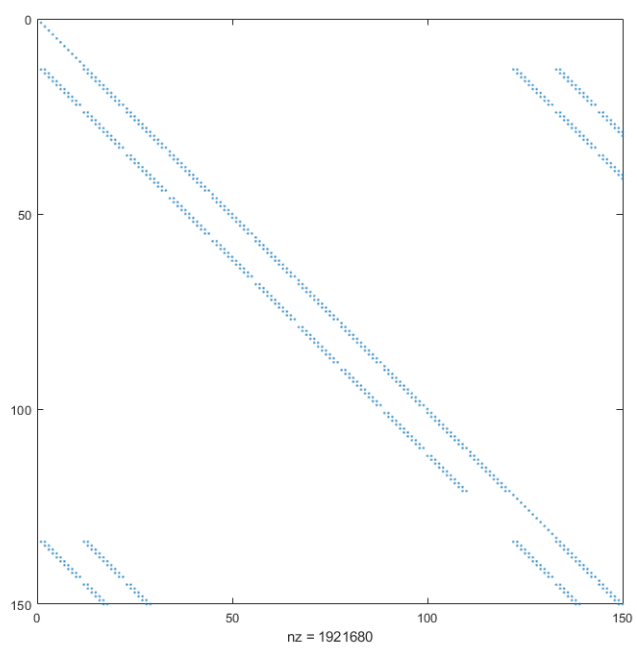

Figure 4.20: Matrix $A, 2 \mathrm{D}-2 \mathrm{EG}-S_{8}-10 \mathrm{x} 10$ cells-no upscattering-zoom2. 


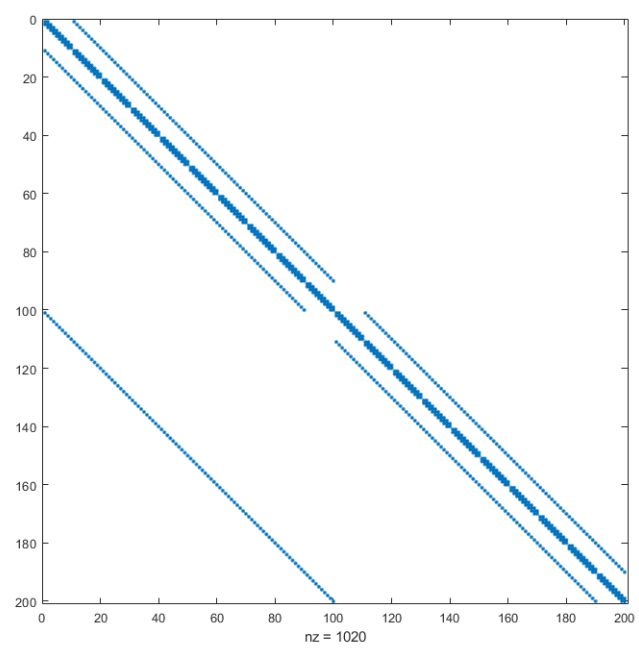

Figure 4.21: Matrix $A, 2 \mathrm{D}-2 \mathrm{EG}-S_{8}-10 \mathrm{x} 10$ cells-no upscattering.

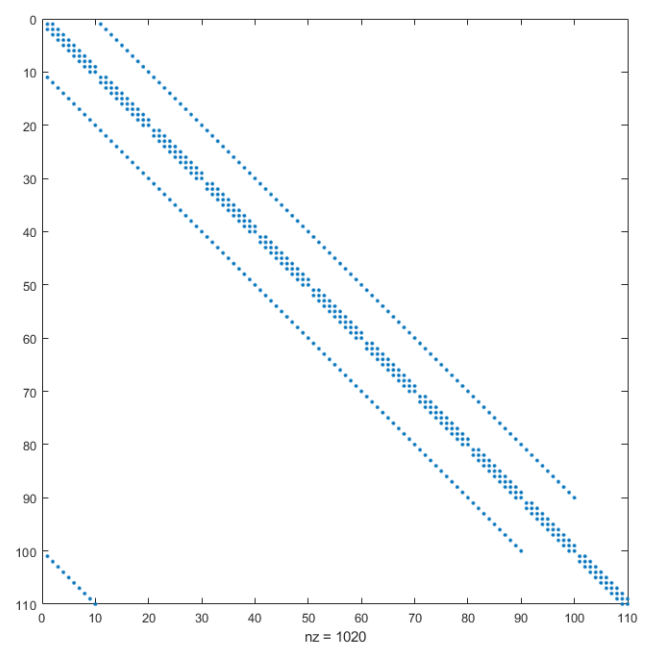

Figure 4.22: Matrix $A, 2 \mathrm{D}-2 \mathrm{EG}-S_{8}-10 \mathrm{x} 10$ cells-no upscattering-zoom. 


\subsection{Numerical Results}

This section shows a comparison between the results calculated with the methods explained in the previous chapter for discrete ordinates equations [Morató et al., 2020] and the methods explained for the diffusion equation. The improved discrete ordinates method and the diffusion algorithms explained in this chapter where implemented in a FORTRAN programming language. This code was called ValTran (Valencian Transport), which use the same input to solve problems with both methods $S_{N}$ and diffusion.

Three 1D and four 2D benchmarks were selected to test the methods. The 1D cases are: The first one is a homogeneous slab reactor from [Capilla et al., 2005], the second one is the ISSA benchmark found in various works [Issa et al., 1986], [Deo et al., 2014],[Talaat], and the third problem is a heterogeneous slab problem with 7 different regions from [Kornreich and Parsons, 2004], [Deo et al., 2014], [Vidal-Ferràndiz et al., 2018a].

The 2D cases are: first a MOX test problem found in [Capilla et al., 2008], the second one is a simple BWR cell test problem found in [Stepanek et al., 1982], [Kashi et al., 2017], the next one is the BWR rod bundle test from [Kashi et al., 2017] and the last one is the 2D C5G7 bechmark [Smith et al., 2003].

For all the cases, the diffusion coefficients are calculated by means of the following equation:

$$
D_{i, g}=\frac{1}{3 \Sigma_{t, i, g}}
$$

\subsubsection{One-dimensional cases}

Homogeneous slab reactor

This homogeneous slab is $2 \mathrm{~cm}$ in length. The one-group cross sections are defined by $\Sigma_{t}=1.0, \nu \Sigma_{f}=0.25$ and $\Sigma_{s}=0.9$. The problem was extracted from Capilla et al. [2005] where analytic solutions for $P_{1}, P_{3}$ and $P_{5}$ are presented. The boundary conditions are vacuum. The problem was also calculated with PARTISN as reference value by using 2048 cells and $S_{96}$. Since, PARTISN is not able to calculate several eigenvalues, but only the first dominant eigenvalue, the problem was also calculated with n-DOTEC algorithm explained in the chapter 3 
to obtain the rest of eigenvalues. This was achieved using a 2048 discretization and $S_{96}$.

Table 4.1 shows the calculated four largest eigenvalues. Fig. 4.23 shows the calculated flux compared with Diffusion analytic solution and discrete ordinates codes. From table 4.1 and fig.4.23, it is easy to appreciate important discrepancies between discrete ordinates method and the diffusion approximation for both, eigenvalue and flux distribution. This problem is best suited for validating the diffusion method algorithm developed, because the solution is exactly agree with analytic diffusion solution from [Capilla et al., 2005], regardless of the finite difference scheme. Moreover, table 4.1 and fig.4.23 shows that both eigenvalue and flux calculated by n-DOTEC agree perfectly with PARTISN.

Table 4.1: First 4 eigenvalues - homogeneous slab reactor. (Dif. ${ }^{C}$ is the diffusion code using cell-centered sheme). (Dif. ${ }^{E}$ is the diffusion code using edge-centered Scheme).

\begin{tabular}{lcrrcc}
\hline Eig. & $P_{1}$ Analytic Sol. & FDM Diff. ${ }^{C}$ & FDM Diff. ${ }^{E}$ & n-DOTEC $S_{96}$ & PARTISN $S_{96}$ \\
\hline 1st & 0.587489 & 0.587489 & 0.587489 & 0.662933 & 0.662933 \\
2nd & 0.149135 & 0.149135 & 0.149135 & 0.229686 & - \\
3rd & 0.058380 & 0.058380 & 0.058380 & 0.126680 & - \\
4th & 0.029602 & 0.029602 & 0.029602 & 0.085656 & - \\
\hline
\end{tabular}

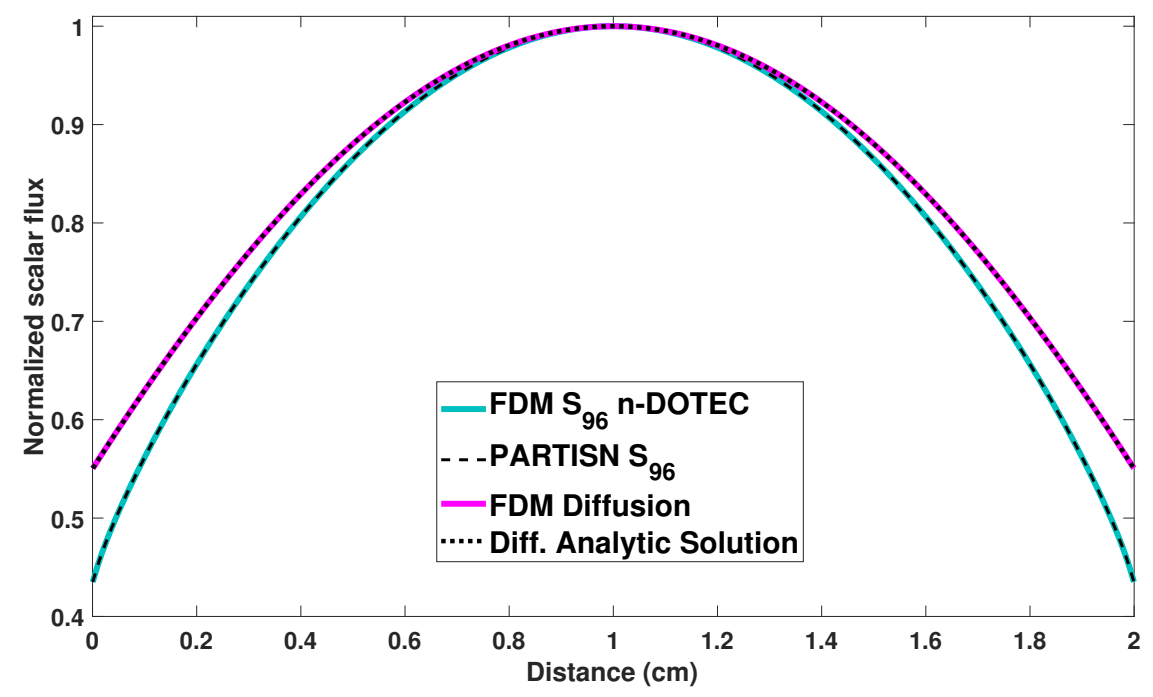

Figure 4.23: Normalized Scalar Flux for Homogeneous 1D reactor. 


\section{ISSA benchmark}

This is the same test problem presented in section 3.8.1. This is problem combines two different boundary conditions, reflective and vacuum. Fig. 4.24 shows the description of the problem.

$$
\begin{array}{ll}
\Sigma_{t 1}=1.0 & \Sigma_{t 2}=0.8 \\
\Sigma_{s 1}=0.5 & \Sigma_{s 2}=0.4 \\
v \Sigma_{f 1}=1.0 & v \Sigma_{f 2}=0.0
\end{array}
$$

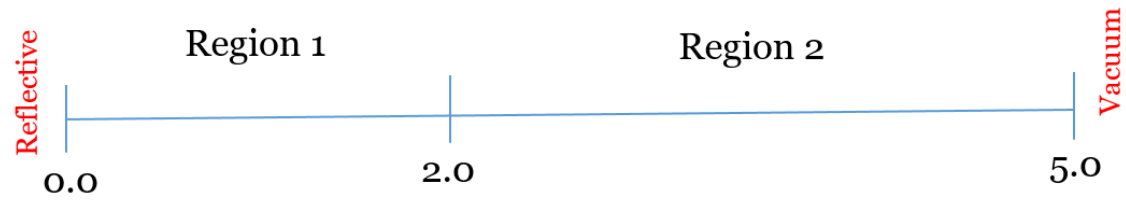

Figure 4.24: Geometry and cross sections for ISSA Benchmark.

For this section, the problem was calculated by using a 2000 cells. The discrete ordinates order of the PARTISN code and FDM n-DOTEC was $S_{96}$. Table 4.2 presents the eigenvalues comparison for the four first modes. The results show a huge difference between $S_{96}$ and diffusion, about $2000 \mathrm{pcm}$ for the first mode and this difference is higher for the $2^{\text {nd }}, 3^{\text {rd }}$ and $4^{\text {th }}$. Figs.4.25-4.28 show the normalized scalar flux corresponding to each calculated mode. Contrary to the 1D homogeneous problem, ISSA case shows good flux agreement close to the boundary, but the results are not good at the interface separating the two regions. Moreover, this difference seem to be higher in the next eigenfunctions.

Table 4.2: ISSA problem eigenvalues.

\begin{tabular}{lcccc}
\hline modes & PARTISN $S_{96}$ & FDM $S_{96}$ n-DOTEC & FDM Diff. ${ }^{C}$ & FDM Diff. $^{E}$ \\
\hline 1st & 1.678794 & 1.678799 & 1.645685 & 1.645684 \\
2nd & - & 0.801363 & 0.604050 & 0.604049 \\
3rd & - & 0.465249 & 0.239767 & 0.239766 \\
4th & - & 0.321525 & 0.120525 & 0.120524 \\
\hline
\end{tabular}




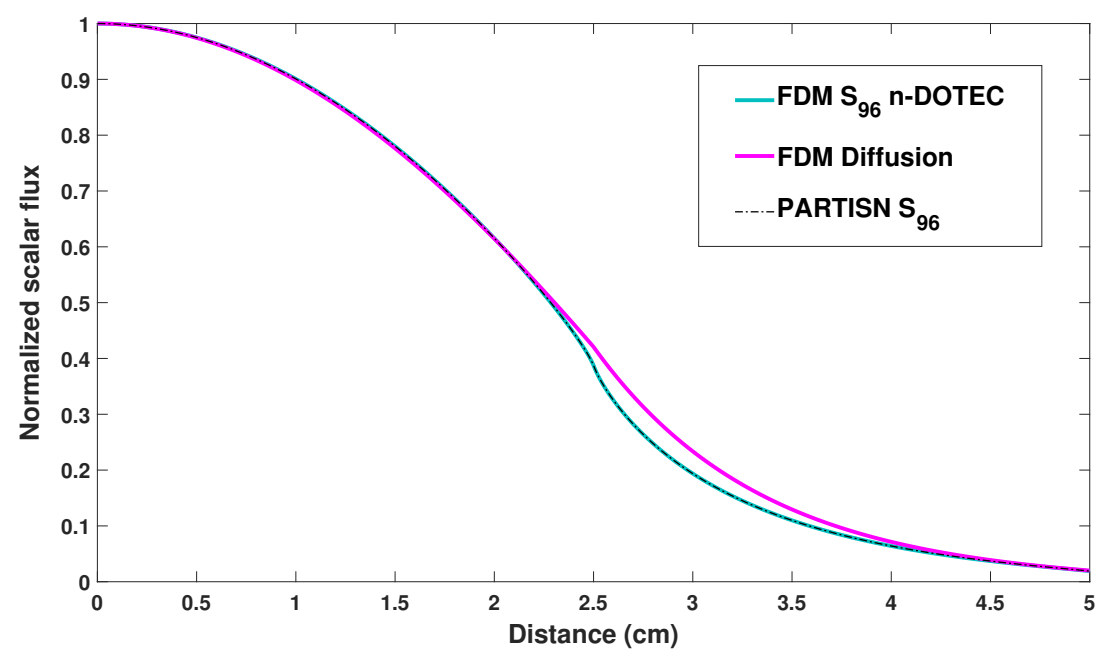

Figure 4.25: ISSA: normalized scalar flux for the first mode.

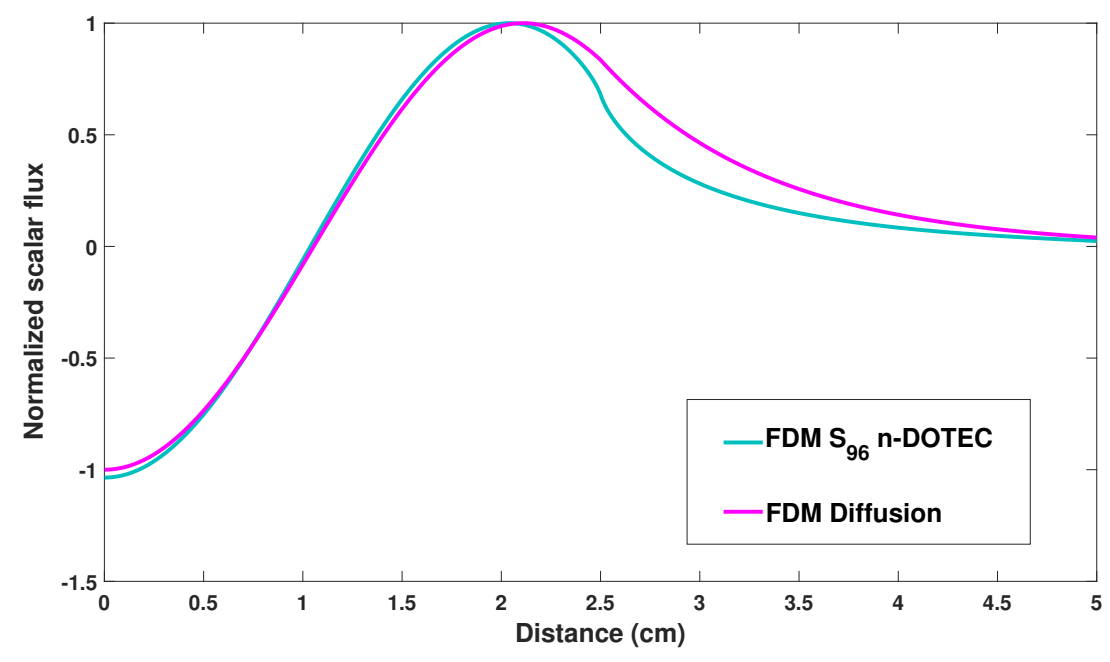

Figure 4.26: ISSA: normalized scalar flux for the second mode. 


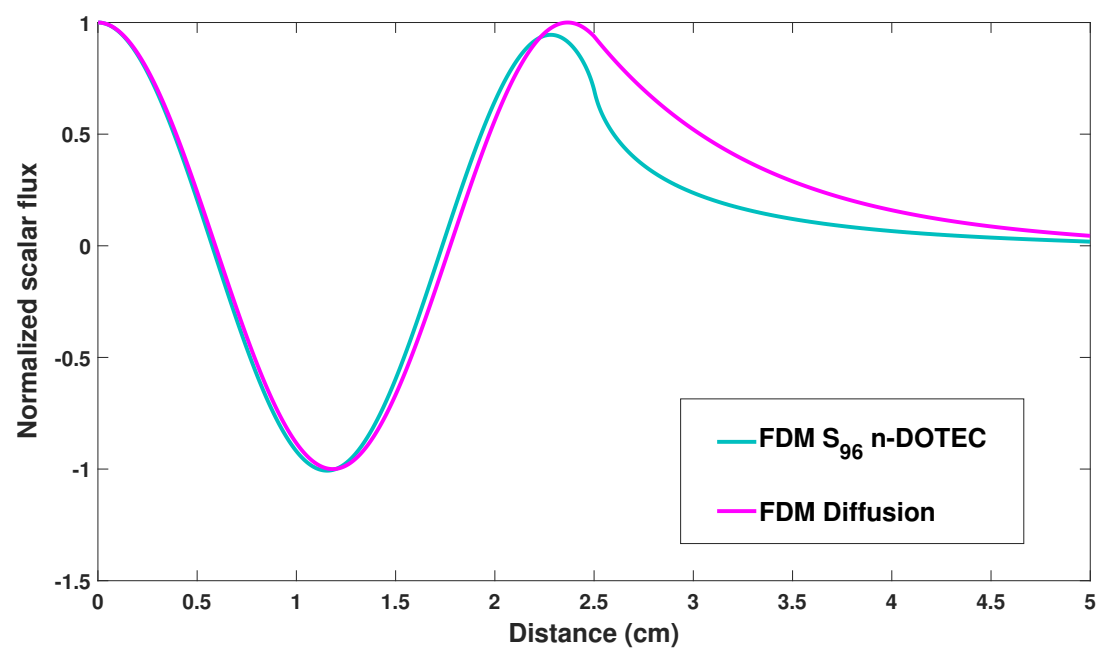

Figure 4.27: ISSAe: normalized scalar flux for the third mode.

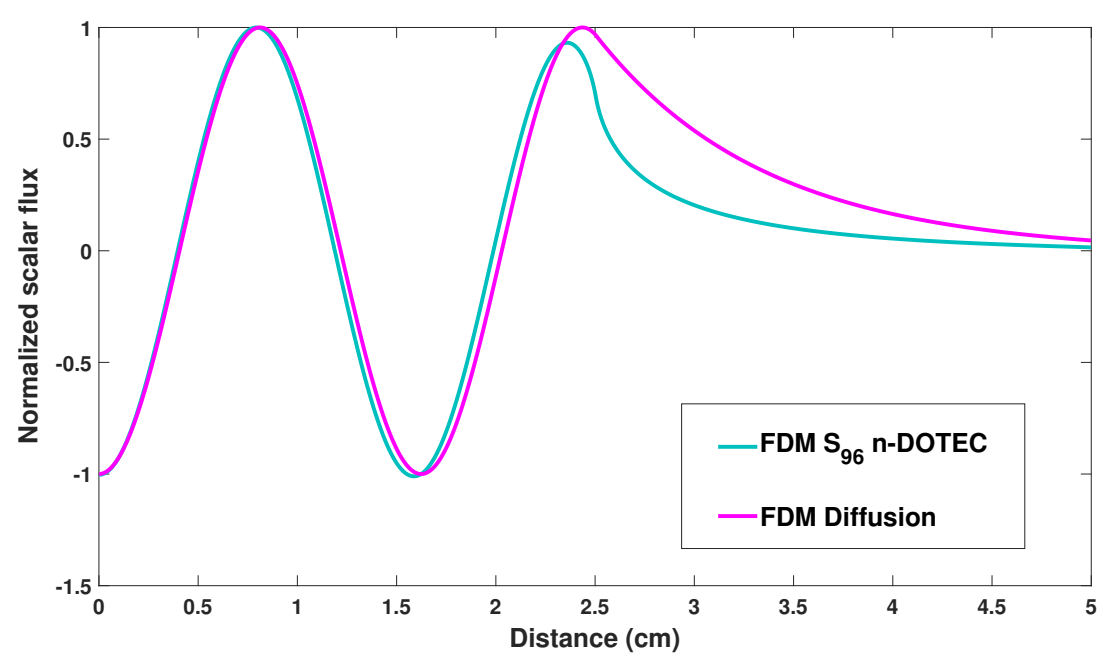

Figure 4.28: ISSA: normalized scalar flux for the fourth mode. 
Heterogeneous slab problem

The cross sections of this problem were extracted from Capilla et al. [2005] and are shown in table 4.3.

Table 4.3: Cross-Sections for Heterogeneous slab problem.

\begin{tabular}{lccc}
\hline & $\nu \Sigma_{f}\left(\mathrm{~cm}^{-1}\right)$ & $\Sigma_{s}\left(\mathrm{~cm}^{-1}\right)$ & $\Sigma_{t}\left(\mathrm{~cm}^{-1}\right)$ \\
\hline Fuel (U-235) & 0.178 & 0.334 & 0.416667 \\
Reflector (Be) & 0.0 & 0.334 & 0.37037 \\
Absorber (Be $w / c=0.1)$ & 0.0 & 0.037 & 0.37037 \\
\hline
\end{tabular}

Base case

\begin{tabular}{|l|l|l|l|l|l|l|}
$R$ & Fuel & $R$ & Fuel & $R$ & Fuel & $R$ \\
\hline
\end{tabular}

Abs. in Pos. 5

\begin{tabular}{l|l|l|l|l|l|l}
$R$ & Fuel & $R$ & Fuel & Abs. & Fuel & $R$ \\
\hline
\end{tabular}

Abs. in Pos. 6

\begin{tabular}{l|l|l|l|l|l}
$R$ & Fuel & $R$ & Fuel & $R$ & Abs. \\
\hline
\end{tabular}

Figure 4.29: Cases for Heterogeneous slab problem.

Three versions of the problem are defined depending on the position of the absorber material according to fig.4.29. Table 4.4 shows the modes comparison between the diffusion method and the discrete ordinates. It is important to highlight that the considerable difference between these methods for the 4 dominant eigenvalues, which is greater than $4000 \mathrm{pcm}$. Furthermore, there are also discrepancies in the scalar flux as shown in figs. 4.30, 4.34 and 4.35. These cases bring out the discrepancies beyond the zones close to the boundary conditions, since there are several zones where the change of material means important differences between the values calculated by diffusion equation and discrete ordinates method.

In addition, fig.4.30-4.33 show the results corresponding to the scalar flux of the fourth mode. Although the order of the largest eigenvalues and eigenfunctions are not affected by changing the angular approximation method, it should be noted that appear important differences between both methodologies. 
Table 4.4: Heterogeneous slab problem eigenvalues.

\begin{tabular}{lcccc}
\hline Case & modes & PARTISN $S_{96}$ & FDM $S_{96}$ n-DOTEC & FDM Diff. ${ }^{C, E}$ \\
\hline No absorber & 1st & 1.162413 & 1.162228 & 1.113872 \\
& 2nd & - & 0.752258 & 0.658651 \\
& 3rd & - & 0.547565 & 0.423945 \\
& 4th & - & 0.210955 & 0.109256 \\
\hline Abs. in Pos. 5 & 1st & 0.933891 & 0.934122 & 0.846061 \\
& 2nd & - & 0.650383 & 0.533335 \\
& 3rd & - & 0.525017 & 0.401738 \\
& 4th & - & 0.202416 & 0.101340 \\
\hline Abs. in Pos. 6 & 1st & 1.013832 & 1.013802 & 0.943324 \\
& 2nd & - & 0.598874 & 0.480401 \\
& 3rd & - & 0.207386 & 0.105354 \\
& 4th & - & 0.187820 & 0.084083 \\
\hline
\end{tabular}

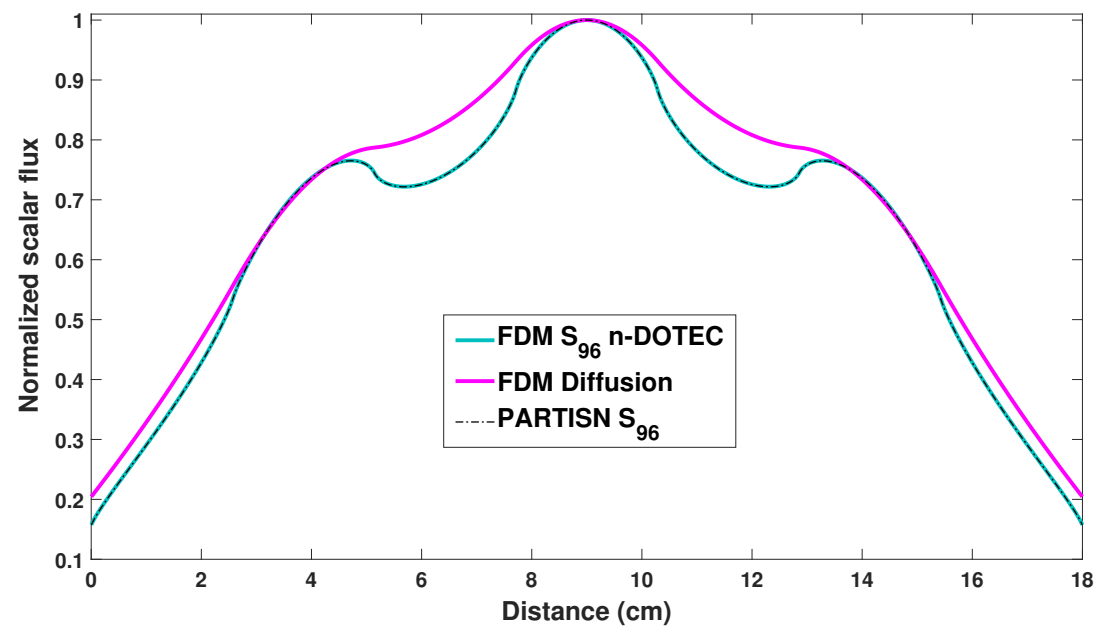

Figure 4.30: Basecase: normalized scalar flux for the first mode. 


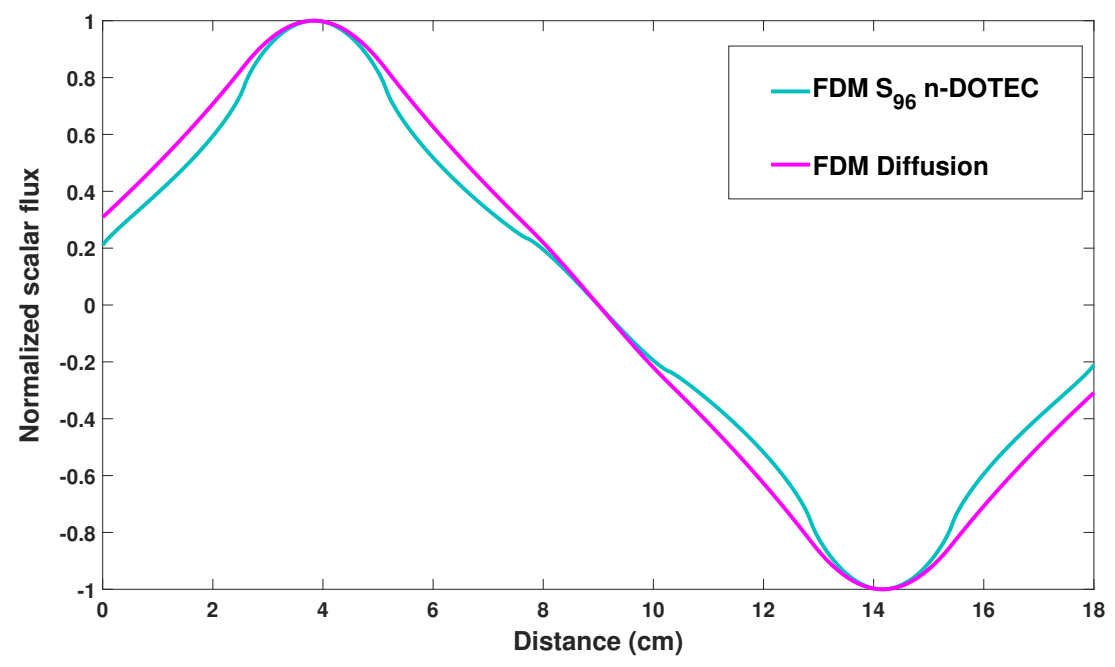

Figure 4.31: Basecase: normalized scalar flux for the second mode.

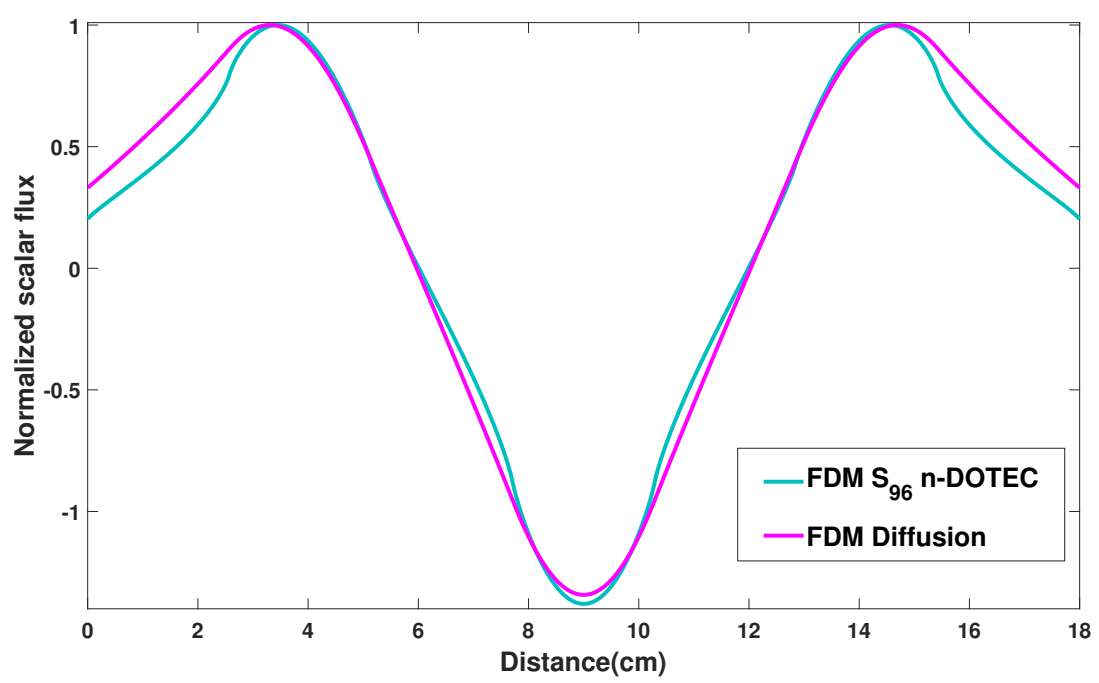

Figure 4.32: Basecase: normalized scalar flux for the third mode. 


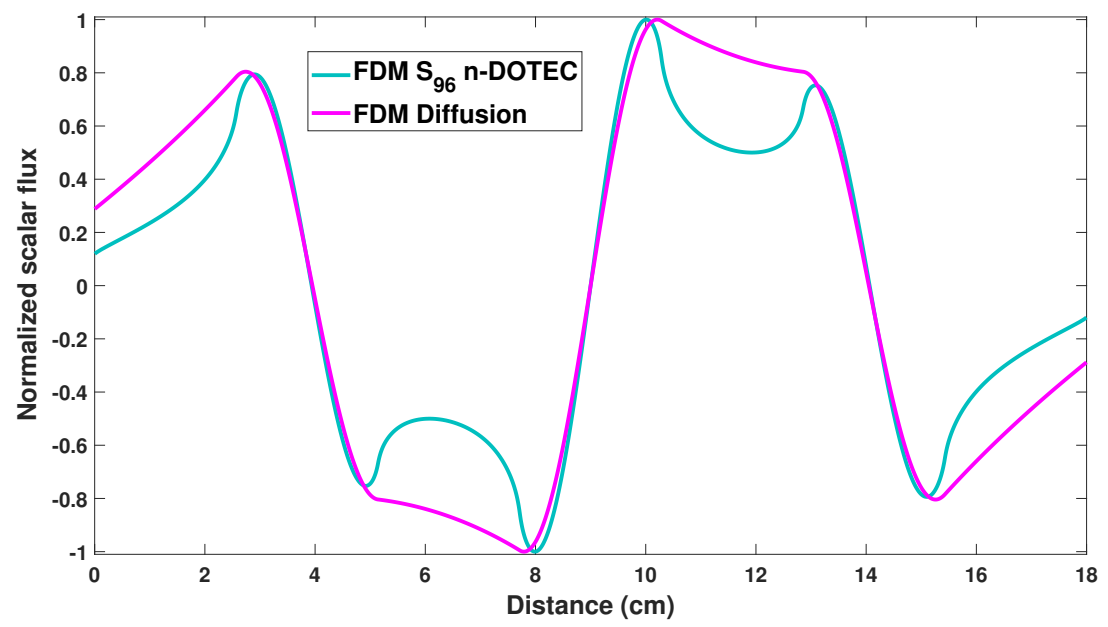

Figure 4.33: Basecase: normalized scalar flux for the fourth mode.

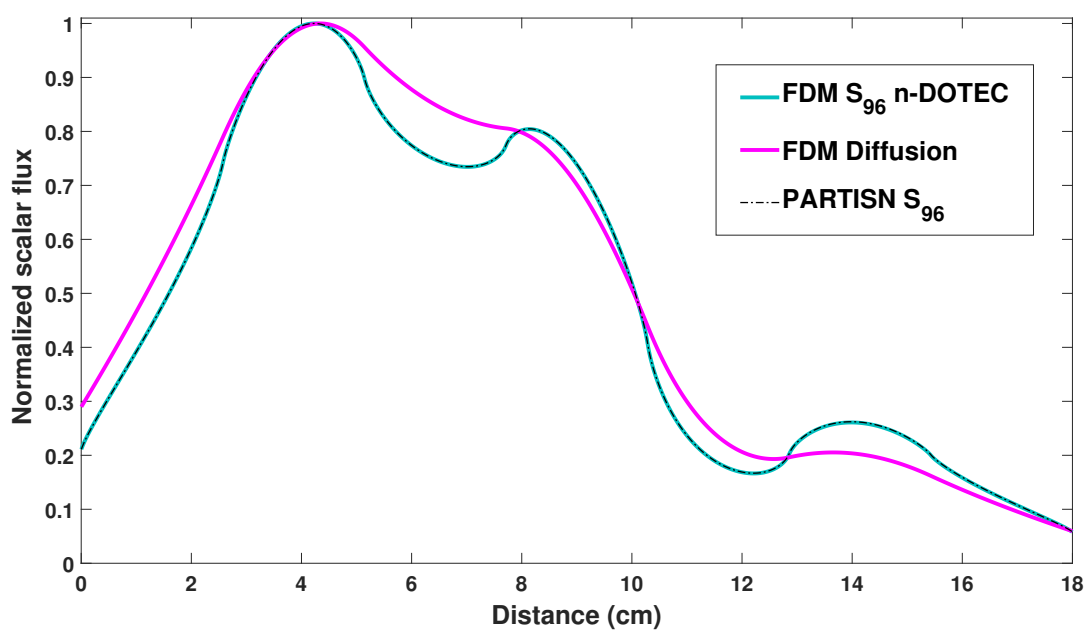

Figure 4.34: Absorber Position 5: normalized scalar flux for the first mode. 


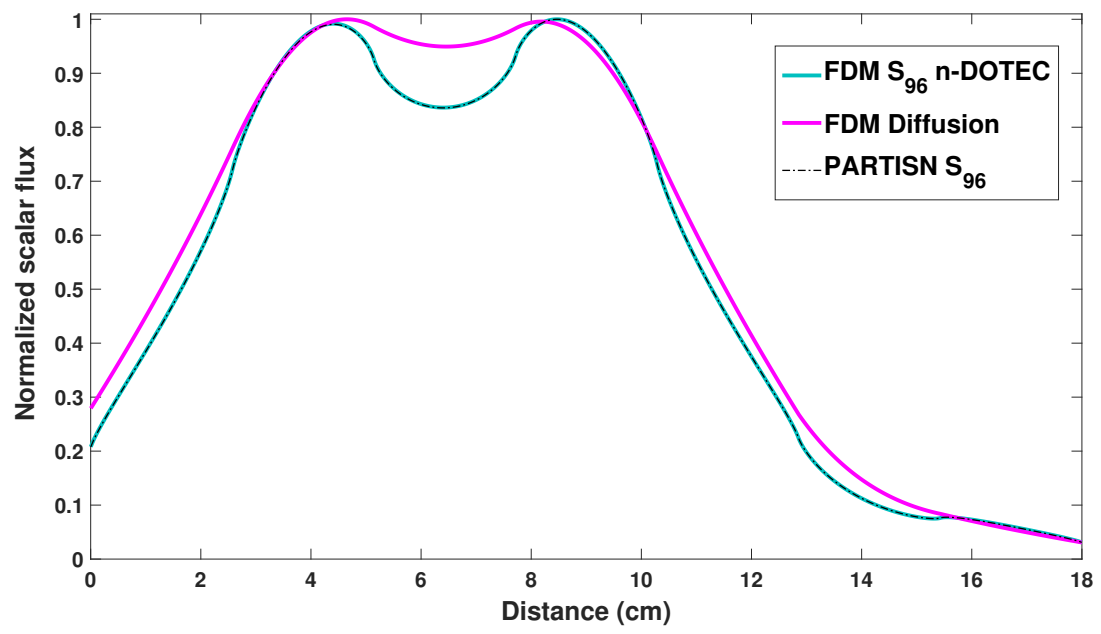

Figure 4.35: Absorber Position 6: normalized scalar flux for the first mode. 


\subsubsection{Two-dimensional cases}

\section{MOX test problem}

This problem was defined in section 3.8.2. However, in this section, the order of the discrete ordinates was $S_{8}$ to make a better comparison. The discretization for both methods, discrete ordinates and diffusion is 10 cells in $x$ and $y$ axis for each assembly. In figs. 4.36, 4.37 and 4.38, the scalar flux distribution is displayed for the first, second, third and fourth modes.

Table 4.5 shows the results for the 4 largest eigenvalues with the reference SHNC, $D i f^{C}$ which is cell-centered scheme diffusion code, $D i f^{E}$ which is edge-centered scheme diffusion code and n-DOTEC $S_{8}$. It can be appreciated from table 4.5 that the cell-centered diffusion scheme shows higher precision compared with the edge-centered. However, considering the eigenfunctions shown at figs. 4.36-4.38, there are no important differences between diffusion (cell centered and edge centered are represented by the same line) and the discrete ordinates method in this case. This results from the fact that MOX problem is a higher diffusive problem as can be seen in the cross sections of the table 3.5 where the scattering terms have a significant contribution contrarily to the absorption.

Although, one could think that the eigenfunctions for the sub-critical modes are different,it must be taken into account that the different rotation of the modes is correct and it is because the symmetry of the problem. Furthermore, taking a look to the $4^{\text {th }}$ mode, one can be appreciate that it is a linear combination of the $2^{\text {nd }}$ and $3^{\text {rd }}$ modes. So this solutions are valid and mathematically consistent.

So, it is rotated because it has not converged correctly. It is not that the solution is wrong, but it is that the eigenvector that SLEPc has given is a linear combination of the second and third, because they share the same eigenvalue. Therefore, any linear combination of both eigenvectors is also an eigenvector of the same eigenvalue. Mathematically, it would be next explanation. Suppose there are two eigenvectors $x_{1}$ and $x_{2}$ for matrix $A$ with the same eigenvalue $I$. Then it is satisfied: $A x_{1}=I x_{1}$ and $A x_{2}=I x_{2}$. Suppose a vector $x_{3}=b_{1} x_{1}+b_{2} x_{2}$, where $b_{1}$ and $b_{2}$ are any real number. If it is calculated the product of $A$ and $x_{3}$, one can obtain $A x_{3}=A\left(b_{1}+b_{2} x_{2}\right)=b_{1} A x_{1}+b_{2} A x_{2}=b_{1} I x_{1}+b_{2} I x_{2}=I\left(b_{1} x_{1}+b_{2} x_{2}\right)=$ $I x_{3}$. That is, $x_{3}$ is an eigenvvector of $A$ for the eigenvalue $I$. 


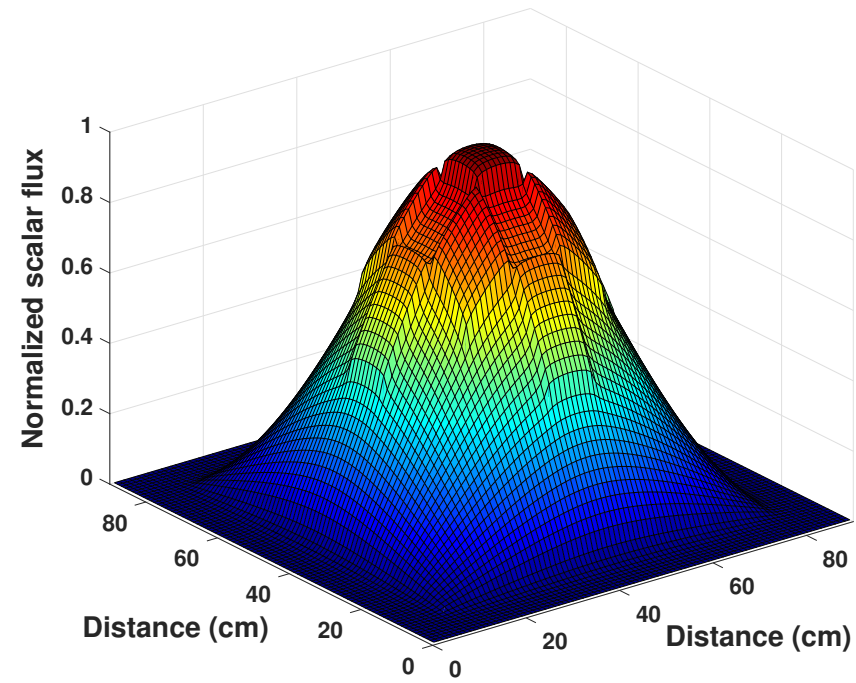

(a) Diffusion.

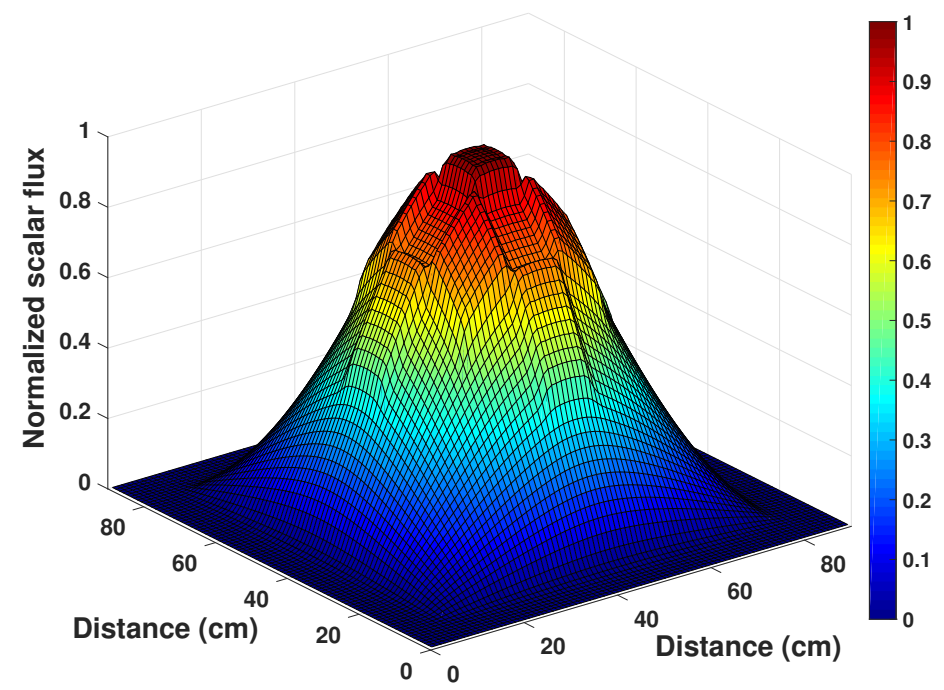

(b) Discrete ordinates $S_{8}$.

Figure 4.36: MOX: First energy group scalar flux distribution for 1st mode. 
Table 4.5: Dominant eigenvalues for the MOX problem. *SHNC=Spherical Harmonics Nodal Collocation.

\begin{tabular}{ccccc}
\hline Eigenvalue & SHNC* $^{*}$ & FDM Dif. & & \\
\hline$k_{\text {eff }}$ & 0.9925 & FDM Dif. & E* & FDM $S_{8}$ \\
2nd eigen. & 0.9665 & 0.9666665 & 0.993133 & 0.992608 \\
3rd eigen. & 0.9665 & 0.966665 & 0.966868 & 0.966544 \\
4th eigen. & 0.9399 & 0.939807 & 0.939626 & 0.966544 \\
\hline
\end{tabular}
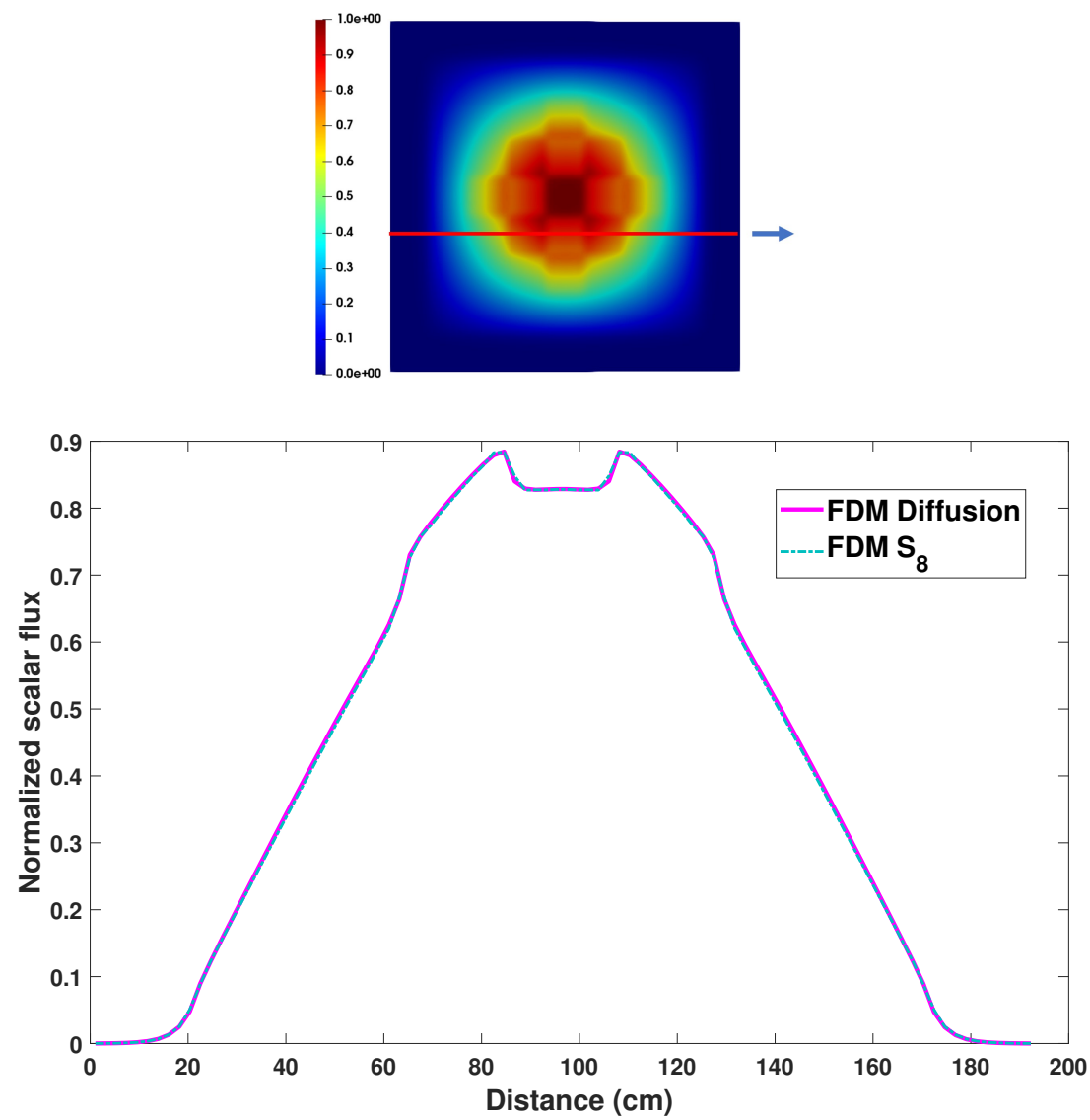

Figure 4.37: MOX: Profile comparison for the first energy group scalar flux distribution of the 1st eigenvalue. 

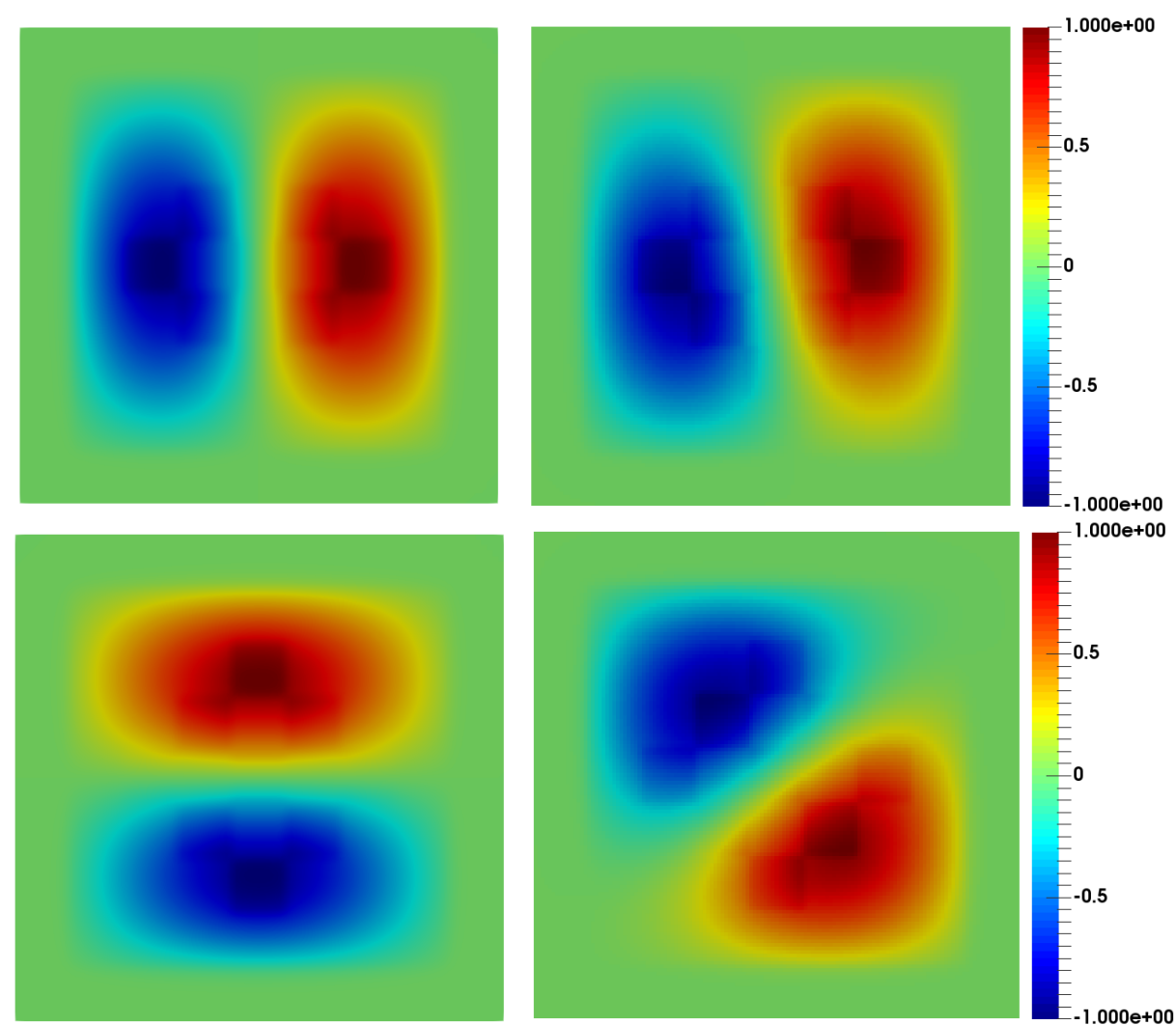

$-1.000 e+00$
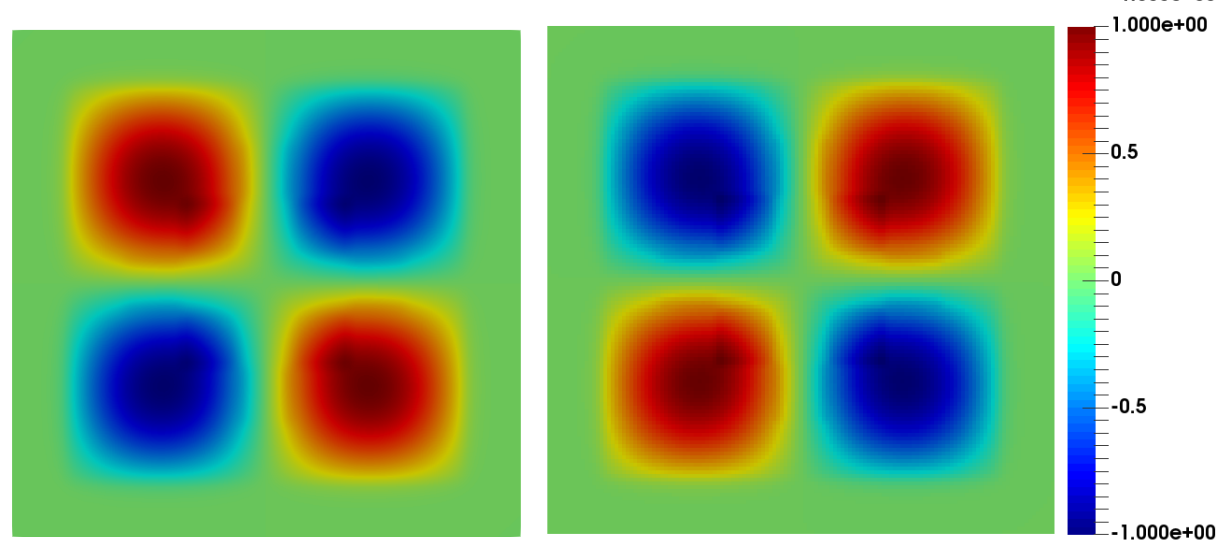

Figure 4.38: MOX: Normalized Scalar flux distribution for $2^{\text {nd }}, 3^{\text {rd }}$ and $4^{\text {th }}$ modes, FDM diffusion on the left, FDM $S_{8}$ on the right. 
BWR cell test problem

As mentioned before in the neutron transport chapter, section 3.8.2, the consideration of the upscattering in this case is one of the reasons why it was selected for this work. The reference multiplication factor calculated with DANTSYS code (that uses the discrete ordinates method) is 1.212945. FDM $S_{8}$ and diffusion results were calculated by using a discretization of 30x30 meshes. Table 4.6 shows the results for the multiplication factor $k_{e f f}$. A flux comparison between $S_{8}$ and diffusion can be seen in fig.4.40 and 4.41. Fig.4.42 shows the first group flux neutron distribution for the four dominant eigenvalues.

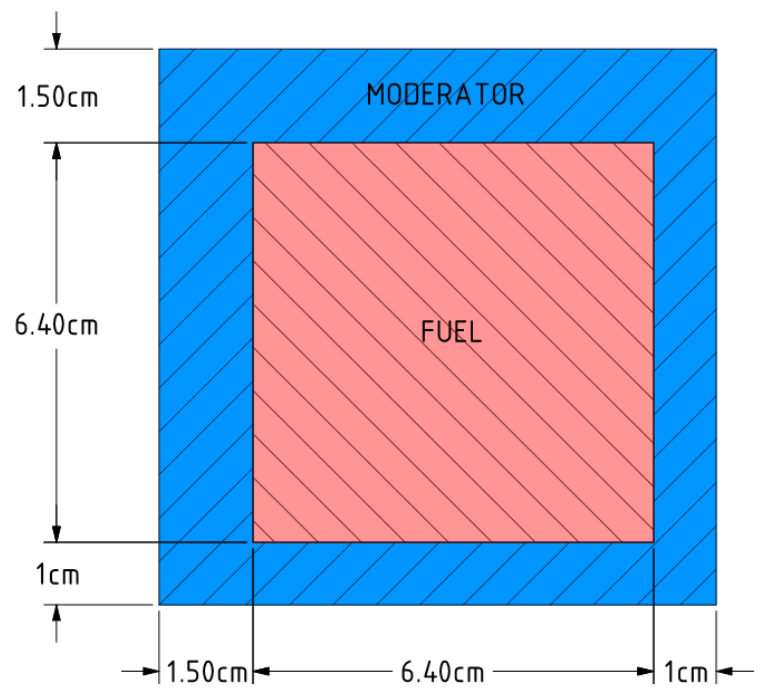

Figure 4.39: BWR cell problem geometry.

Table 4.6: BWR cell benchmark problem multiplication factor results.

\begin{tabular}{cccc} 
& Order & $k_{\text {eff }}$ & $\mathrm{pcm}\left(\Delta K_{\text {eff }}\right)$ \\
\hline DANTSYS & $S_{8}$ & 1.212945 & - \\
FDM $S_{N}$ & $S_{8}$ & 1.212944 & 0 \\
FDM Dif.C & - & 1.220100 & 589 \\
FDM Dif. ${ }^{E}$ & - & 1.220886 & 654 \\
\hline
\end{tabular}



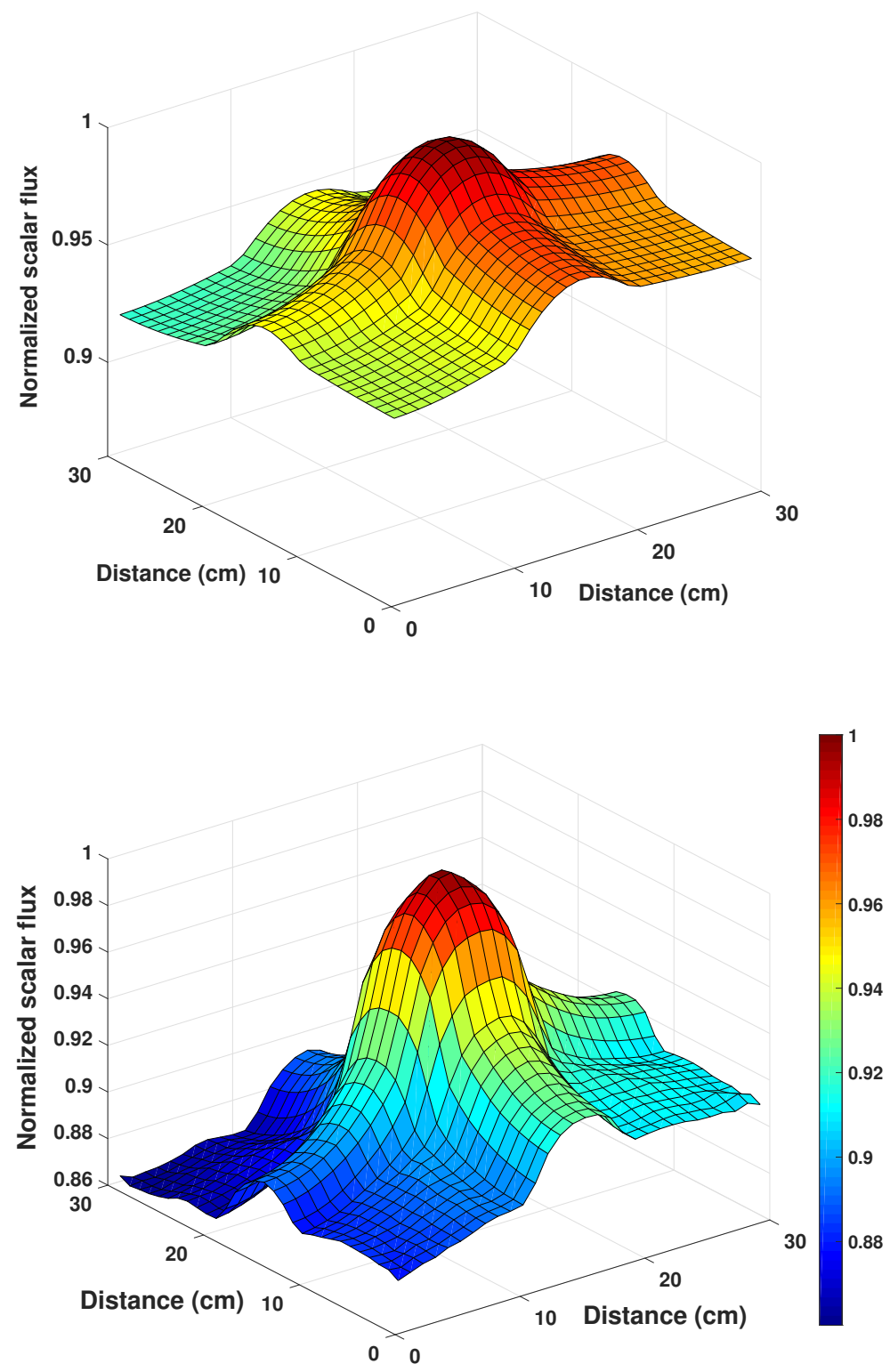

Figure 4.40: BWR cell test: scalar flux distribution for the $1^{\text {st }}$ mode, $1^{\text {st }}$ energy group. Diffusion and Discrete Ordinates, respectively. 

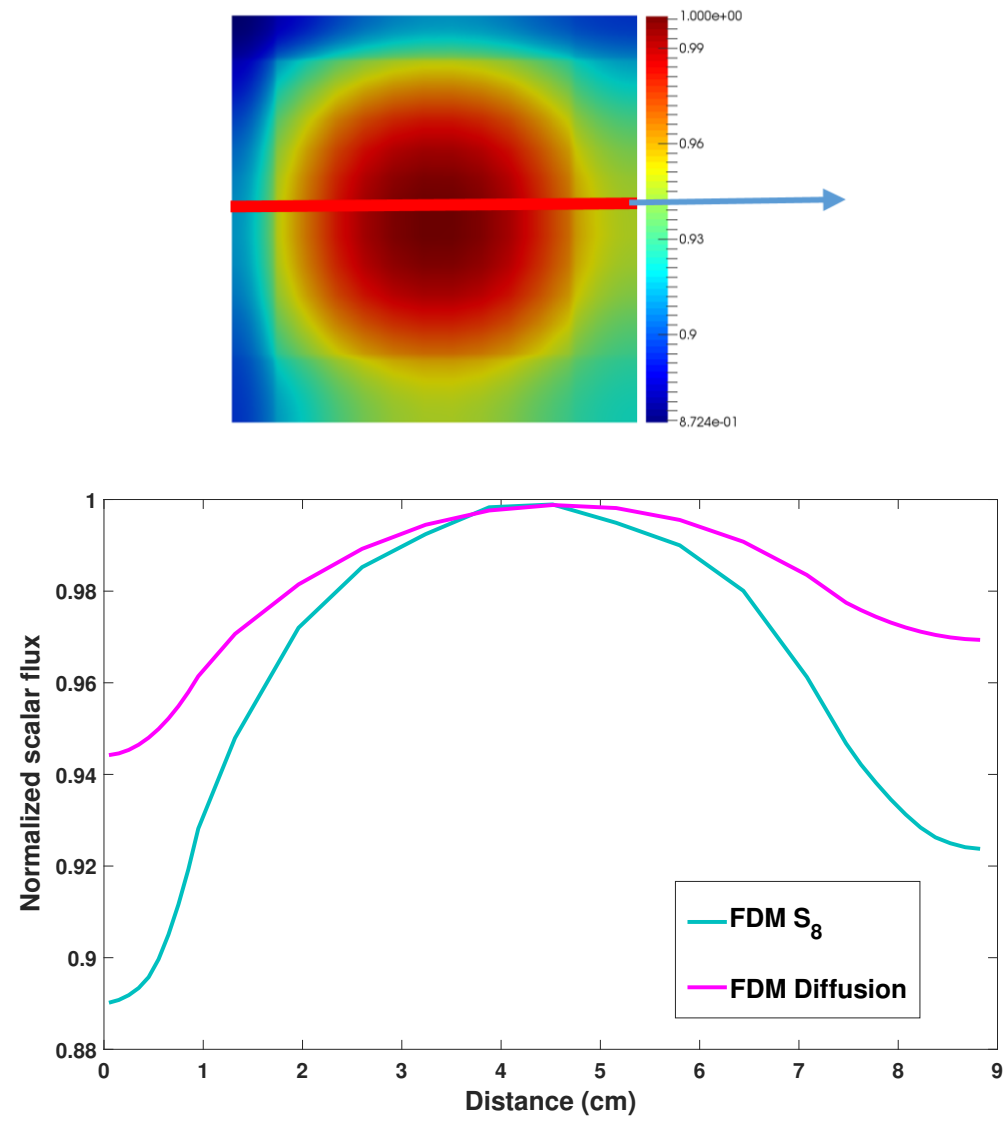

Figure 4.41: BWR cell test: scalar flux distribution for the $1^{\text {st }}$ mode, $1^{\text {st }}$ energy group. 

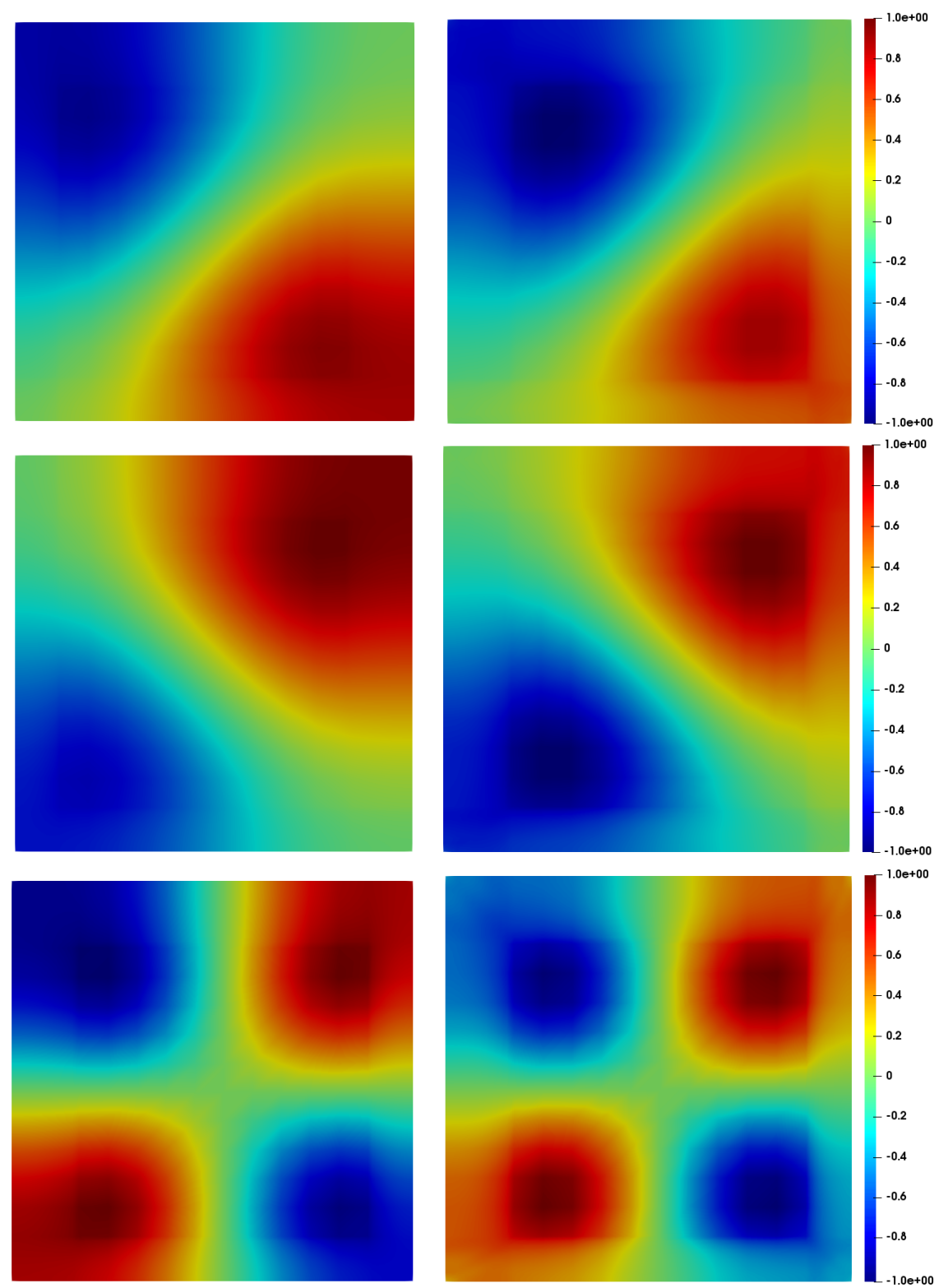

Figure 4.42: Normalized scalar flux of the first four modes for the BWR cell test problem. FDM Diffusion on the left, FDM $S_{8}$ on the right. 
$B W R$ rod bundle test problem

This case was described at section 3.8.2. However, this section uses a discretization of $10 \times 10$ cells, as shown in fig.4.43. The reference value was calculated with DANTSYS code using order $S_{16}$ and the same mesh with 14400 cells. Several results were calculated, as table 4.7 shows. The problem was solved with FDM $S_{6}, S_{8}$, cell centered scheme FDM Diff (FDM Diff. ${ }^{C}$ ) and edge centered scheme FDM Diff (FDM Diff. ${ }^{E}$ ). Important discrepancies are found between discrete ordinates and diffusion. Although the error of the first eigenvalue is less than 200 $\mathrm{pcm}$, the errors of the rest of eigenvalues are greater than $9000 \mathrm{pcm}$. Moreover, figs.4.44 and 4.45 show great differences in the flux.

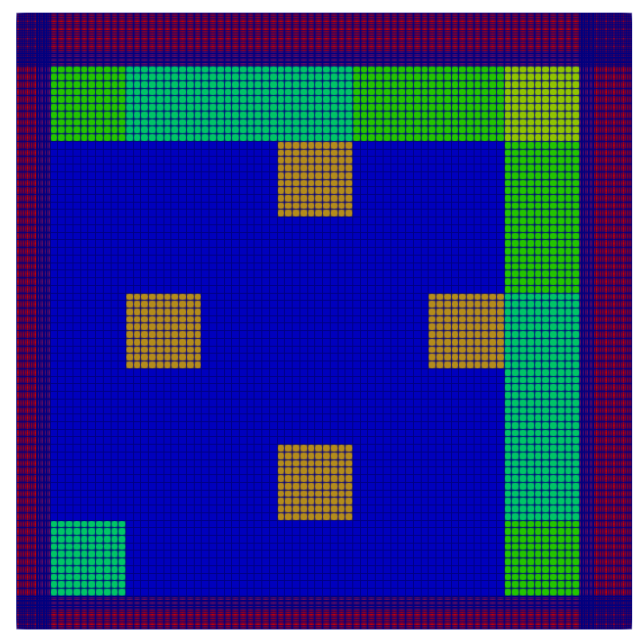

Figure 4.43: BWR rod bundle mesh Mesh.

Table 4.7: Eigenvalues comparison. ${ }^{1}$ SHNC=Spherical Harmonics Nodal Collocation Capilla et al. [2018].

\begin{tabular}{cccccc}
\hline eigenvalue $->$ & 1st & 2nd & 3rd & 4th & pcm $\left(\Delta K_{\text {eff }}\right)$ 1st \\
\hline DANTSYS $S_{16}$ & 1.090330 & - & - & - & - \\
FDM $S_{6}$ & 1.090922 & 0.241339 & 0.236108 & 0.117371 & 54 \\
FDM $S_{8}$ & 1.090651 & 0.241211 & 0.235994 & 0.117099 & 29 \\
FDM Diff. ${ }^{C}$ & 1.088671 & 0.218218 & 0.213061 & 0.093681 & 152 \\
FDM Diff. & 1.088504 & 0.218241 & 0.213083 & 0.093703 & 167 \\
\hline
\end{tabular}



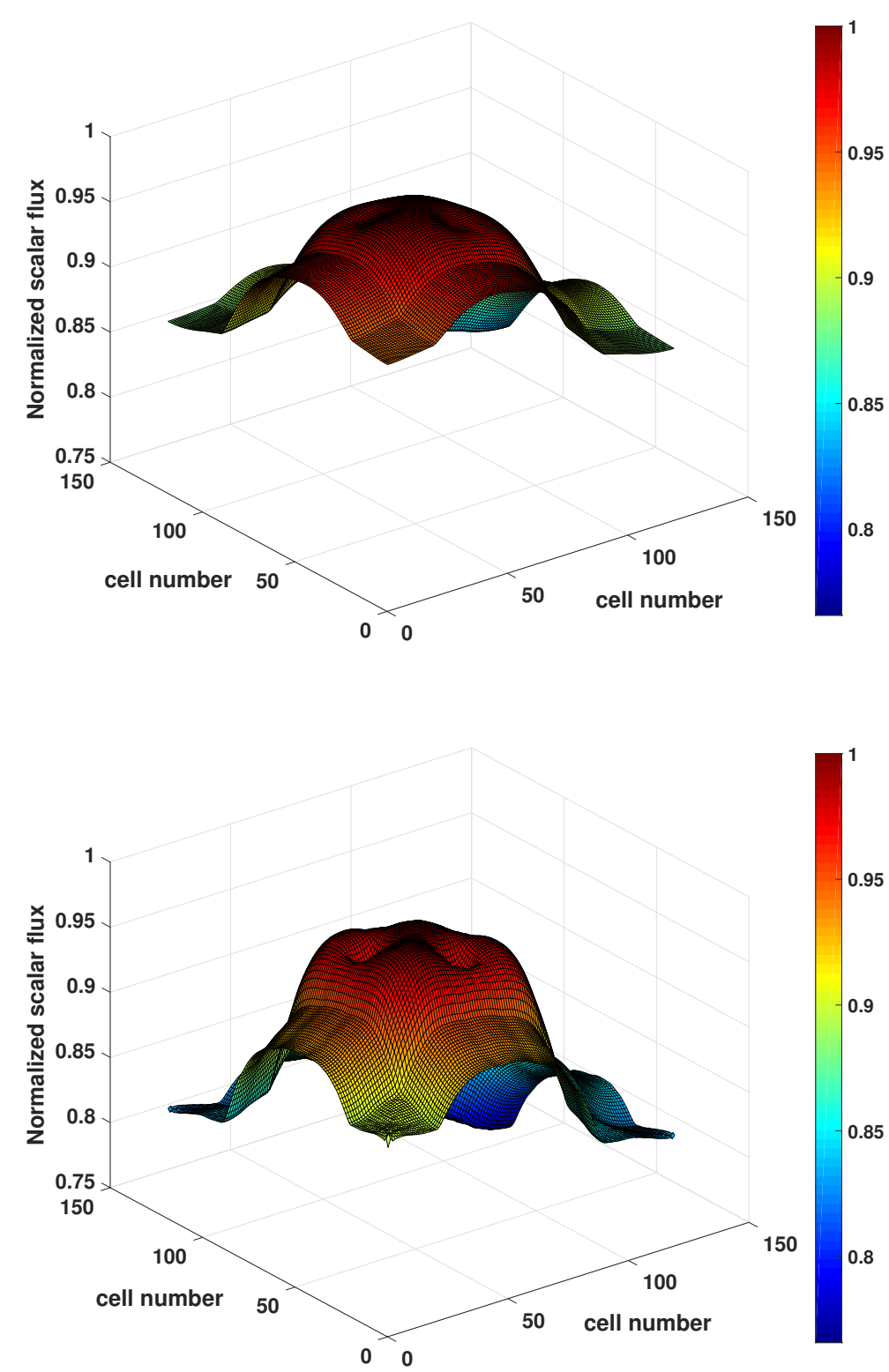

Figure 4.44: BWR rod bundle test: scalar flux distribution for the 1st mode. Diffusion and Discrete Ordinates, respectively. 

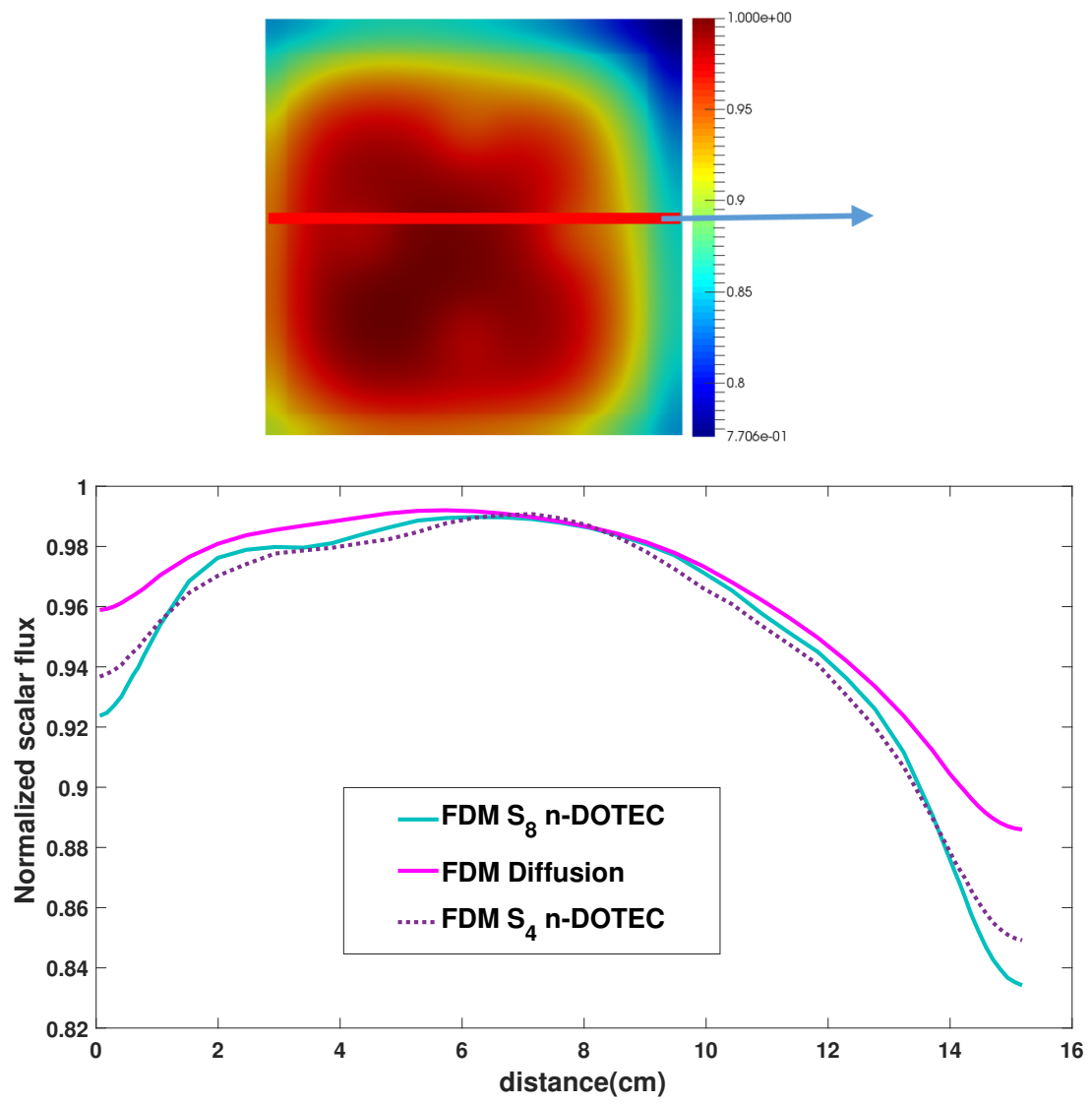

Figure 4.45: BWR rod bundle test: scalar flux distribution for the 1st mode. 

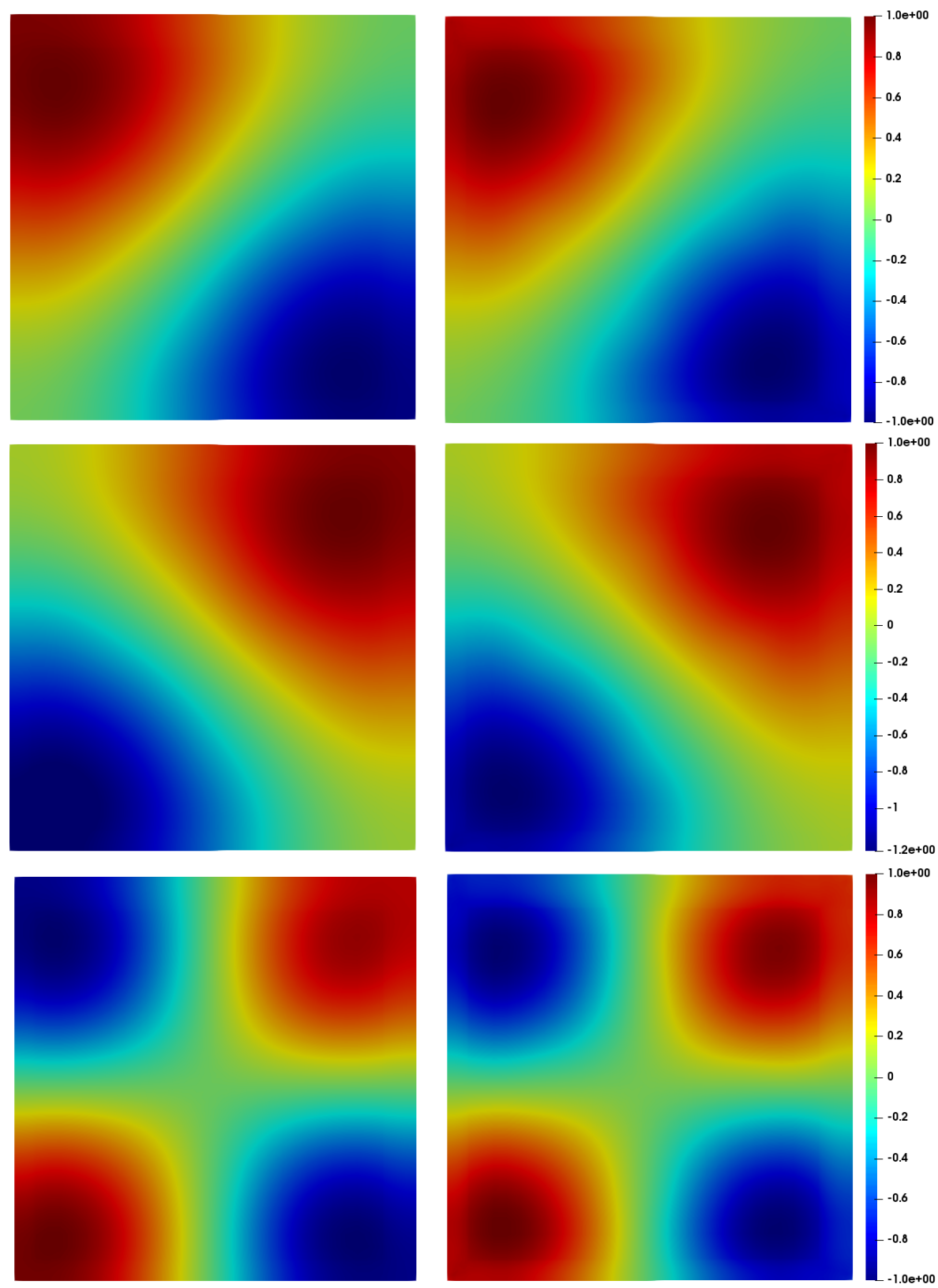

Figure 4.46: Notmalized scalar flux distribution of the first four modes for the BWR rod bundle test problem. Diffusion on the left and discrete ordinates on the right. 
Two-dimensional C5G7 test problem

This benchmark corresponds to a quarter symmetry core problem and it was described in section 3.8.2. Since, in this work, algorithms are limited to the use of Cartesian geometry, the cylindrical pin is approximated by a square with the same area as the corresponding cylinder. Four different meshes are considered for the core, which can be seen in fig.4.47. The 7 energy groups cross-sections can be found in the benchmark Smith et al. [2003] for the seven corresponding materials. The reactor is comosed of three MOX pin fuels with different enrichments, $\mathrm{UO}_{2}$ fuels, guide tubes, fission chambers and moderator. Tables 4.8-4.11 summarize the results comparison obtained by FDM Diffusion with FDM $S_{N}$ and those obtained by MCNP, which are provided in the reference solution.

A sensitive analysis of the mesh was performed, which is shown in tables 4.9 and 4.10. From these tables, two conclusions are drawn. First, for the cell-centered scheme, the finer the mesh, the lower the eigenvalue error. Second, for the edgecentered scheme, the finer the mesh, the greater the error. However, the variation of the error, in both cases, is less than $100 \mathrm{pcm}$.

With respect to the power errors, the cell-centered results presents higher maximum percentage error and RMS when the mesh is becoming finer, but the values of AVG and MRE decrease when the mesh is finer.

Contrarily, table 4.10 shows greater eigenvalue errors if the mesh is finer, but lower values of the rest of calculated errors.

In addition, another conclusion can be drawn from table 4.11, just with lower discrete ordinates $S_{4}$ the results are much better than diffusion even when it uses a finer mesh.

Table 4.8: Eigenvalues comparison. ${ }^{1} S H N C=$ Spherical Harmonics Nodal Collocation Capilla et al. [2018].

\begin{tabular}{ccccccc}
\hline eigenvalue $->$ & 1st & 2nd & 3rd & 4th & 5th & 6th \\
\hline Ref. MCNP & 1.186550 & - & - & - & - & - \\
FDM S2 & 1.188877 & 0.912220 & 0.868536 & 0.730518 & 0.571266 & 0.570752 \\
FDM S4 & 1.187619 & 0.918477 & 0.873095 & 0.727383 & 0.592365 & 0.591601 \\
${ }^{1}$ SHNC P1 & 1.183847 & 0.904490 & 0.859548 & 0.703131 & 0.562243 & 0.561512 \\
1 1 FDM Dif. & 1.182095 & 0.901555 & 0.857969 & 0.700217 & 0.559826 & 0.559129 \\
\hline
\end{tabular}



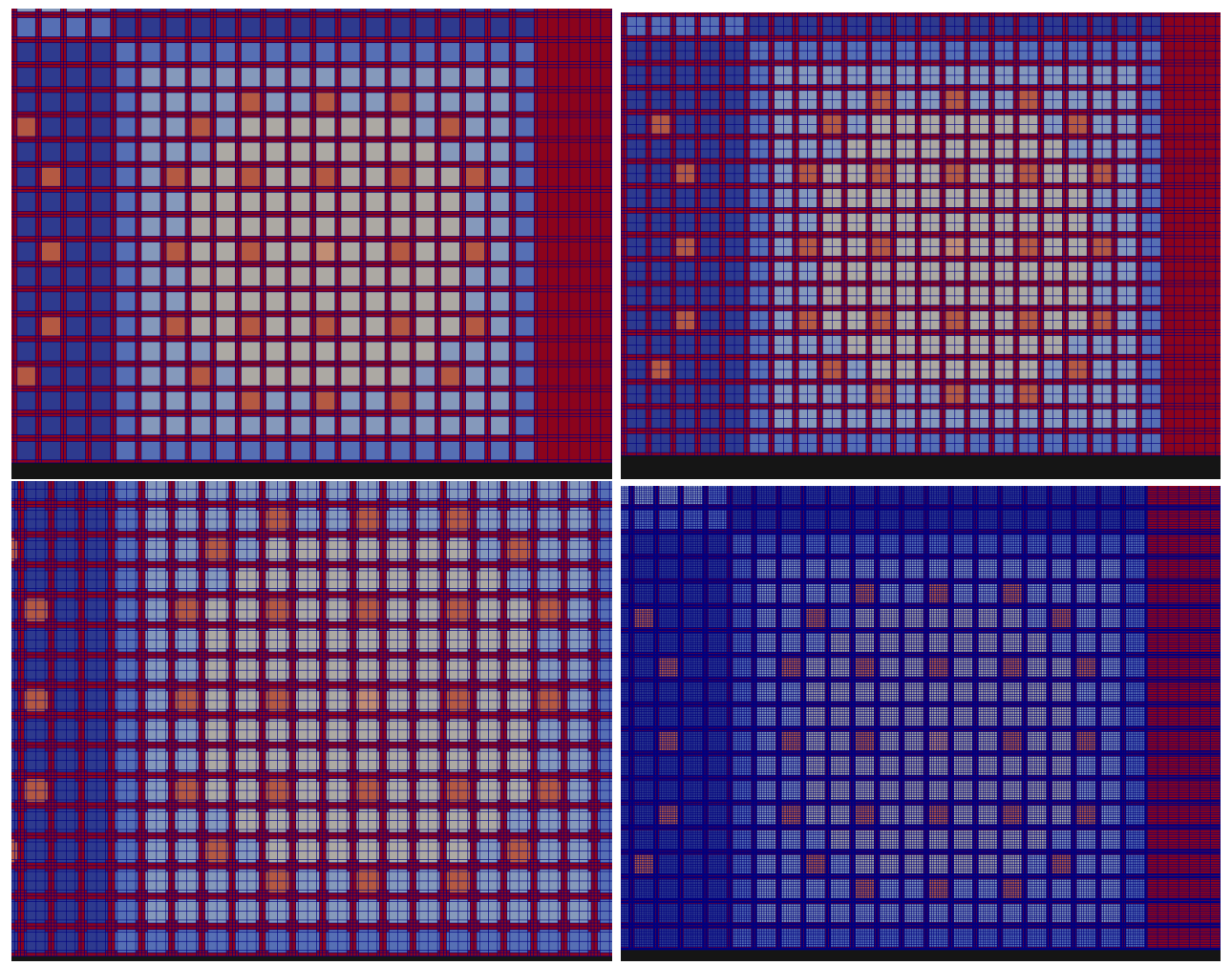

Figure 4.47: Detail of $1 \times 1,4 \times 4,6 \times 6$ and $8 \times 8$ meshes.

Table 4.9: $k_{e f f}$ and power comparison by using cell-centered Scheme.

\begin{tabular}{ccccccccc}
\hline & Discret. & $N^{o}$ of elem. & $k_{\text {eff }}$ & pcm & Max.Perc.Error & AVG & RMS & MRE \\
\hline MCNP & - & - & 1.186550 & - & - & - & - & - \\
Dif. & $1 \times 1$ & 17424 & 1.182095 & 375 & 6.480 & 2.326 & 2.669 & 2.105 \\
Dif. & $4 \times 4$ & 191844 & 1.183066 & 294 & 7.385 & 2.223 & 2.807 & 2.015 \\
Dif. & $6 \times 6$ & 412164 & 1.183032 & 296 & 7.748 & 2.258 & 2.867 & 2.050 \\
Dif. & $8 \times 8$ & 715716 & 1.183935 & 220 & 8.280 & 2.298 & 2.957 & 2.080 \\
\hline
\end{tabular}


Table 4.10: $K_{\text {eff }}$ and power comparison by using edge-centered scheme.

\begin{tabular}{ccccccccc}
\hline & Discret. & $N^{o}$ of elem. & $k_{\text {eff }}$ & pcm & Max.Perc.Error & AVG & RMS & MRE \\
\hline MCNP & - & - & 1.186550 & - & - & - & - & - \\
Dif. & $1 \times 1$ & 17689 & 1.184963 & 134 & 11.368 & 2.560 & 3.397 & 2.204 \\
Dif. & $4 \times 4$ & 192721 & 1.184574 & 167 & 10.199 & 2.356 & 3.129 & 2.092 \\
Dif. & $6 \times 6$ & 413449 & 1.184347 & 186 & 9.923 & 2.345 & 3.094 & 2.105 \\
Dif. & $8 \times 8$ & 717409 & 1.184132 & 204 & 9.683 & 2.381 & 3.097 & 2.158 \\
\hline
\end{tabular}
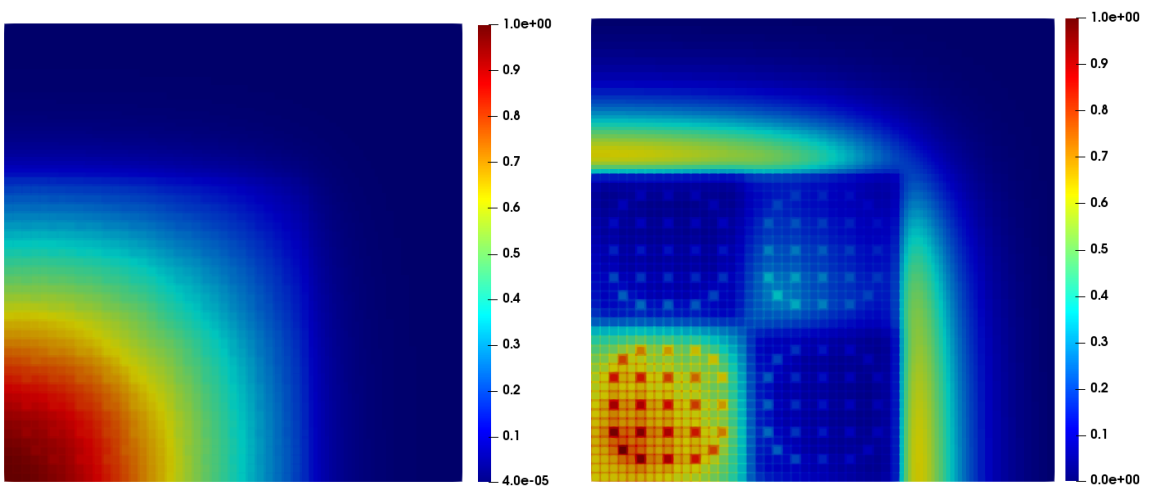

Figure 4.48: FDM Diffusion Flux 1st eigenvalue for energy groups 1 (left) and 7 (right).

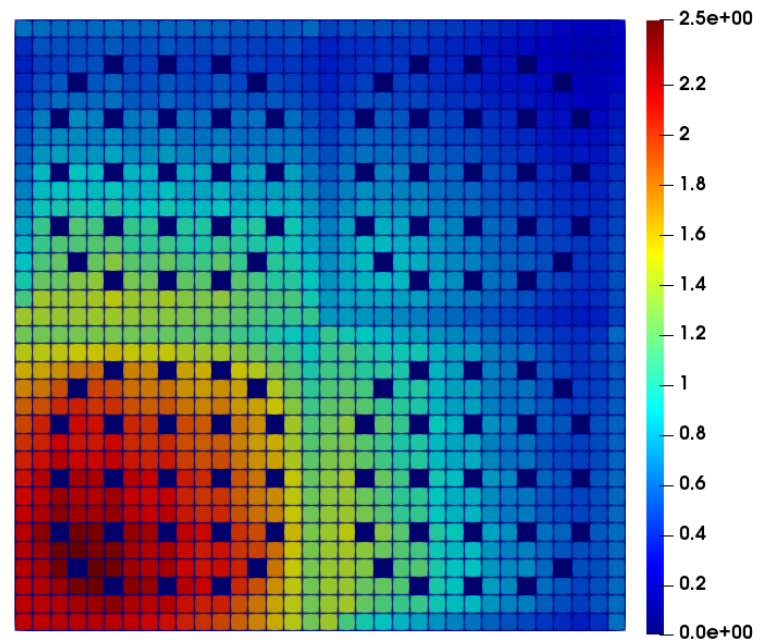

Figure 4.49: Power Distribution of C5G7 problem. 

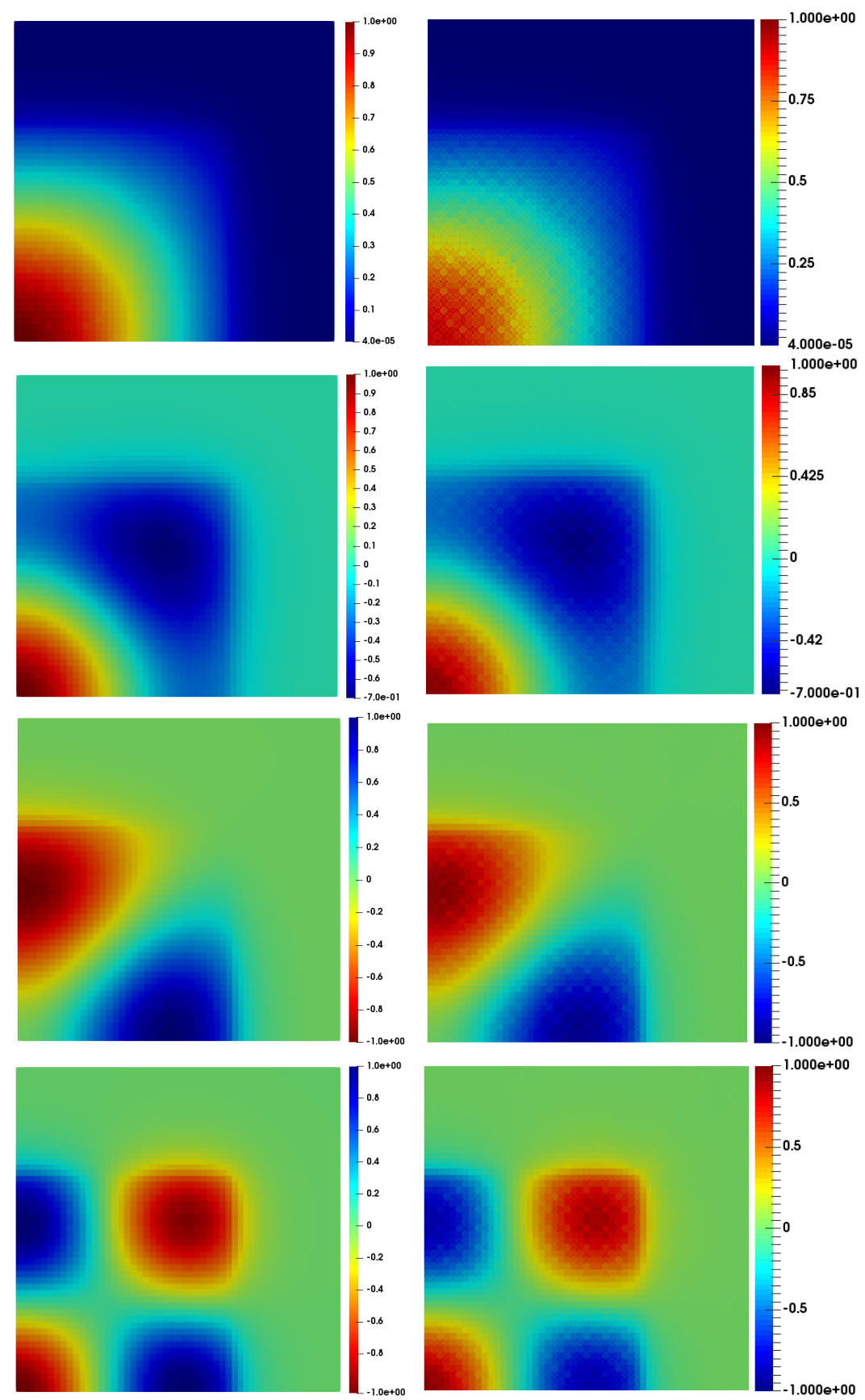

Figure 4.50: Eigenfluxes comparison for the four dominant eigenvalues of the $1^{\text {st }}$ energy group. FDM Diffusion on the left, FDM $S_{4}$ on the right. 
Table 4.11: C5G7 Test problem results. *Results from [Vidal-Ferràndiz et al., 2019].

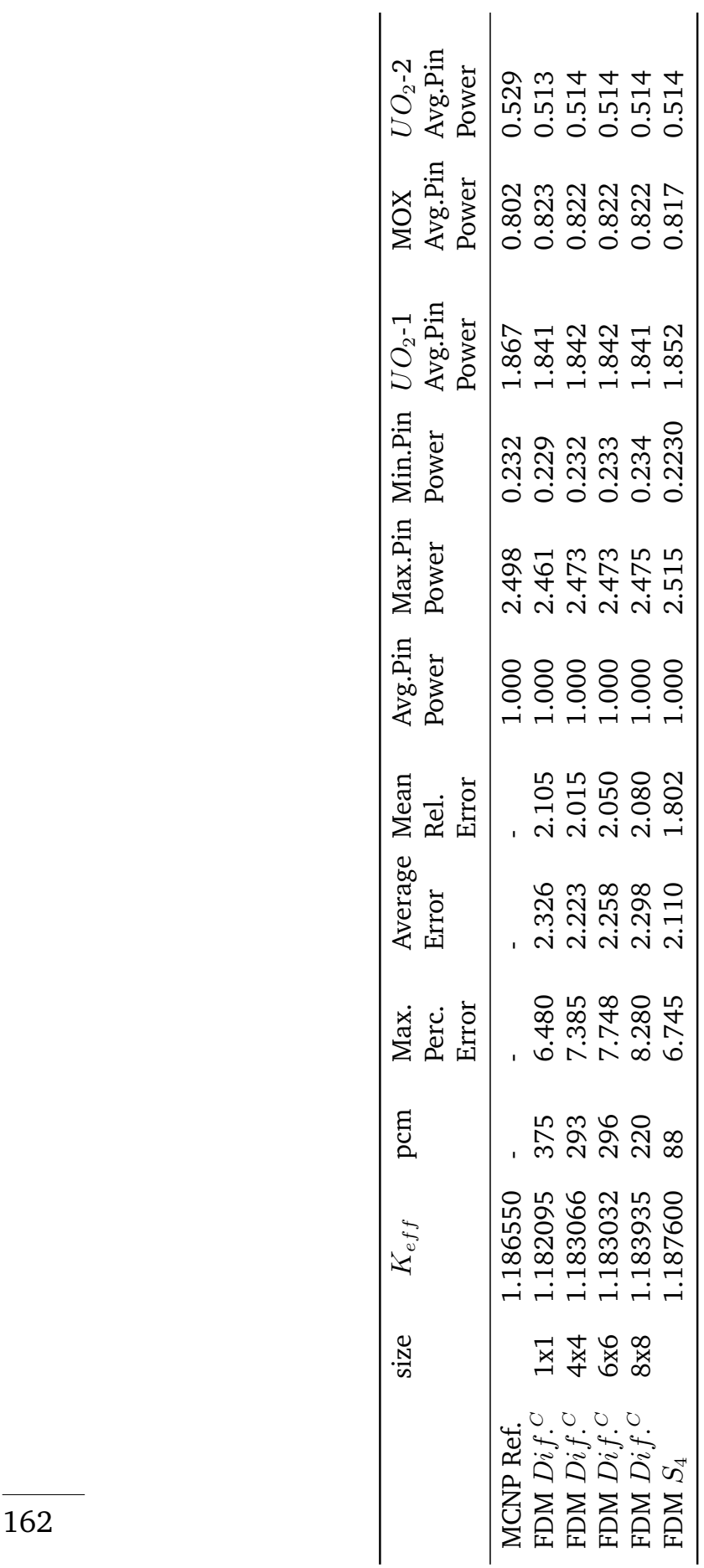




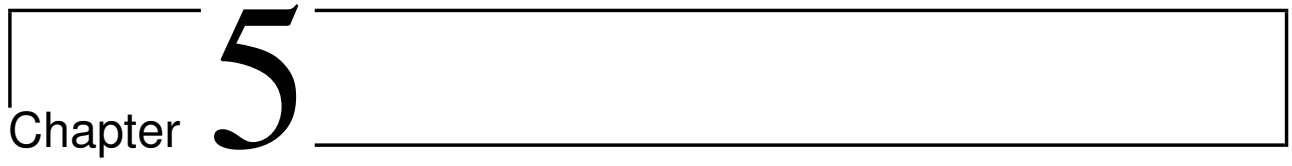

\section{Simplified Spherical Harmonics}

The methods explained in this chapter solve the Simplified Spherical Harmonics approximation to the neutron transport equation for $1 D$, $2 D$ and $3 D$ systems modeled with Cartesian geometry, by using the finite difference method for the spatial discretization. The method calculates the results for the steady-state equation by solving a generalized eigenvalue problem using a Krylov-Schur method. A formulation review of the Simplified Spherical Harmonics is explained, as well as, a study of the boundary conditions for different approaches of the finite difference method. The results calculated by this methodology are compared with the discrete ordinates and diffusion approximation methods explained in the previous chapters, all of them, using the same spatial discretization in order to show the different accuracy of each method without influence of the method used for discretizing the spatial variable.

\subsection{Introduction to the Simplified Spherical Harmonics}

The diffusion equation is widely used in the analysis and design of nuclear reactors, which allows core calculations with reasonable computational time and accuracy.

However the diffusion approximation is valid only under 4 assumptions. First, it is assumed that the neutron current is proportional to the neutron flux gradient. Second the medium is considered that have much less neutron absorption 
than scattering. Third, it is considered that the variation of the space neutron distribution is linear. Fourth, the scattering is assumed isotropic.

For calculations in which the reactor core is characterized as an homogeneous, isotropic and diffusive medium, diffusion approximation supply an accurate solution. Nevertheless, more detailed solutions, for example in pin level calculations, are desired for improved accuracy. In cases, where a control rod is considered, the highly absorbent material limits the applicability of the diffusion approximation. Therefore, more rigorous approximations for the neutron transport equation are required.

As seen previously, an accurate approach is to solve the neutron transport equation using the discrete ordinates method $\left(S_{N}\right)$. A review of this method was published by Lathrop and Carlson [1964]. At that time, the capabilities to solve complex problems with this methodology were limited, by the available computing power. Nowadays, the rapid progress of processors speed and the increase of the computer memory make possible the development of the $S_{N}$ codes capable of simulating more complex and realistic problems. However, even with the current computers and improved algorithms, realistic problem can only be solved on the largest computers

Another solution methodology uses the spherical harmonics $\left(P_{N}\right)$ approximation to the neutron transport equation. This approximation is developed by expanding the angular dependence of the flux into a set of spherical harmonic functions which can be combined naturally with the Legendre functions to handle the anisotropic scattering. Although it is necessary to use an infinite order of the spherical harmonics to have an exact solution, only spherical harmonics up to $\mathrm{N}$ are manageable for many analysis. The increase of the number of unknowns in multidimensional problems have to be taken into account. One dimensional planar geometry uses only $N+1$ number of equations for $P_{N}$ approximation. However, three-dimensional geometry needs $(N+1)^{2}$ number of equations making it relatively expensive to deal with. But defining even and odd parity fluxes the $P_{N}$ equations can be formulated as second order equations. However, the number of equations remains significant and angular moments, as well as, spatial derivatives are present in the coupling.

Since $P_{N}$ equations have relatively complexity, the simplified spherical harmonics approximation $\left(S P_{N}\right)$ was developed. This approximation was proposed by Gelbard [1960]. The idea is to replace the second derivatives in the one-dimensional planar geometry $P_{N}$ equations with a general three-dimensional Laplacian operator Brantley and Larsen [2000]. In this way, the number of the required equations by $S P_{N}$ approximation is fewer than $P_{N}$ equations and the resulting system of 
equations can be solved by most of the standard diffusion solvers. Another advantage of the $S P_{N}$ equations is that the problem which affects $S_{N}$ equations known as the "ray effect" is not present when the $S P_{N}$ equations are used.

However, the theoretical basis of the $S P_{N}$ equations is continuously being discussed because its solution does not normally converge to the transport solution when $N \rightarrow \infty$.

The $S P_{3}$ and $S P_{5}$ are commonly used and their results present much better accuracy with respect to the diffusion approximation in most of cases, giving results more similar to transport solution.

In this chapter, the author shows the discrepancies among the three approximations of the neutron transport equation presented in this thesis. These approximations are discrete ordinates method $\left(S_{N}\right)$, diffusion and simplified spherical harmonics when $N=3\left(S P_{3}\right)$. In order to perform a consistent comparison of the methods, all of them are formulated by using the finite difference method for the same spatial discretization.

\subsection{Derivation of the Simplified Spherical Harmonics Equations}

This section shows a review of the Simplified Spherical Harmonics $\left(S P_{N}\right)$ equations, in particular when $N=3$. Moreover, this section explains two different approaches of the finite difference method and the implementation of the Marshak boundary conditions.

Some different formulations to implement the Simplified $P_{N}$ equations have been published in several works. However, most of these do not include the details of the formulation of the boundary conditions for the finite difference method.

One of the most numerically and computationally efficient nomenclature is the one presented by Hamilton and Evans [2015]. However this work is based on the formulation developed by Brantley and Larsen [2000] which presents more understandable nomenclature with better physical interpretation.

The $S P_{3}$ steady state equations can be classically written as Tada et al. [2008]:

$-\nabla\left(D_{g} \nabla\left[\phi_{g}^{0}+2 \phi_{g}^{2}\right]\right)+\Sigma_{r, g}\left[\phi_{g}^{0}+2 \phi_{g}^{2}\right]=\frac{\chi_{g}}{k_{e f f}} \sum_{g^{\prime}} \nu \Sigma_{f, g^{\prime}} \phi_{g^{\prime}}^{0}+\sum_{g^{\prime} \neq g} \Sigma_{s, g^{\prime} \rightarrow g} \phi_{g^{\prime}}^{0}+2 \Sigma_{r, g} \phi_{g}^{2}$, 


$$
-\frac{27}{35} \nabla\left(D_{g} \nabla \phi_{g}^{2}\right)+\Sigma_{t, g} \phi_{g}^{2}=\frac{2}{5}\left\{\Sigma_{r, g} \phi_{g}^{0}-\left(\frac{\chi_{g}}{k_{e f f}} \sum_{g^{\prime}} \nu \Sigma_{f, g^{\prime}} \phi_{g^{\prime}}^{0}+\sum_{g^{\prime} \neq g} \Sigma_{s, g^{\prime} \rightarrow g} \phi_{g^{\prime}}^{0}\right)\right\} .
$$

In particular, for the one-dimensional case eqs.5.1 and 5.2 can be expressed as:

$$
\begin{aligned}
& \frac{\partial}{\partial x}\left(-D_{g}(x) \frac{\partial}{\partial x} \Phi_{g}(x)\right)+\Sigma_{r, g}(x) \Phi_{g}(x)= \\
& \quad \frac{\chi_{g}(x)}{K_{e f f}} \sum_{g^{\prime}} \nu \Sigma_{f, g^{\prime}}(x) \phi_{g^{\prime}}^{0}(x)+\sum_{g^{\prime} \neq g} \Sigma_{s, g^{\prime} \rightarrow g}(x) \phi_{g^{\prime}}^{0}(x)+2 \Sigma_{r, g}(x) \phi_{g}^{2}(x), \quad \text { (5.3) } \\
& \frac{27}{35} \frac{\partial}{\partial x}\left(-D_{g}(x) \frac{\partial}{\partial x} \phi_{g}^{2}(x)\right)+\Sigma_{t, g}(x) \phi_{g}^{2}(x)= \\
& \frac{2}{5}\left\{\Sigma_{r, g}(x) \phi_{g}^{0}(x)-\left(\frac{\chi_{g}(x)}{K_{e f f}} \sum_{g^{\prime}} \nu \Sigma_{f, g^{\prime}}(x) \phi_{g^{\prime}}^{0}(x)+\sum_{g^{\prime} \neq g} \Sigma_{s, g^{\prime} \rightarrow g}(x) \phi_{g^{\prime}}^{0}(x)\right)\right\} .
\end{aligned}
$$

Where

$$
\Phi_{i, g}=\phi_{i, g}^{0}+2 \phi_{i, g}^{2}
$$

$D_{g}$ : diffusion coefficient of group $g$

$\Sigma_{r, g}$ : removal cross section of group $g$ defined by the sumation of absorption and out-scatter cross section.

$$
\Sigma_{r, g}=\Sigma_{a, g}+\sum_{g^{\prime} \neq g} \Sigma_{s, g \rightarrow g^{\prime}}=\Sigma_{t, g}-\Sigma_{s, g \rightarrow g}
$$

$\chi_{g}:$ fission spectrum of group $g$

$k_{\text {eff }}:$ multiplication factor

$\nu \Sigma_{f, g}$ : fission production cross section of group $g$

$\Sigma_{s, g^{\prime} \rightarrow g}$ : scattering cross section from group $g^{\prime}$ to $g$

$\Sigma_{t, g}:$ total cross section of group $g$

$\phi_{g}^{m}$ : neutron flux of the $m$-th order moment in group $g$

Two approaches of the finite difference method are derived in the following sections. The first considers the flux in the center of each subdivision or cell. The second one, uses a edge-centered approach defining the unknowns on the boundary. The cell centered approach considers the cross sections in the middle of 
the cell while the edge-centered approach needs to use an average cross section. However, the authors find interesting to compare both approaches.

\subsubsection{Method 1: 1D Cell-centered Finite Difference Method}

Using the Fick's Law the current can be defined as:

$$
J_{g}^{0}(x)=-D_{g}^{0}(x) \frac{\partial \Phi_{g}(x)}{\partial x}, \quad D_{g}^{0}(x)=D_{g}(x) .
$$

So, Eq.5.3 can be reformulated as:

$$
\begin{aligned}
& \frac{\partial}{\partial x} J_{g}^{0}(x)+\Sigma_{r, g}(x) \Phi_{g}(x)= \\
& \quad \frac{\chi_{g}(x)}{k_{e f f}} \sum_{g^{\prime}} \nu \Sigma_{f, g^{\prime}}(x) \phi_{g^{\prime}}^{0}(x)+\sum_{g^{\prime} \neq g} \Sigma_{s, g^{\prime} \rightarrow g}(x) \phi_{g^{\prime}}^{0}(x)+2 \Sigma_{r, g}(x) \phi_{g}^{2}(x),
\end{aligned}
$$

Considering the cell-centered finite difference approach:

$$
\begin{aligned}
J_{g, i}^{0}-J_{g, i-1}^{0}+ & \int_{x_{i-1}}^{x_{i}} \Sigma_{r, g}(x) \Phi_{g}(x) d x=\frac{\chi_{g}(x)}{k_{e f f}} \int_{x_{i-1}}^{x_{i}} \sum_{g^{\prime}} \nu \Sigma_{f, g^{\prime}}(x) \phi_{g^{\prime}}^{0}(x) d x+ \\
& \int_{x_{i-1}}^{x_{i}} \sum_{g^{\prime} \neq g} \Sigma_{s, g^{\prime} \rightarrow g}(x) \phi_{g^{\prime}}^{0}(x) d x+2 \int_{x_{i-1}}^{x_{i}} \Sigma_{r, g}(x) \phi_{g}^{2}(x) d x,
\end{aligned}
$$

where:

$$
\begin{gathered}
\int_{x_{i-1}}^{x_{i}} \Sigma_{r, g}(x) \Phi_{g}(x) d x=\Sigma_{r, g}(x) \int_{x_{i-1}}^{x_{i}} \Phi_{g}(x) d x=\Sigma_{r, i, g} h_{i} \Phi_{i, g}, \\
\int_{x_{i-1}}^{x_{i}} \sum_{g^{\prime}} \nu \Sigma_{f, g^{\prime}}(x) \phi_{g^{\prime}}^{0}(x) d x=\sum_{g^{\prime}} \nu \Sigma_{f, i, g^{\prime}} h_{i} \phi_{i, g^{\prime}}^{0}, \\
\int_{x_{i-1}}^{x_{i}} \sum_{g^{\prime} \neq g} \Sigma_{s, g^{\prime} \rightarrow g}(x) \phi_{g^{\prime}}^{0}(x) d x=\sum_{g^{\prime} \neq g} \Sigma_{s, i, g^{\prime} \rightarrow g} h_{i} \phi_{i, g^{\prime}}^{0},
\end{gathered}
$$




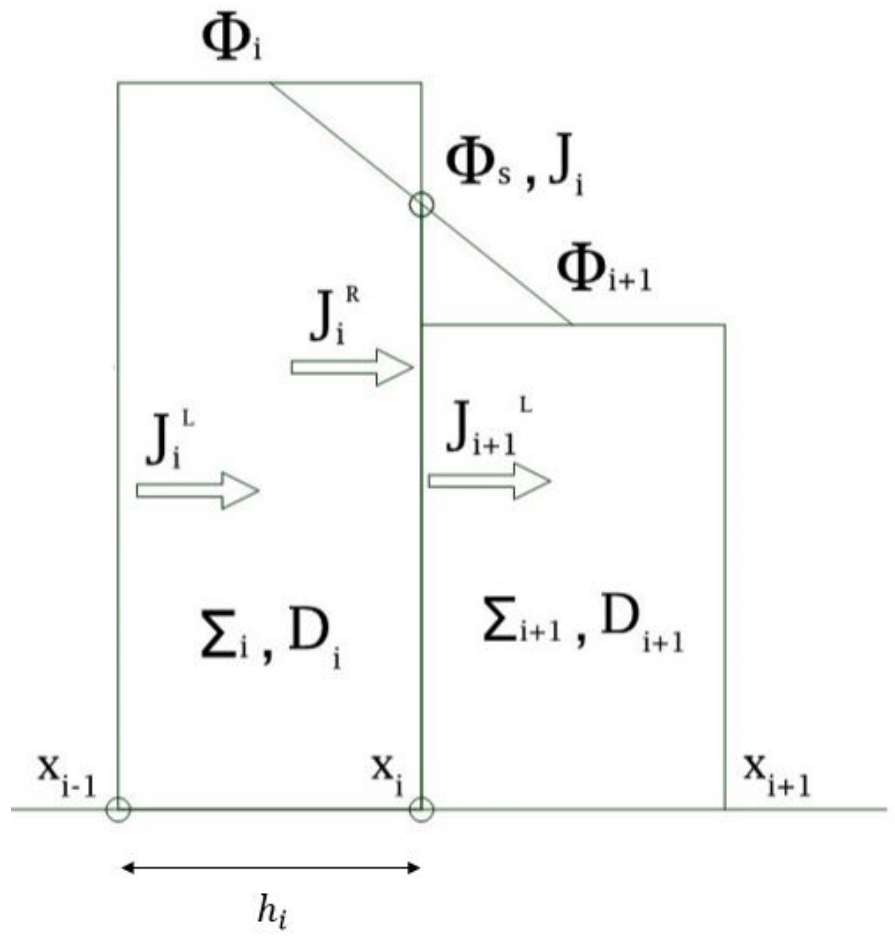

Figure 5.1: Cell-centered Finite Difference.

$$
\begin{gathered}
\int_{x_{i-1}}^{x_{i}} \Sigma_{r, g}(x) \phi_{g}^{2}(x) d x=\Sigma_{r, i, g} h_{i} \phi_{i, g}^{2}, \\
\Phi_{i, g}=\frac{1}{h_{i}} \int_{x_{i-1}}^{x_{i}} \Phi_{g}(x) d x
\end{gathered}
$$

This allows eq.5.6 to be written in the form of eq.5.13

$$
\begin{aligned}
& J_{i, g}^{0}-J_{i-1, g}^{0}+\Sigma_{r, i, g} h_{i} \Phi_{i, g}= \\
& \quad \frac{\chi_{i, g}}{k_{e f f}} \sum_{g^{\prime}} \nu \Sigma_{f, i, g^{\prime}} h_{i} \phi_{i, g^{\prime}}^{0}+\sum_{g^{\prime} \neq g} \Sigma_{s, i, g^{\prime} \rightarrow g} h_{i} \phi_{i, g^{\prime}}^{0}+2 \Sigma_{r, i, g} h_{i} \phi_{i, g}^{2},
\end{aligned}
$$


Taking into account the present cell-centered finite difference scheme we can define:

$$
\begin{gathered}
J_{i, g}^{0, R}(x)=-D_{i, g}^{0}(x) \frac{\partial \Phi_{g}(x)}{\partial x}, \\
J_{i, g}^{0, R}=-D_{i, g}^{0} \frac{\Phi_{i, g}^{R}-\Phi_{i, g}}{h_{i} / 2}, \\
J_{i+1, g}^{0, L}=-D_{i+1, g}^{0} \frac{\Phi_{i+1, g}-\Phi_{i+1, g}^{L}}{h_{i+1} / 2},
\end{gathered}
$$

with the interface conditions:

$$
J_{i}^{0, R}=J_{i+1}^{0, L}=J_{i}^{0},
$$

$$
\Phi_{i}^{R}=\Phi_{i+1}^{L}=\Phi_{s} .
$$

Then, from eq.5.17 :

$$
\begin{gathered}
-D_{i, g}^{0} \frac{\Phi_{s, g}-\Phi_{i, g}}{h_{i} / 2}=-D_{i+1, g}^{0} \frac{\Phi_{i+1, g}-\Phi_{s, g}}{h_{i+1} / 2}, \\
\Phi_{s, g}=\frac{D_{i, g}^{0} / h_{i}}{D_{i, g}^{0} / h_{i}+D_{i+1, g}^{0} / h_{i+1}} \Phi_{i, g}+\frac{D_{i+1, g}^{0} / h_{i+1}}{D_{i, g}^{0} / h_{i}+D_{i+1, g}^{0} / h_{i+1}} \Phi_{i+1, g}= \\
\omega_{i, g}^{0} \Phi_{i, g}+\left(1-\omega_{i, g}^{0}\right) \Phi_{i+1, g} .
\end{gathered}
$$

Then, substituting eq.5.20 into eq.5.15 the currents of the eq.5.13 can be expressed as:

$$
\begin{gathered}
J_{i, g}^{0}=J_{i, g}^{0, R}=-\widetilde{D}_{i, g}^{0}\left(\Phi_{i+1, g}-\Phi_{i, g}\right), \\
J_{i-1, g}^{0}=J_{i, g}^{0, L}=-\widetilde{D}_{i-1, g}^{0}\left(\Phi_{i, g}-\Phi_{i-1, g}\right),
\end{gathered}
$$

where:

$$
\widetilde{D}_{i, g}^{0}=\frac{2 D_{i, g}^{0} D_{i+1, g}^{0}}{D_{i, g}^{0} h_{i+1}+D_{i+1, g}^{0} h_{i}},
$$




$$
\widetilde{D}_{i-1, g}^{0}=\frac{2 D_{i-1, g}^{0} D_{i, g}^{0}}{D_{i-1, g}^{0} h_{i}+D_{i, g}^{0} h_{i-1}}
$$

Finally, eq.5.13 is re-written as a discretized mesh balance equation as follows:

$$
\begin{aligned}
-\widetilde{D}_{i-1, g}^{0} & \Phi_{i-1, g}+\left[\widetilde{D}_{i-1, g}^{0}+\widetilde{D}_{i, g}^{0}+\Sigma_{r, i, g} h_{i}\right] \Phi_{i, g}-\widetilde{D}_{i, g}^{0} \Phi_{i+1, g}= \\
& \frac{\chi_{i, g}}{k_{e f f}} \sum_{g^{\prime}} \nu \Sigma_{f, i, g^{\prime}} h_{i} \phi_{i, g^{\prime}}^{0}+\sum_{g^{\prime} \neq g} \Sigma_{s, i, g^{\prime} \rightarrow g} h_{i} \phi_{i, g^{\prime}}^{0}+2 \Sigma_{r, i, g} h_{i} \phi_{i, g}^{2} .
\end{aligned}
$$

The same procedure can be followed for the eq.5.4. Considering the Fick's Law:

$$
J_{g}^{2}(x)=-D_{g}^{2}(x) \frac{\partial \phi_{g}^{2}(x)}{\partial x}, \quad D_{g}^{2}(x)=\frac{27}{35} D_{g}(x) .
$$

Eq.5.4 takes the form:

$$
\begin{aligned}
& \frac{\partial}{\partial x} J_{g}^{2}(x)+\Sigma_{t, g}(x) \phi_{g}^{2}(x)= \\
& \frac{2}{5} \Sigma_{r, g}(x) \phi_{g}^{0}(x)-\frac{2}{5} \frac{\chi_{g}(x)}{k_{e f f}} \sum_{g^{\prime}} \nu \Sigma_{f, g^{\prime}}(x) \phi_{g^{\prime}}^{0}(x)-\frac{2}{5} \sum_{g^{\prime} \neq g} \Sigma_{s, g^{\prime} \rightarrow g}(x) \phi_{g^{\prime}}^{0}(x),
\end{aligned}
$$

using cell-centered finite difference approximation:

$$
\begin{aligned}
& J_{i, g}^{2}-J_{i-1, g}^{2}+\Sigma_{t, i, g} h_{i} \phi_{i, g}^{2}= \\
& \quad \frac{2}{5} \Sigma_{r, i, g} h_{i} \phi_{i, g}^{0}-\frac{2}{5} \frac{\chi_{i, g}}{k_{e f f}} \sum_{g^{\prime}} \nu \Sigma_{f, i, g^{\prime}} h_{i} \phi_{i, g^{\prime}}^{0}-\frac{2}{5} \sum_{g^{\prime} \neq g} \Sigma_{s, i, g^{\prime} \rightarrow g}(x) h_{i} \phi_{i, g^{\prime}}^{0} .
\end{aligned}
$$

Then, the currents of the eq.5.28 can be expressed as:

$$
\begin{gathered}
J_{i, g}^{2}=J_{i, g}^{2, R}=-\widetilde{D}_{i, g}^{2}\left(\phi_{i+1, g}^{2}-\phi_{i, g}^{2}\right), \\
J_{i-1, g}^{2}=J_{i, g}^{2, L}=-\widetilde{D}_{i-1, g}^{2}\left(\phi_{i, g}^{2}-\phi_{i-1, g}^{2}\right),
\end{gathered}
$$


where:

$$
\begin{gathered}
\widetilde{D}_{i, g}^{2}=\frac{2 D_{i, g}^{2} D_{i+1, g}^{2}}{D_{i, g}^{2} h_{i+1}+D_{i+1, g}^{2} h_{i}}, \\
\widetilde{D}_{i-1, g}^{2}=\frac{2 D_{i-1, g}^{2} D_{i, g}^{2}}{D_{i-1, g}^{2} h_{i}+D_{i, g}^{2} h_{i-1}} .
\end{gathered}
$$

Finally, eq.5.28 is re-written as a discretized mesh balance equation as follows:

$$
\begin{aligned}
& -\widetilde{D}_{i-1, g}^{2} \phi_{i-1, g}^{2}+\left[\widetilde{D}_{i-1, g}^{2}+\widetilde{D}_{i, g}^{2}+\Sigma_{t, i, g} h_{i}\right] \phi_{i, g}^{2}-\widetilde{D}_{i, g}^{2} \phi_{i+1, g}^{2}= \\
& \quad \frac{2}{5} \Sigma_{r, i, g} h_{i} \phi_{i, g}^{0}-\frac{2}{5} \frac{\chi_{i, g}}{k_{e f f}} \sum_{g^{\prime}} \nu \Sigma_{f, i, g^{\prime}} h_{i} \phi_{i, g^{\prime}}^{0}-\frac{2}{5} \sum_{g^{\prime} \neq g} \Sigma_{s, i, g^{\prime} \rightarrow g}(x) h_{i} \phi_{i, g^{\prime}}^{0} .
\end{aligned}
$$

Then the couple $S P_{3}$ equations discretized by using finite difference method are:

$$
\begin{aligned}
& -\widetilde{D}_{i-1, g}^{0} \Phi_{i-1, g}+\left[\widetilde{D}_{i-1, g}^{0}+\widetilde{D}_{i, g}^{0}+\Sigma_{r, i, g} h_{i}\right] \Phi_{i, g}-\widetilde{D}_{i, g}^{0} \Phi_{i+1, g}= \\
& \frac{\chi_{i, g}}{k_{e f f}} \sum_{g^{\prime}} \nu \Sigma_{f, i, g^{\prime}} h_{i} \phi_{i, g^{\prime}}^{0}+\sum_{g^{\prime} \neq g} \Sigma_{s, i, g^{\prime} \rightarrow g} h_{i} \phi_{i, g^{\prime}}^{0}+2 \Sigma_{r, i, g} h_{i} \phi_{i, g}^{2}, \\
& -\widetilde{D}_{i-1, g}^{2} \phi_{i-1, g}^{2}+\left[\widetilde{D}_{i-1, g}^{2}+\widetilde{D}_{i, g}^{2}+\Sigma_{t, i, g} h_{i}\right] \phi_{i, g}^{2}-\widetilde{D}_{i, g}^{2} \phi_{i+1, g}^{2}= \\
& \frac{2}{5} \Sigma_{r, i, g} h_{i} \phi_{i, g}^{0}-\frac{2}{5} \frac{\chi_{i, g}}{k_{e f f}} \sum_{g^{\prime}} \nu \Sigma_{f, i, g^{\prime}} h_{i} \phi_{i, g^{\prime}}^{0}-\frac{2}{5} \sum_{g^{\prime} \neq g} \Sigma_{s, i, g^{\prime} \rightarrow g}(x) h_{i} \phi_{i, g^{\prime}}^{0},
\end{aligned}
$$

where:

$$
\begin{gathered}
\widetilde{D}_{i, g}^{m}=\frac{2 D_{i, g}^{m} D_{i+1, g}^{m}}{D_{i, g}^{m} h_{i+1}+D_{i+1, g}^{m} h_{i}}, \\
\widetilde{D}_{i-1, g}^{m}=\frac{2 D_{i-1, g}^{m} D_{i, g}^{m}}{D_{i-1, g}^{m} h_{i}+D_{i, g}^{m} h_{i-1}},
\end{gathered}
$$




$$
\begin{aligned}
m & =0,2, \\
\Phi_{i, g} & =\phi_{i, g}^{0}+2 \phi_{i, g}^{2}, \\
D_{i, g}^{0} & =D_{i, g}=\frac{1}{3 \Sigma_{t, i, g}}, \\
D_{i, g}^{2} & =\frac{27}{35} D_{i, g} .
\end{aligned}
$$

\subsection{2 $S P_{3}$ boundary conditions applied to the cell-centered Scheme}

The $S P_{3}$ vacuum boundary conditions for these equations are given by (Larsen et al. [1996], Brantley and Larsen [2000], Hamilton and Evans [2015]). The well known Marshak-like boundary conditions for vacuum can be expressed as:

$$
\begin{gathered}
D_{i, g}^{0} \vec{n} \cdot \nabla \Phi_{i, g}+\frac{1}{2} \phi_{i, g}^{0}+\frac{5}{8} \phi_{i, g}^{2}=0, \\
D_{i, g}^{2} \vec{n} \cdot \nabla \phi_{i, g}^{2}-\frac{3}{40} \phi_{i, g}^{0}+\frac{3}{8} \phi_{i, g}^{2}=0,
\end{gathered}
$$

Where:

$$
\begin{aligned}
& \Phi_{i, g}=\phi_{i, g}^{0}+2 \phi_{i, g}^{2} \\
& D_{i, g}^{0}=D_{i, g}=\frac{1}{3 \Sigma_{t, i, g}} \\
& D_{i, g}^{2}=\frac{27}{35} D_{i, g}=\frac{9}{35 \Sigma_{t, i, g}} .
\end{aligned}
$$

Marshak boundary conditions are easy to implement in a Finite Difference edgecentered approach or finite element method where some unknowns are the points considered at the boundary interface. However, considering the finite difference cell-centered approach, it is necessary to define the flux at the boundary in terms of the flux in the cell. For this purpose, three different approaches are considered in the next section. 
The $S P_{3}$ reflective boundary conditions are more straightforward. These can be easily obtained as:

$$
\begin{aligned}
& \nabla \Phi_{i, g}=0, \\
& \nabla \phi_{i, g}^{2}=0 .
\end{aligned}
$$

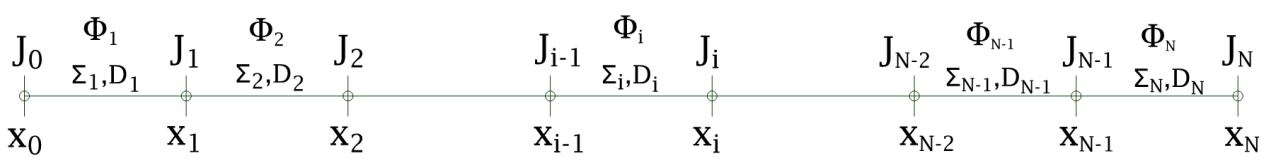

Figure 5.2: Cell-centered finite difference scheme.

First vacuum B.C. approach: $\phi_{x_{0}, g}^{0}=\phi_{1, g}^{0}$ and $\phi_{x_{0}, g}^{2}=\phi_{1, g}^{2}$

This approach assumes that the flux on the left boundary is the same as the flux in the first cell. Then, the eqs.5.42 and 5.43 can be introduced into the balance equations 5.13 and 5.28. This approach is not recommended. Depending on the problem, the solution obtained by using this approach could be more different with respect to a finely meshed transport. One simple solution to obtain good results in these cases with this calculation is to use a finer mesh close to the boundary.

$$
\begin{aligned}
& J_{x_{0}, g}^{0}=-\frac{1}{2} \phi_{1, g}^{0}-\frac{5}{8} \phi_{1, g}^{2} \\
& J_{x_{0}, g}^{2}=\frac{3}{40} \phi_{1, g}^{0}-\frac{3}{8} \phi_{1, g}^{2} .
\end{aligned}
$$


Second vacuum B.C. approach

This approach provides better results than the previous one. The boundary conditions are defined in a similar way to the diffusion equations, but only for the first moment $\phi_{x_{0}, g}^{0}$. We can obtain the $P_{1}$ equation starting from $S P_{3}$ equations by removing the second $S P_{3}$ equation for the $2^{\text {nd }}$ moment (Eq.5.2) and by removing $\phi_{g}^{2}$ inside the first $S P_{3}$ equation for the zero moment (Eq.5.1). We can do the same with the boundary conditions. So, the balance equation is:

$$
-D_{g} \nabla^{2} \phi_{g}^{0}+\Sigma_{r, g} \phi_{g}^{0}=\frac{\chi_{g}}{K_{e f f}} \sum_{g^{\prime}} \nu \Sigma_{f, g^{\prime}} \phi_{g^{\prime}}^{0}+\sum_{g^{\prime} \neq g} \Sigma_{s, g^{\prime} \rightarrow g} \phi_{g^{\prime}}^{0},
$$

The left boundary condition equation used in the diffusion equation where $\alpha=$ $1 / 2$ for the vacuum condition is:

$$
\begin{gathered}
J_{x_{0}, g}=-\alpha_{L} \cdot \phi_{x_{0}, g}=-D_{1} \frac{\phi_{1, g}-\phi_{x_{0}, g}}{h_{1} / 2}, \\
J_{x_{0}, g}=-2 \frac{\left(D_{1, g} / h_{1}\right)\left(\alpha_{L} / 2\right)}{\left[\left(D_{1, g} / h_{1}\right)+\left(\alpha_{L} / 2\right)\right]} \phi_{1, g}=-\widetilde{D}_{0, g} \phi_{1, g},
\end{gathered}
$$

with $\alpha_{L}=1 / 2$ we have the exact vacuum boundary condition for $P_{1}$ (Diffusion) equation:

$$
J_{x_{0}, g}=-\frac{1}{2} \frac{\left(D_{1, g} / h_{1}\right)}{\left[\left(D_{1, g} / h_{1}\right)+(1 / 4)\right]} \phi_{1, g}
$$

Taking this into account, one could do something similar for the $S P_{3}$ equations, but in this case the solution for the boundary condition is not so straighforward, due to the second moment flux. It can be appreciated that the next equation corresponds to the eq.5.45, but for the first $S P_{3}$ equation:

$$
J_{x_{0}, g}^{0}=-\alpha_{L} \cdot \phi_{x_{0}, g}^{0}-\beta_{L} \cdot \phi_{0, g}^{2, L}=-D_{1, g} \frac{\phi_{1, g}^{0}+2 \phi_{1, g}^{2}-\phi_{x_{0}, g}^{0}-2 \phi_{x_{0}, g}^{2}}{h_{1} / 2},
$$

For obtaining a more simple solution, one can approximate the $S P_{3}$ boundary conditions by adding the term of the Eq.5.47 defined for $P_{1}$ equation into the $S P_{3}$ first moment boundary condition equation. 


$$
\begin{gathered}
J_{x_{0}, g}^{0}=-\frac{1}{2} \frac{\left(D_{1, g} / h_{1}\right)}{\left[\left(D_{1, g} / h_{1}\right)+(1 / 4)\right]} \phi_{1, g}^{0}-\frac{5}{8} \phi_{1, g}^{2} \\
J_{x_{0}, g}^{2}=\frac{3}{40} \phi_{1, g}^{0}-\frac{3}{8} \phi_{1, g}^{2} .
\end{gathered}
$$

Although this is not the exact solution the numerical results are significantly better than those obtained with the previous simplification.

Third approach: Exact vacuum B.C. for SP3

This section defines the exact boundary conditions for $S P_{3}$ using the cell-centered finite difference. Starting from eqs.5.38 and 5.39 and the Fick's law, the left boundary equations are:

$$
\begin{aligned}
& J_{x_{0}, g}^{0}=-\frac{1}{2} \phi_{x_{0}, g}^{0}-\frac{5}{8} \phi_{x_{0}, g}^{2}, \\
& J_{x_{0}, g}^{2}=\frac{3}{40} \phi_{x_{0}, g}^{0}-\frac{3}{8} \phi_{x_{0}, g}^{2},
\end{aligned}
$$

In order to have the same number of unknowns and equations, one need to define eqs.5.51 and 5.52 in terms of $\phi_{1, g}^{0}$ and $\phi_{1, g}^{2}$. To do that, it can be defined eqs. 5.53 and 5.54 .

$$
\begin{gathered}
J_{x_{0}, g}^{0}=-\frac{D_{1, g}^{0}}{h_{1} / 2}\left[\Phi_{1, g}-\Phi_{x_{0}, g}\right]=-\frac{D_{1, g}^{0}}{h_{1} / 2}\left[\left(\phi_{1, g}^{0}+2 \phi_{1, g}^{2}\right)-\left(\phi_{x_{0}, g}^{0}+2 \phi_{x_{0}, g}^{2}\right)\right], \\
J_{x_{0}, g}^{2}=-\frac{27}{35} \cdot \frac{D_{1, g}^{0}}{h_{1} / 2}\left[\phi_{1, g}^{2}-\phi_{x_{0}, g}^{2}\right],
\end{gathered}
$$

Eqs.5.51 and 5.52 will now be solved for $\phi_{x_{0}, g}^{0}$ and $\phi_{x_{0}, g}^{2}$ in terms of $J_{x_{0}, g}^{0}$ and $J_{x_{0}, g}^{2}$. Multiplying eq.5.52 by $20 / 3$ : 


$$
\frac{20}{3} J_{x_{0}, g}^{2}-\frac{1}{2} \phi_{x_{0}, g}^{0}+\frac{5}{2} \phi_{x_{0}, g}^{2}=0,
$$

Adding this to eq. 5.51 gives:

$$
J_{x_{0}, g}^{0}+\frac{20}{3} J_{x_{0}, g}^{2}+\left[\frac{5}{8}+\frac{5}{2}\right] \phi_{x_{0}, g}^{2}=0
$$

So,

$$
\phi_{x_{0}, g}^{2}=-\frac{8}{25} J_{x_{0}, g}^{0}-\frac{32}{15} J_{x_{0}, g}^{2},
$$

Introducing this result into eq. 5.51.

$$
\phi_{x_{0}, g}^{0}=-\frac{8}{5} J_{x_{0}, g}^{0}+\frac{8}{3} J_{x_{0}, g}^{2},
$$

Introducing eqs. 5.55 and 5.56 into eqs. 5.53 and 5.54:

$$
\begin{gathered}
J_{x_{0}, g}^{0}=-\frac{2 D_{1, g}^{0}}{a h_{1}}\left[\phi_{1, g}^{0}+2 \phi_{1, g}^{2}+\frac{8}{5} J_{x_{0}, g}^{2}\right], \\
J_{x_{0}, g}^{2}=-\frac{27}{35} \cdot \frac{2 D_{1, g}^{0}}{b h_{1}}\left[\phi_{1, g}^{2}+\frac{8}{25} J_{x_{0}, g}^{0}\right],
\end{gathered}
$$

where:

$$
a=\left[1+\frac{112}{25} \cdot \frac{D_{1, g}^{0}}{h_{1}}\right], \quad b=\left[1+\frac{27}{35} \cdot \frac{32}{15} \cdot \frac{2 D_{1, g}^{0}}{h_{1}}\right],
$$

Introducing eq.5.58 into eq.5.57 we get:

$$
J_{x_{0}, g}^{0}=-\frac{2 D_{1, g}^{0}}{a c h_{1}} \phi_{1, g}^{0}-\frac{4 D_{1, g}^{0}}{a c h_{1}}\left(1-\frac{216}{175} \cdot \frac{D_{1, g}^{0}}{b h_{1}}\right) \phi_{1, g}^{2},
$$


where:

$$
c=\left[1-\frac{2 D_{1, g}^{0}}{a h_{1}}\left(\frac{1728}{4375} \cdot \frac{2 D_{1, g}^{0}}{b h_{1}}\right)\right]
$$

Introducing eq.5.59 into eq.5.58 we get:

$$
J_{x_{0}, g}^{2}=-\frac{27}{35} \cdot \frac{2 D_{1, g}^{0} e}{b h_{1}} \phi_{1, g}^{0}-\frac{27}{35} \cdot \frac{2 D_{1, g}^{0} d}{b h_{1}} \phi_{1, g}^{2},
$$

where:

$$
d=\left[1-\frac{8}{25} \cdot \frac{4 D_{1, g}^{0}}{a c h_{1}}\left(1-\frac{216}{175} \cdot \frac{D_{1, g}^{0}}{b h_{1}}\right)\right], \quad e=\left[-\frac{8}{25} \cdot \frac{D_{1, g}^{0}}{a c h_{1}}\right]
$$

Eqs. 5.59 and 5.60 can be introduced into the balance equations 5.13 and 5.28 to apply vacuum boundary conditions.

Reflective and Zero flux boundary condition for $\mathrm{SP}_{3}$

The reflective boundary conditions are:

$$
J_{x_{0}, g}^{0}=0, \quad \text { and } \quad J_{x_{0}, g}^{2}=0,
$$

Zero flux boundary condition means that $\phi_{x_{0}, g}^{0}=0$ and $\phi_{x_{0}, g}^{2}=0$ into equations 5.53 and 5.54 .

$$
J_{x_{0}, g}^{0}=-\frac{D_{1, g}^{0}}{h_{1} / 2}\left(\phi_{1, g}^{0}+2 \phi_{1, g}^{2}\right), \quad \text { and } \quad J_{x_{0}, g}^{2}=-\frac{27}{35} \cdot \frac{D_{1, g}^{0}}{h_{1} / 2} \phi_{1, g}^{2} .
$$




\subsubsection{Method 2: 1D Edge-centered Finite Difference Method}

Starting from the eq.5.6:

$$
\begin{aligned}
& \frac{\partial}{\partial x} J_{g}^{0}(x)+\Sigma_{r, g}(x) \Phi_{g}(x)= \\
& \quad \frac{\chi_{g}(x)}{K_{e f f}} \sum_{g^{\prime}} \nu \Sigma_{f, g^{\prime}}(x) \phi_{g^{\prime}}^{0}(x)+\sum_{g^{\prime} \neq g} \Sigma_{s, g^{\prime} \rightarrow g}(x) \phi_{g^{\prime}}^{0}(x)+2 \Sigma_{r, g}(x) \phi_{g}^{2}(x),
\end{aligned}
$$

and using finite difference edge-centered scheme approximation according to Fig.5.3:

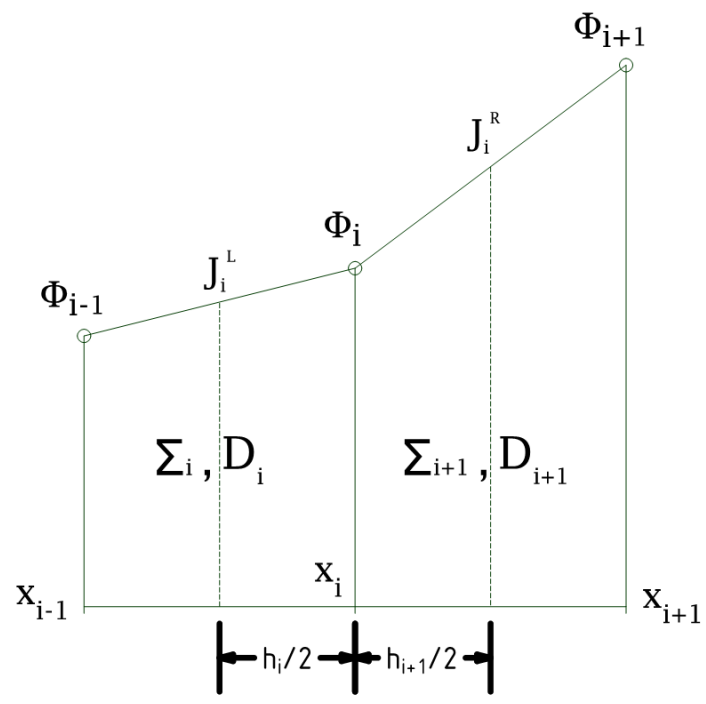

Figure 5.3: Finite difference edge-centered scheme.

$$
\begin{gathered}
J_{g, i}^{0, R}-J_{g, i}^{0, L}+\int_{x_{i-1}+h_{i} / 2}^{x_{i}+h_{i+1} / 2} \Sigma_{r, g}(x) \Phi_{g}(x) d x=\frac{\chi_{g}(x)}{k_{e f f}} \int_{x_{i-1}+h_{i} / 2}^{x_{i}+h_{i+1} / 2} \sum_{g^{\prime}} \nu \Sigma_{f, g^{\prime}}(x) \phi_{g^{\prime}}^{0}(x) d x+ \\
\int_{x_{i-1}+h_{i} / 2}^{x_{i}+h_{i+1} / 2} \sum_{g^{\prime} \neq g} \Sigma_{s, g^{\prime} \rightarrow g}(x) \phi_{g^{\prime}}^{0}(x) d x+2 \int_{x_{i-1}+h_{i} / 2}^{x_{i}+h_{i+1} / 2} \Sigma_{r, g}(x) \phi_{g}^{2}(x) d x, \quad \text { (5.61) }
\end{gathered}
$$




$$
\begin{aligned}
& \int_{x_{i-1}+h_{i} / 2}^{x_{i}+h_{i+1} / 2} \Sigma_{r, g}(x) \Phi_{g}(x) d x \approx \Phi_{g}\left(x_{i}\right) \int_{x_{i-1}+h_{i} / 2}^{x_{i}+h_{i+1} / 2} \Sigma_{r, g}(x) d x= \\
& \frac{1}{2}\left(h_{i} \Sigma_{r, g, i}+h_{i+1} \Sigma_{r, g, i+1}\right) \Phi_{g, i}=\bar{\Sigma}_{r, g, i} \Phi_{g, i}, \\
& \int_{x_{i-1}+h_{i} / 2}^{x_{i}+h_{i+1} / 2} \sum_{g^{\prime}} \nu \Sigma_{f, g^{\prime}}(x) \phi_{g^{\prime}}^{0}(x) d x \approx \sum_{g^{\prime}} \frac{1}{2}\left(h_{i} \nu \Sigma_{f, i, g^{\prime}}+h_{i+1} \nu \Sigma_{f, i+1, g^{\prime}}\right) \phi_{i, g^{\prime}}^{0} \\
& =\sum_{g^{\prime}} \overline{\nu \Sigma}_{f, g, i} \phi_{i, g^{\prime}}^{0}, \\
& \int_{x_{i-1}+h_{i} / 2}^{x_{i}+h_{i+1} / 2} \sum_{g^{\prime} \neq g} \Sigma_{s, g^{\prime} \rightarrow g}(x) \phi_{g^{\prime}}^{0}(x) d x \approx \sum_{g^{\prime} \neq g} \frac{1}{2}\left(h_{i} \Sigma_{s, g^{\prime} \rightarrow g, i}+h_{i+1} \Sigma_{s, g^{\prime} \rightarrow g, i+1}\right) \phi_{g^{\prime}, i}^{0} \\
& =\sum_{g^{\prime} \neq g} \bar{\Sigma}_{s, g^{\prime} \rightarrow g, i} \phi_{g^{\prime}, i}^{0}, \\
& \int_{x_{i-1}+h_{i} / 2}^{x_{i}+h_{i+1} / 2} \Sigma_{r, g}(x) \phi_{g}^{2}(x) d x \approx \frac{1}{2}\left(h_{i} \Sigma_{r, i, g}+h_{i+1} \Sigma_{r, i+1, g}\right) \phi_{i, g}^{2}=\bar{\Sigma}_{r, g, i} \phi_{g, i}^{2} .
\end{aligned}
$$

Eq.5.6 is transformed into Eq.5.66

$$
\begin{gathered}
J_{g, i}^{0, R}-J_{g, i}^{0, L}+\bar{\Sigma}_{r, g, i} \Phi_{g, i}= \\
\frac{\chi_{g, i}}{k_{e f f}} \sum_{g^{\prime}} \bar{\nu}_{f, g^{\prime}, i} \phi_{g^{\prime}, i}^{0}+\sum_{g^{\prime} \neq g} \bar{\Sigma}_{s, g^{\prime} \rightarrow g, i} \phi_{g^{\prime}, i}^{0}+2 \bar{\Sigma}_{r, g, i} \phi_{g, i}^{2}, \\
J_{g, i}^{0, R}(x)=-D_{g, i}^{0}(x) \frac{\partial \Phi_{g}(x)}{\partial x}, \\
J_{g, i}^{0, R}=-D_{g, i+1}^{0} \frac{\Phi_{g, i+1}-\Phi_{g, i}}{h_{i+1} / 2} \\
J_{g, i}^{0, L}=-D_{g, i}^{0} \frac{\Phi_{g, i}-\Phi_{g, i-1}}{h_{i} / 2} .
\end{gathered}
$$


Finally, Eq.5.66 is re-written as a discretized mesh balance equation as follows:

$$
\begin{gathered}
{\left[\frac{D_{g, i+1}^{0}}{h_{i+1}}+\frac{D_{g, i}^{0}}{h_{i}}+\bar{\Sigma}_{r, g, i}\right] \Phi_{g, i}-\frac{D_{g, i+1}^{0}}{h_{i+1}} \Phi_{g, i+1}-\frac{D_{g, i}^{0}}{h_{i}} \Phi_{g, i-1}-2 \bar{\Sigma}_{r, g, i} \phi_{g, i}^{2}=} \\
\frac{\chi_{g, i}}{k_{e f f}} \sum_{g^{\prime}} \bar{\nu}_{f, g^{\prime}, i} \phi_{g^{\prime}, i}^{0}+\sum_{g^{\prime} \neq g} \bar{\Sigma}_{s, g^{\prime} \rightarrow g, i} \phi_{g^{\prime}, i}^{0},
\end{gathered}
$$

Using the same procedure Eq.5.27 can be expressed in a discretized way as:

$$
\begin{aligned}
{\left[\frac{D_{g, i+1}^{2}}{h_{i+1}}+\frac{D_{g, i}^{2}}{h_{i}}+\bar{\Sigma}_{t, g, i}\right] } & \phi_{g, i}^{2}-\frac{D_{g, i+1}^{2}}{h_{i+1}} \phi_{g, i+1}^{2}-\frac{D_{g, i}^{2}}{h_{i}} \phi_{g, i-1}^{2}-\frac{2}{5} \bar{\Sigma}_{r, g, i} \phi_{g, i}^{0}= \\
& -\frac{2}{5} \frac{\chi_{g, i}}{k_{e f f}} \sum_{g^{\prime}} \overline{\nu \Sigma}_{f, g^{\prime}, i} \phi_{g^{\prime}, i}^{0}-\frac{2}{5} \sum_{g^{\prime} \neq g} \bar{\Sigma}_{s, g^{\prime} \rightarrow g, i} \phi_{g^{\prime}, i}^{0} .
\end{aligned}
$$

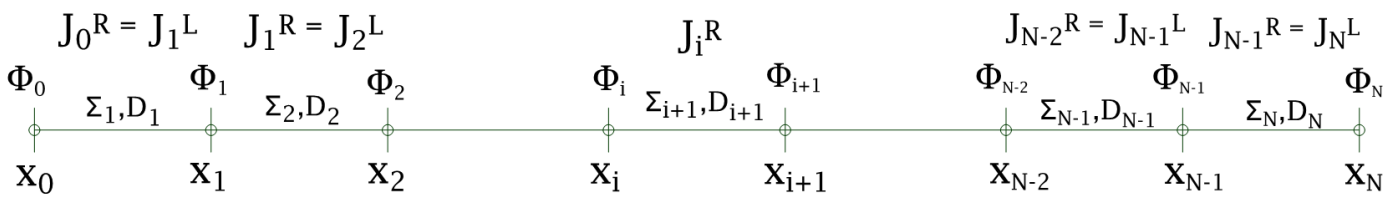

Figure 5.4: Edge-centered Scheme

Left vacuum boundary condition $(i=0)$

The definition of the boundary condition for the edge-centered scheme is more straightforward than for the cell-centered case. It is enough to consider the normal Marshak condition for the $S P_{3}$ applied to the boundary points.

Considering the Fick's Law and $\vec{n}=-1$ on the left boundary, one can write Eq.5.38 and 5.39 as:

$$
J_{g, 0}^{0}=-\frac{1}{2} \phi_{g, 0}^{0}-\frac{5}{8} \phi_{g, 0}^{2}
$$




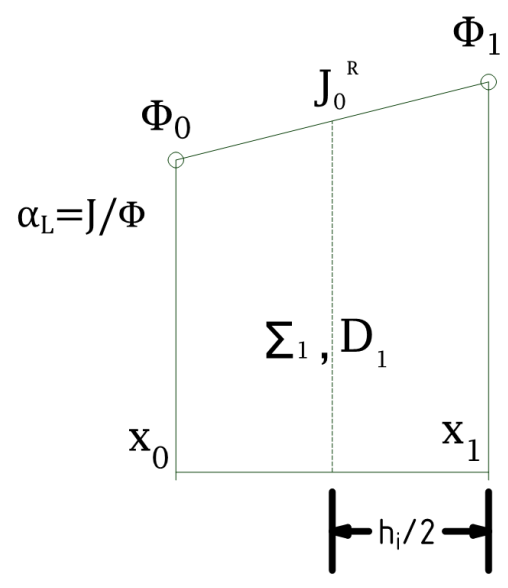

Figure 5.5: Left boundary conditions edge-centered scheme.

$$
J_{g, 0}^{2}=\frac{3}{40} \phi_{g, 0}^{0}-\frac{3}{8} \phi_{g, 0}^{2}
$$

Introducing Eq.5.72 and 5.68 into the balance equation Eq.5.66 we have the Eq.5.74 when $i=0$ :

$$
\begin{gathered}
{\left[\frac{D_{g, i+1}^{0}}{h_{i+1}}+\bar{\Sigma}_{r, g, i}\right] \Phi_{g, i}+\left(\frac{1}{2} \phi_{g, i}^{0}+\frac{5}{8} \phi_{g, i}^{2}\right)-\frac{D_{g, i+1}^{0}}{h_{i+1}} \Phi_{g, i+1}-2 \bar{\Sigma}_{r, g, i} \phi_{g, i}^{2}=} \\
\frac{\chi_{g, i}}{k_{e f f}} \sum_{g^{\prime}} \bar{\nu}_{f, g^{\prime}, i} \phi_{g^{\prime}, i}^{0}+\sum_{g^{\prime} \neq g} \bar{\Sigma}_{s, g^{\prime} \rightarrow g, i} \phi_{g^{\prime}, i}^{0},
\end{gathered}
$$

And introducing eq.5.73 into the balance equation for the second moment, one have eq.5.75 when $i=0$ :

$$
\begin{aligned}
{\left[\frac{D_{g, i+1}^{2}}{h_{i+1}}+\bar{\Sigma}_{t, g, i}\right] \phi_{g, i}^{2} } & +\left(\frac{-3}{40} \phi_{g, i}^{0}+\frac{3}{8} \phi_{g, i}^{2}\right)-\frac{D_{g, i+1}^{2}}{h_{i+1}} \phi_{g, i+1}^{2}-\frac{2}{5} \bar{\Sigma}_{r, g, i} \phi_{g, i}^{0}= \\
& -\frac{2}{5} \frac{\chi_{g, i}}{k_{e f f}} \sum_{g^{\prime}} \bar{\nu}_{f, g^{\prime}, i} \phi_{g^{\prime}, i}^{0}-\frac{2}{5} \sum_{g^{\prime} \neq g} \bar{\Sigma}_{s, g^{\prime} \rightarrow g, i,} \phi_{g^{\prime}, i}^{0} .
\end{aligned}
$$


Right vacuum boundary condition $(i=N)$

Using the same procedure than left boundary but with $\vec{n}=1$ :

$$
\begin{gathered}
J_{g, N}^{0}=\frac{1}{2} \phi_{g, N}^{0}+\frac{5}{8} \phi_{g, N}^{2}, \\
J_{g, N}^{2}=-\frac{3}{40} \phi_{g, N}^{0}+\frac{3}{8} \phi_{g, N}^{2},
\end{gathered}
$$

Introducing Eq.5.76 into the balance equation 5.66, one have the eq.5.78 when $i=N$ :

$$
\begin{array}{r}
{\left[\frac{D_{g, i}^{0}}{h_{i}}+\bar{\Sigma}_{r, g, i}\right] \Phi_{g, i}+\left(\frac{1}{2} \phi_{g, N}^{0}+\frac{5}{8} \phi_{g, N}^{2}\right)-\frac{D_{g, i}^{0}}{h_{i}} \Phi_{g, i-1}-2 \bar{\Sigma}_{r, g, i} \phi_{g, i}^{2}=} \\
\frac{\chi_{g, i}}{k_{e f f}} \sum_{g^{\prime}} \bar{\nu}_{f, g^{\prime}, i} \phi_{g^{\prime}, i}^{0}+\sum_{g^{\prime} \neq g} \bar{\Sigma}_{s, g^{\prime} \rightarrow g, i} \phi_{g^{\prime}, i}^{0},
\end{array}
$$

And introducing eq.5.77 into the balance equation for the second moment we have eq.5.79 when $i=N$ :

$$
\begin{aligned}
{\left[\frac{D_{g, i}^{2}}{h_{i}}+\bar{\Sigma}_{t, g, i}\right] \phi_{g, i}^{2}+} & \left(\frac{-3}{40} \phi_{g, N}^{0}+\frac{3}{8} \phi_{g, N}^{2}\right)-\frac{D_{g, i}^{2}}{h_{i}} \phi_{g, i-1}^{2}-\frac{2}{5} \bar{\Sigma}_{r, g, i} \phi_{g, i}^{0}= \\
& -\frac{2}{5} \frac{\chi_{g, i}}{k_{e f f}} \sum_{g^{\prime}} \overline{\nu \Sigma}_{f, g^{\prime}, i} \phi_{g^{\prime}, i}^{0}-\frac{2}{5} \sum_{g^{\prime} \neq g} \bar{\Sigma}_{s, g^{\prime} \rightarrow g, i} \phi_{g^{\prime}, i}^{0} .
\end{aligned}
$$




\subsection{D Cell centered scheme $S P_{3}$ Equations}

Starting with eq.5.1 and 5.2, two dimensional $S P_{3}$ equations can be written as:

$$
\begin{gathered}
-D_{g}(x, y) \frac{\partial^{2}}{\partial x^{2}} \Phi_{g}(x, y)-D_{g}(x, y) \frac{\partial^{2}}{\partial y^{2}} \Phi_{g}(x, y)+\Sigma_{r, g}(x, y) \Phi_{g}(x, y)= \\
\frac{\chi_{g}(x, y)}{k_{e f f}} \sum_{g^{\prime}} \nu \Sigma_{f, g^{\prime}}(x, y) \phi_{g^{\prime}}^{0}(x, y)+\sum_{g^{\prime} \neq g} \Sigma_{s, g^{\prime} \rightarrow g}(x, y) \phi_{g^{\prime}}^{0}(x, y)+2 \Sigma_{r, g}(x, y) \phi_{g}^{2}(x, y)
\end{gathered}
$$

$$
\begin{gathered}
-D_{g}(x, y) \frac{27}{35}\left[\frac{\partial^{2}}{\partial x^{2}} \phi_{g}^{2}(x, y)+\frac{\partial^{2}}{\partial y^{2}} \phi_{g}^{2}(x, y)\right]+\Sigma_{t, g}(x, y) \phi_{g}^{2}(x, y)= \\
\frac{2}{5}\left\{\Sigma_{r, g}(x, y) \phi_{g}^{0}(x, y)-\left(\frac{\chi_{g}(x, y)}{k_{e f f}} \sum_{g^{\prime}} \nu \Sigma_{f, g^{\prime}}(x, y) \phi_{g^{\prime}}^{0}(x, y)+\sum_{g^{\prime} \neq g} \Sigma_{s, g^{\prime} \rightarrow g}(x, y) \phi_{g^{\prime}}^{0}(x, y)\right)\right\}
\end{gathered}
$$

These equations can be rewritten in a heterogeneous form as:

$$
\begin{gathered}
\frac{\partial}{\partial x}\left(-D_{g}(x, y) \frac{\partial}{\partial x} \Phi_{g}(x, y)\right)+\frac{\partial}{\partial y}\left(-D_{g}(x, y) \frac{\partial}{\partial y} \Phi_{g}(x, y)\right)+\Sigma_{r, g}(x, y) \Phi_{g}(x, y)= \\
\frac{\chi_{g}(x, y)}{k_{e f f}} \sum_{g^{\prime}} \nu \Sigma_{f, g^{\prime}}(x, y) \phi_{g^{\prime}}^{0}(x, y)+\sum_{g^{\prime} \neq g} \Sigma_{s, g^{\prime} \rightarrow g}(x, y) \phi_{g^{\prime}}^{0}(x, y)+2 \Sigma_{r, g}(x, y) \phi_{g}^{2}(x, y)
\end{gathered}
$$

$$
\begin{gathered}
\frac{27}{35}\left[\frac{\partial}{\partial x}\left(-D_{g}(x, y) \frac{\partial}{\partial x} \phi_{g}^{2}(x, y)\right)+\frac{\partial}{\partial y}\left(-D_{g}(x, y) \frac{\partial}{\partial y} \phi_{g}^{2}(x, y)\right)\right]+\Sigma_{t, g}(x, y) \phi_{g}^{2}(x, y)= \\
\frac{2}{5}\left\{\Sigma_{r, g}(x, y) \phi_{g}^{0}(x, y)-\left(\frac{\chi_{g}(x, y)}{k_{e f f}} \sum_{g^{\prime}} \nu \Sigma_{f, g^{\prime}}(x, y) \phi_{g^{\prime}}^{0}(x, y)+\sum_{g^{\prime} \neq g} \Sigma_{s, g^{\prime} \rightarrow g}(x, y) \phi_{g^{\prime}}^{0}(x, y)\right)\right\}
\end{gathered}
$$

And using the Fick's law, eq.5.82 is converted into: 


$$
\begin{gathered}
\frac{\partial}{\partial x} J_{g}^{0, x}+\frac{\partial}{\partial y} J_{g}^{0, y}+\Sigma_{r, g}(x, y) \Phi_{g}(x, y)= \\
\frac{\chi_{g}(x, y)}{k_{e f f}} \sum_{g^{\prime}} \nu \Sigma_{f, g^{\prime}}(x, y) \phi_{g^{\prime}}^{0}(x, y)+\sum_{g^{\prime} \neq g} \Sigma_{s, g^{\prime} \rightarrow g}(x, y) \phi_{g^{\prime}}^{0}(x, y)+2 \Sigma_{r, g}(x, y) \phi_{g}^{2}(x, y)
\end{gathered}
$$

Applying the finite difference method to the eq. 5.84 one can obtain:

$$
\begin{aligned}
\frac{J_{i, j, g}^{0, x}}{h_{i}}-\frac{J_{i-1, j, g}^{0, x}}{h_{i}}+\frac{J_{i, j, g}^{0, y}}{k_{j}}-\frac{J_{i, j-1, g}^{0, y}}{k_{j}}+\Sigma_{r, i, j, g} \Phi_{i, j, g}= \\
\quad \frac{\chi_{i, j, g}}{k_{e f f}} \sum_{g^{\prime}} \nu \Sigma_{f, i, j, g^{\prime}} \phi_{i, j, g^{\prime}}^{0}+\sum_{g^{\prime} \neq g} \Sigma_{s, i, j, g^{\prime} \rightarrow g} \phi_{i, j, g^{\prime}}^{0}+2 \Sigma_{r, i, j, g} \phi_{i, j, g}^{2}
\end{aligned}
$$

\section{Where:}

$\frac{J_{i, j, g}^{0, x}}{h_{i}}=-\widetilde{D}_{i, j, g}^{0, x+}\left(\Phi_{i+1, j, g}-\Phi_{i, j, g}\right)=-\widetilde{D}_{i, j, g}^{0, x+}\left(\phi_{i+1, j, g}^{0}+2 \phi_{i+1, j, g}^{2}-\phi_{i, j, g}^{0}-2 \phi_{i, j, g}^{2}\right)$

$$
\frac{J_{i-1, j, g}^{0, x}}{h_{i}}=-\widetilde{D}_{i, j, g}^{0, x-}\left(\Phi_{i, j, g}-\Phi_{i-1, j, g}\right)=-\widetilde{D}_{i, j, g}^{0, x-}\left(\phi_{i, j, g}^{0}+2 \phi_{i, j, g}^{2}-\phi_{i-1, j, g}^{0}-2 \phi_{i-1, j, g}^{2}\right)
$$

$$
\frac{J_{i, j, g}^{0, y}}{k_{j}}=-\widetilde{D}_{i, j, g}^{0, y+}\left(\Phi_{i, j+1, g}-\Phi_{i, j, g}\right)=-\widetilde{D}_{i, j, g}^{0, y+}\left(\phi_{i, j+1, g}^{0}+2 \phi_{i, j+1, g}^{2}-\phi_{i, j, g}^{0}-2 \phi_{i, j, g}^{2}\right)
$$

$$
\frac{J_{i, j-1, g}^{0, y}}{k_{j}}=-\widetilde{D}_{i, j, g}^{0, y-}\left(\Phi_{i, j, g}-\Phi_{i, j-1, g}\right)=-\widetilde{D}_{i, j, g}^{0, y-}\left(\phi_{i, j, g}^{0}+2 \phi_{i, j, g}^{2}-\phi_{i, j-1, g}^{0}-2 \phi_{i, j-1, g}^{2}\right)
$$


and eq.5.83 is transformed into:

$$
\begin{aligned}
& \frac{27}{35}\left[\frac{\partial}{\partial x} J_{g}^{2, x}+\frac{\partial}{\partial y} J_{g}^{2, y}\right]+\Sigma_{t, g}(x, y) \phi_{g}^{2}(x, y)= \\
& \frac{2}{5}\left\{\Sigma_{r, g}(x, y) \phi_{g}^{0}(x, y)-\left(\frac{\chi_{g}(x, y)}{k_{e f f}} \sum_{g^{\prime}} \nu \Sigma_{f, g^{\prime}}(x, y) \phi_{g^{\prime}}^{0}(x, y)+\sum_{g^{\prime} \neq g} \Sigma_{s, g^{\prime} \rightarrow g}(x, y) \phi_{g^{\prime}}^{0}(x, y)\right)\right\}
\end{aligned}
$$

Applying th finite difference method to the eq.5.90 it can be obtained:

$$
\begin{aligned}
& \frac{J_{i, j, g}^{2, x}}{h_{i}}-\frac{J_{i-1, j, g}^{2, x}}{h_{i}}+\frac{J_{i, j, g}^{2, y}}{k_{j}}-\frac{J_{i, j-1, g}^{2, y}}{k_{j}}+\Sigma_{t, i, j, g} \phi_{i, j, g}^{2}= \\
& \frac{2}{5}\left\{\Sigma_{r, i, j, g} \phi_{i, j, g}^{0}-\left(\frac{\chi_{i, j, g}}{k_{e f f}} \sum_{g^{\prime}} \nu \Sigma_{f, i, j, g^{\prime}} \phi_{i, j, g^{\prime}}^{0}+\sum_{g^{\prime} \neq g} \Sigma_{s, i, j, g^{\prime} \rightarrow g} \phi_{i, j, g^{\prime}}^{0}\right)\right\}
\end{aligned}
$$

Where:

$$
\begin{gathered}
\frac{J_{i, j, g}^{2, x}}{h_{i}}=-\widetilde{D}_{i, j, g}^{2, x+}\left(\phi_{i+1, j, g}^{2}-\phi_{i, j, g}^{2}\right) \\
\frac{J_{i-1, j, g}^{2, x}}{h_{i}}=-\widetilde{D}_{i, j, g}^{2, x-}\left(\phi_{i, j, g}^{2}-\phi_{i-1, j, g}^{2}\right) \\
\frac{J_{i, j, g}^{2, y}}{k_{j}}=-\widetilde{D}_{i, j, g}^{2, y+}\left(\phi_{i, j+1, g}^{2}-\phi_{i, j, g}^{2}\right) \\
\frac{J_{i, j-1, g}^{2, y}}{k_{j}}=-\widetilde{D}_{i, j, g}^{2, y-}\left(\phi_{i, j, g}^{2}-\phi_{i, j-1, g}^{2}\right)
\end{gathered}
$$

By means of the same procedure used for the one-dimensional equations, the discretized two dimensional equations can be written as: 


$$
\begin{gathered}
-\widetilde{D}_{i, j, g}^{0, x-} \Phi_{i-1, j, g}-\widetilde{D}_{i, j, g}^{0, y-} \Phi_{i, j-1, g}-\widetilde{D}_{i, j, g}^{0, x+} \Phi_{i+1, j, g}-\widetilde{D}_{i, j, g}^{0, y+} \Phi_{i, j+1, g}+ \\
{\left[\widetilde{D}_{i, j, g}^{0, x-}+\widetilde{D}_{i, j, g}^{0, x+}+\widetilde{D}_{i, j, g}^{0, y-}+\widetilde{D}_{i, j, g}^{0, y+}+\Sigma_{r, i, j, g}\right] \Phi_{i, j, g}=} \\
\frac{\chi_{i, j, g}}{k_{e f f}} \sum_{g^{\prime}} \nu \Sigma_{f, i, j, g^{\prime}} \phi_{i, j, g^{\prime}}^{0}+\sum_{g^{\prime} \neq g} \Sigma_{s, i, j, g^{\prime} \rightarrow g} \phi_{i, j, g^{\prime}}^{0}+2 \Sigma_{r, i, j, g} \phi_{i, j, g}^{2} \\
-\widetilde{D}_{i, j, g}^{2, x-} \phi_{i-1, j, g}^{2}-\widetilde{D}_{i, j, g}^{2, y-} \phi_{i, j-1, g}^{2}-\widetilde{D}_{i, j, g}^{2, x+} \phi_{i+1, j, g}^{2}-\widetilde{D}_{i, j, g}^{2, y+} \phi_{i, j+1, g}^{2}+ \\
{\left[\widetilde{D}_{i, j, g}^{2, x-}+\widetilde{D}_{i, j, g}^{2, x+}+\widetilde{D}_{i, j, g}^{2, y-}+\widetilde{D}_{i, j, g}^{2, y+}+\Sigma_{t, i, j, g}\right] \phi_{i, j, g}^{2}=} \\
\frac{2}{5} \Sigma_{r, i, j, g} \phi_{i, j, g}^{0}-\frac{2}{5} \frac{\chi_{i, j, g}}{k_{e f f}} \sum_{g^{\prime}} \nu \Sigma_{f, i, j, g^{\prime}} \phi_{i, j, g^{\prime}}^{0}-\frac{2}{5} \sum_{g^{\prime} \neq g} \Sigma_{s, i, j, g^{\prime} \rightarrow g} \phi_{i, j, g^{\prime}}^{0}
\end{gathered}
$$

where:

$$
\begin{gathered}
\widetilde{D}_{i, j, g}^{m, x+}=\frac{2 D_{i, j, g}^{m} D_{i+1, j, g}^{m}}{\left(D_{i, j, g}^{m} h_{i+1}+D_{i+1, j, g}^{m} h_{i}\right) h_{i}} \\
\widetilde{D}_{i, j, g}^{m, x-}=\frac{2 D_{i-1, j, g}^{m} D_{i, j, g}^{m}}{\left(D_{i-1, j, g}^{m} h_{i}+D_{i, j, g}^{m} h_{i-1}\right) h_{i}} \\
\widetilde{D}_{i, j, g}^{m, y+}=\frac{2 D_{i, j, g}^{m} D_{i, j+1, g}^{m}}{\left(D_{i, j, g}^{m} k_{j+1}+D_{i, j+1, g}^{m} k_{j}\right) k_{j}} \\
\widetilde{D}_{i, j, g}^{m, y-}=\frac{2 D_{i, j-1, g}^{m} D_{i, j, g}^{m}}{\left(D_{i, j-1, g}^{m} k_{j}+D_{i, j, g}^{m} k_{j-1}\right) k_{j}} \\
\Phi_{i, j, g}=\phi_{i, j, g}^{0}+2 \phi_{i, j, g}^{2} \\
D_{i, j, g}^{0}=D_{i, j, g}=\frac{1}{3 \Sigma_{t, i, j, g}} \\
D_{i, j, g}^{2}=\frac{27}{35} D_{i, j, g}
\end{gathered}
$$


One can derive the three-dimensional formulation from the formulation stated in this section. This formulation can be easily extrapolated to three-dimensional problems. Three-dimensional formulations have not been presented in this work for brevity. The numerical results in the next section include solutions of several multidimensional problems.

The author wants to highlight that the presentation of the discretized vacuum boundary conditions have not been presented in other works. Prior to Brantley the correct expression might not have been known. Given the complexity of the vacuum boundary conditions it is possible early methods were using approximate vacuum boundary conditions.

\subsection{Eigenvalue problem}

Since the $\mathrm{SP}_{3}$ method consist of two coupled diffusive equations, most numerical methods applied to solve the diffusion equation can be used to solve the eigenvalue problem obtained with the $S P_{3}$ equations. So, the algorithms explained in this chapter make use of the same solver used in the neutron diffusion chapter by means of PETSc and SLEPc libraries.

After testing several iterative methods implemented in SLEPc library to solve eigenvalues problems, the method selected for solving $S P_{3}$ equations is GMRES and incomplete LU factorization.

Fig.5.6-5.11 show the $A$ matrix shape for different problems with the $S P_{3}$ method. It is easy to relate this matrix form with those obtained in the previous chapter, since the matrices shape is very similar to the matrices defined by the diffusion method. This is one of the advantages of the $S P_{3}$ method, since most of the solvers used for Diffusion can be also used for $S P_{3}$. 


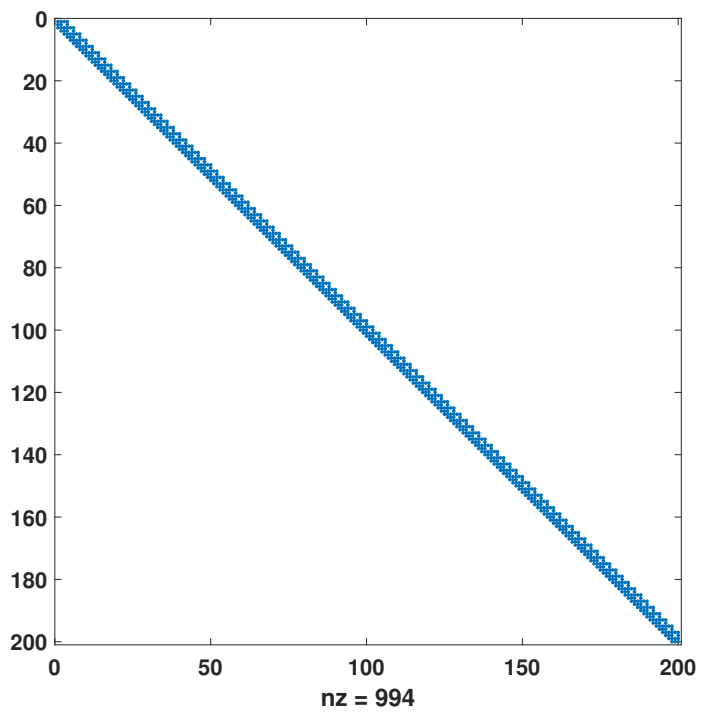

Figure 5.6: Matrix $A, 1 \mathrm{D}-1 \mathrm{EG}-S P_{3}-100$ cells.

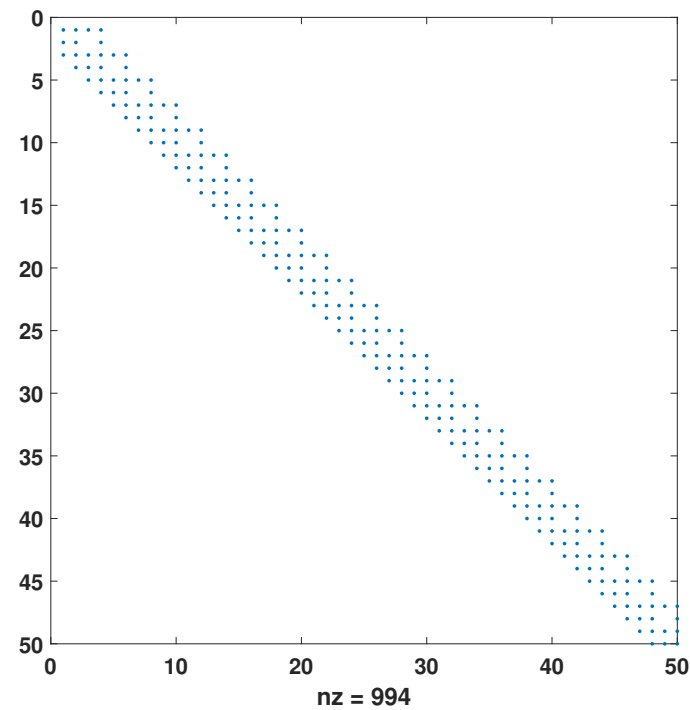

Figure 5.7: Matrix $A, 1 \mathrm{D}-1 \mathrm{EG}-S P_{3}-100$ cells-zoom. 




Figure 5.8: Matrix $A, 1 \mathrm{D}-2 \mathrm{EG}-S P_{3}-100$ cells.

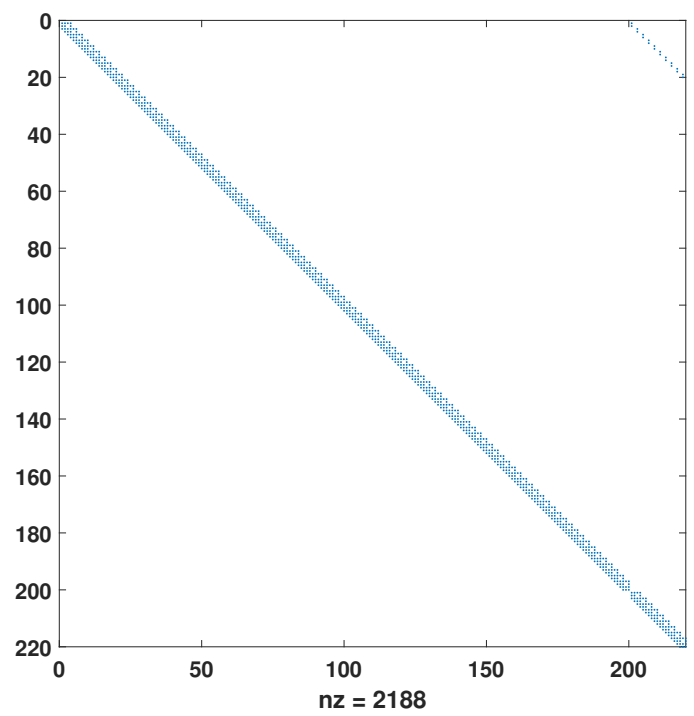

Figure 5.9: Matrix $A, 1 \mathrm{D}-2 \mathrm{EG}-S P_{3}-100$ cells-zoom. 


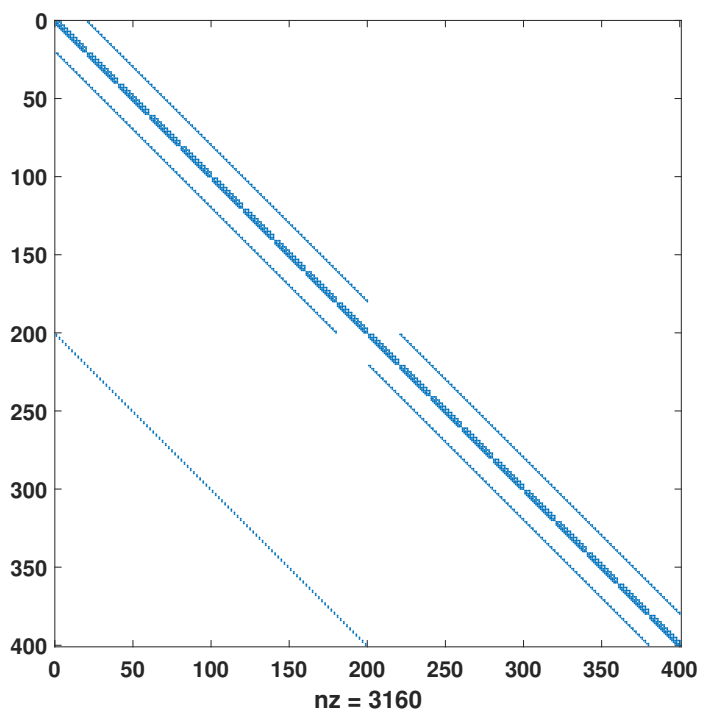

Figure 5.10: Matrix $A, 2 \mathrm{D}-2 \mathrm{EG}-S P_{3}-100$ cells.

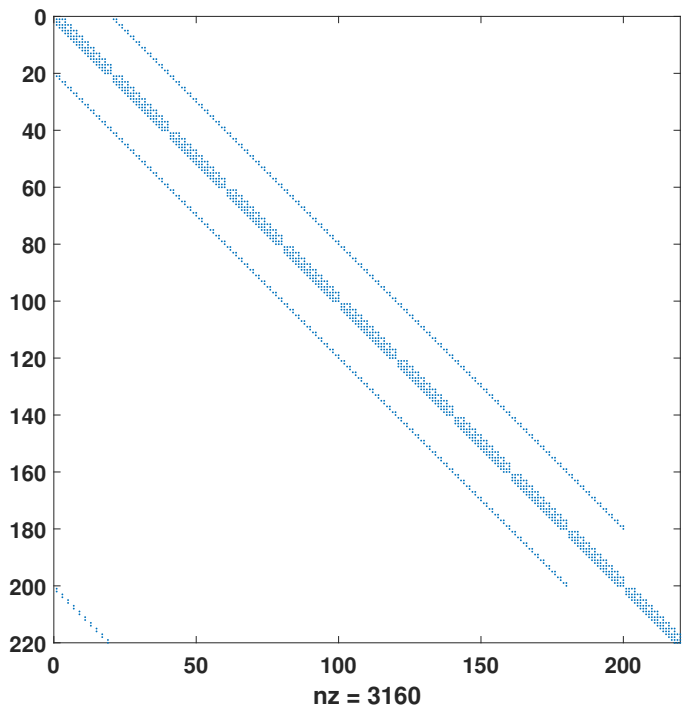

Figure 5.11: Matrix $A, 2 \mathrm{D}-2 \mathrm{EG}-S P_{3}-100$ cells-zoom. 


\subsection{Numerical Results 1D $S P_{3}$ equations}

The numerical methods proposed in the previous section were implemented in the FORTRAN program called SHE3NA (simplified Spherical Harmonics Equations sp3 Neutron Aproximation). This code implements the $S P_{3}$ equations explained above and it was included into ValTran. So, ValTran includes the three neutron transport approaches $S_{N}$, Diffusion and $S P_{3}$. Thus, ValTran can use the same input to calculate results with any of the three approaches. Then, SHE3NA has been compared with other neutron transport approximations exposed and validated in previous chapters.

Different neutron transport codes are used to validate the results of the developed $S_{3}$ code. Next sections make use of some standard codes as reference values, such as the Discrete Ordinates based method code DANTSYS, as well as FEMFUSSION, a finite element $S P_{3}$ method developed by Vidal Ferràndiz [2018]. Furthermore, analytical solutions from Capilla et al. [2008] are shown to compare with the results calculated by the algorithms implemented in this work.

Next sections show several numerical results for 1D and 2D presented above, and two 3D benchmark problems. These 3D cases are: a 3D homogeneous reactor and the FBR Takeda benchmark from Takeda and Ikeda [1991], Capilla et al. [2012]. Several of them show eigenvalues comparison for the $K_{\text {eff }}$ and subcritical modes between the considered neutron transport approximations, as well as, neutron flux comparison.

\subsubsection{Homogeneous slab reactor}

This problem defined in chapter 4 , is a one-group homogeneous slab $2 \mathrm{~cm}$ thick with $\Sigma_{t}=1.0, \nu \Sigma_{f}=0.25$ and $\Sigma_{s}=0.9$. This problem can be found in Capilla et al. [2005] solved with the approaches $P_{1}, P_{3}, P_{5}$ and with code ONEDANT. However, new results were calculated in section 4.6.1. The values calculated with the discrete ordinates codes PARTISN and the code developed by the author, n-DOTEC, using discrete ordinates order $P_{n}-T_{n} S_{96}$ were taken as the reference values. The multiplication factors are shown in tables 5.1 and 5.3 for $P_{1}, P_{3}$, whose values are analytic solutions [Capilla et al., 2005], and for the FDM $S P_{3}$ and diffusion codes developed. All the solutions, FDM $S_{N}$ n-DOTEC, FDM $S P_{3}$ and diffusion code were calculated using a mesh of 2048 cells.

The results of the scalar flux can be seen at fig.5.12. The results were compared with the results obtained with the FDM neutron transport code using $S_{96}$, 
n-DOTEC and with another $S P_{3}$ code called FEMFFUSION which uses the finite element method for the spatial discretization Vidal Ferràndiz [2018]. The curves calculated with the FDM cell-centered scheme $S P_{3}^{C}$ and the edge-centered scheme FDM $S P_{3}^{E}$ coincide.

Table 5.1: Values of the first four eigenvalues for a homogeneous slab $2 \mathrm{~cm}$ thick. (Dif. ${ }^{C}$ is the $S P_{1}$ code using cell-centered scheme). (Dif. ${ }^{E}$ is the $S P_{1}$ code using edge-centered scheme).

\begin{tabular}{lcrrcc}
\hline Eig. & $P_{1}$ Analytic Sol. & FDM Diff. & FDM Diff. ${ }^{E}$ & n-DOTEC $S_{96}$ & PARTISN $S_{96}$ \\
\hline $1^{\text {st }}$ & 0.587489 & 0.587489 & 0.587489 & 0.662933 & 0.662933 \\
$2^{\text {nd }}$ & 0.149135 & 0.149135 & 0.149135 & 0.229686 & - \\
$3^{\text {rd }}$ & 0.058380 & 0.058380 & 0.058380 & 0.126680 & - \\
$4^{t h}$ & 0.029602 & 0.029602 & 0.029602 & 0.085656 & - \\
\hline
\end{tabular}

Table 5.2: Values of the first four eigenvalues for a homogeneous slab $2 \mathrm{~cm}$ thick. $\left(S P_{3}^{C}\right.$ is the $S P_{3}$ code using cell-centered scheme). ( $S P_{3}^{E}$ is the $S P_{3}$ code edge-centered scheme).

\begin{tabular}{lcccc}
\hline Eig. & $S P_{3}$ Analytical Sol. & FDM $S P_{3}^{C}$ & FDM $S P_{3}^{E}$ & FDM $S_{96}$ \\
\hline $1^{\text {st }}$ & 0.652956 & 0.652956 & 0.652956 & 0.662933 \\
$2^{\text {nd }}$ & 0.207745 & 0.207745 & 0.207745 & 0.229686 \\
$3^{\text {rd }}$ & 0.096091 & 0.096092 & 0.096091 & 0.126680 \\
$4^{\text {th }}$ & 0.053122 & 0.053122 & 0.053122 & 0.085656 \\
\hline
\end{tabular}

It can be appreciated that the eigenvalues calculated by the diffusion code and the SP3 code are exactly the same as the analytical solutions. Regarding to the flux comparison, fig. 5.12 shows that the $S P_{3}$ approximation is more accurate than the diffusion solution, and it is not as accurate as the transport solution $S_{96}$ as expected, which is considered the reference value.

\section{Boundary conditions comparison}

This section shows the differences between the three vacuum boundary approaches explained in section 2.2.1, 2.2.2 and 2.2.3, for the one-dimensional homogeneous problem.

As can be seen in Table 5.3, the exact solution is only achieved with the third vacuum boundary condition approach (BC3). The second approach (BC2) differs from exact solution about $1 \mathrm{pcm}$ for the fundamental mode, while the first approach (BC1) differs about $10 \mathrm{pcm}$ in the fundamental mode. All the results were 


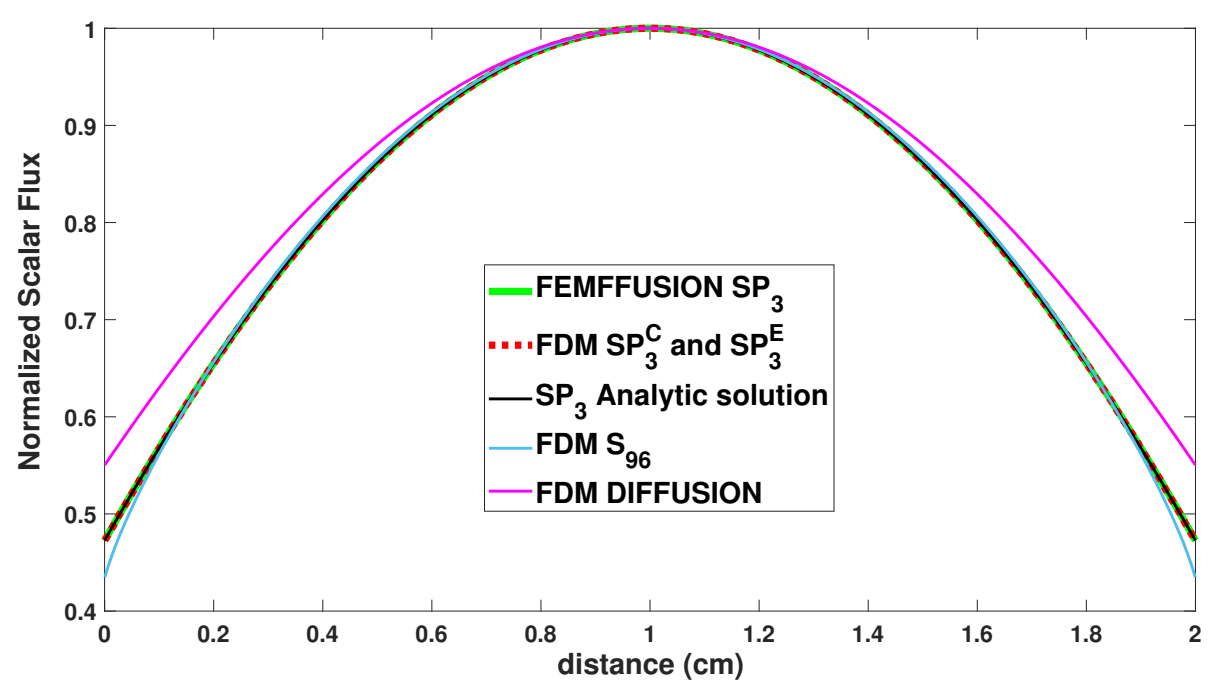

Figure 5.12: Normalized Scalar Flux for Homogeneous 1D reactor.

calculated with the same discretization. Better results can be obtained by using the first and second approaches if the discretization is increased.

Table 5.3: Values of the first four eigenvalues for a homogeneous slab $2 \mathrm{~cm}$ thick. $\left(S P_{3}^{C *}\right.$ is the $S P_{3}$ code using cell-centered scheme and $B C$ refers to each boundary condition approach).

\begin{tabular}{lcccc}
\hline Eig. & $S P_{3}$ Analytical Sol. & FDM $S P_{3}^{C}$ BC3 & FDM $S P_{3}^{C}$ BC2 & FDM $S P_{3}^{C} B C 1$ \\
\hline $1^{\text {st }}$ & 0.652956 & 0.652956 & 0.652890 & 0.652493 \\
$2^{\text {nd }}$ & 0.207745 & 0.207745 & 0.207702 & 0.207621 \\
$3^{\text {rd }}$ & 0.096091 & 0.096092 & 0.096074 & 0.096052 \\
$4^{\text {th }}$ & 0.053122 & 0.053122 & 0.053115 & 0.053107 \\
\hline
\end{tabular}

These results suggest that in 1D evaluations of numerical implementations, it would be difficult to ascertain that the incorrect boundary conditions are truly incorrect without careful intensive evaluation. The differences observed in the eigenvalues could easily be assumed to be the result of a spatial discretization error. This is further observed in Fig.5.13, where again, the solutions with the inexact boundary conditions are very close to the correct result, but are wrong. 

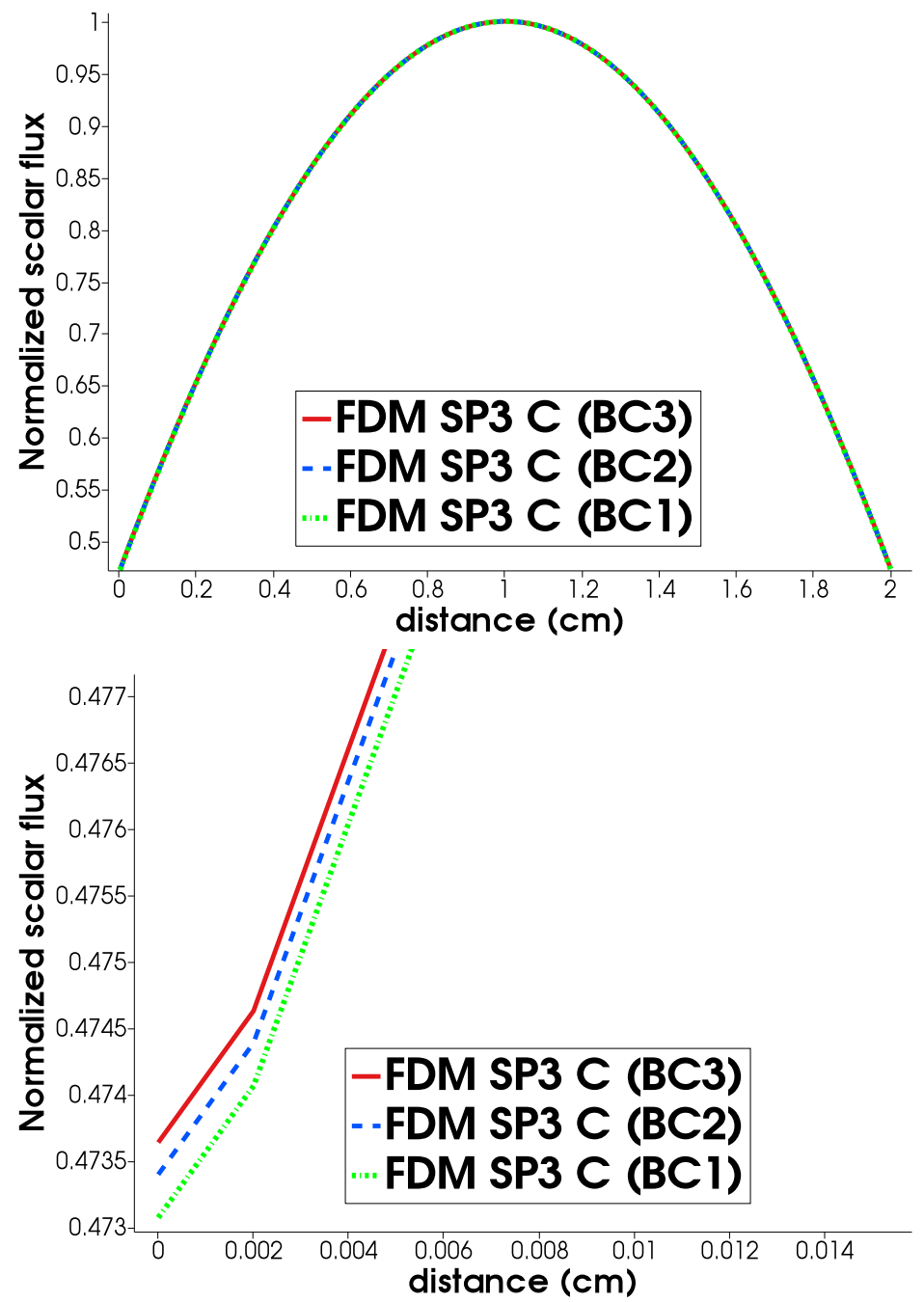

Figure 5.13: Normalized Scalar Flux for Homogeneous 1D reactor and detail of the boundary.

Fig.5.13 shows the normalized scalar flux calculated with the three different boundary condition approaches. In this case, the difference among the normalized scalar fluxes calculated with the three approaches can be considered negligible. 


\subsubsection{ISSA benchmark}

This case was defined in sections 3.8.1 and 4.6.1., it was selected because it combines two different boundary conditions, reflective and vacuum. Fig.5.14 shows the description of the problem.

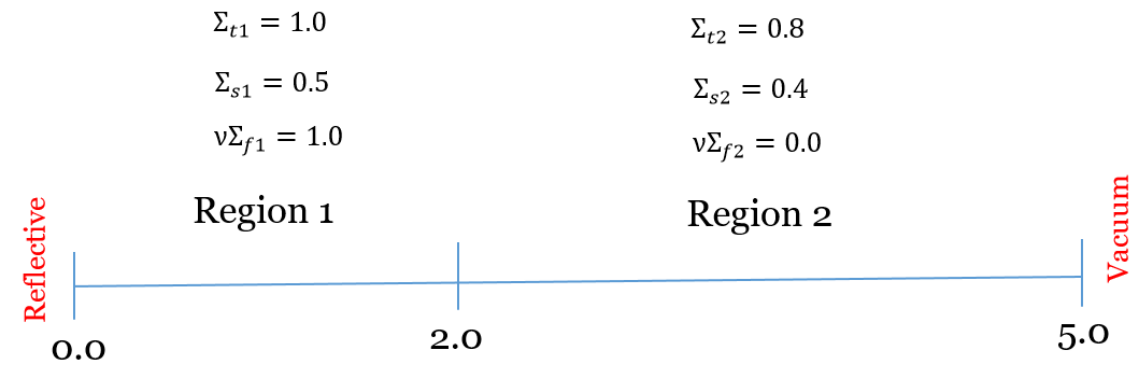

Figure 5.14: Geometry and cross sections for ISSA Benchmark.

To perform a consistent comparison the problem was calculated with the same discretization of section 4.6.1, 2000 cells. From table 5.4 one can conclude that the $S P_{3}$ solutions shows better accuracy than the diffusion calculation. The solution calculated with the $S P_{3}$ method is close to the $S_{96}$ solution, which is shown in fig.5.15-5.18 for the first four modes.

Table 5.4: ISSA problem eigenvalues.

\begin{tabular}{lcccccc}
\hline modes & PARTISN $S_{96}$ & FDM $S_{96}$ n-DOTEC & FDM Diff. ${ }^{C}$ & FDM Diff. ${ }^{E}$ & FDM $S P_{3}^{C *}$ & FDM $S P_{3}^{E *}$ \\
\hline $1^{\text {st }}$ & 1.678794 & 1.678799 & 1.645685 & 1.645684 & 1.675913 & 1.675914 \\
$2^{\text {nd }}$ & - & 0.801363 & 0.604050 & 0.604049 & 0.762315 & 0.762314 \\
$3^{\text {rd }}$ & - & 0.465249 & 0.239767 & 0.239766 & 0.376191 & 0.376191 \\
$4^{\text {th }}$ & - & 0.321525 & 0.120525 & 0.120524 & 0.211399 & 0.211399 \\
\hline
\end{tabular}




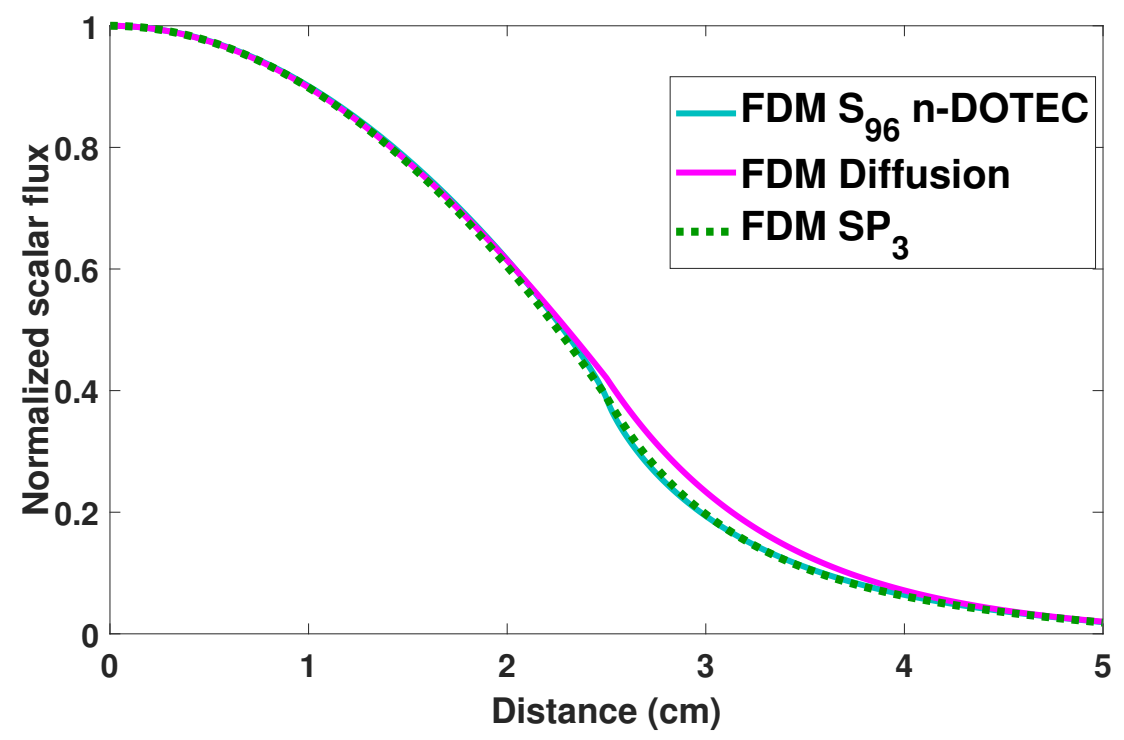

Figure 5.15: ISSA: normalized scalar flux of the first mode.

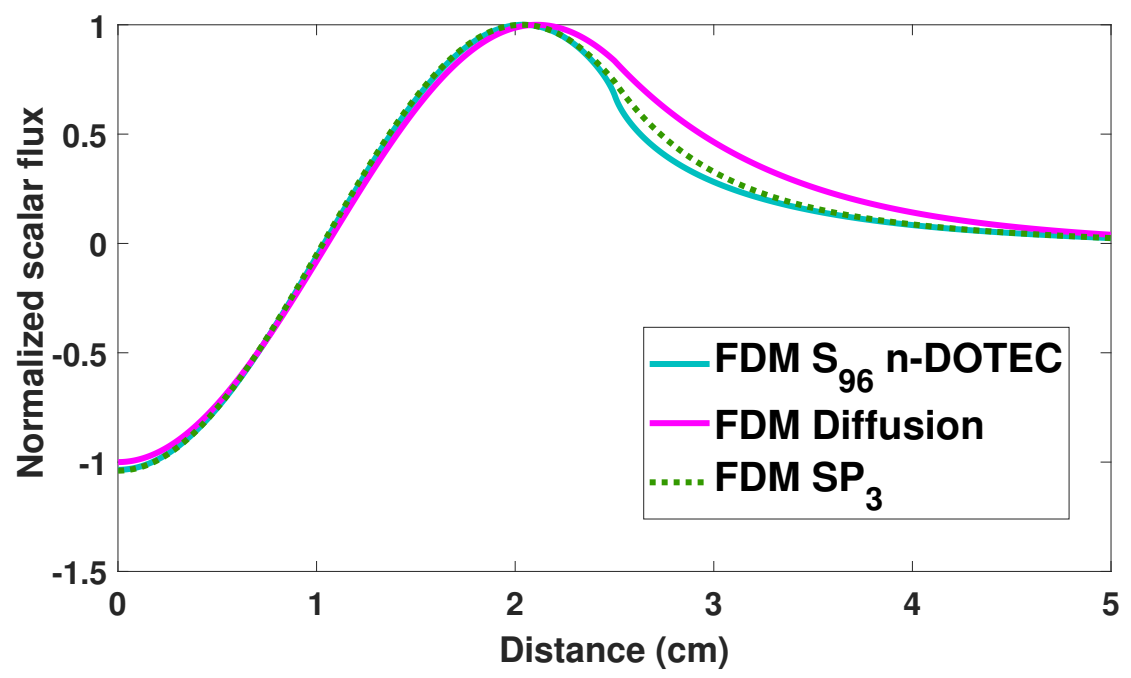

Figure 5.16: ISSA: normalized scalar flux of the second mode. 


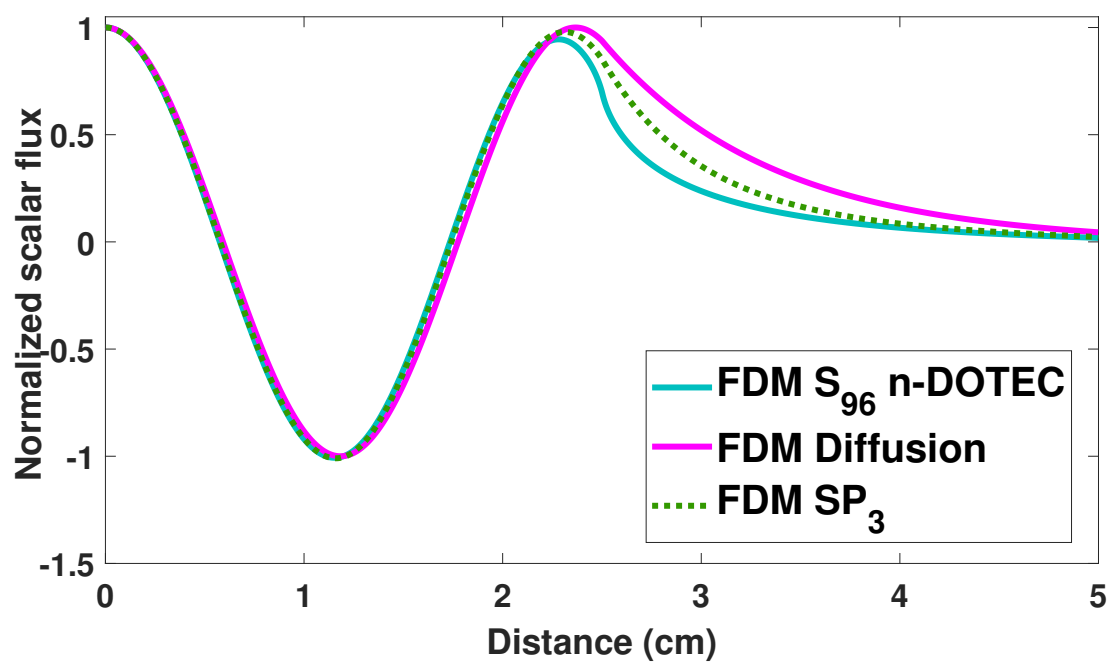

Figure 5.17: ISSAe: normalized scalar flux of the third mode.

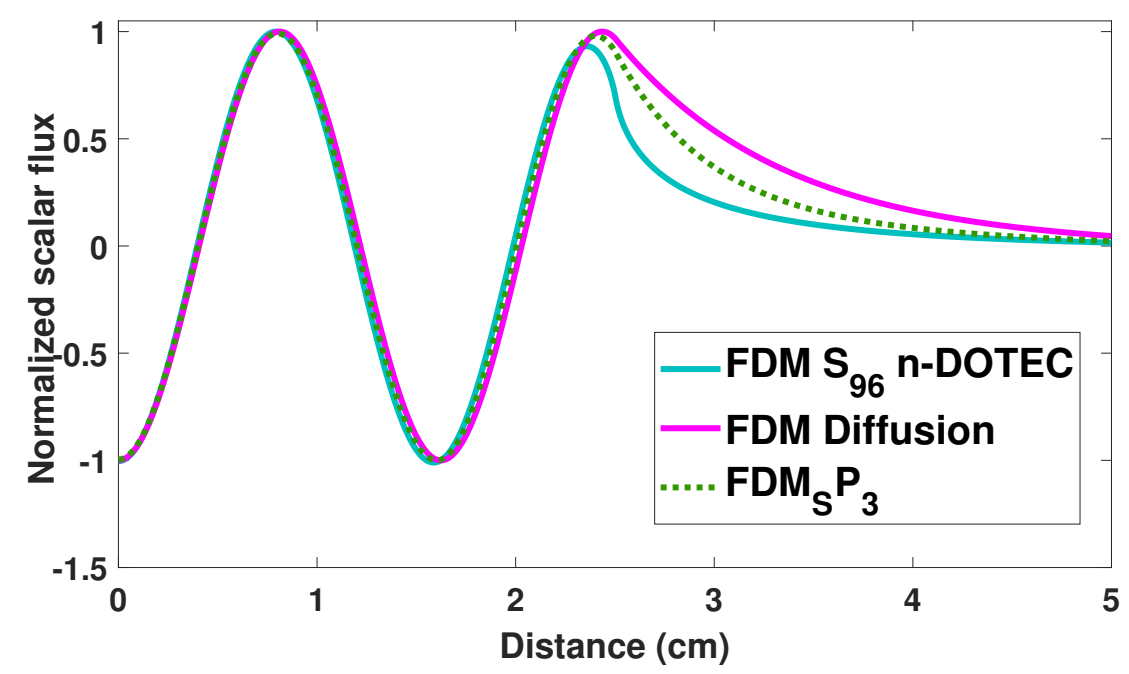

Figure 5.18: ISSA: normalized scalar flux of the fourth mode. 


\subsubsection{Heterogeneous slab test problem}

This problem is described in the previous chapter in section 4.6.1. Table 5.5 show $s$ the cross section of the each material considering one-energy group and a scheme of the problem is shown in fig.5.19. Vacuum conditions are considered for left and right boundaries. The number of mesh cells for each region is 500. A compendium of results is shown in table 5.6, the first column, $P_{3}$, is considered as the reference values to validate the $S P_{3}$ results. The $P_{3}$ values were extracted from Capilla et al. [2005]. However, the reference values of the problem are those obtained by the PARTISN discrete ordinates code with $P_{n}-T_{n} S_{96}$ order. These are the most accurate values but PARTISN is not able to calculate other modes. The FDM $S_{N}$ code developed by the author, which was validated in previous chapters is the reference for the rest of modes. From table 5.6. and figs. 5.20-5.25, one can note that $S P_{3}$ improves significantly the results compared with the diffusion calculations.

Table 5.5: Cross-Sections for heterogeneous slab problem.

\begin{tabular}{lccc}
\hline & $\nu \Sigma_{f}\left(\mathrm{~cm}^{-1}\right)$ & $\Sigma_{s}\left(\mathrm{~cm}^{-1}\right)$ & $\Sigma_{t}\left(\mathrm{~cm}^{-1}\right)$ \\
\hline Fuel (U-235) & 0.178 & 0.334 & 0.416667 \\
Reflector (Be) & 0.0 & 0.334 & 0.37037 \\
Absorber (Be $w / c=0.1)$ & 0.0 & 0.037 & 0.37037 \\
\hline
\end{tabular}

\begin{tabular}{|c|c|c|c|c|c|c|c|}
\hline Base case & $\mathrm{R}$ & Fuel & $R$ & Fuel & $\mathrm{R}$ & Fuel & R \\
\hline Abs. in Pos. 5 & R & Fuel & R & Fuel & Abs. & Fuel & R \\
\hline Abs. in Pos. 6 & R & Fuel & R & Fuel & R & Abs. & R \\
\hline
\end{tabular}

Figure 5.19: Cases for Heterogeneous slab problem.

Table 5.6: Heterogeneous slab problem eigenvalues.

\begin{tabular}{lcccccc}
\hline Case & modes & $P_{3}$ & PARTISN $S_{96}$ & FDM $S_{96}$ n-DOTEC & FDM Diff. & FDM $S P_{3}^{C}$ \\
\hline No absorber & 1st & 1.148740 & 1.162413 & 1.162228 & 1.113872 & 1.148745 \\
& 2nd & 0.735037 & - & 0.752258 & 0.658651 & 0.735037 \\
& 3rd & 0.527647 & - & 0.547565 & 0.423945 & 0.527647 \\
& 4th & - & - & 0.210955 & 0.109256 & 0.165351 \\
\hline Abs. in Pos. 5 & 1st & - & 0.933891 & 0.934122 & 0.846061 & 0.910812 \\
& 2nd & - & - & 0.650383 & 0.533335 & 0.618991 \\
& 3rd & - & - & 0.525017 & 0.401738 & 0.500333 \\
& 4th & - & - & 0.202416 & 0.101340 & 0.155388 \\
\hline Abs. in Pos. 6 & 1st & - & 1.013832 & 1.013802 & 0.943324 & 0.998186 \\
& 2nd & - & - & 0.598874 & 0.480401 & 0.579280 \\
& 3rd & - & - & 0.207386 & 0.105354 & 0.161199 \\
& 4th & - & - & 0.187820 & 0.084083 & 0.139211 \\
\hline
\end{tabular}




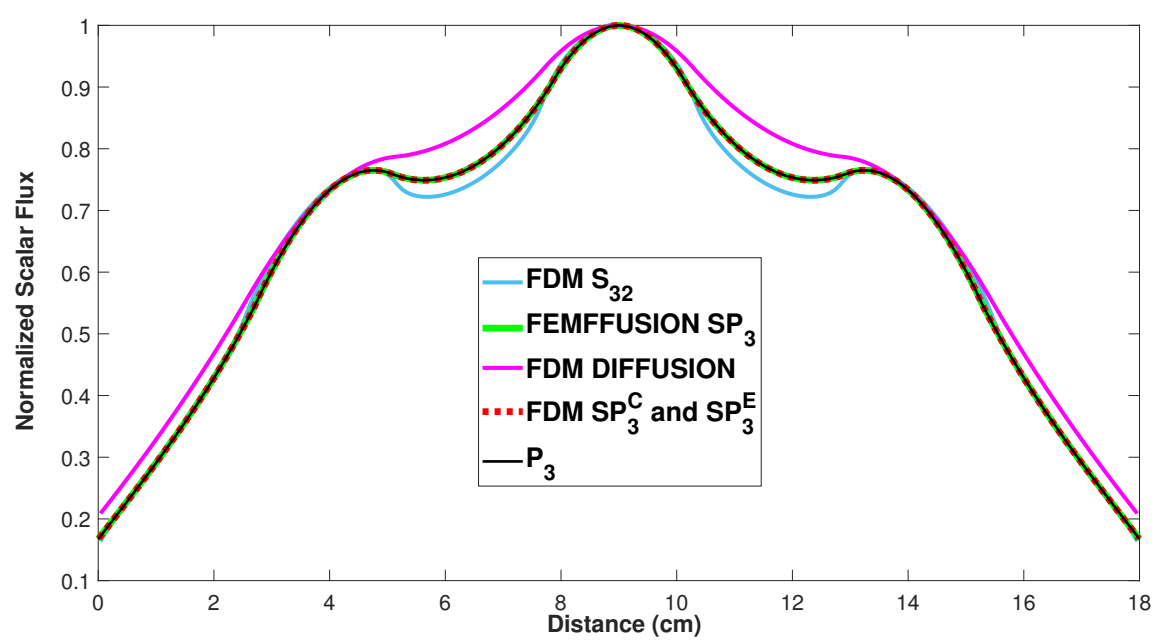

Figure 5.20: Basecase: normalized scalar flux of the first mode.

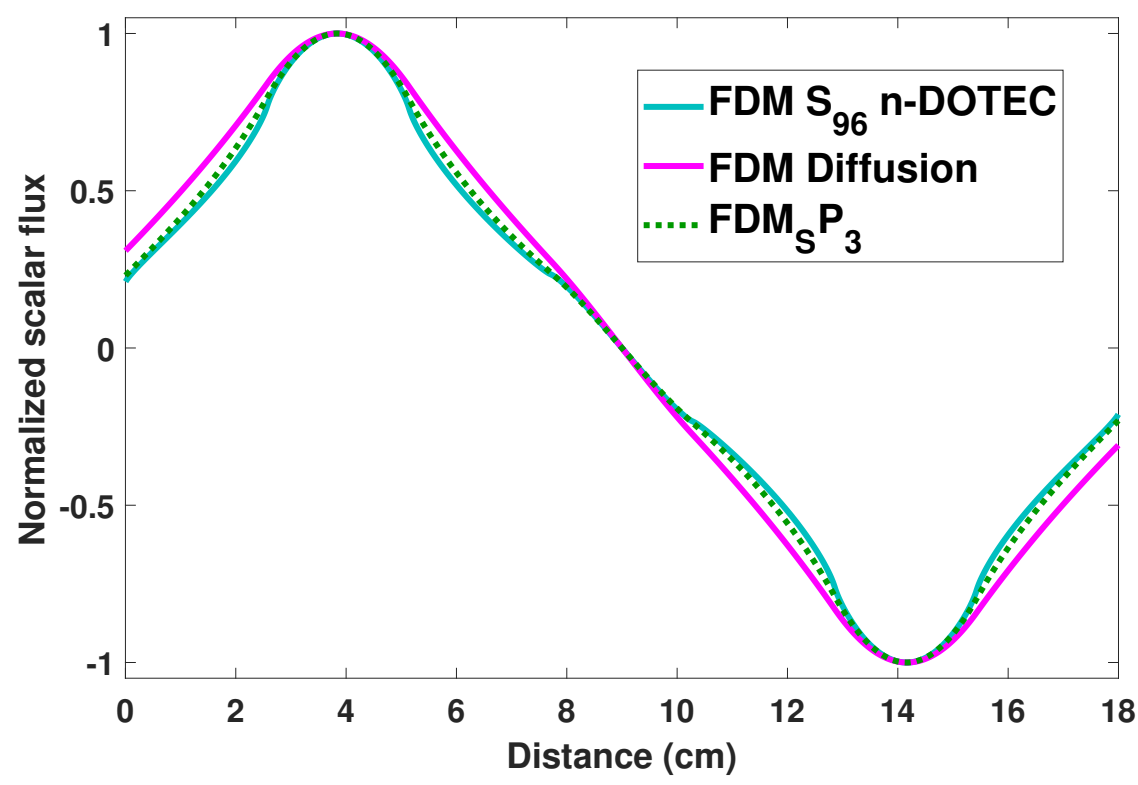

Figure 5.21: Basecase:normalized scalar flux of the second mode. 


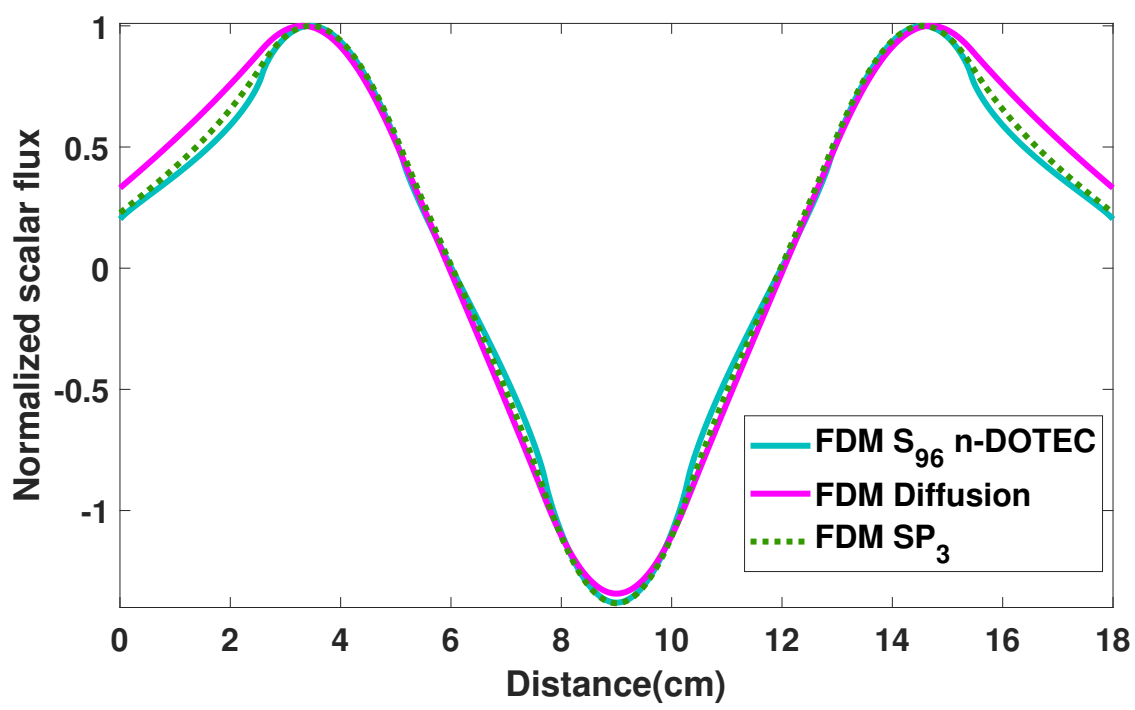

Figure 5.22: Basecase: normalized scalar flux of the third mode.

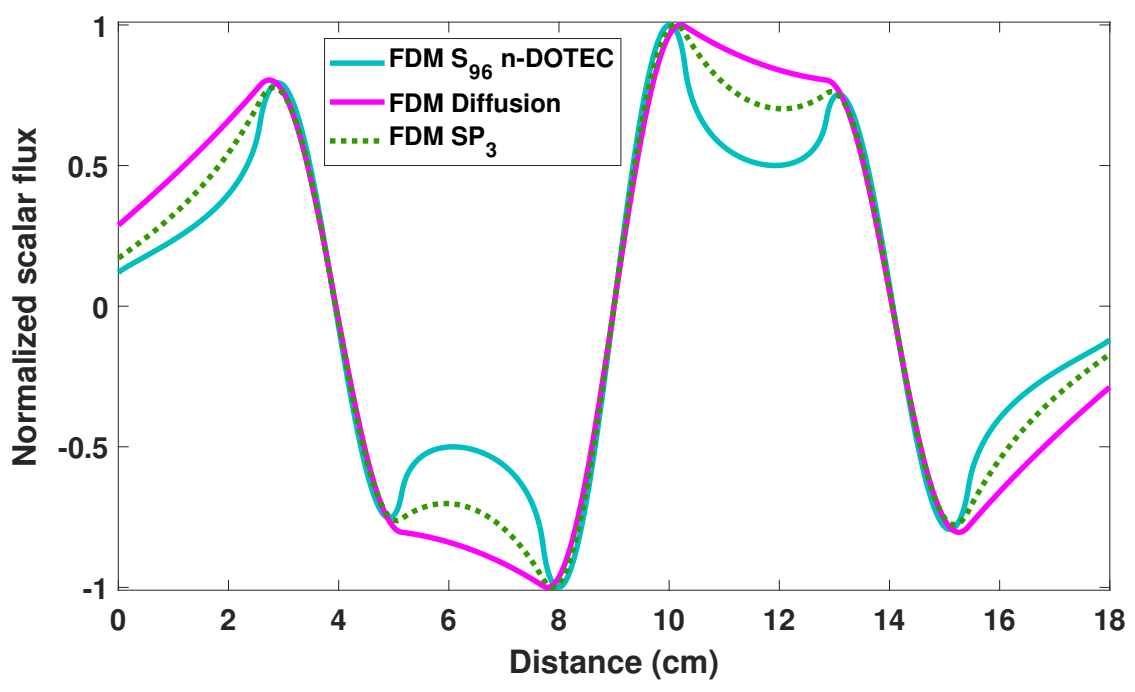

Figure 5.23: Basecase: normalized scalar flux of the fourth mode. 


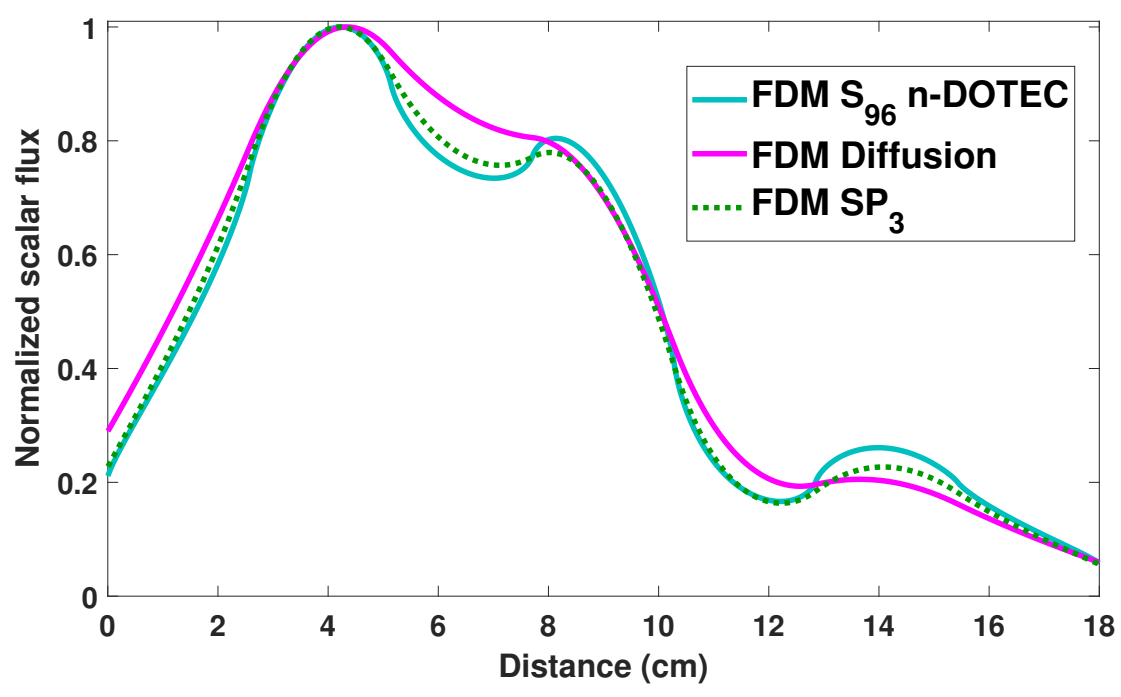

Figure 5.24: Absorber Position 5: normalized scalar flux of the first mode.

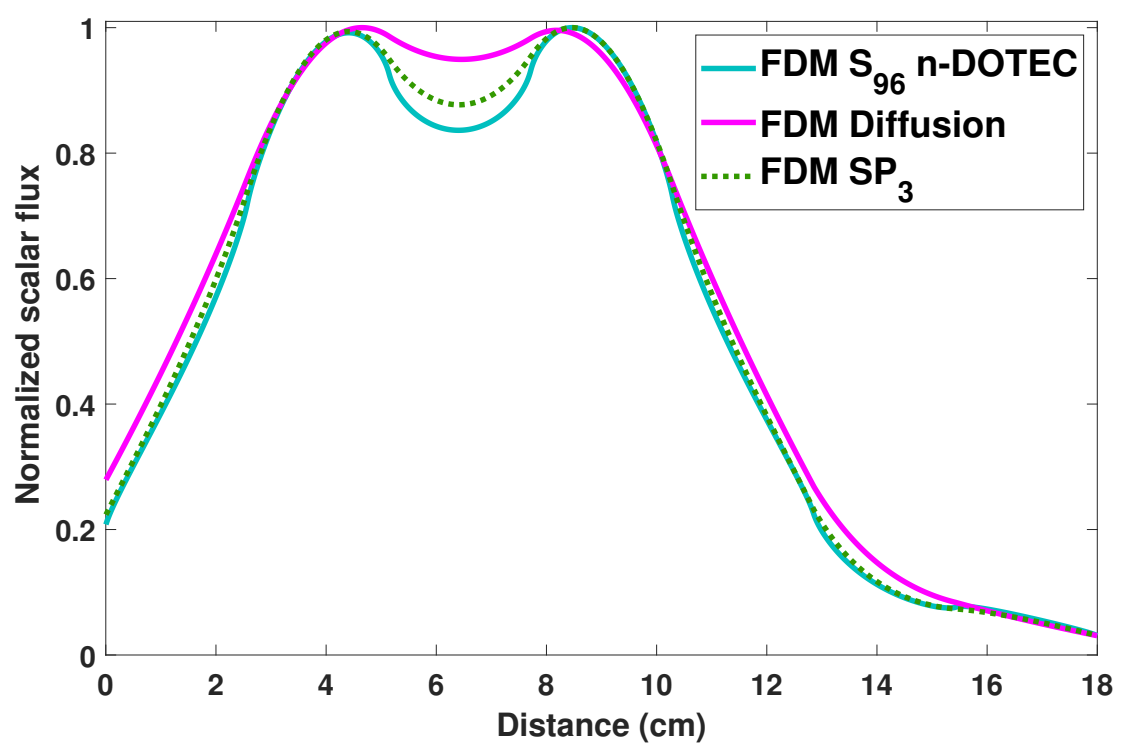

Figure 5.25: Absorber Position 6: normalized scalar flux of the first mode. 


\subsection{Numerical results for 2D $S P_{3}$ equations}

\subsubsection{MOX test problem}

The MOX benchmark problem was described in sections 3.8.2 and 4.6.1. Here, both, SP3 and diffusion calculations were generated with a discretization of 50 cells in $x$ and $y$ axis for each assembly as well as the calculation by using S8 method.

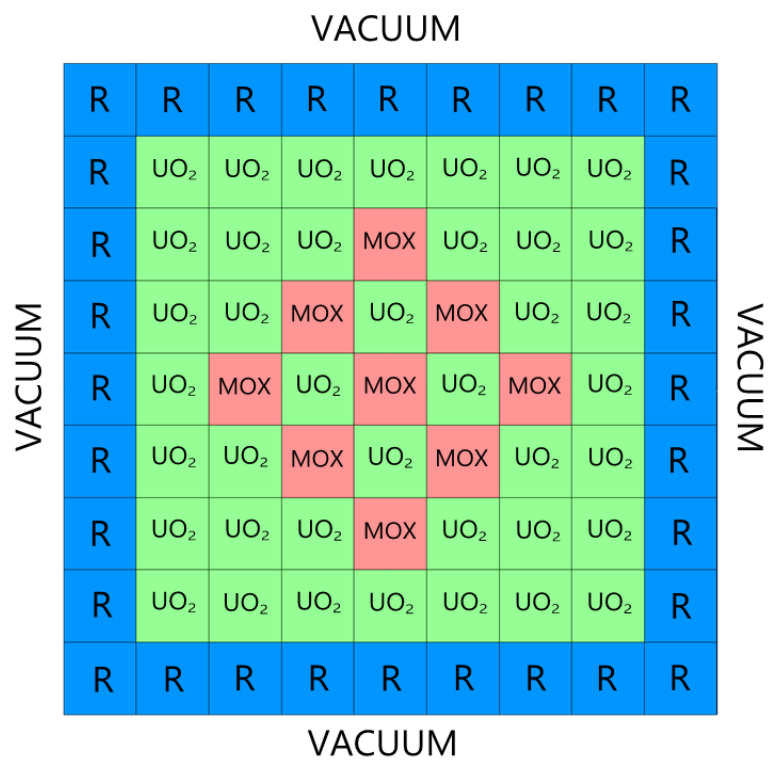

Figure 5.26: MOX benchmark problem geometry.

Four dominant eigenvalues were calculated. The reference solution is calculated by the Spherical Harmonics Nodal Collocation (SHNC) method in Capilla et al. [2008]. The comparison is shown in table 5.8. In figs. 5.27 and 5.28, the neutron scalar flux is shown for the first, second, third and fourth modes. In this case, it can be appreciated that the eigenvalues calculated with the $S P_{3}$ using the cell-centered scheme shows better accuracy than the edge-centered scheme and diffusion approximation. However, this improvement is not seen in the flux distribution. It seems that the problem is highly diffusive and the reflector surrounding fuel makes that flux at the boundary condition do not depend on the methodology used. Moreover the cross sections of the both fuels are similar. With regards to the scalar flux comparison between $S P_{3}$ and $S_{8}$, it is important to highlight that the eigenfluxes corresponding to 2 nd and 3rd mode, which are degenerate due 
to the fact that they have the same eigenvalue, do not have exactly the same distribution. This is a normal condition considering symmetric problems since both eigenfluxes are a linear combination of the solution, and any linear combination could be a solution for this kind of problems although physically it does not make sense. However, it can be seen that the distributions of the 4th eigenflux are exactly the same.

Table 5.7: MOX benchmark problem cross-sections. $g=1$ (fast energy group), $g=2$ (thermal energy group).

\begin{tabular}{ccccccc}
\hline Material & Group & $\Sigma_{t}$ & $\nu \Sigma_{f}$ & $\Sigma_{s, 1 \rightarrow g}$ & $\Sigma_{s, 2 \rightarrow g}$ & $\chi_{g}$ \\
\hline$M O X$ fuel & 1 & 0.550 & 0.0075 & 0.520 & - & 1.000 \\
& 2 & 1.060 & 0.450 & 0.015 & 0.760 & 0.000 \\
$U O_{2}$ fuel & 1 & 0.570 & 0.005 & 0.540 & - & 1.000 \\
& 2 & 1.100 & 0.125 & 0.020 & 1.000 & 0.000 \\
Reflector & 1 & 0.611 & 0.000 & 0.560 & - & 0.000 \\
& 2 & 2.340 & 0.000 & 0.050 & 2.300 & 0.000 \\
\hline
\end{tabular}

Table 5.8: Dominant eigenvalues for the MOX problem. *SHNC=Spherical Harmonics Nodal Collocation.

\begin{tabular}{ccccccc}
\hline Eigenvalue & SHNC $^{*}$ & ${\text { FDM } S P_{3}^{C *}}^{*}$ & FDM Dif. & FDM $S P_{3}^{E *}$ & FDM $D i f .{ }^{E *}$ & FDM $S_{8}$ \\
\hline$k_{\text {eff }}$ & 0.9925 & 0.992505 & 0.992876 & 0.992819 & 0.993133 & 0.992608 \\
2nd eigen. & 0.9665 & 0.966475 & 0.966665 & 0.966721 & 0.966868 & 0.966544 \\
3rd eigen. & 0.9665 & 0.966475 & 0.966665 & 0.966721 & 0.966868 & 0.966544 \\
4th eigen. & 0.9399 & 0.939879 & 0.939807 & 0.940019 & 0.939926 & 0.939900 \\
\hline
\end{tabular}



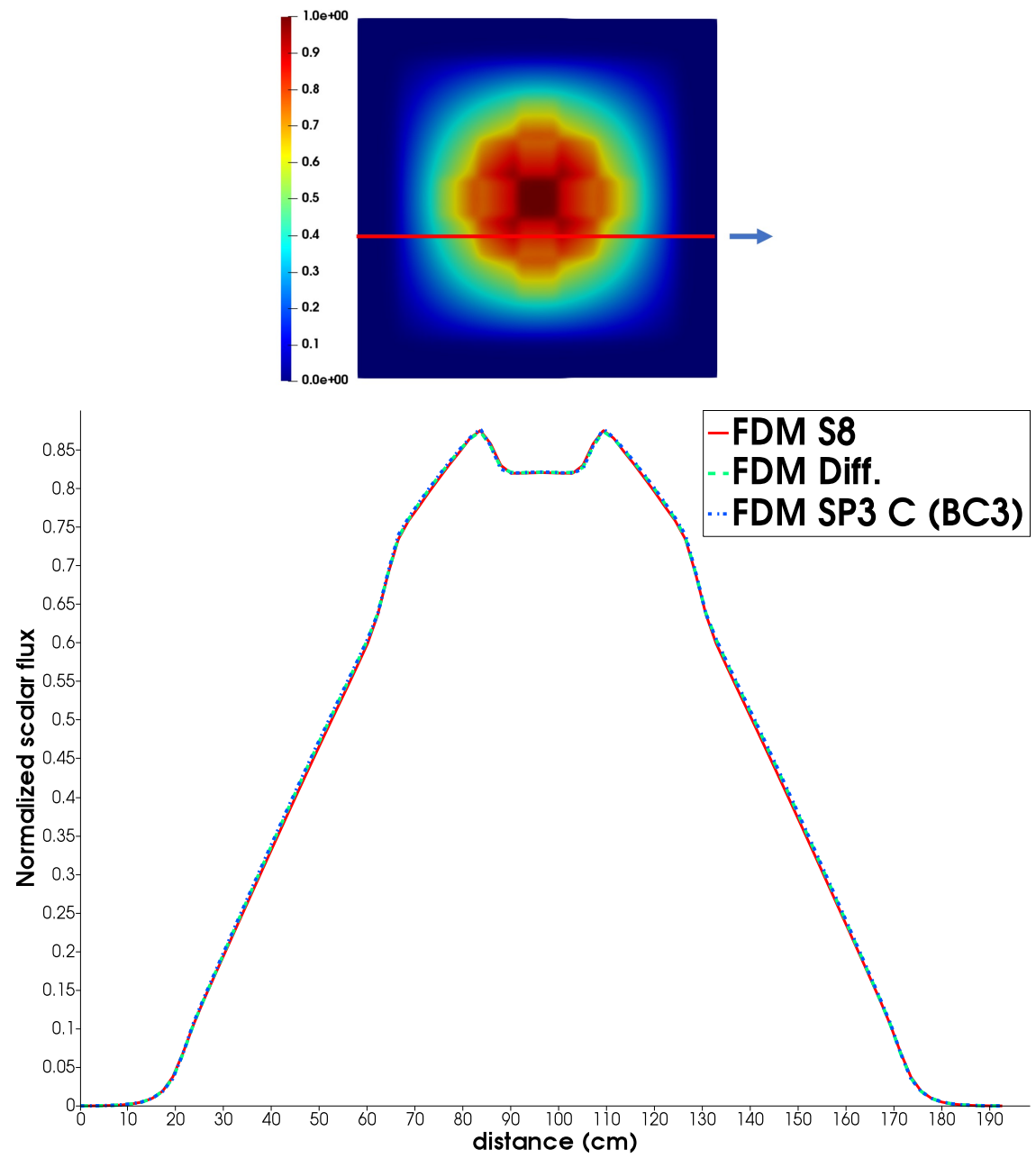

Figure 5.27: MOX: First energy group scalar flux distribution for first mode. 

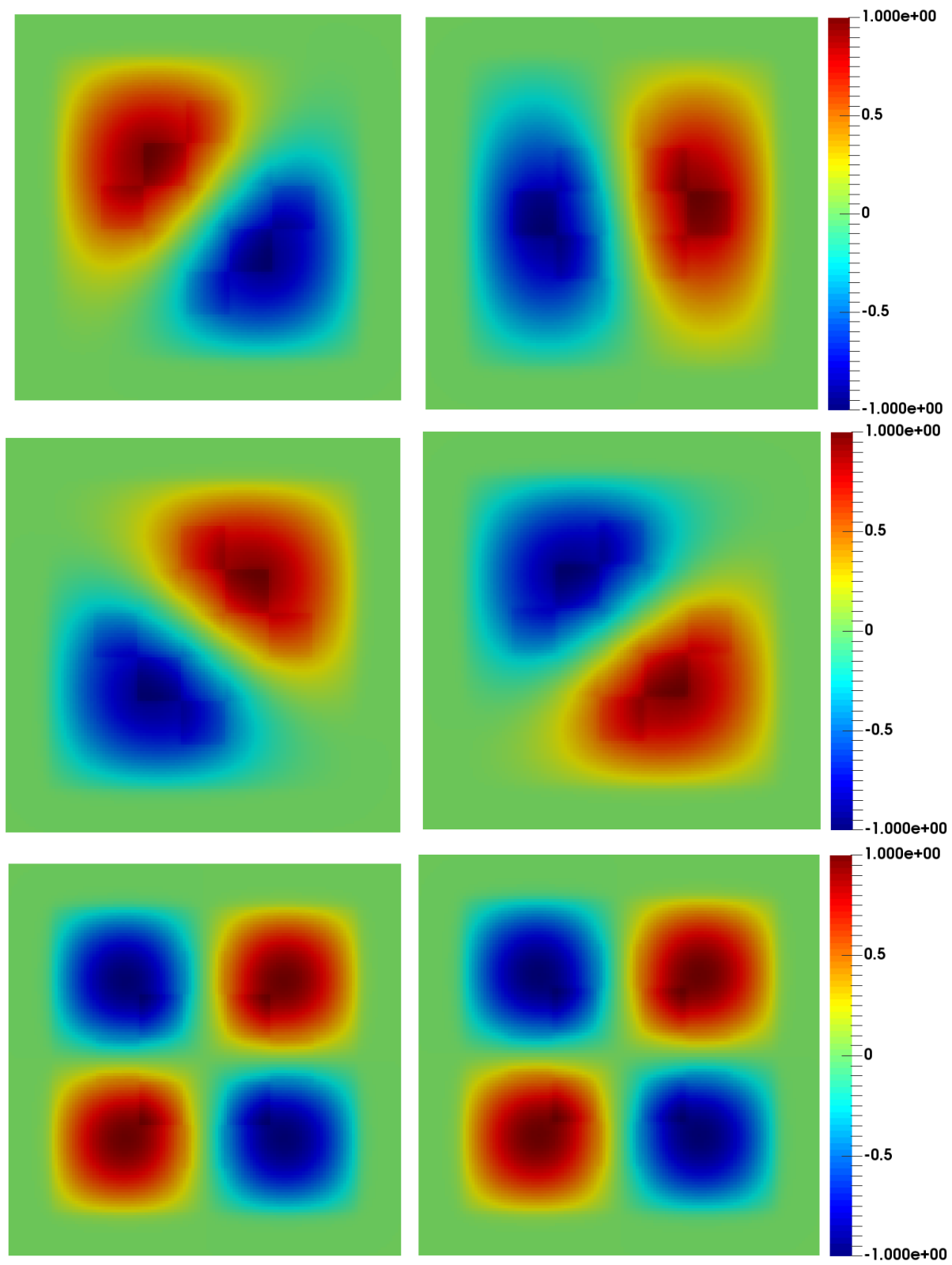

Figure 5.28: MOX: Normalized Scalar flux distribution for $2^{\text {nd }}, 3^{\text {rd }}$ and $4^{\text {th }}$ modes, FDM $S P_{3}^{C}$ on the left, FDM $S_{8}$ on the right. 


\subsubsection{BWR cell test problem}

The following case corresponds to the homogeneous BWR cell presented in sections 3.8.2 and 4.6.1. The reference multiplication factor calculated with DANTSYS (it uses the discrete ordinates method) is 1.212945. FDM $S_{N}, S P_{3}$ and diffusion results were calculated by using a discretization of $30 \times 30$ mesh. Table 5.9 shows the results for the multiplication factor $k_{e f f}$. A flux comparison between $S_{8}, S P_{3}$ and $S P_{1}$ can be seen in Fig.5.30. Fig. 5.31 shows the first group flux neutron distribution for the four dominant eigenvalues. It is easy to see that the cell-centered scheme $S_{3}$ shows better results for the eigenvalue and for the flux distribution than Diffusion or edge-centered $S P_{3}$. Another interesting observation is that the accuracy of the $S P_{3}$ solution seems approximately the same as the $S_{4}$ method. Both differ considerably from the $S_{8}$ solution, but it is helpful to understand intuitively that $S P_{3}$ solutions have approximately the same accuracy as $S_{4}$ solutions, for at least some problems. An important conclusion extracted from this sample is that the shape of the lambda modes is insensitive to the angular approximation.

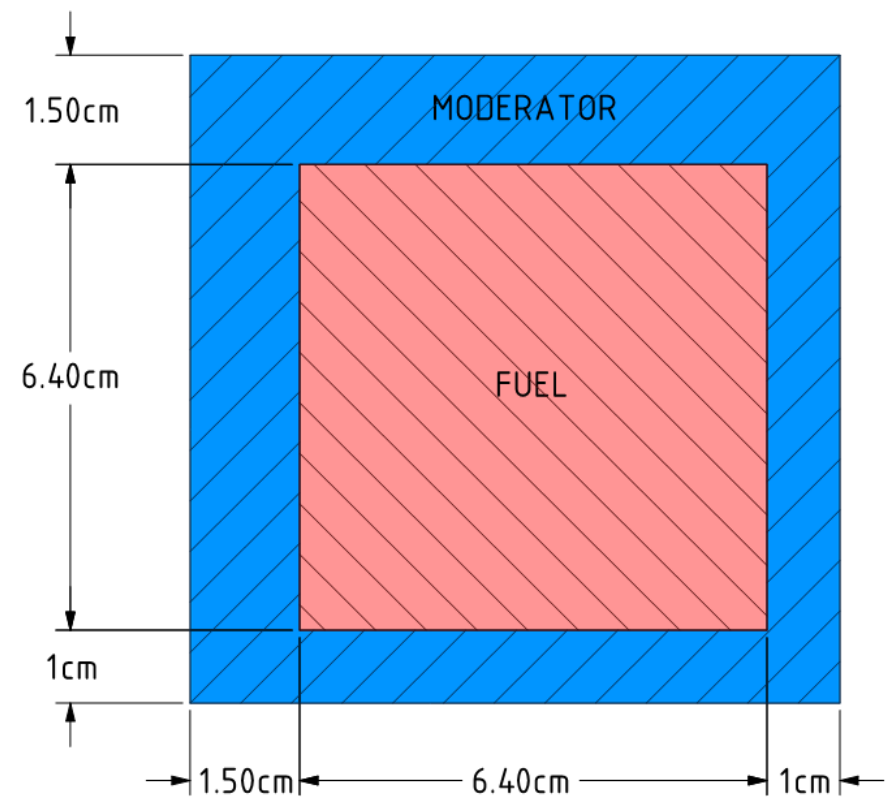

Figure 5.29: BWR cell problem geometry. 
Table 5.9: BWR cell benchmark problem multiplication factor results.

\begin{tabular}{cccc} 
& Order & $K_{\text {eff }}$ & pcm $\left(\Delta K_{\text {eff }}\right)$ \\
\hline DANTSYS & $S_{8}$ & 1.212945 & - \\
FDM $S_{N}$ & $S_{8}$ & 1.212944 & 0 \\
FDM $S P_{3}^{C}$ & - & 1.213237 & 24 \\
FDM $D i f .{ }^{C}$ & - & 1.220100 & 589 \\
FDM $S P_{3}^{E}$ & - & 1.214459 & 124 \\
FDM $D i f .{ }^{E}$ & - & 1.220886 & 654 \\
\hline
\end{tabular}
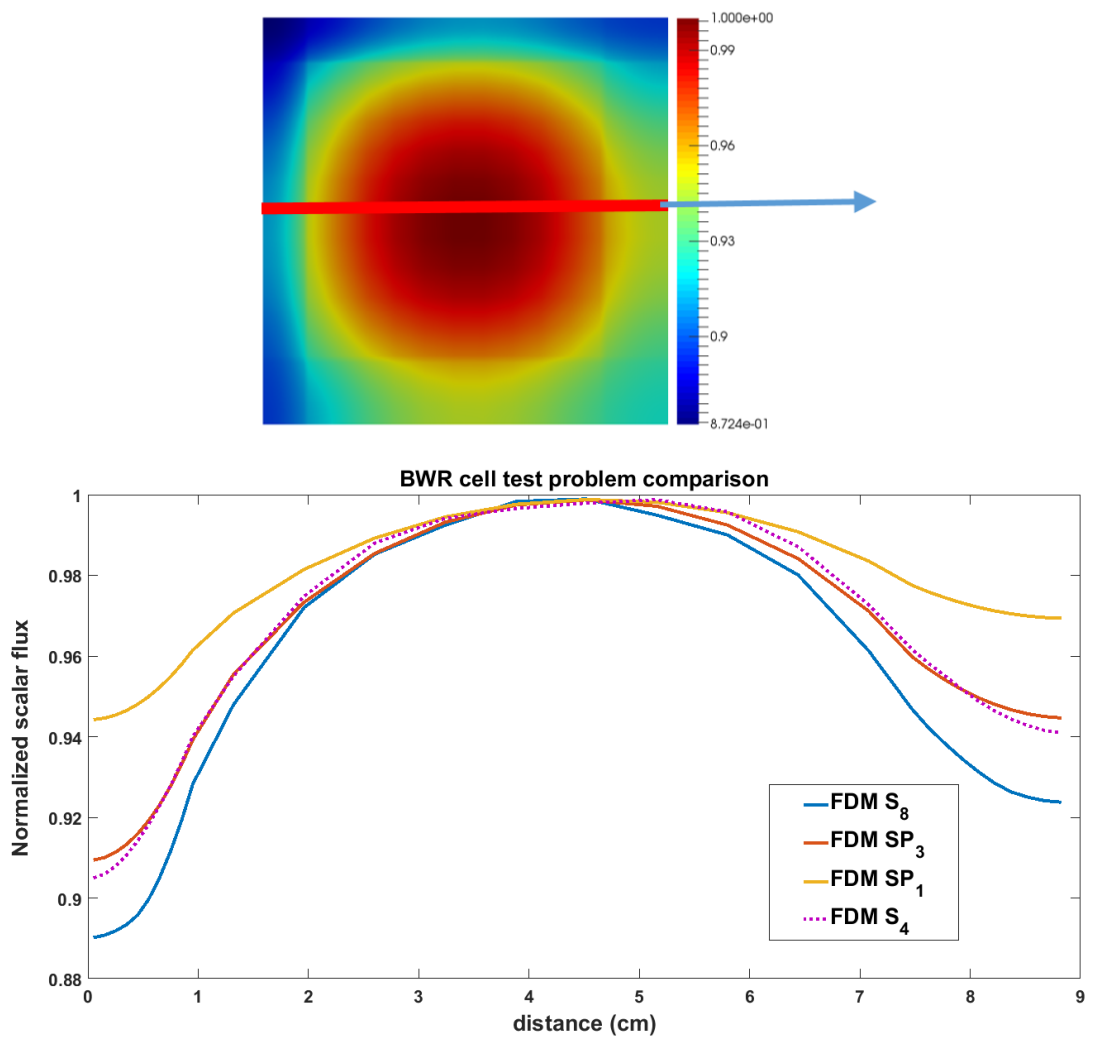

Figure 5.30: BWR cell test: normalized scalar flux distribution for $1^{\text {st }}$ mode, $1^{\text {st }}$ energy group. 

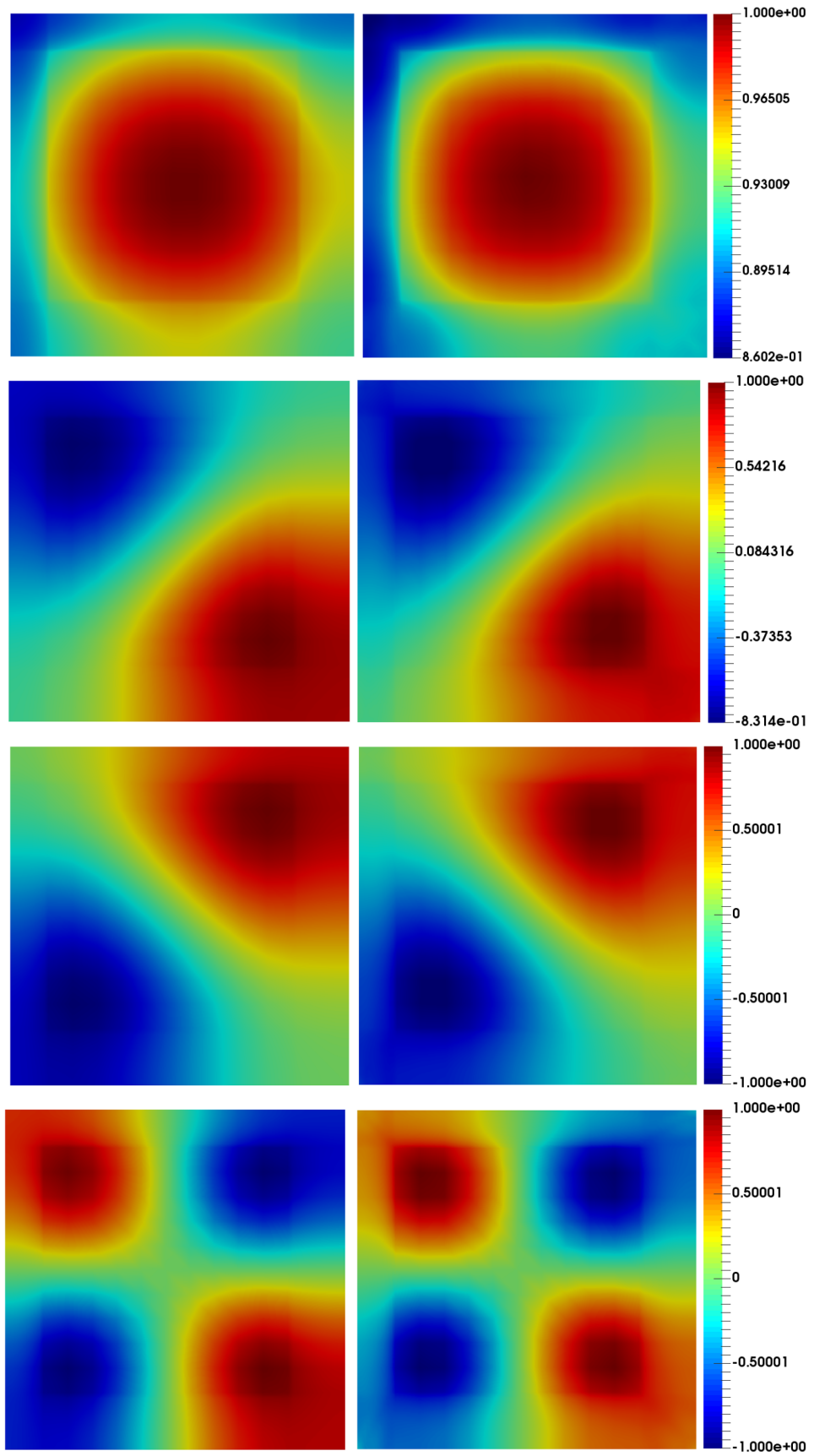

Figure 5.31: Four modes normalized first group flux distribution for the BWR cell test problem. FDM $S P_{3}$ on the left, FDM $S_{8}$ on the right. 


\subsubsection{BWR rod bundle test problem}

The BWR rod bundle case was also introduced in sections 3.8.2 and 4.6.1. The reference solution for this problem was calculated by using DANTSYS. For this solution, the problem was modeled with a mesh of $120 \times 120$ cells and an angular approximation $S_{16}$. FDM $S_{N}, S P_{3}$ and diffusion results were calculated by using a discretization of $120 \times 120$ meshes. Table 5.10 shows a comparison of the results. The results show the same conclusion than the previous problem, the cell centered scheme $S P_{3}$ results are better than diffusion results. Another interesting issue, like in the previous case, could be observed: the accuracy of $S P_{3}$ is approximately the same than $S_{4}$ method, and it differs from $S_{8}$ solution as shown in fig.5.33.

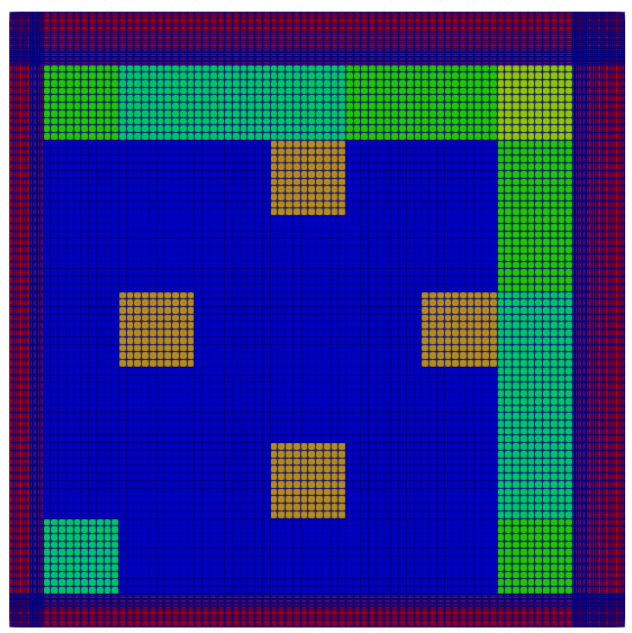

Figure 5.32: BWR rod bundle test material distribution and mesh.

Table 5.10: BWR rod bundle benchmark problem multiplication factor results.

\begin{tabular}{cccccc}
\hline eigenvalue $->$ & 1st & 2nd & 3rd & 4th & pcm $\left(\Delta K_{e f f}\right) 1$ st \\
\hline DANTSYS $S_{16}$ & 1.090330 & - & - & - & - \\
FDM $S_{4}$ & 1.091364 & 0.240292 & 0.235034 & 0.118222 & 54 \\
FDM $S_{6}$ & 1.090922 & 0.241339 & 0.236108 & 0.117371 & 54 \\
FDM $S_{8}$ & 1.090651 & 0.241211 & 0.235994 & 0.117099 & 29 \\
FDM iff..$^{C}$ & 1.088671 & 0.218218 & 0.213061 & 0.093681 & 152 \\
FDM Diff. $\dot{F}^{E}$ & 1.088504 & 0.218241 & 0.213083 & 0.093703 & 167 \\
FDM $S P_{3}^{C}$ & 1.090692 & 0.240399 & 0.235141 & 0.114823 & 33 \\
FDM $S P_{3}^{E}$ & 1.089748 & 0.240371 & 0.235106 & 0.114845 & 53 \\
\hline
\end{tabular}



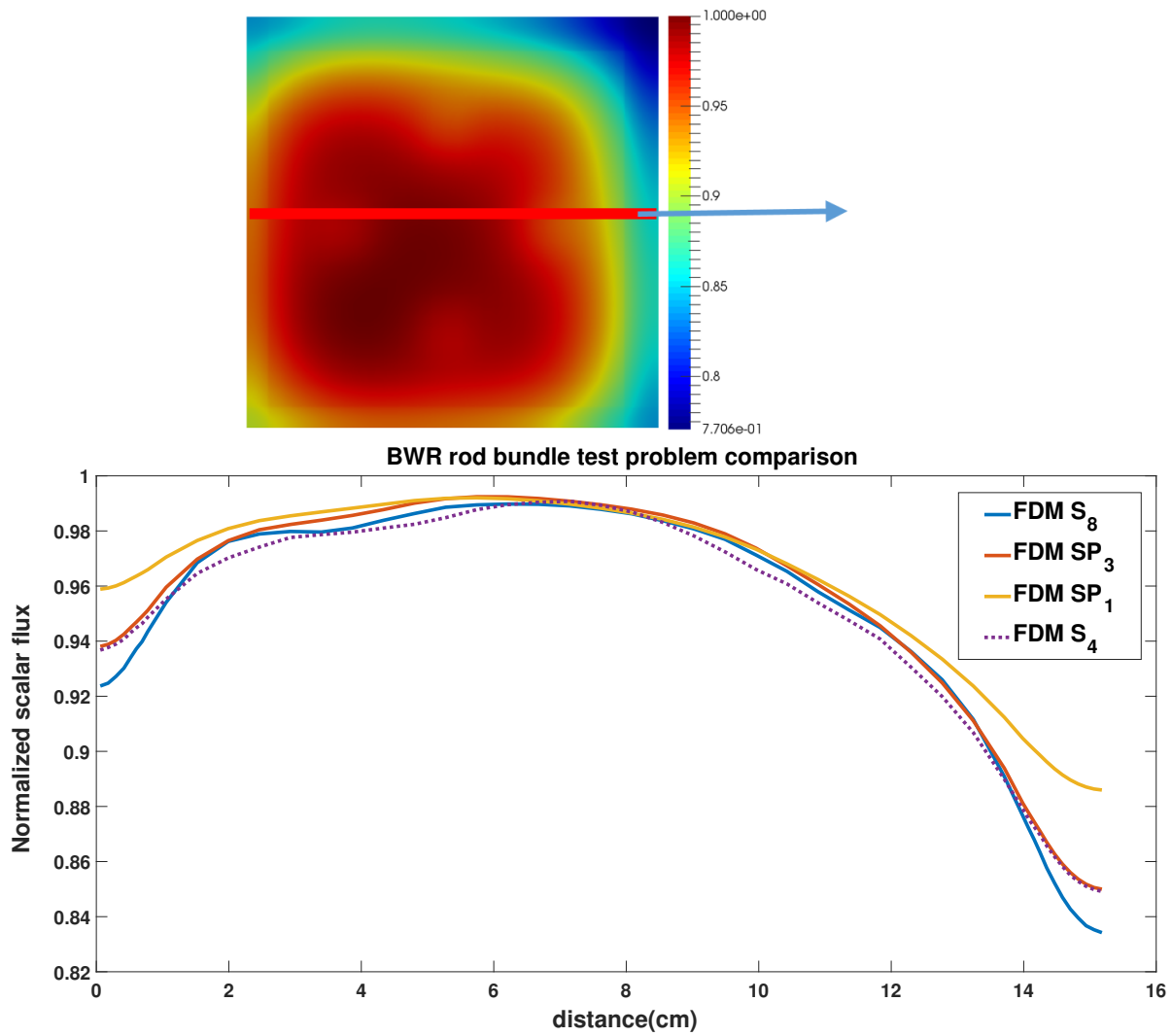

Figure 5.33: BWR rod bundle test: scalar flux distribution for 1st mode by using cell-centered scheme. 

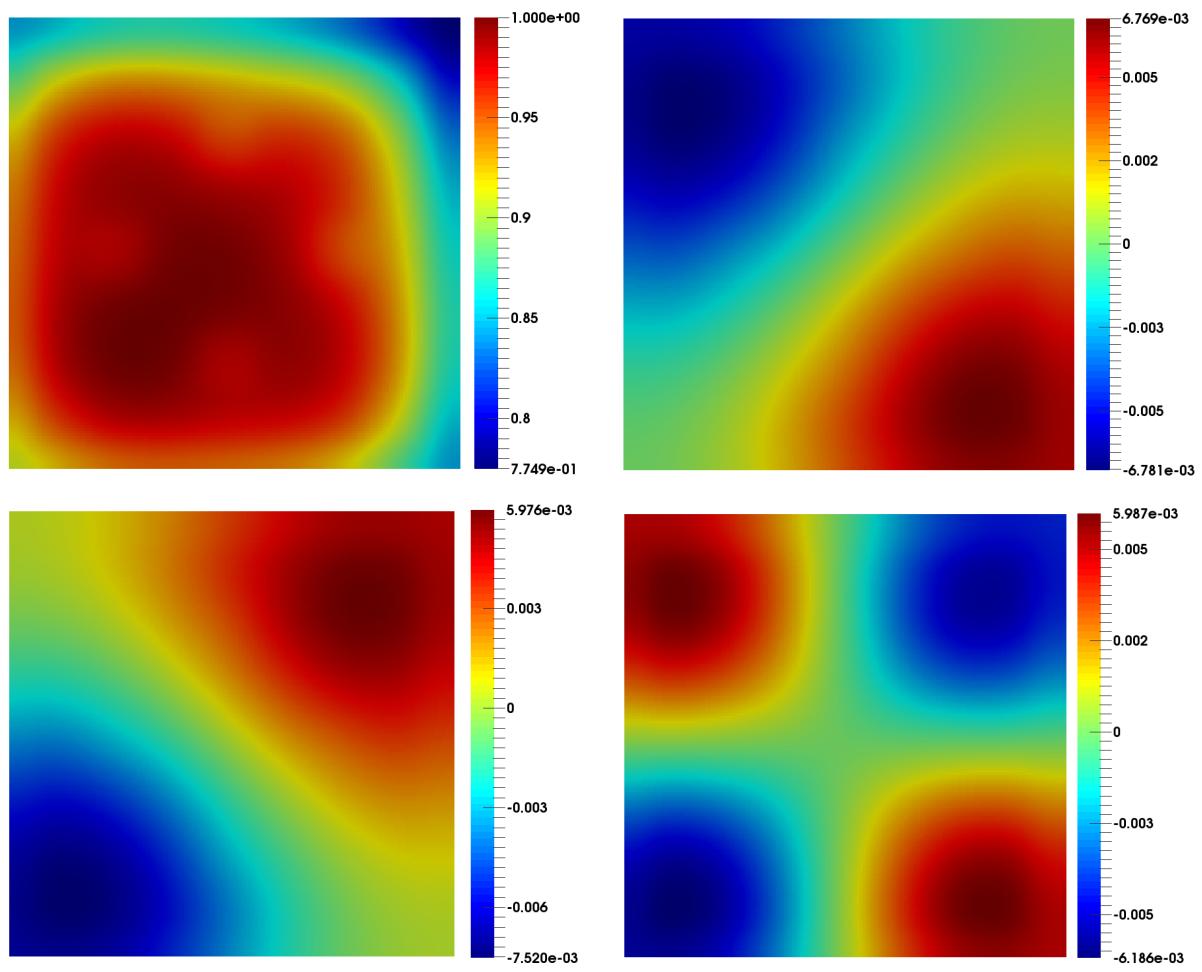

Figure 5.34: Four modes flux distribution for the BWR rod bundle test problem. 


\subsubsection{Two-dimensional C5G7 test problem}

The C5G7 problem was defined in section 3.8.2. The values of $k_{\text {eff }}$ have been compared in table 5.11 for the meshes defined in section 3.8.2, fig.5.35. The next conclusion can be drawn from the results; $k_{e f f}$ values calculated with the edgecentered scheme shows better accuracy than those obtained by the cell-centered scheme, although the maximum percentage error of the power calculated with the cell-centered scheme shows lower values than those obtained by the edgecentered approach. Second, for coarse spatial mesh, the edge-centered scheme has better error cancellation than the cell-centered scheme. A sensitive analysis of the mesh is shown in tables 5.11 and 5.12. The increase of the number of cells is related with a high accuracy regarding to $k_{\text {eff }}$ calculated with cell-centered scheme but no necessarily regarding to the power error.

Fig.5.36 shows the neutron flux distribution of the first eigenvalue for energy groups 1 and 7. The power distribution of the C5G7 problem obtained by FDM $S P_{3}$ is represented in fig.5.37. Fig. 5.38 shows the eigenfluxes comparison between FDM $S P_{3}$ and FDM $S_{4}$. Table 5.13 summarizes the comparison of results obtained by FDM Diffusion and FDM $S P_{3}$ with FEM $S P_{3}$ Vidal-Ferràndiz et al. [2019], FDM $S_{4}$ Morató et al. [2020] and those obtained by MCNP, which provides the reference solution.

Once again, $S_{4}$ provides better results for the eigenvalue as well as lower errors. With respect to $S P_{3}$ and diffusion, no significant differences can be found between the calculated eigenvalues, however the maximum percentage error is always lower for the $S P_{3}$ than for the diffusion.

Table 5.11: $k_{e f f}$ and power comparison for the cell-centered Scheme.

\begin{tabular}{ccccccccc}
\hline & Discret. & N $^{\circ}$ of el. & $k_{\text {eff }}$ & pcm & Max.Perc.Error & AVG & RMS & MRE \\
\hline MCNP & - & - & 1.186550 & - & - & - & - & - \\
SP & $1 \times 1$ & 17424 & 1.180898 & 476 & 7.380 & 2.665 & 2.923 & 2.512 \\
Dif. & $1 \times 1$ & 17424 & 1.182095 & 375 & 6.480 & 2.326 & 2.669 & 2.105 \\
SP & $4 \times 4$ & 191844 & 1.182067 & 377 & 5.666 & 2.463 & 2.654 & 2.331 \\
Dif. & $4 \times 4$ & 191844 & 1.183066 & 294 & 7.385 & 2.223 & 2.807 & 2.015 \\
SP & $6 \times 6$ & 412164 & 1.182089 & 375 & 5.699 & 2.506 & 2.691 & 2.380 \\
Dif. & $6 \times 6$ & 412164 & 1.183032 & 296 & 7.748 & 2.258 & 2.867 & 2.050 \\
SP & $8 \times 8$ & 715716 & 1.183621 & 247 & 5.846 & 2.414 & 2.642 & 2.297 \\
Dif. & $8 \times 8$ & 715716 & 1.183935 & 220 & 8.280 & 2.298 & 2.957 & 2.080 \\
\hline
\end{tabular}


Table 5.12: $k_{e f f}$ and Power comparison for the edge-centered scheme.

\begin{tabular}{ccccccccc}
\hline & Discret. & N $^{\circ}$ of el. & $k_{e f f}$ & pcm & Max.Perc.Error & AVG & RMS & MRE \\
\hline MCNP & - & - & 1.186550 & - & - & - & - & - \\
SP & $1 \times 1$ & 17689 & 1.185380 & 99 & 6.537 & 2.116 & 2.652 & 1.983 \\
Dif. & $1 \times 1$ & 17689 & 1.184963 & 134 & 11.368 & 2.560 & 3.397 & 2.204 \\
SP & $4 \times 4$ & 192721 & 1.184884 & 140 & 5.685 & 2.190 & 2.533 & 2.217 \\
Dif. & $4 \times 4$ & 192721 & 1.184574 & 167 & 10.199 & 2.356 & 3.129 & 2.092 \\
SP $_{3}$ & $6 \times 6$ & 413449 & 1.184468 & 175 & 5.655 & 2.246 & 2.544 & 2.193 \\
Dif. & $6 \times 6$ & 413449 & 1.184347 & 186 & 9.923 & 2.345 & 3.094 & 2.105 \\
SP & $8 \times 8$ & 717409 & 1.183928 & 221 & 5.746 & 2.396 & 2.644 & 2.334 \\
Dif. & $8 \times 8$ & 717409 & 1.184132 & 204 & 9.683 & 2.381 & 3.097 & 2.158 \\
\hline
\end{tabular}
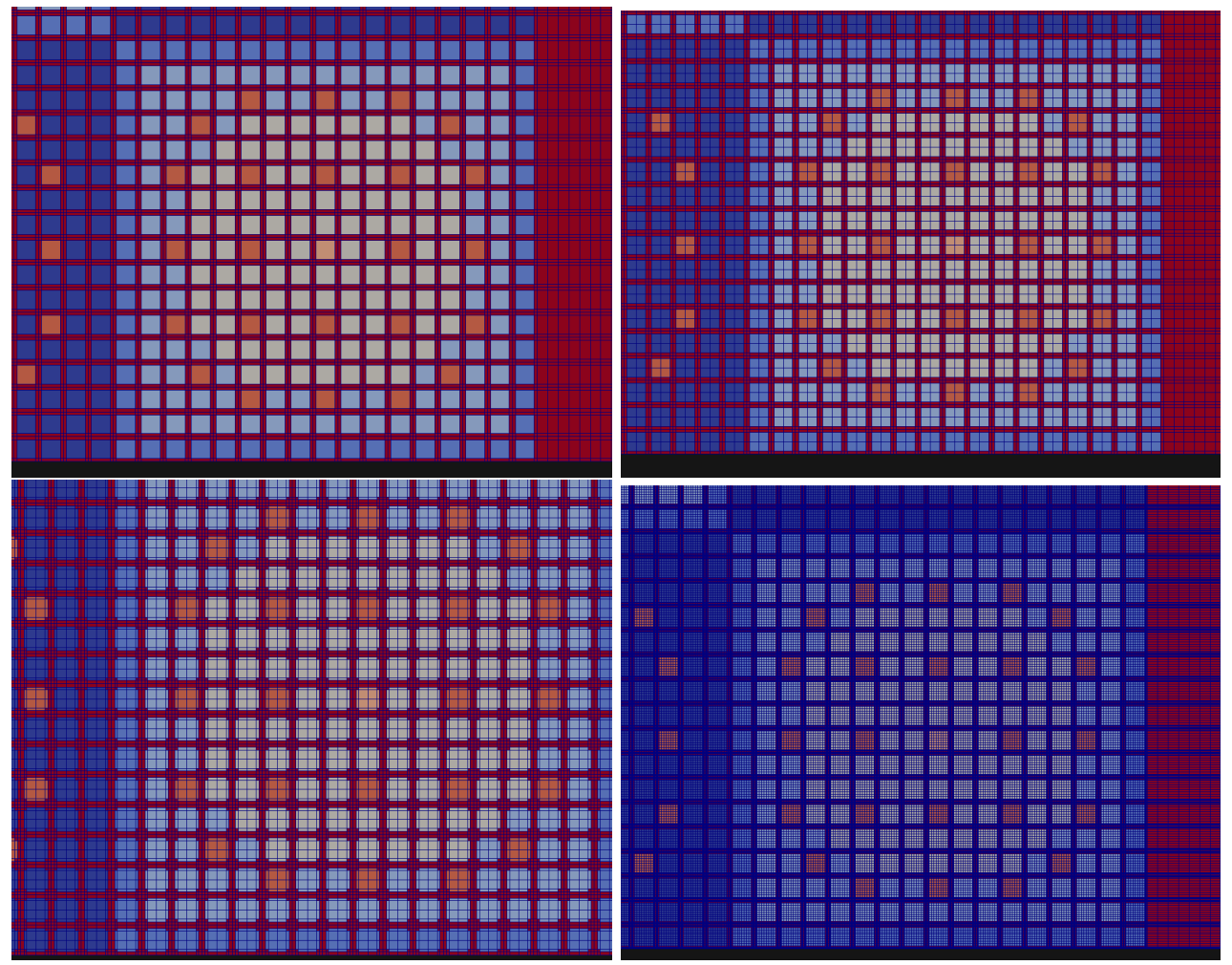

Figure 5.35: Detail of $1 \times 1,4 \times 4,6 \times 6$ and $8 \times 8$ meshes. 

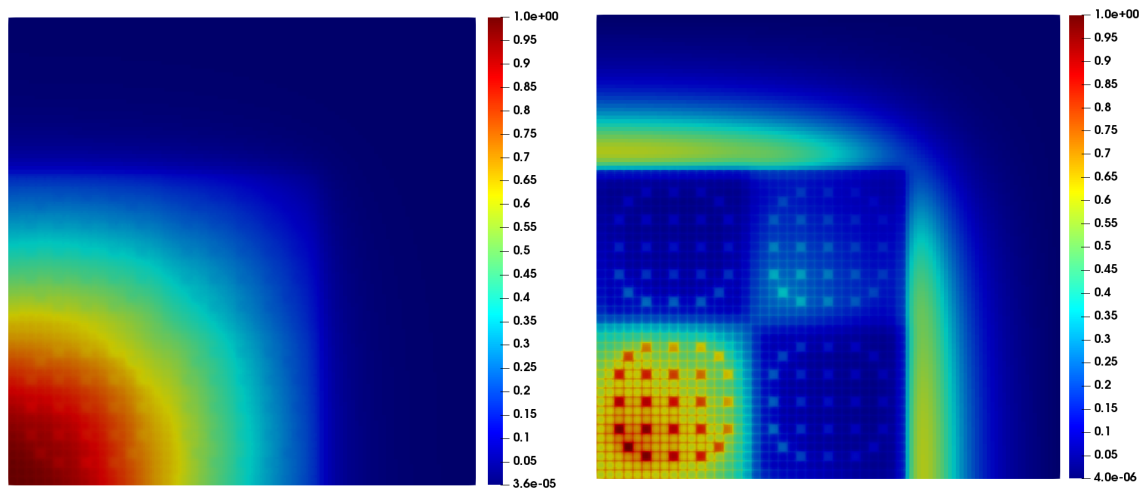

Figure 5.36: FDM $S P_{3}$ Flux 1st mode for energy groups 1 (left) and 7 (right).

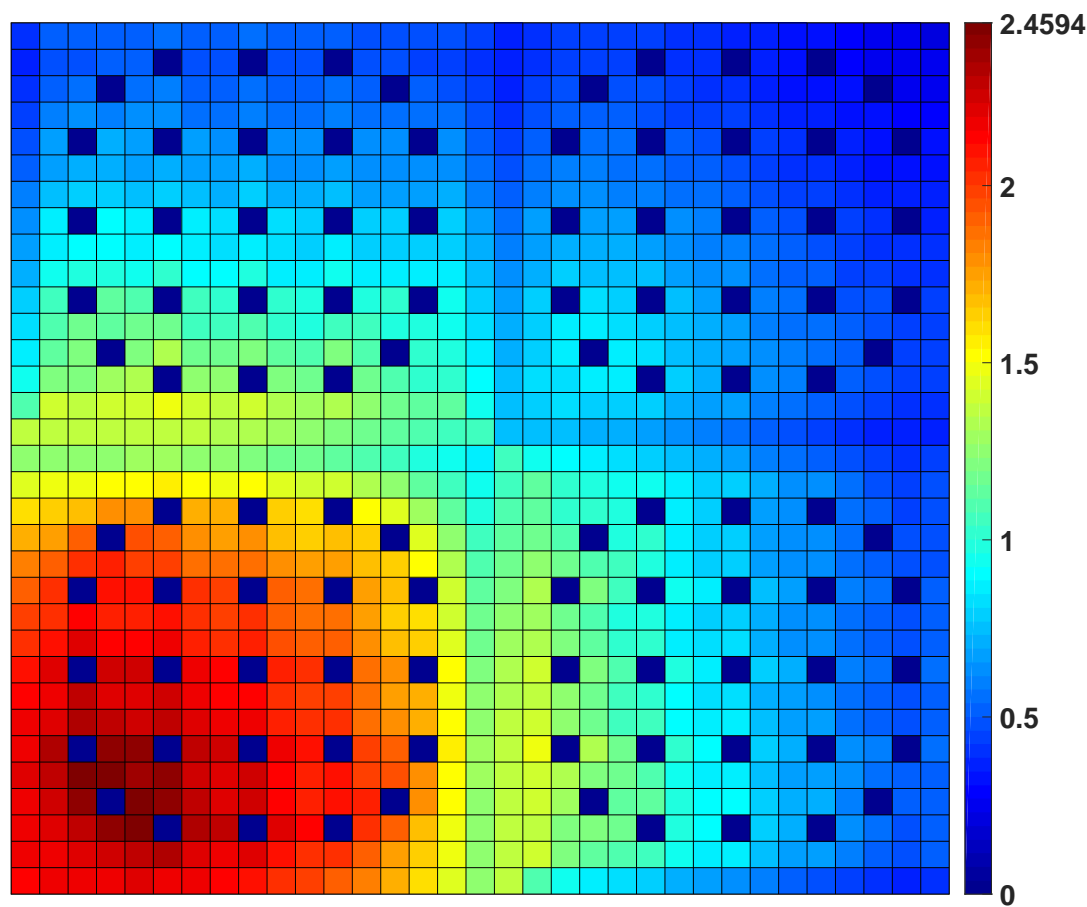

Figure 5.37: Power Distribution of C5G7 problem. 

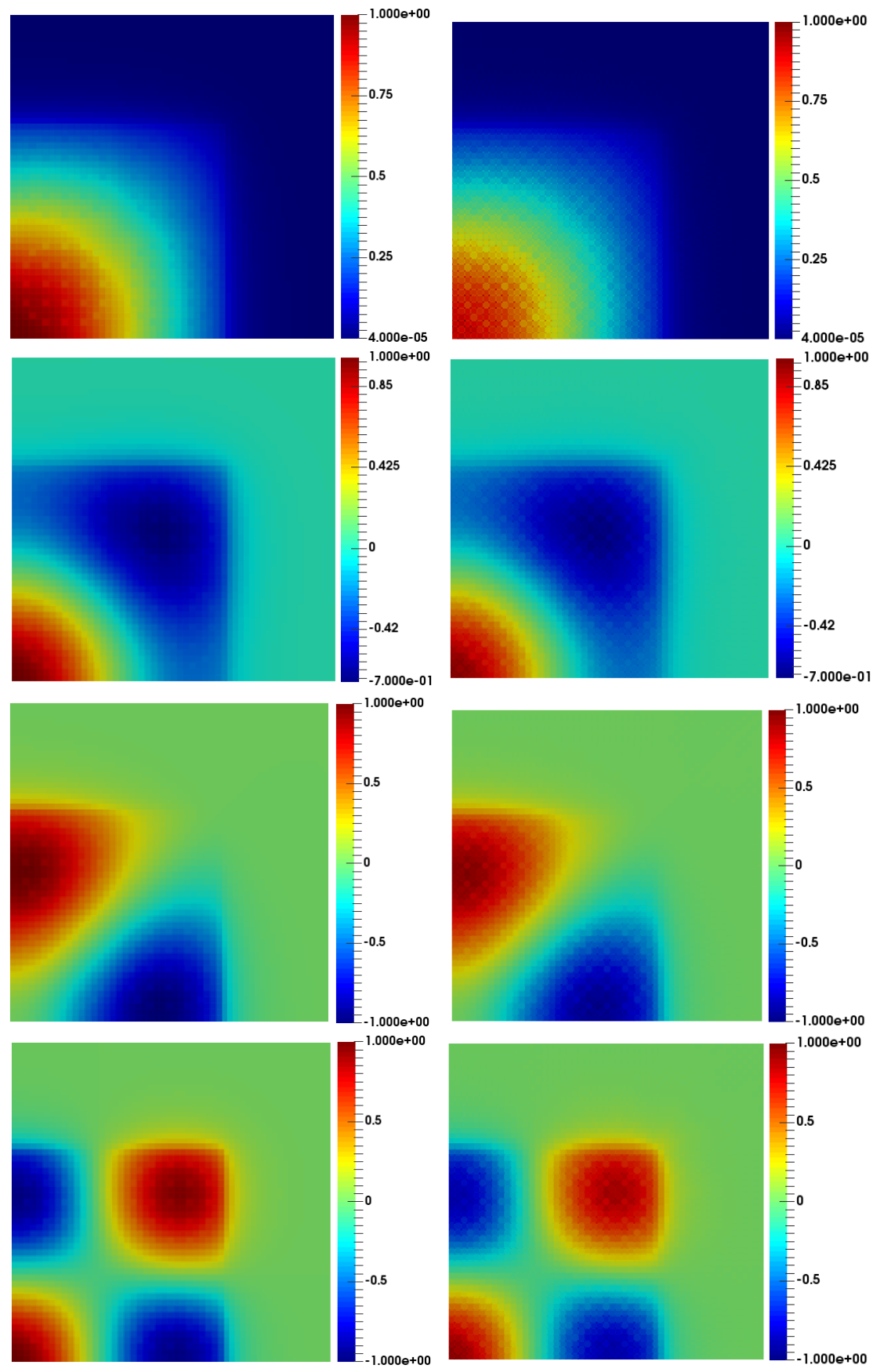

Figure 5.38: Eigenfluxes comparison for the four dominant modes of the $1^{\text {st }}$ energy group. FDM $S P_{3}$ on the left, FDM $S_{4}$ on the right. 
Table 5.13: C5G7 Test problem results. *Results from [Vidal-Ferràndiz et al., 2019].






\section{Computational time}

Table 5.14 shows the computational time required to solve the C5G7 problem with the different approximations developed in this thesis. Although the computational time is not one of the strengths in this thesis, it is important from the point of view of comparing the time for the same test problem with the different approaches. One can appreciate that diffusion and $S P_{3}$ presents better computational times than Discrete ordinates method with orders $S_{2}$ and $S_{4}$. Moreover, the complete C5G7, instead of considering the quarter symmetric core, was also calculated.

Table 5.14: Computational times.

\begin{tabular}{cccccc}
\hline Mesh size & Diffusion & $S P_{3}$ & $S_{2}$ FDM & $S_{2}$ FDM complete & $S_{4}$ FDM \\
\hline $1 \times 1$ & $44 \mathrm{~s}$ & $125 \mathrm{~s}$ & $1382 \mathrm{~s}$ & $27243 \mathrm{~s}$ & $119966 \mathrm{~s}$ \\
$2 \times 2$ & $106 \mathrm{~s}$ & $306 \mathrm{~s}$ & - & - & - \\
$3 \times 3$ & $1211 \mathrm{~s}$ & $3784 \mathrm{~s}$ & - & - & - \\
$4 \times 4$ & $19627 \mathrm{~s}$ & $54275 \mathrm{~s}$ & - & - & - \\
\hline
\end{tabular}

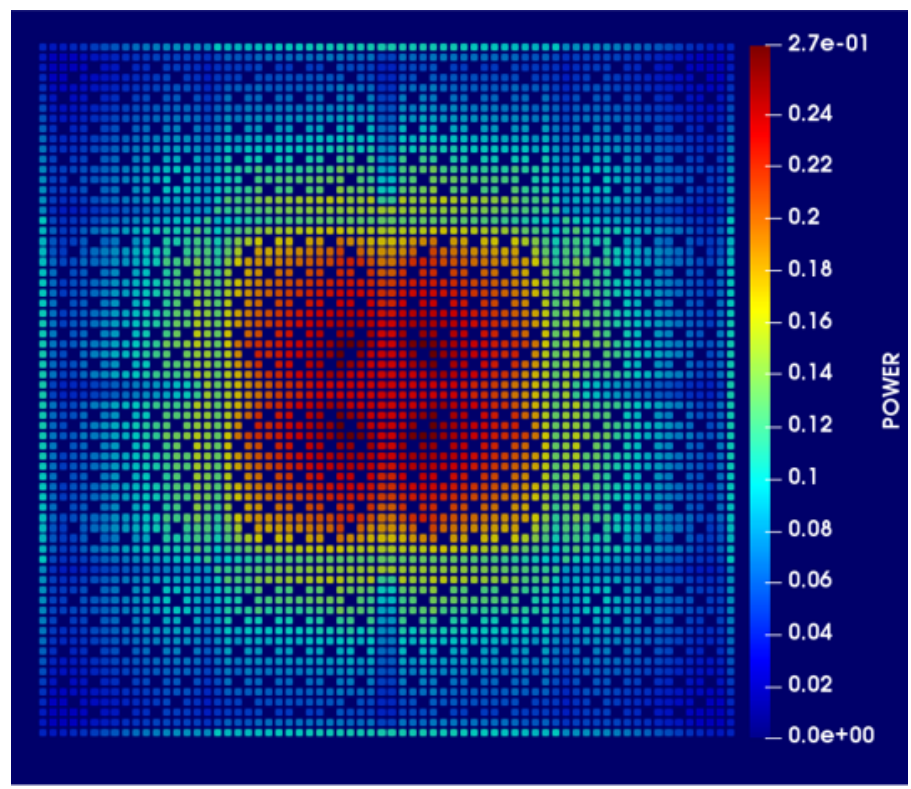

Figure 5.39: Scalar flux first mode $S_{2}$ C5G7 complete. 


\subsection{Numerical results for 3D $S P_{3}$ equations}

\subsubsection{D Homogeneous Reactor}

This reactor is a parallelepiped with dimensions: $100 \mathrm{~cm}$ x $60 \mathrm{~cm} \times 180 \mathrm{~cm}$. It is composed of only one material [Bernal García, 2018]. The cross sections of this material are presented in table5.15 and they are defined for two-energy group, without up-scattering and with fission neutrons produced in the first energy group. Two different meshes are used in the simulation. The first mesh (mesh 1) is $24 \times 16 \times 38$, the total number of elements is 14592 . The second one (mesh 2) is $60 \times 36 \times 102$, the total number of elements is 220320. A representation of both meshes can be found in fig.5.40. Vacuum boundary conditions are applied. The reference solution is calculated with PARTISN by using a $S_{16}$ order quadrature $P_{n}-T_{n}$ for the transport equations and mesh 2. The multiplication factors are compared in table 5.16, including the results obtained with the FDM diffusion method. Fig. 5.41 shows the flux distribution of the first group. It is easy to see that the values obtained by using mesh 2 and the $S P_{3}$ solver, show more accurate $k_{e f f}$ values.

Table 5.15: Homogeneous problem cross-sections. $g=1$ (fast energy group), $g=2$ (thermal energy group).

\begin{tabular}{cccccc}
\hline Group & $\Sigma_{t}$ & $\nu \Sigma_{f}$ & $\Sigma_{s, 1 \rightarrow g}$ & $\Sigma_{s, 2 \rightarrow g}$ & $\chi_{g}$ \\
\hline 1 & $5.2096647 \cdot 10^{-1}$ & $7.72686955 \cdot 10^{-3}$ & $4.95171815 \cdot 10^{-1}$ & - & 1.0 \\
2 & 1.31245720 & $1.55083969 \cdot 10^{-1}$ & $1.60585809 \cdot 10^{-2}$ & 1.20309806 & 0.0 \\
\hline
\end{tabular}
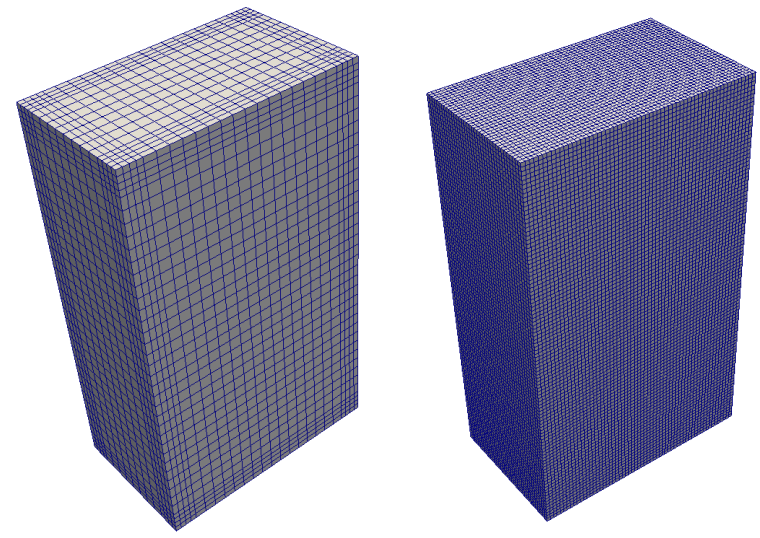

Figure 5.40: Homogeneous reactor meshes. 


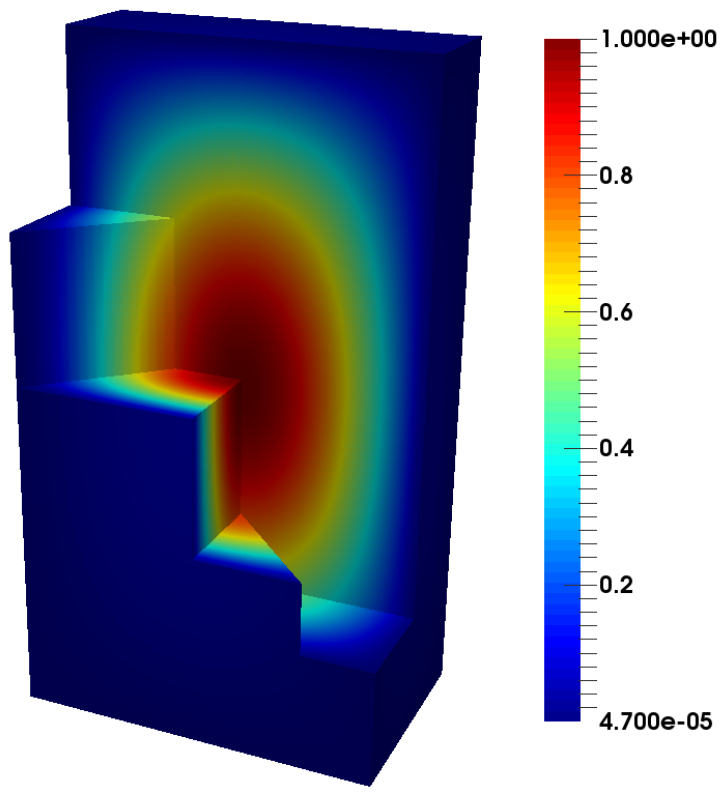

Figure 5.41: Homogeneous reactor flux distribution for eigenvalue 1.

Table 5.16: Multiplication factors for Homogeneous 3D problem.

\begin{tabular}{cccc}
\hline & Order & $K_{\text {eff }}$ & pcm $\left(\Delta K_{\text {eff }}\right)$ \\
\hline Reference PARTISN mesh2 & $S_{16}$ & 1.074001 & - \\
FDM $S P_{3}^{C}$ mesh1 & - & 1.074696 & 64 \\
FDM $S P_{3}^{C}$ mesh2 & - & 1.074141 & 13 \\
FDM Dif. ${ }^{C}$ mesh 1 & - & 1.073891 & 10 \\
FDM Dif. ${ }^{C}$ mesh 2 & - & 1.073373 & 58 \\
FDM $S P_{3}^{E}$ mesh1 & - & 1.075042 & 96 \\
FDM $S P_{3}^{E}$ mesh2 & - & 1.074001 & 0 \\
FDM Dif. ${ }^{E}$ mesh 1 & - & 1.074592 & 55 \\
FDM Dif. ${ }^{E}$ mesh 2 & - & 1.073342 & 61 \\
\hline
\end{tabular}


Boundary conditions comparison

This section shows the differences between the three vacuum boundary approaches explained in section 5.3.2, for the three-dimensional homogeneous problem with mesh 1 . As can be seen in table 5.17, the exact solution is only obtained with the third vacuum boundary condition approach (BC3). The second approach (BC2) differs from exact solution only few figures, while the first approach (BC1) differs considerably. All the results where calculated with the same discretization.

Table 5.17: Values of the first four modes for a 3D homogeneous problem, SP3 boundary condition approaches comparison. $\left(S P_{3}^{C}\right.$ is the $S P_{3}$ code using cell-centered scheme and $B C$ refers to each boundary condition approach).

\begin{tabular}{lccc}
\hline Eig. & FDM $S P_{3}^{C}$ BC3 & FDM $S P_{3}^{C}$ BC2 & FDM $S P_{3}^{C} B C 1$ \\
\hline 1st & 1.074696 & 1.074525 & 1.067359 \\
2nd & 1.049500 & 1.050803 & 1.041714 \\
3rd & 1.010194 & 1.013610 & 1.001778 \\
4th & 1.006673 & 1.006186 & 0.996885 \\
\hline
\end{tabular}

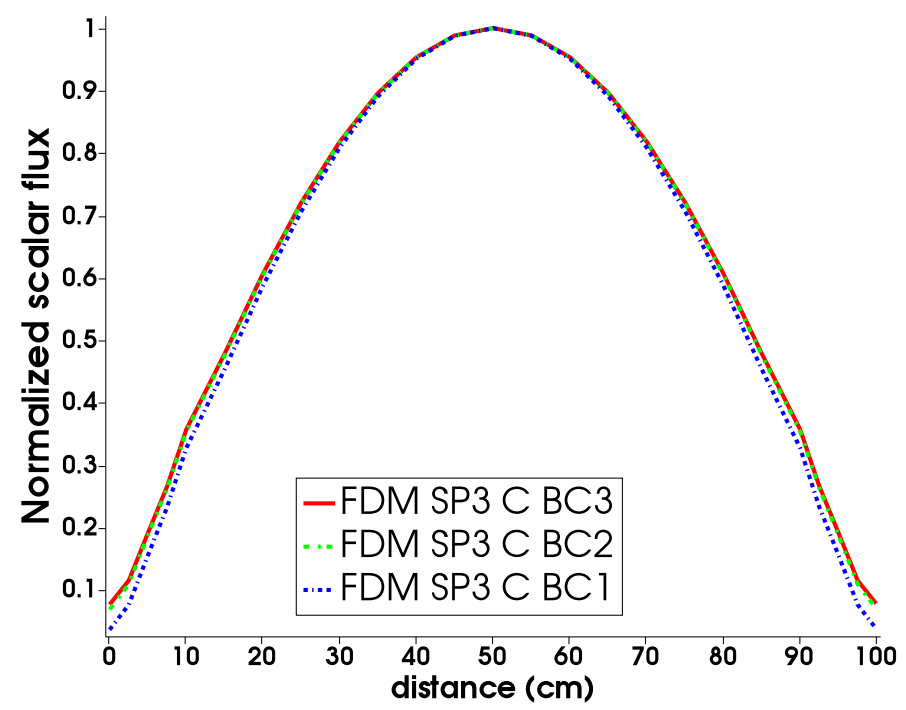

Figure 5.42: Normalized Scalar Flux for Homogeneous 3D reactor for a central line in $x$ axis.

Fig.5.42, 5.43 and 5.44 show the normalized scalar flux calculated with the three different boundary condition approaches. In this case, the difference among the 


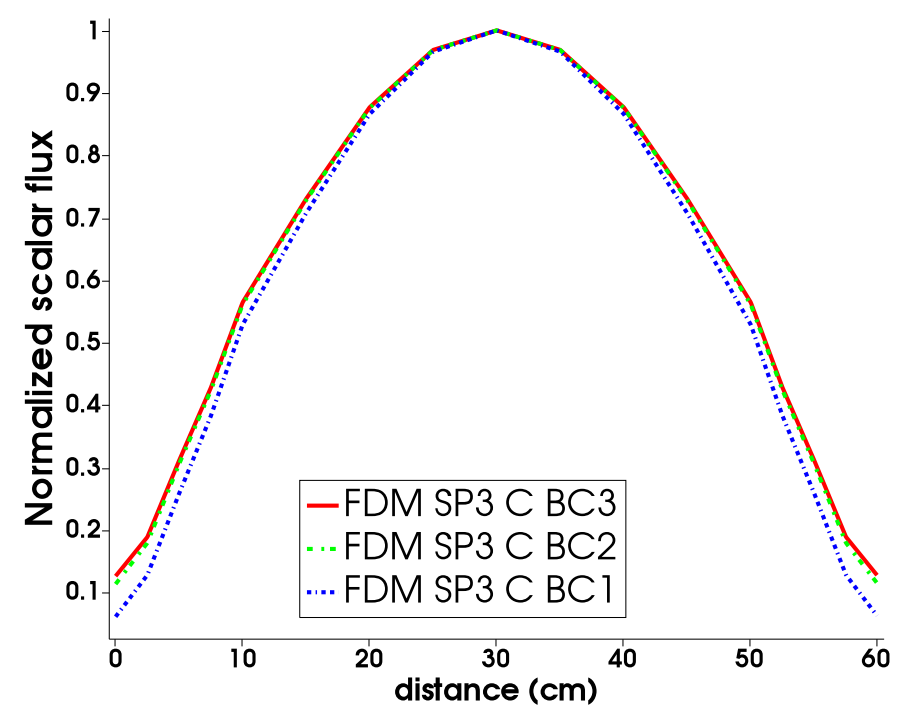

Figure 5.43: Normalized Scalar Flux for Homogeneous 3D reactor for a central line in $y$ axis.

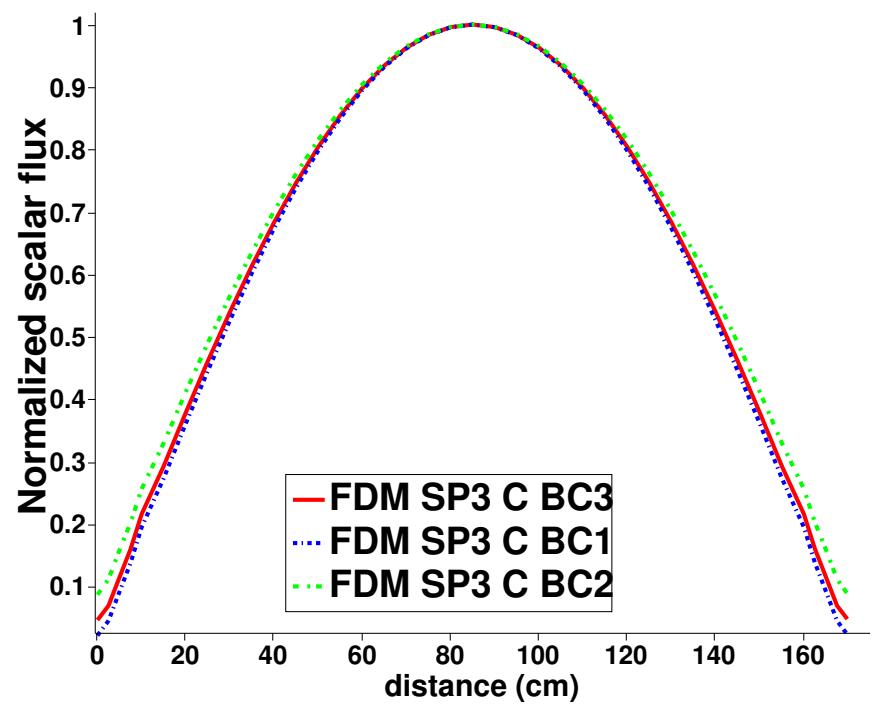

Figure 5.44: Normalized Scalar Flux for Homogeneous 3D reactor for a central line in z axis.

normalized scalar fluxes calculated with the three approaches is not negligible at the boundary. 


\subsubsection{FBR Takeda Benchmark}

The problem considered in this section is a small core model of a Fast Breeder Reactor (FBR). The model is generated with 4 energy groups, and the cross sections are given in [Takeda and Ikeda, 1991]. The dimensions of the problem are $140 \mathrm{~cm} \times 140 \mathrm{~cm} \times 150 \mathrm{~cm}$. The problem is composed of a fuel region, radial and axial blankets and control rod region. In [Takeda and Ikeda, 1991] the problem is generated with symmetry conditions, however in this work the complete problem is considered like in [Capilla et al., 2012]. Vacuum boundary conditions are applied. Fig. 5.45 shows the mesh used ( $144 \times 112 \times 120)$. Two cases of the problem are considered. Case 1: control rods out. Case2: control rods halfinserted. A comparison of the multiplication factors, where the reference value was obtained with Monte Carlo method, is shown in table 5.18. The results of the FDM Diffusion are also included in the previous table. Figs.5.46 and 5.47 show the fluxes distribution of the first energy group for cases 1 and 2. Table 5.18 shows better results for the $S P_{3}$ cell-centered scheme compared with the edgecentered scheme and Diffusion. Figs.5.48 and 5.49 show the fluxes distribution of the fourth energy group for cases 1 and 2.

Table 5.18: Multiplication factors for FBR problem.

\begin{tabular}{ccc}
\hline & $K_{\text {eff }}$ CASE 1 & $K_{\text {eff }}$ CASE 2 \\
\hline Reference Monte-Carlo & 0.973620 & 0.959850 \\
FDM $S P_{3}^{C}$ & 0.964604 & 0.951345 \\
FDM $D i f . .^{C}$ & 0.959346 & 0.945454 \\
FDM $S P_{3}^{E}$ & 0.961727 & 0.947631 \\
FDM $D i f .{ }^{E}$ & 0.956340 & 0.941707 \\
\hline
\end{tabular}

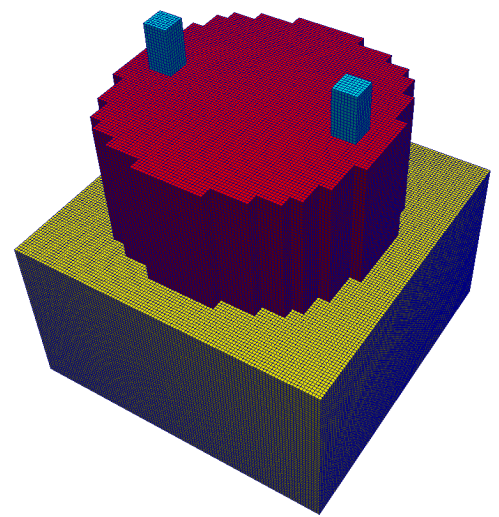

Figure 5.45: FBR reactor discretization (interior visualization of the reactor upper part). 

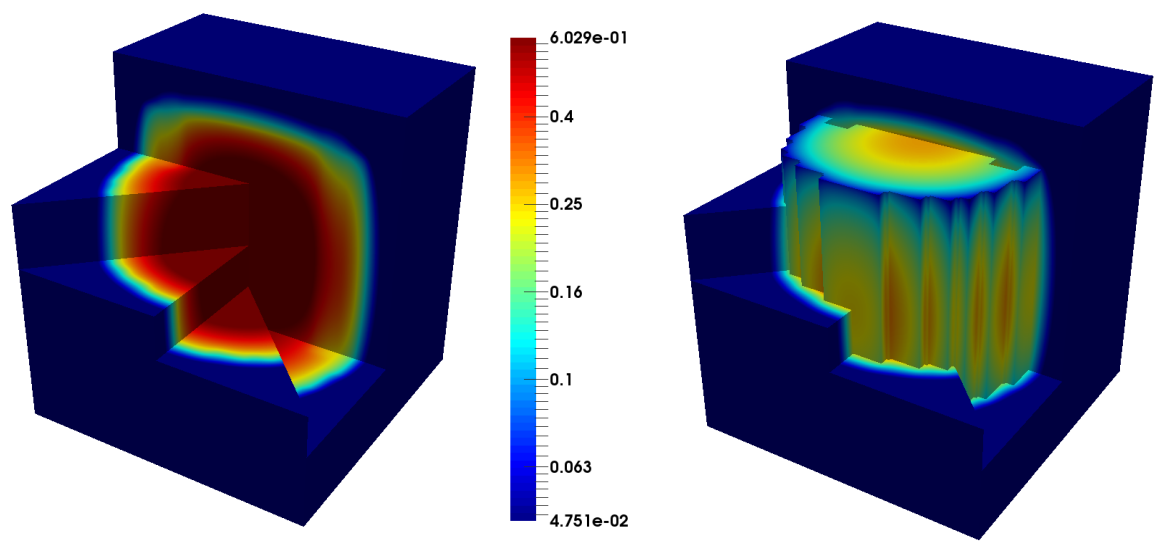

Figure 5.46: Case 1: FBR reactor flux distribution for group 1.
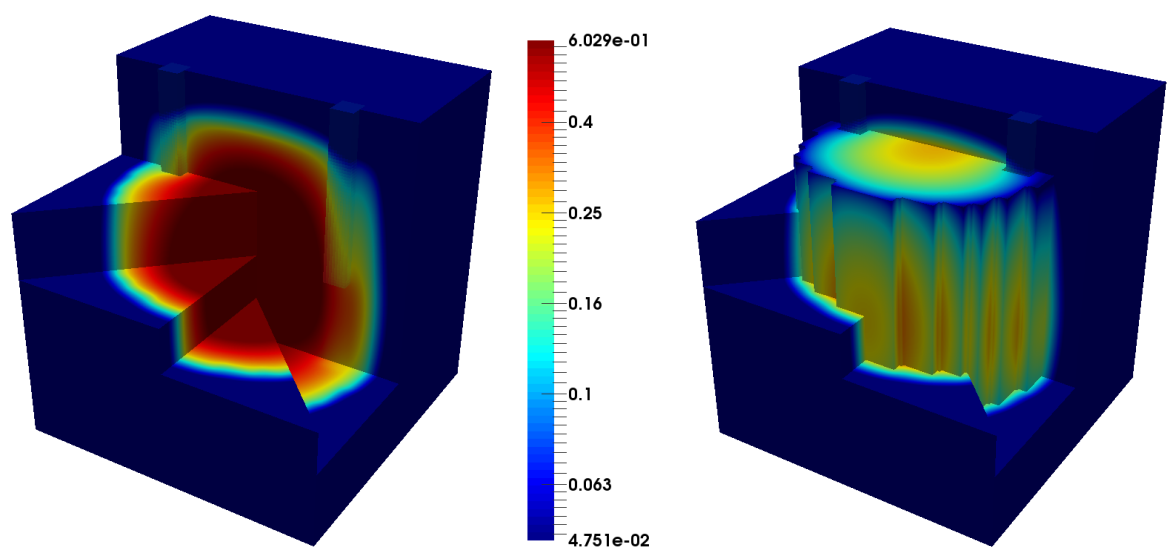

Figure 5.47: Case 2: FBR reactor flux distribution for group 1. 

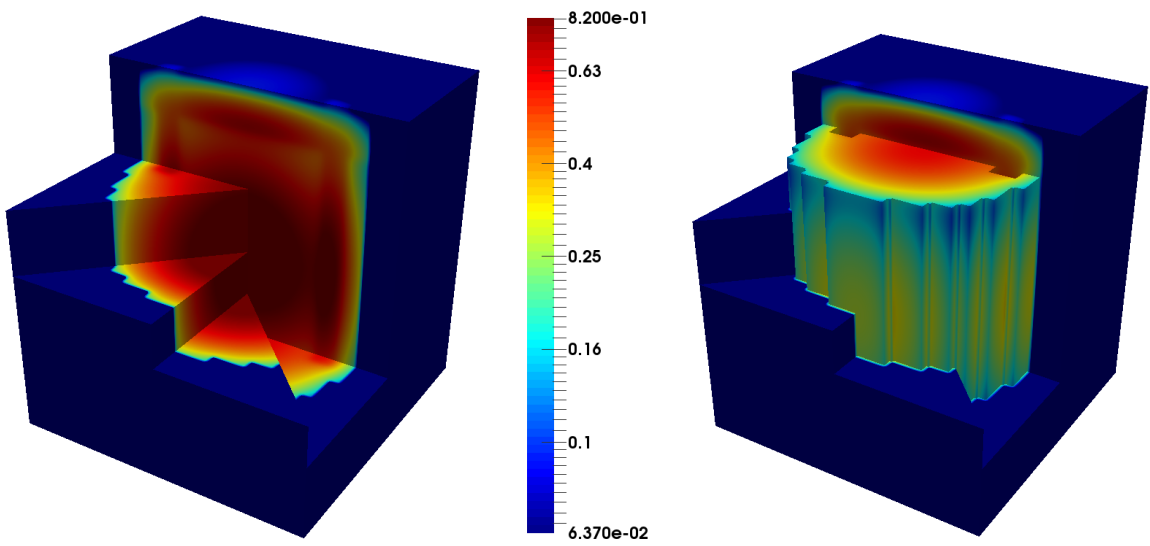

Figure 5.48: Case 1: FBR reactor flux distribution for group 4.
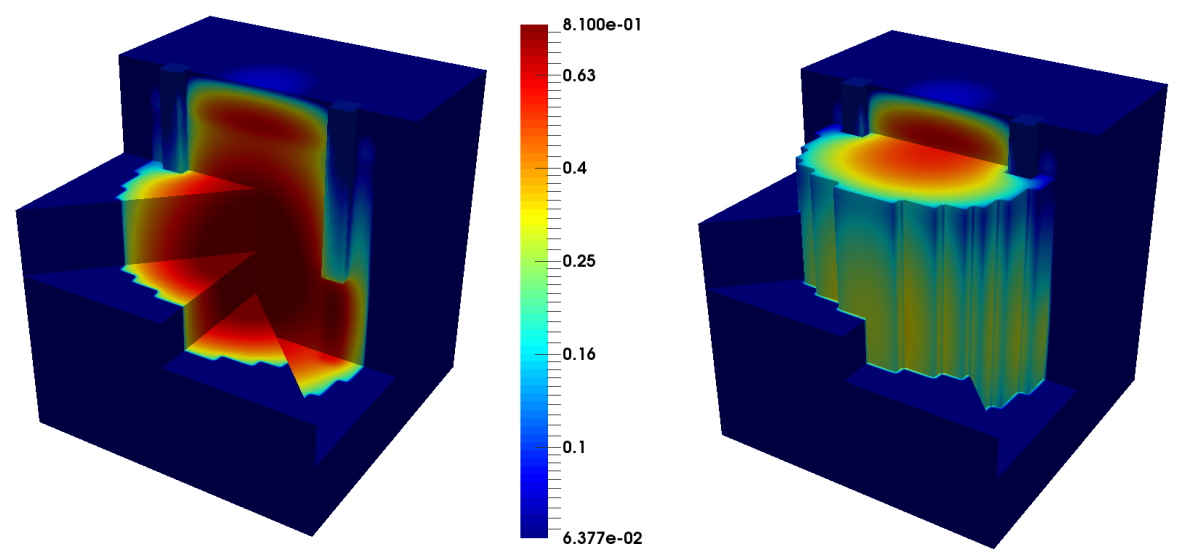

Figure 5.49: Case 2: FBR reactor flux distribution for group 4. 


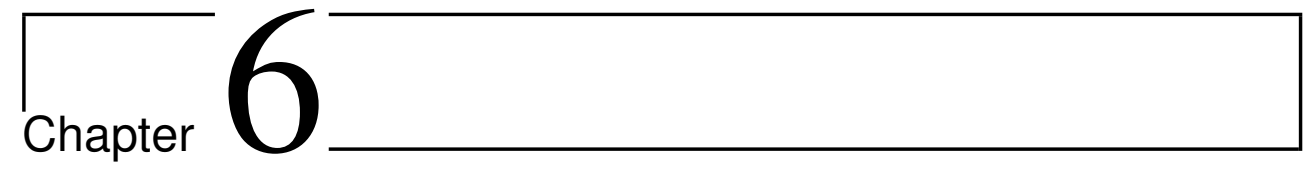

\section{Conclusions}

\subsection{Conclusions}

The achievements in this thesis can be summarized as follows:

1. The main conclusion of the thesis is that important differences can be appreciated with the comparison of the three different approximations of the Neutron Transport Equation implemented in the ValTran code: $S_{N}$, Diffusion and $S_{3}$. Moreover, several modes were compared between the methods. From that, one can conclude that the eigenvectors shape and the sequence in which the lambda modes are calculated does not change with the selected method. Hence, they are not affected by the chosen angular approximation. 
2. A code has been developed for solving the Discrete Ordinates Neutron Transport Equation form the methods explained in chapter 3 with the following features:

- The method solves the one and two-dimensional steady-state multigroup neutron transport equation in Cartesian geometries.

- The Spatial and angular discretization were performed with the Finite Difference Method and the Discrete Ordinates Method, respectively.

- The method is capable of calculating multiple eigenvalues with a simple formulation.

- The method considers both kind of scattering: isotropic and anisotropic.

- Different type of quadrature sets have been implemented and a new $P Q$ quadrature were tested.

- The algorithms have been programmed from scratch in a FORTRAN code called n-DOTEC. This program has been validated with several one-dimensional benchmarks and four two-dimensional benchmarks. The four realistic two-dimensional problems selected are MOX test problem, BWR cell test problem, BWR rod bundle test problem and C5G7 test problem.

- The methodology used in this work shows that n-DOTEC $1 D$ and $2 D$ results are accurate even when anisotropic scattering is considered.

- $n$-DOTEC $2 D$ is capable of modeling cylindrical geometries without spatial homogenization under the simplification of Cartesian geometry. This modeling was tested in the C5G7 benchmark, which obtains good results and it demonstrates that the code is capable of simulating any number of energy groups.

- Two different matrix structures have been performed allowing the use of direct and iterative solvers. The method applies the KrylovSchur method implemented in the SLEPc library to calculate multiple eigenvalues. In addition, SLEPc uses linear algebra algorithms from PETSc to solve linear systems.

- The code can also calculate Neutron Transport Fixed Source Problems. 
3. A Neutron Diffusion Approximation code was developed in chapter 4 with the following features:

- The method solves the one, two and three-dimensional steady-state multi-group Neutron Diffusion Equation in Cartesian geometries.

- The spatial discretization was carried out by means of two different schemes of the Finite Difference Method: cell-centered and edgecentered schemes.

- Several eigenvalues can be calculated with a simple formulation.

- The algorithms have been programmed from scratch in a FORTRAN code called ValTran. The code has been validated with the same benchmarks used for the $S_{N}$ method in order to perform a consistent comparison.

- The methodology used in this work shows that the ValTran results show good agreement with reference values.

- The sensitivity of the mesh was studied.

4. The Simplified Spherical Harmonics Equations explained in chapter 5 were implemented in a code:

- The method is capable of solving a multi-dimensional steady-state multi-group Neutron Diffusion Equation in Cartesian geometries.

- The spatial discretization were performed by means of two versions of the Finite Difference Method: cell-centered and edge-centered schemes.

- This method is capable of calculating multiple eigenvalues and eigenvectors with a simple formulation

- A study of the boundary conditions for the $S P_{3}$ cell-centered scheme was conducted. The exact vacuum boundary condition calculated could not be found elsewhere in the literature, so it could mean that one may reasonably derive incorrect boundary conditions. Therefore, this work is valuable in presenting the correct finite difference vacuum boundary conditions.

- A complete review of the $S P_{3}$ formulation was carried out.

- The algorithms were included into the FORTRAN code called ValTran to use of a simple input for the three approximations explained in this 
thesis: $S_{N}, S P_{3}$ and Diffusion. The code has been validated with the same benchmarks used for the $S_{N}$ and Diffusion methods in order to perform a consistent comparison, as well as, two 3D problems.

- The methodology used in this work shows that the ValTran results show good agreement with reference values.

- The sensitivity of the mesh was studied.

5. The results shown in this thesis imply that there are important neutron flux differences between the three methods implemented: Diffusion, $S_{3}$ and $S_{N}$. So, it is important to know what method is suitable depending on the calculation, accuracy and computational cost.

\subsection{Scientific contributions}

Two principal contributions were published related with this thesis, which are the first two works mentioned in the next section: "Lambda modes comparison for different approximations of the Neutron Transport Equation: Diffusion, SN and SP3" and "Calculation of Lambda modes of the multi-group neutron transport equation using the discrete ordinates and Finite Difference Method".

However, the interest of the author in the medical applications of the nuclear technology and radiation protection leads the author to research and publish in parallel other important contributions related with this field. The future idea is to unify both research tracks with a deeper development of the Neutron Transport Fixed Source Problem, because these calculations can be very useful for medical and radiation protection applications. These kind of problems are very common in shielding calculations to calculate the least amount of shielding material minimizing the neutron or photon dose [Morató et al., 2015]. This methodology could serve to design radiotherapy bunkers [Juste et al., 2018a] or to know what components of the radiotherapy treatment devices receive more radiation than other to select the adequate materials [Juste et al., 2018b, 2017, Morató et al., 2016].

Thus, all the works developed and all contributions in conferences generated by the author during the time that his doctoral thesis has lasted are listed below.

Both developed codes, ValTran and n-DOTEC, will be available for the public in the Nuclear Energy Agency (NEA) Data Bank or similar repositories in the near future. 


\subsubsection{Publications in International Journals}

\begin{aligned} & \hline 1. Title: $\begin{array}{l}\text { Lambda modes comparison for different approximations of the Neutron } \\ \text { Transport Equation: Diffusion, SN and SP3. }\end{array} \\ &$\hline Journal: Annals of Nuclear Energy \\ & Year: 2020 \\ & Authors: $\begin{array}{l}\text { Morató-Rafet, Sergio; Kochunas, Brendan; Miró Herrero, Rafael; Verdú } \\ \text { Martín, Gumersindo Jesús; Larsen, Edward.W.; Downar, Thomas }\end{array} \\ &$\hline\end{aligned}

2. Title: Calculation of Lambda modes of the multi-group neutron transport equation using the discrete ordinates and Finite Difference Method.

Journal: Annals of Nuclear Energy

Year: 2020

Authors: $\quad$ Morató-Rafet, Sergio; Bernal-Garcia, Alvaro; Miró Herrero, Rafael; Jose E. Roman; Verdú Martín, Gumersindo Jesús
3. Title: Development of a reconstruction methodology for the X-Ray spectrum of a medical LinAc positioning flat panel.
Journal: Radiation Physics and Chemistry
Year: 2020
Authors: Juste Vidal, Belen Jeanine; Morató-Rafet, Sergio; Miró Herrero, Rafael; Ana Isabel Prieto; Verdú Martín, Gumersindo Jesús; R. Genovés; Jose Gimeno- Olmos
4. Title: Monte Carlo code application to the study of 3D neutrons distribution in a radiotherapy bunker and validation with experimental measurements.
Journal: Nuclear Instruments and Methods in Physics Research
Year: 2020
Authors: Juste Vidal, Belen Jeanine; Morató-Rafet, Sergio; C. García; R. Miró; Verdú Martín, Gumersindo Jesús
5. Title: Prostate cancer Monte Carlo dose model with (177)Lutetium and (125)Iodine treatments.
Journal: Radiation Physics and Chemistry
Year: 2020
Authors: Juste Vidal, Belen Jeanine; Miró Herrero, Rafael; Verdú Martín, Gumersindo Jesús; Morató-Rafet, Sergio; S. Peris




\section{Title: Evaluation of the response of a Bonner Sphere Spectrometer with a (LiI)} Li-6 detector using 3D meshed MCNP6.1.1 models.

Journal: Radiation Physics and Chemistry

Year: 2019

Authors: Morató-Rafet, Sergio; Juste Vidal, Belen Jeanine; Miró Herrero, Rafael; Verdú Martín, Gumersindo Jesús; Guàrdia, V

7. Title: MCNP6 unstructured mesh application to estimate the photoneutron distribution and induced activity inside a linac bunker.

Journal: Radiation Physics and Chemistry

Year: 2017

Authors: $\quad$ B. Juste; S. Morató; R. Miró; Verdú Martín, Gumersindo Jesús; Sergi Díez Domingo

\begin{tabular}{rrl}
\hline 8. & Title: & VARIAN CLINAC $6 \mathrm{MeV}$ Photon Spectra Unfolding using a Monte Carlo \\
& Meshed Model. \\
\hline Journal: & EPJ Web of Conferences \\
Year: & 2017 \\
Authors: & Morató-Rafet, Sergio; Juste Vidal, Belen Jeanine; Miró Herrero, Rafael; \\
& Verdú Martín, Gumersindo Jesús \\
\hline
\end{tabular}

\subsubsection{International conferences contributions}

\begin{aligned} \hline 1. Title: & $\begin{array}{l}\text { Brachytherapy prostate treatment planning using Monte Carlo simula- } \\ \text { tion. }\end{array} \\$\hline Conference: & Women in Nuclear Global Annual Conference (WIN) \\ Year: & 2019 \\ Authors: & $\begin{array}{l}\text { Juste Vidal, Belen Jeanine; Morató-Rafet, Sergio; Miró Herrero, Rafael; } \\ \text { Verdú Martín, Gumersindo Jesús }\end{array}\end{aligned}$

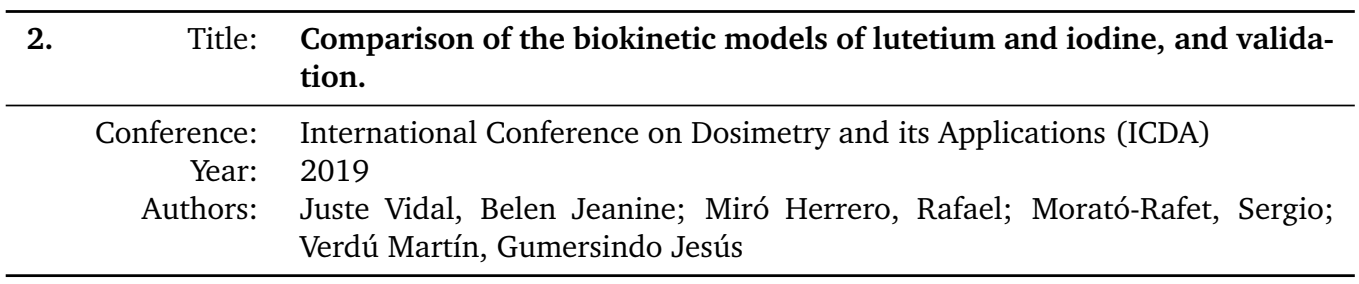




\begin{aligned} & \hline 3. Title: $\begin{array}{l}\text { 3D dosimetry based on SPECT/CT: Evaluation for Lutetium Therapy us- } \\ \text { ing Monte Carlo simulation and the NEMA phantom. }\end{array} \\ &$\hline Conference: International Conference on Dosimetry and its Applications (ICDA) \\ & Year: $\begin{array}{l}2019 \\ \text { Authors: }\end{array} \\ & \begin{array}{l}\text { Juste Vidal, Belen Jeanine; Morató-Rafet, Sergio; Miró Herrero, Rafael; } \\ \text { I.Torres; Verdú Martín, Gumersindo Jesús }\end{array} \\ &$\hline\end{aligned}

\begin{aligned} & \hline $4 . \quad$ Title: $\begin{array}{l}\text { Development of a reconstruction methodology for the X-Ray spectrum } \\ \text { of a medical Linac positioning flat panel. }\end{array} \\ &$\hline Conference: International Symposium on Radiation Physics (ISRP) \\ & Year: $\begin{array}{l}2018 \\ \text { Authors: }\end{array} \\ & \begin{array}{l}\text { Juste Vidal, Belen Jeanine; Morató-Rafet, Sergio; Miró Herrero, Rafael; } \\ \text { I.Torres; Verdú Martín, Gumersindo Jesús }\end{array} \\ &$\hline\end{aligned}

\begin{tabular}{rll}
\hline 5. & Title: & n-DOTEC results for analytical benchmark 1D and C5G7 benchmark 2D. \\
\hline Conference: & $\begin{array}{l}\text { International Conference on Physics and Technology of Reactors and Appli- } \\
\text { cations (PHYTRA) }\end{array}$ \\
Year: & 2018 \\
Authors: & $\begin{array}{l}\text { Morató-Rafet, Sergio; Bernal-Garcia, Alvaro; Jose E. Roman; Miró Herrero, } \\
\text { Rafael; Verdú Martín, Gumersindo Jesús }\end{array}$ \\
\hline
\end{tabular}

\begin{aligned} & \hline $6 . \quad$ Title: $\begin{array}{l}\text { Measurement and Monte Carlo simulation of 3d neutrons distribution } \\ \text { in a radiotherapy bunker. }\end{array} \\ &$\hline Conference: $\begin{array}{l}\text { Topical Meeting of the Radiation Protection and Shielding Division of the } \\ \text { American Nuclear Society (RPSD) }\end{array} \\ &$ Year: 2018 \\ & Authors: Morató-Rafet, Sergio; Juste Vidal, Belen Jeanine; Miró Herrero, Rafael; \\ & Verdú Martín, Gumersindo Jesús \\ & \hline\end{aligned}

\begin{aligned} \hline 7. Title: & $\begin{array}{l}\text { Neutron production in the beryllium filter of a linac using } 6 \text { mev treat- } \\ \text { ment beam. }\end{array} \\$\hline Conference: & $\begin{array}{l}\text { Topical Meeting of the Radiation Protection and Shielding Division of the } \\ \text { American Nuclear Society (RPSD) }\end{array} \\$ Year: & 2018 \\ Authors: & $\begin{array}{l}\text { Alejandra Salvat; Morató-Rafet, Sergio; Juste Vidal, Belen Jeanine; Miró Her- } \\ \text { rero, Rafael; Verdú Martín, Gumersindo Jesús }\end{array} \\ & \end{aligned}$




\begin{tabular}{|c|c|}
\hline Title: & $\begin{array}{l}\text { Brachytherapy organ dose estimation using Monte Carlo simulations of } \\
\text { realistic patient models. }\end{array}$ \\
\hline Conference: & $\begin{array}{l}\text { Annual International Conference of the IEEE Engineering in Medicine and } \\
\text { Biology Society (EMBC) }\end{array}$ \\
\hline Year: & 2018 \\
\hline Authors: & $\begin{array}{l}\text { Morató-Rafet, Sergio; Juste Vidal, Belen Jeanine; S. Peris; Miró Herrero, } \\
\text { Rafael; Verdú Martín, Gumersindo Jesús; F.Ballester; J. Vijande }\end{array}$ \\
\hline
\end{tabular}

9. Title: Study of the photoneutron generation caused by a LinAc Beryllium window with a $6 \mathrm{MeV}$ treatment beam.

Conference: Annual International Conference of the IEEE Engineering in Medicine and Biology Society (EMBC)

Year: 2018

Authors: Juste Vidal, Belen Jeanine; Morató-Rafet, Sergio; Alejandra Salvat; Miró Herrero, Rafael; Verdú Martín, Gumersindo Jesús

10. Title: A Gauss-Legendre Product Quadrature for the Neutron Transport Equation.

Conference: Mathematical Modelling in Engineering \& Human Behaviour (formerly: Mathematical Models of Addictive Behaviour, Medicine and Engineering)

Year: 2018

Authors: Bernal-Garcia, Alvaro; Morató-Rafet, Sergio; Miró Herrero, Rafael; Verdú Martín, Gumersindo Jesús

11. Title: Monte Carlo code application to the study of 3d neutrons distribution in a radiotherapy bunker and validation with experimental measurements.

Conference: Symposium on Radiation measurements and Applications (SORMA)

Year: 2018

Authors: Juste Vidal, Belen Jeanine; Morató-Rafet, Sergio; Miró Herrero, Rafael; Verdú Martín, Gumersindo Jesús

\begin{tabular}{|c|c|}
\hline Title: & $\begin{array}{l}\text { Response matrix calculation of a Bonner sphere spectrometer using } \\
\text { ENDF/B-VII libraries. }\end{array}$ \\
\hline Conference: & 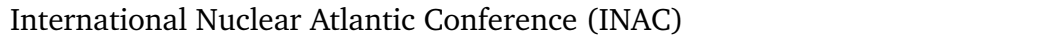 \\
\hline Year: & 2017 \\
\hline Authors: & $\begin{array}{l}\text { Morató-Rafet, Sergio; Juste Vidal, Belen Jeanine; Miró Herrero, Rafael; } \\
\text { Verdú Martín, Gumersindo Jesús; V. Guàrdia }\end{array}$ \\
\hline
\end{tabular}




\begin{tabular}{|c|c|c|}
\hline 13. & Title: & Dose Calculation in Computerized Tomography . \\
\hline & Conference: & $\begin{array}{l}\text { Annual International Conference of the IEEE Engineering in Medicine and } \\
\text { Biology Society (EMBC) }\end{array}$ \\
\hline & Year: & 2017 \\
\hline & Authors: & $\begin{array}{l}\text { Morató-Rafet, Sergio; C. García; Juste Vidal, Belen Jeanine; Miró Herrero, } \\
\text { Rafael; Verdú Martín, Gumersindo Jesús }\end{array}$ \\
\hline
\end{tabular}

\begin{aligned} & \hline 14. Title: $\begin{array}{l}\text { Computed Tomography Dose Estimation Using Monte Carlo Simulation } \\ \text { (MC-GPU). }\end{array} \\ &$\hline Conference: $\begin{array}{l}\text { International Topical Meeting on Industrial Radiation and Radioisotope Mea- } \\ \text { surement Applications (IRRMA) }\end{array} \\ &$ Year: 2017 \\ & Authors: Morató-Rafet, Sergio; Juste Vidal, Belen Jeanine; Miró Herrero, Rafael; \\ & Verdú Martín, Gumersindo Jesús \\ & \hline\end{aligned}

\begin{aligned} & \hline 15. Title: $\begin{array}{l}\text { Evaluation of the Response of a Bonner Sphere Spectrometer with a 6LiI } \\ \text { detector using 3D meshed MCNP6.1.1 models. }\end{array} \\ &$\hline Conference: $\begin{array}{l}\text { International Topical Meeting on Industrial Radiation and Radioisotope Mea- } \\ \text { surement Applications (IRRMA) }\end{array} \\ &$ Year: $\begin{array}{l}2017 \\ \text { Authors: }\end{array} \\ &$ Morató-Rafet, Sergio; Juste Vidal, Belen Jeanine; Miró Herrero, Rafael; \\ & Verdú Martín, Gumersindo Jesús \\ & \hline\end{aligned}

\begin{tabular}{rrl} 
16. Title: & Advantages of using MCNP6 meshing tools: Three medical cases studies. \\
\hline Conference: & IEEE Nuclear Science Symposium and Medical Imaging Conference \\
& (NSS/MIC) \\
Year: & 2016 \\
Authors: & $\begin{array}{l}\text { Morató-Rafet, Sergio; Juste Vidal, Belen Jeanine; Miró Herrero, Rafael; } \\
\text { Verdú Martín, Gumersindo Jesús }\end{array}$ \\
\hline
\end{tabular}

\begin{tabular}{|c|c|c|}
\hline 17. & Title: & $\begin{array}{l}\text { Monte Carlo Dose Estimation in Patient-Specific Volume Mesh From CT } \\
\text { Images. }\end{array}$ \\
\hline & Conference: & $\begin{array}{l}\text { International Congress on Image and Signal Processing, BioMedical Engi- } \\
\text { neering and Informatics (CISP-BMEI) }\end{array}$ \\
\hline & Year: & 2016 \\
\hline & Authors: & $\begin{array}{l}\text { Morató-Rafet, Sergio; Miró Herrero, Rafael; Juste Vidal, Belen Jeanine; } \\
\text { Verdú Martín, Gumersindo Jesús; Agustín Santos Serra }\end{array}$ \\
\hline
\end{tabular}


18. Title: VARIAN CLINAC $6 \mathrm{MeV}$ Photon Spectra Unfolding using a Monte Carlo Meshed Model.

Conference: International Conference on Radiation Shielding (ICRS)

Year: 2016

Authors: Morató-Rafet, Sergio; Juste Vidal, Belen Jeanine; Miró Herrero, Rafael; Verdú Martín, Gumersindo Jesús

19. Title: Experimental Validation of Neutron Activation Simulation of a Varian Medical Linear Accelerator.

Conference: Annual International Conference of the IEEE Engineering in Medicine and Biology Society (EMBC)

Year: 2016

Authors: Morató-Rafet, Sergio; Juste Vidal, Belen Jeanine; Miró Herrero, Rafael; Verdú Martín, Gumersindo Jesús; Sergio Díez Domingo

\section{Title: Dose Calculation from Photoneutrons emitted in Radiotherapy Treat-} ments by means of MCNP6 Simulation and Unstructured Mesh.

Conference: IEEE Nuclear Science Symposium and Medical Imaging Conference

Year: 2016

Authors: Morató-Rafet, Sergio; Juste Vidal, Belen Jeanine; Miró Herrero, Rafael; Verdú Martín, Gumersindo Jesús

\subsubsection{National conferences contributions}

\begin{aligned} & \hline 1. Title: $\begin{array}{l}\text { Comparación de diferentes aproximaciones del Transporte Neutrónico: } \\ \text { Difusión, SP3 y SN. }\end{array} \\ &$\hline Conference: $45^{\text {a }}$ Reunión Anual de la Sociedad Nuclear Española \\ & Year: 2019 \\ & Authors: $\begin{array}{l}\text { Morató-Rafet, Sergio; Vidal-Ferràndiz, Antoni; Bernal-Garcia, Alvaro; Miró } \\ \text { Herrero, Rafael; Verdú Martín, Gumersindo Jesús }\end{array} \\ &$\hline\end{aligned}

2. Title: Utilización de la simulación MC para el estudio de las dosis recibidas por los pacientes de cáncer de próstata con dos diferentes tratamientos.

Conference: $\quad 45^{\mathrm{a}}$ Reunión Anual de la Sociedad Nuclear Española

Year: 2019

Authors: Juste Vidal, Belen Jeanine; Miró Herrero, Rafael; Morató-Rafet, Sergio; Verdú Martín, Gumersindo Jesús 


\begin{aligned} & \hline $3 . \quad$ Title: $\begin{array}{l}\text { Aplicación del código MC para el estudio de la distibución en 3D de } \\ \text { neutrones en un búnker de radioterapia y validación con medidas ex- } \\ \text { perimentales. }\end{array} \\ &$\hline Conference: $44^{\text {a }}$ Reunión Anual de la Sociedad Nuclear Española \\ & Year: 2018 \\ & Authors: $\begin{array}{l}\text { C. García; Juste Vidal, Belen Jeanine; Morató-Rafet, Sergio; Miró Herrero, } \\ \text { Rafael; Verdú Martín, Gumersindo Jesús }\end{array} \\ &$\hline\end{aligned}

\begin{tabular}{|c|c|}
\hline Title: & $\begin{array}{l}\text { Desarrollo de un código de transporte neutrónico } 1 \mathrm{D} / 2 \mathrm{D} \text { mediante el } \\
\text { método de ordenadas discretas en MATLAB. }\end{array}$ \\
\hline Conference: & dad Nuclear Esp \\
\hline Year: & 2018 \\
\hline Authors: & $\begin{array}{l}\text { Morató-Rafet, Sergio; Bernal-Garcia, Alvaro; Miró Herrero, Rafael; Jose E. } \\
\text { Roman; Verdú Martín, Gumersindo Jesús }\end{array}$ \\
\hline
\end{tabular}

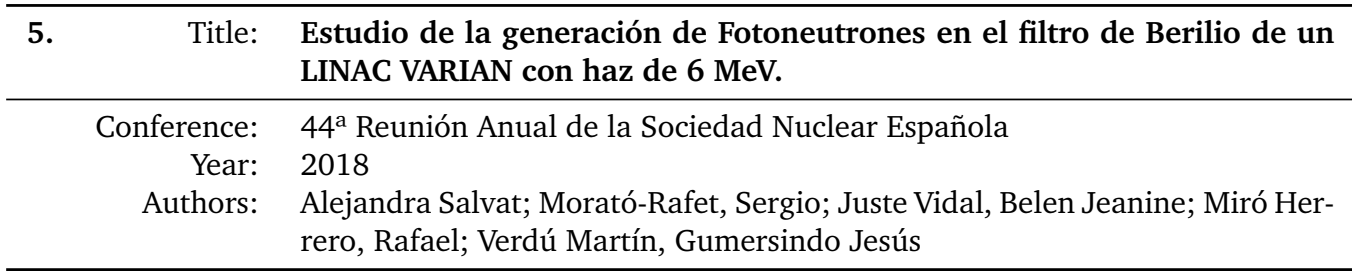

\begin{tabular}{rll}
\hline 6. & Title: & Resultados del código n-DOTEC para el benchmark C5G7. \\
\hline Conference: & $44^{\text {a }}$ Reunión Anual de la Sociedad Nuclear Española \\
Year: & 2018 \\
Authors: & Morató-Rafet, Sergio; Bernal-Garcia, Alvaro; Miró Herrero, Rafael; Jose E. \\
& Roman; Verdú Martín, Gumersindo Jesús \\
\hline
\end{tabular}
7. Title: Aplicación del código MC para el estudio de la distribución en 3D de neutrones en un bunker de radioterapia y validación con medidas ex- perimentales.
Conference: $\quad 43^{\text {a }}$ Reunión Anual de la Sociedad Nuclear Española
Year: 2017
Authors: C. García; Morató-Rafet, Sergio; Juste Vidal, Belen Jeanine; R. Miró; Verdú Martín, Gumersindo Jesús




\begin{tabular}{|c|c|}
\hline Title: & $\begin{array}{l}\text { Cálculo de la estimación de dosis en órganos en braquiterapia mediante } \\
\text { simulaciones Monte Carlo y modelos realistas de pacientes. }\end{array}$ \\
\hline Conference: & $43^{a}$ Reunión Anual de la Sociedad Nuclear Española \\
\hline Year: & 2017 \\
\hline Authors: & $\begin{array}{l}\text { S. Peris; Morató-Rafet, Sergio; Juste Vidal, Belen Jeanine; Miró Herrero, } \\
\text { Rafael }\end{array}$ \\
\hline
\end{tabular}

\begin{tabular}{|c|c|c|}
\hline 9. & Title: & $\begin{array}{l}\text { Cálculo de la Matriz Respuesta de un Espectrómetro de Esferas Bonner } \\
\text { con librerías ENDF/B-VII. }\end{array}$ \\
\hline & Conference: & $43^{\mathrm{a}}$ Reunión Anual de la Sociedad Nuclear Española \\
\hline & Year: & 2017 \\
\hline & Authors: & $\begin{array}{l}\text { Morató-Rafet, Sergio; Juste Vidal, Belen Jeanine; Miró Herrero, Rafael; } \\
\text { Verdú Martín, Gumersindo Jesús }\end{array}$ \\
\hline
\end{tabular}

10. Title: Cálculo de Dosis en Adquisición de Imágenes por Tomografía Computa-

Conference: $\quad 5^{\circ}$ Congreso Conjunto Sociedad Española de Física Médica y Sociedad Española de Protección Radiológica

Year: 2017

Authors: C. García; Morató-Rafet, Sergio; Miró Herrero, Rafael; Juste Vidal, Belen Jeanine; Verdú Martín, Gumersindo Jesús; I. Torres; Candela-Juan, Cristian

\begin{aligned} 11. Title: & $\begin{array}{l}\text { Validación de un Detector de Neutrones mediante Simulación } \\ \text { MCNP6.1.1 en un LinAc Médico Varian TrueBeam. }\end{array} \\$\hline Conference: & $\begin{array}{l}5^{\circ} \text { Congreso Conjunto Sociedad Española de Física Médica y Sociedad Es- } \\ \text { pañola de Protección Radiológica }\end{array} \\$ Year: & 2017 \\ Authors: & $\begin{array}{l}\text { L. Martínez; Juste Vidal, Belen Jeanine; Morató-Rafet, Sergio; Miró Herrero, } \\ \text { Rafael; Verdú Martín, Gumersindo Jesús }\end{array}\end{aligned}$

12. Title: Cálculo de Dosis en Adquisición de Imágenes en Tomografía Computarizada.

Conference: Congreso Anual de la Sociedad Española de Ingeniería Biomédica (CASEIB)

Year: 2016

Authors: C. García; Morató-Rafet, Sergio; M. Lorduy; Miró Herrero, Rafael; Juste Vidal, Belen Jeanine; Verdú Martín, Gumersindo Jesús; I. Torres; C. Candela 


\begin{tabular}{|c|c|c|}
\hline 13. & Title: & $\begin{array}{l}\text { Ventajas del uso de las herramientas de mallado en MCNP6: Tres estu- } \\
\text { dios de casos médicos. }\end{array}$ \\
\hline & $\begin{array}{r}\text { Conference: } \\
\text { Year: }\end{array}$ & $\begin{array}{l}\text { Congreso Anual de la Sociedad Española de Ingeniería Biomédica (CASEIB) } \\
2016\end{array}$ \\
\hline & Authors: & $\begin{array}{l}\text { Morató-Rafet, Sergio; Juste Vidal, Belen Jeanine; Miró Herrero, Rafael; } \\
\text { Verdú Martín, Gumersindo Jesús }\end{array}$ \\
\hline
\end{tabular}

\begin{tabular}{|c|c|}
\hline Title: & $\begin{array}{l}\text { Cálculo de Dosis en Adquisición de Imágenes por Tomografía Computa- } \\
\text { rizada. }\end{array}$ \\
\hline Conference: & de la Sociedad Nuclear Española \\
\hline Year: & 2016 \\
\hline Authors: & $\begin{array}{l}\text { C. García; Morató-Rafet, Sergio; M. Lorduy; Miró Herrero, Rafael; Juste } \\
\text { Vidal, Belen Jeanine; Verdú Martín, Gumersindo Jesús; I.Torres; C. Candela }\end{array}$ \\
\hline
\end{tabular}

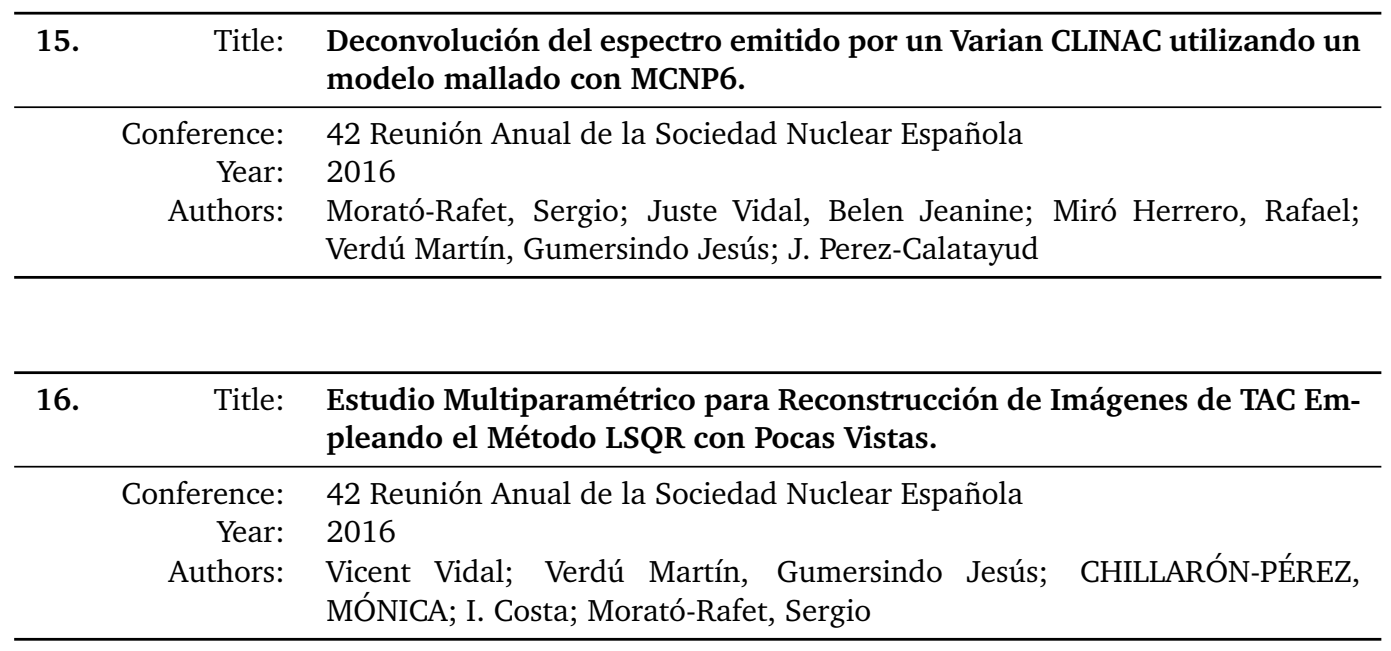

\begin{aligned} & 17. Title: $\begin{array}{l}\text { Validación de la simulación de la activación neutrónica generada en el } \\ \text { Varian CLInac mediante medidas con detector de Germanio. }\end{array} \\ &$\hline Conference: 42 Reunión Anual de la Sociedad Nuclear Española \\ & Year: 2016 \\ & Authors: $\begin{array}{l}\text { Juste Vidal, Belen Jeanine; Morató-Rafet, Sergio; Miró Herrero, Rafael; } \\ \text { Verdú Martín, Gumersindo Jesús; Sergio Díez Domingo }\end{array} \\ &$\hline\end{aligned}




\begin{tabular}{|c|c|c|}
\hline 18. & Title: & $\begin{array}{l}\text { Distribución neutrónica y comprobación de blindajes en el interior de } \\
\text { una sala de tratamiento radioterapéutico mediante geometría de malla } \\
\text { no estructurada. }\end{array}$ \\
\hline & Conference: & 41 Reunión Anual de la Sociedad Nuclear Española \\
\hline & Year: & 2015 \\
\hline & Authors: & Morató-Rafet, Sergio; Juste Vidal, Belen Jeanine; Miró Herrero, Rafael; \\
\hline & & Verdú Martín, Gumersindo Jesús; Sergio Diez Domingo \\
\hline \multirow[t]{4}{*}{19.} & Title: & $\begin{array}{l}\text { Estudio de la dosis por fotoneutrones en tratamientos de radioterapia } \\
\text { por medio de simulación MCNP6 y malla no estructurada. }\end{array}$ \\
\hline & Conference: & 41 Reunión Anual de la Sociedad Nuclear Española \\
\hline & Year: & 2015 \\
\hline & Authors: & $\begin{array}{l}\text { Morató-Rafet, Sergio; Juste Vidal, Belen Jeanine; Miró Herrero, Rafael; } \\
\text { Verdú Martín. Gumersindo Jesús: Agustín Santos Serra }\end{array}$ \\
\hline & & \\
\hline \multirow[t]{4}{*}{20.} & Title: & $\begin{array}{l}\text { Planificación de radioterapia mediante el uso de modelos realistas per- } \\
\text { sonalizados de pacientes a partir de imagen TC con malla no estruc- } \\
\text { turada. }\end{array}$ \\
\hline & Conference: & 41 Reunión Anual de la Sociedad Nuclear Española \\
\hline & Year: & 2015 \\
\hline & Authors: & $\begin{array}{l}\text { Morató-Rafet, Sergio; Miró Herrero, Rafael; Juste Vidal, Belen Jeanine; } \\
\text { Verdú Martín, Gumersindo Jesús }\end{array}$ \\
\hline \multirow[t]{4}{*}{21.} & Title: & $\begin{array}{l}\text { Planificación de Radioterapia mediante el uso de modelos realistas per- } \\
\text { sonalizados de pacientes a partir de imágenes TC con malla no estruc- } \\
\text { turada. }\end{array}$ \\
\hline & Conference: & $\begin{array}{l}4^{\circ} \text { Congreso Conjunto Sociedad Española de Física Médica y Sociedad Es- } \\
\text { pañola de Protección Radiológica }\end{array}$ \\
\hline & Year: & 2015 \\
\hline & Authors: & $\begin{array}{l}\text { Morató-Rafet, Sergio; Juste Vidal, Belen Jeanine; Miró Herrero, Rafael; } \\
\text { Verdú Martín, Gumersindo Jesús }\end{array}$ \\
\hline
\end{tabular}

22.

Title: Reconstrucción de imagen y estimación de dosis en órgano en Tomografía Computarizada utilizando simulaciones Monte Carlo.

Conference: $\quad 4^{\circ}$ Congreso Conjunto Sociedad Española de Física Médica y Sociedad Española de Protección Radiológica

Year: 2015

Authors: Miró Herrero, Rafael; Morató-Rafet, Sergio; Juste Vidal, Belen Jeanine; María; Verdú Martín, Gumersindo Jesús; I. Torres; E. Ruiz; J. A. Bautista; C. Candela; PEREZ, J.; B. Ibáñez-Roselló 


\subsection{Future works}

Three different approximations of the Neutron Transport Equation were studied in the work developed in this thesis. Then, a lot of possibilities appear opening new avenues for research. Next the author listed some of them:

- Because the $S_{N}$ calculation time increases with the spatial or angular discretization and with the number of groups, for practical purposes in the future it is expected to implement the algorithms in parallel to accelerate the calculations.

- Future works could develop the solution of $S_{N}$ and $S P_{3}$ for non-multiplying systems with other kind of particles (photons) and the solution of problems with fixed source, which are interesting in shielding and radiation protection.

- Programming of the transient version of all the codes developed in order to simulate more realistic reactor core problems.

- Pin and lattice homogenization studies could be very interesting, as well as investigate about a dynamic homogenization iterative methodology that involves $S_{N}$ and $S P_{3}$.

- Development of new finite difference techniques and research about the convergence of these methods.

- Implementation of higher order Simplified Spherical Harmonics approximations like $S P_{5}$ or $S P_{7}$.

- Implementation of other different discretization methods, such as Finite Elemente Method or Finite Volume Method.

- Compare the results presented in the thesis with other approximation to the Neutron Transport Equations, for example Method of Characteristics.

- The development of a 2D/1D method which could be used to solve transport problems using $S P_{3}$ method for the axial direction and $S_{N}$ method for the radial. 



\section{Bibliography}

M. L. Adams and E. W. Larsen. Fast iterative methods for discrete-ordinates particle transport calculations. Progress in nuclear energy, 40(1):3-159, 2002. (page 12)

R. E. Alcouffe. Threedant: a code to perform three-dimensional, neutral particle transport calculations. Technical report, Los Alamos National Lab., 1994. (page 12)

R. E. Alcouffe, R. S. Baker, F. W. Brinkley, D. R. Marr, R. D. O'Dell, and W. F. Walters. Dantsys: a diffusion accelerated neutral particle transport code system. 1995. (page 10)

R. E. Alcouffe, R. S. Baker, J. A. Dahl, S. A. Turner, and R. Ward. Partisn: A timedependent, parallel neutral particle transport code system. Los Alamos National Laboratory, LA-UR-05-3925 (May 2005), 2005. (page 10, 12)

P. R. Amestoy, I. S. Duff, and J.-Y. L'Excellent. Mumps multifrontal massively parallel solver version 2.0. 1998. (page 21, 97)

J. Askew. A characteristics formulation of the neutron transport equation in complicated geometries. Technical report, United Kingdom Atomic Energy Authority, 1972. (page 10)

Y. Azmy and E. Sartori. Nuclear computational science: a century in review. Springer, 2010. (page 6, 11, 49)

Y. Y. Azmy. The weighted diamond-difference form of nodal transport methods. Nuclear Science and Engineering, 98(1):29-40, 1988. (page 17) 
T. Bahadir, S.-Ö. Lindahl, and S. P. Palmtag. Simulate-4 multigroup nodal code with microscopic depletion model. Mathematics and Computation, Supercomputing, Reactor Physics and Nuclear and Biological Applications, 2005. (page 11)

S. Balay, S. Abhyankar, M. F. Adams, J. Brown, P. Brune, K. Buschelman, L. Dalcin, A. Dener, V. Eijkhout, W. D. Gropp, D. Karpeyev, D. Kaushik, M. G. Knepley, D. A. May, L. C. McInnes, R. T. Mills, T. Munson, K. Rupp, P. Sanan, B. F. Smith, S. Zampini, H. Zhang, and H. Zhang. PETSc users manual. Technical Report ANL-95/11 - Revision 3.11, Argonne National Laboratory, 2019. URL http://www.mcs.anl.gov/petsc. (page 21, 71, 127)

C. Beckert and U. Grundmann. Development and verification of a nodal approach for solving the multigroup sp3 equations. Annals of Nuclear Energy, 35(1):7586, 2008. (page 15)

G. Bell and S. Glasstone. Nuclear reactor theory, krieger, 1985. (page 54)

G. I. Bell and S. Glasstone. Nuclear reactor theory. Technical report, US Atomic Energy Commission, Washington, DC (United States), 1970. (page 6)

J. Bennett. Txy01-a one-group transport program for xy geometry. Technical report, Westinghouse Electric Corp. Bettis Atomic Power Lab., Pittsburgh, 1964. (page 16)

A. Bernal, S. Morató, R. Miró, and G. Verdú. A gauss-legendre product quadrature for the neutron transport equation. (page 60,68)

Á. Bernal, A. Hébert, J. E. Roman, R. Miró, and G. Verdú. A krylov-schur solution of the eigenvalue problem for the neutron diffusion equation discretized with the raviart-thomas method. Journal of Nuclear Science and Technology, 54(10): 1085-1094, 2017. (page 19, 71)

A. Bernal, R. Miro, G. Verdu, and J. Roman. Valkin-fvm: A modal finite volume method for solving the transient neutron diffusion equation on unstructured meshes. 2018a. (page 11)

A. Bernal, J. E. Roman, R. Miró, and G. Verdú. Calculation of multiple eigenvalues of the neutron diffusion equation discretized with a parallelized finite volume method. Progress in Nuclear Energy, 105:271-278, 2018b. (page 19)

Á. Bernal García. Development of a 3D Modal Neutron Code with the Finite Volume Method for the Diffusion and Discrete Ordinates Transport Equations. Application to Nuclear Safety Analyses. PhD thesis, 2018. (page 18, 54, 218) 
R. N. Blomquist. Buckled spherical harmonics solutions of neutron transport problems. 1979. (page 10)

P. S. Brantley and E. W. Larsen. The simplified p 3 approximation. Nuclear Science and Engineering, 134(1):1-21, 2000. (page 15, 80, 164, 165, 172)

D. G. Cacuci. Handbook of Nuclear Engineering: Vol. 1: Nuclear Engineering Fundamentals; Vol. 2: Reactor Design; Vol. 3: Reactor Analysis; Vol. 4: Reactors of Generations III and IV; Vol. 5: Fuel Cycles, Decommissioning, Waste Disposal and Safeguards, volume 2. Springer Science \& Business Media, 2010. (page 6, 14, $25,30,32,108)$

L.-Z. Cao and H.-C. Wu. Spherical harmonics method for neutron transport equation based on unstructured-meshes. Nucl. Sci. Tech, 15(6):335-339, 2004. (page 10)

M. Capilla, C. Talavera, D. Ginestar, and G. Verdú. A nodal collocation method for the calculation of the lambda modes of the pl equations. Annals of Nuclear Energy, 32(17):1825-1853, 2005. (page 136, 137, 141, 191, 198)

M. Capilla, C. Talavera, D. Ginestar, and G. Verdú. A nodal collocation approximation for the multi-dimensional pl equations-2d applications. Annals of Nuclear Energy, 35(10):1820-1830, 2008. (page 10, 75, 80, 81, 136, 191, 202)

M. Capilla, C. Talavera, D. Ginestar, and G. Verdú. Application of a nodal collocation approximation for the multidimensional pl equations to the $3 \mathrm{~d}$ takeda benchmark problems. Annals of Nuclear Energy, 40(1):1-13, 2012. (page 191, 222)

M. Capilla, C. Talavera, D. Ginestar, and G. Verdú. Nodal collocation method for the multidimensional pl equations applied to neutron transport source problems. Annals of Nuclear Energy, 87:89-100, 2016. (page 97, 100, 101)

M. Capilla, C. Talavera, D. Ginestar, and G. Verdú. Numerical analysis of the 2d c5g7 mox benchmark using pl equations and a nodal collocation method. Annals of Nuclear Energy, 114:32-41, 2018. (page xxviii, xxix, 89, 154, 158)

J. F. Carew and G. Zamonsky. Uniform positive-weight quadratures for discrete ordinate transport calculations. Nuclear science and engineering, 131(2):199207, 1999. (page 14)

B. G. Carlson. Transport theory-the method of discrete ordinates. Computing methods in reactor physics, 1968. (page 6, 11, 16) 
B. G. Carlson. Transport theory: Discrete ordinates quadrature over the unit sphere. Technical report, Los Alamos Scientific Lab., N. Mex., 1970. (page 13, 60)

B. G. Carlson. Tables of equal weight quadrature eqn over the unit sphere. Los Alamos Scientific Laboratory of the University of California, 1971. (page 60)

A. Carreño, A. Vidal-Ferràndiz, D. Ginestar, and G. Verdú. Spatial modes for the neutron diffusion equation and their computation. Annals of Nuclear Energy, 110:1010-1022, 2017. (page 19)

Y.-A. Chao. A new and rigorous spn theory for piecewise homogeneous regions. Annals of Nuclear Energy, 96:112-125, 2016. (page 15)

Y. Chen, B. Zhang, L. Zhang, J. Zheng, Y. Zheng, and C. Liu. Ares: a parallel discrete ordinates transport code for radiation shielding applications and reactor physics analysis. Science and Technology of Nuclear Installations, 2017, 2017. (page 10, 12)

B. Collins, B. Kochunas, and T. Downar. Assessment of the $2 \mathrm{~d}$ moc solver in mpact: Michigan parallel characteristics transport code. In Proceedings of the 2013 International Conference on Mathematics and Computational Methods Applied to Nuclear Science and Engineering-M and C 2013, 2013. (page 10)

B. Collins, S. Stimpson, B. W. Kelley, M. T. Young, B. Kochunas, A. Graham, E. W. Larsen, T. Downar, and A. Godfrey. Stability and accuracy of 3d neutron transport simulations using the $2 \mathrm{~d} / 1 \mathrm{~d}$ method in mpact. Journal of Computational Physics, 326:612-628, 2016. (page 11)

J. Davis, L. Hageman, and R. Kellogg. Singular difference approximations for the discrete ordinate equations in xy geometry. Nuclear Science and Engineering, 29 (2):237-243, 1967. (page 16)

B. Davison, J. B. Sykes, and E. R. Cohen. Neutron transport theory. PhT, 11(2): 30, 1958. (page 6)

K. Deo, P. Krishnani, and R. Modak. Development of one-dimensional neutron transport theory code based on method of characteristics. Technical report, Bhabha Atomic Research Centre, 2014. (page 75, 136)

T. Downar, D. Lee, Y. Xu, T. Kozlowski, and J. Staudenmier. Parcs v2. 6 us nrc core neutronics simulator theory manual. School of Nuclear Engineering Purdue University, 2004. (page 11)

J. J. Duderstadt and L. J. Hamilton. Nuclear reactor analysis, johnwiley \& sons. Inc., New York, 1976a. (page 6) 
J. J. Duderstadt and L. J. Hamilton. Nuclear reactor analysis. 1976. Ann Arbor, Michigan: Wiley-Interscience, 650, 1976b. (page 27)

J. J. Duderstadt and W. R. Martin. Transport theory. trth, 1979. (page 6)

J. Duo and Y. Azmy. Error comparison of diamond difference, nodal, and characteristic methods for solving multidimensional transport problems with the discrete ordinates approximation. Nuclear science and engineering, 156(2):139153, 2007. (page 17)

T. M. Evans, A. S. Stafford, R. N. Slaybaugh, and K. T. Clarno. Denovo: A new three-dimensional parallel discrete ordinates code in scale. Nuclear technology, 171(2):171-200, 2010. (page 10, 12, 17)

N. García-Herranz, D. Cuervo, A. Sabater, G. Rucabado, S. Sánchez-Cervera, and E. Castro. Multiscale neutronics/thermal-hydraulics coupling with cobaya4 code for pin-by-pin pwr transient analysis. Nuclear Engineering and Design, 321:38-47, 2017. (page 11)

E. M. Gelbard. Application of spherical harmonics method to reactor problems. Bettis Atomic Power Laboratory, West Mifflin, PA, Technical Report No. WAPD-BT20, 1960. (page 15, 164)

E. M. Gelbard. Simplified spherical harmonics equations and their use in shielding problems. Technical report, Westinghouse Electric Corp. Bettis Atomic Power Lab., Pittsburgh, 1961. (page 15)

I. Gueye, S. E. Arem, F. Feyel, F.-X. Roux, and G. Cailletaud. A new parallel sparse direct solver: Presentation and numerical experiments in large-scale structural mechanics parallel computing. International Journal for Numerical Methods in Engineering, 88(4):370-384, 2011. (page 71)

G. Habetler and B. Matkowsky. Uniform asymptotic expansions in transport theory with small mean free paths, and the diffusion approximation. Journal of Mathematical Physics, 16(4):846-854, 1975. (page 104)

M. Halsall. Cactus, a characteristics solution to the neutron transport equations in complicated geometries. Technical report, UKAEA Atomic Energy Establishment, 1980. (page 10)

S. P. Hamilton and T. M. Evans. Efficient solution of the simplified pn equations. Journal of Computational Physics, 284:155-170, 2015. (page 15, 165, 172)

A. Hébert. Applied reactor physics. Presses inter Polytechnique, 2009. (page 6, $62,116)$ 
A. Hébert. Mixed-dual implementations of the simplified pn method. Annals of Nuclear Energy, 37(4):498-511, 2010. (page 15)

A. F. Henry et al. Nuclear-reactor analysis, volume 4. MIT press Cambridge, Massachusetts, 1975. (page 6)

V. Hernandez, J. E. Roman, and V. Vidal. Slepc: A scalable and flexible toolkit for the solution of eigenvalue problems. ACM Transactions on Mathematical Software (TOMS), 31(3):351-362, 2005. (page 20, 71, 127)

V. Hernández, J. E. Román, A. Tomás, and V. Vidal. Krylov-schur methods in slepc. Universitat Politecnica de Valencia, Tech. Rep. STR-7, 2007. (page 19)

J. Issa, N. Riyait, A. Goddard, and G. Stott. Multigroup application of the anisotropic fem code feltran to one, two, three-dimensions and rz problems. Progress in Nuclear Energy, 18(1-2):251-264, 1986. (page 75, 76, 136)

H.-T. Ju, H.-C. Wu, Y.-Q. Zhou, L.-Z. Cao, D. Yao, and C.-Y. Xian. A least-squares finite-element sn method for solving first-order neutron transport equation. $\mathrm{Nu}$ clear Engineering and Design, 237(8):823-829, 2007. (page 100)

Y. Jung. ntracer v1. 0 methodology manual. SNURPL-CMO01 (10), Seoul National University Reactor Physics Laboratory, Seoul, Republic of Korea, 2010. (page 10)

B. Juste, S. Morató, R. Miró, G. Verdú, and S. Díez. Mcnp6 unstructured mesh application to estimate the photoneutron distribution and induced activity inside a linac bunker. Radiation Physics and Chemistry, 137:18-22, 2017. (page 228)

B. Juste, S. Morató, C. García, R. Miró, and G. Verdú. Monte carlo code application to the study of $3 \mathrm{~d}$ neutrons distribution in a radiotherapy bunker and validation with experimental measurements. Nuclear Instruments and Methods in Physics Research Section A: Accelerators, Spectrometers, Detectors and Associated Equipment, 2018a. (page 228)

B. Juste, S. Morató, A. Salvat, R. Miró, and G. Verdú. Study of the photoneutron generation caused by a linac beryllium window with a 6 mev treatment beam. In 2018 40th Annual International Conference of the IEEE Engineering in Medicine and Biology Society (EMBC), pages 4150-4153. IEEE, 2018b. (page 228)

B. Juste, R. Miró, S. Morató, G. Verdú, and S. Peris. Prostate cancer monte carlo dose model with 177lutetium and 125iodine treatments. Radiation Physics and Chemistry, page 108908, 2020a. (page 96) 
B. Juste, S. Morató, R. Miró, A. I. Prieto, G. Verdú, R. Genoves, and J. Gimeno. Development of a reconstruction methodology for the x-ray spectrum of a medical linac positioning flat panel. Radiation Physics and Chemistry, 167:108307, 2020b. (page 96)

S. Kashi, A. Minuchehr, A. Zolfaghari, and B. Rokrok. Mesh-free method for numerical solution of the multi-group discrete ordinate neutron transport equation. Annals of Nuclear Energy, 106:51-63, 2017. (page 75, 83, 86, 136)

K. Kobayashi. A proposal for 3-d radiation transport benchmarks for simple geometries with void region. In 3-D deterministic radiation transport computer programs. Features, applications and perspectives, pages 403-410. Nuclear Energy Agency of the OECD (NEA), 1997. (page 101)

R. Koch and R. Becker. Evaluation of quadrature schemes for the discrete ordinates method. Journal of Quantitative Spectroscopy and Radiative Transfer, 84 (4):423-435, 2004. (page 13)

R. Koch, W. Krebs, S. Wittig, and R. Viskanta. Discrete ordinates quadrature schemes for multidimensional radiative transfer. Journal of Quantitative Spectroscopy and Radiative Transfer, 53(4):353-372, 1995. (page 13)

D. E. Kornreich and D. K. Parsons. The green's function method for effective multiplication benchmark calculations in multi-region slab geometry. Annals of Nuclear Energy, 31(13):1477-1494, 2004. (page 75, 77, 136)

P. Kotiluoto, J. Pyyry, and H. Helminen. Multitrans sp3 code in coupled photonelectron transport problems. Radiation Physics and Chemistry, 76(1):9-14, 2007. (page 15)

J. R. Lamarsh and A. J. Baratta. Introduction to nuclear engineering, volume 3. Prentice hall Upper Saddle River, NJ, 2001. (page 14)

E. W. Larsen. Hybrid monte carlo-deterministic methods for neutral particle transport problems. (page 9)

E. W. Larsen. Diffusion theory as an asymptotic limit of transport theory for nearly critical systems with small mean free paths. Annals of Nuclear Energy, 7(4-5): 249-255, 1980. (page 104)

E. W. Larsen. An overview of neutron transport problems and simulation techniques. pages 513-534, 2006. (page 6, 8)

E. W. Larsen and R. Hughes. Homogenized diffusion approximations to the neutron transport equation. Nuclear Science and Engineering, 73(3):274-285, 1980. (page 104) 
E. W. Larsen, J. E. Morel, and J. M. McGhee. Asymptotic derivation of the multigroup p 1 and simplified pn equations with anisotropic scattering. Nuclear science and engineering, 123(3):328-342, 1996. (page 15, 104, 172)

K. Lathrop. Threetran-a program to solve the multigroup discrete ordinates transport equation in (x, y, z) geometry. Technical report, SIS-76-4011, 1976. (page 11)

K. D. Lathrop. Dtf-iv : a fortran-iv program for solving the multigroup transport equation with anisotropic scattering. 1965. (page 11)

K. D. Lathrop. Ray effects in discrete ordinates equations. Nuclear Science and Engineering, 32(3):357-369, 1968. (page 11)

K. D. Lathrop and B. G. Carlson. Discrete ordinates angular quadrature of the neutron transport equation. Technical report, Los Alamos Scientific Lab., N. Mex., 1964. (page 11, 12, 13, 42, 60, 164)

D. Lee, T. Kozlowski, and T. J. Downar. Multi-group sp3 approximation for simulation of a three-dimensional pwr rod ejection accident. Annals of Nuclear Energy, 77:94-100, 2015. (page 11, 13)

E. E. Lewis. Fundamentals of nuclear reactor physics. Elsevier, 2008. (page 6)

E. E. Lewis and W. F. Miller Jr. Computational methods of neutron transport. 1984, 1984. (page 6, 11, 25, 30, 32, 33, 46, 48, 51, 54)

F. Liu, H. Becker, and A. Pollard. Spatial differencing schemes of the discreteordinates method. Numerical Heat Transfer, 30(1):23-43, 1996. (page 17)

N. K. Madsen. Weighted central difference equations for the neutron transport equation. Nuclear Science and Engineering, 54(4):376-386, 1974. (page 16)

G. Marchuk and V. I. Lebedev. Numerical methods in the theory of neutron transport. 1986. (page 11)

G. Marleau, A. Hébert, and R. Roy. A user guide for dragon 3.06. Report IGE-174 Rev, 7, 2008. (page 10, 12)

R. G. McClarren. Theoretical aspects of the simplified pn equations. Transport Theory and Statistical Physics, 39(2-4):73-109, 2010. (page 15)

S. Morató, B. Juste, R. Miró, and G. Verdú. Dose calculation from photoneutrons emitted in radiotherapy treatments by means of mcnp6 simulation and unstructured mesh. In 2015 IEEE Nuclear Science Symposium and Medical Imaging Conference (NSS/MIC), pages 1-4. IEEE, 2015. (page 228) 
S. Morató, B. Juste, R. Miró, G. Verdú, and S. Diez. Experimental validation of neutron activation simulation of a varian medical linear accelerator. In 2016 38th Annual International Conference of the IEEE Engineering in Medicine and Biology Society (EMBC), pages 5656-5659. IEEE, 2016. (page 228)

S. Morató, C. Garcia, B. Juste, R. Miró, and G. Verdú. Dose calculation in computerized tomography. In 2017 39th Annual International Conference of the IEEE Engineering in Medicine and Biology Society (EMBC), pages 560-563. IEEE, 2017. (page 96)

S. Morató, B. Juste, S. Peris, R. Miró, G. Verdú, F. Ballester, and J. Vijande. Brachytherapy organ dose estimation using monte carlo simulations of realistic patient models. In 2018 40th Annual International Conference of the IEEE Engineering in Medicine and Biology Society (EMBC), pages 6149-6152. IEEE, 2018. (page 96)

S. Morató, B. Juste, R. Miró, G. Verdú, and V. Guardia. Evaluation of the response of a bonner sphere spectrometer with a 6lii detector using 3d meshed monp6. 1.1 models. Radiation Physics and Chemistry, 155:221-224, 2019. (page 96)

S. Morató, Á. Bernal, R. Miró, J. E. Roman, and G. Verdú. Calculation of $\lambda$ modes of the multi-group neutron transport equation using the discrete ordinates and finite difference method. Annals of Nuclear Energy, 137:107077, 2020. (page 136, 212)

M. Natelson. Variational derivation of discrete ordinate-like approximations. $\mathrm{Nu}$ clear Science and Engineering, 43(2):131-144, 1971. (page 16)

R. K. Osborn and S. Yip. The foundations of neutron transport theory. fntt, 1966. (page 6)

G. C. Papanicolaou. Asymptotic analysis of transport processes. Bulletin of the American Mathematical Society, 81(2):330-392, 1975. (page 104)

R. Piessens and E. De Doncker-Kapenga. U è berhuber c and kahaner d (1983) quadpack, a subroutine package for automatic integration. Springer Ser. Comput. Math, 1. (page 97)

G. C. Pomraning. Asymptotic and variational derivations of the simplified pn equations. Annals of nuclear energy, 20(9):623-637, 1993. (page 15)

W. Rhoades. Dot iv variable mesh discrete ordinates transport code. Technical report, Oak Ridge National Lab., TN (USA), 1977. (page 17) 
W. Rhoades and R. Childs. Dort/tort two-and three-dimensional discrete ordinates transport, version 2.7. 3. ornl, oak ridge. Technical report, RSIC-CCC543, 1993. (page 10)

W. Rhoades and W. Engle Jr. New weighted-difference formulation for discreteordinates calculations. Technical report, Oak Ridge National Lab., Tenn.(USA), 1977. (page 16)

W. A. Rhoades and D. Simpson. The tort three-dimensional discrete ordinates neutron/photon transport code (tort version 3). Technical report, Oak Ridge National Lab., TN (United States), 1997. (page 12, 17)

S. Rukolaine and V. Yuferev. Discrete ordinates quadrature schemes based on the angular interpolation of radiation intensity. Journal of Quantitative Spectroscopy and Radiative Transfer, 69(3):257-275, 2001. (page 13)

Y. Saad and M. H. Schultz. Gmres: A generalized minimal residual algorithm for solving nonsymmetric linear systems. SIAM Journal on scientific and statistical computing, 7(3):856-869, 1986. (page 18, 127)

R. Sanchez and N. J. McCormick. A review of neutron transport approximations. Nuclear Science and Engineering, 80(4):481-535, 1982. (page 11)

G. Sjoden and A. Haghighat. Pentran?: Parallel environment neutral particle transport in 3-d cartesian geometry. In Proc. Int. Conf. on Mathematical Methods and Supercomputing for Nuclear Applications, pages 232-234, 1997. (page 10)

G. L. Sleijpen and H. A. Van der Vorst. An overview of approaches for the stable computation of hybrid bicg methods. Applied numerical mathematics, 19(3): 235-254, 1995. (page 127)

M. Smith, E. Lewis, and B. Na. Benchmark on deterministic transport calculations without spatial homogenization: A 2-d/3-d mox fuel assembly 3-d benchmark, 2003. (page $75,88,136,158$ )

A. Sood, R. A. Forster, and D. K. Parsons. Analytical benchmark test set for criticality code verification. Progress in Nuclear Energy, 42(1):55-106, 2003. (page xxvii, 75, 78, 79)

W. M. Stacey. Nuclear reactor physics, volume 2. Wiley Online Library, 2007. (page 6, 104)

J. Stepanek, T. Auerbach, and W. Hälg. Calculation of four thermal reactor benchmark problems in xy geometry. Technical report, Eidgenoessisches Inst. fuer Reaktorforschung, 1982. (page 75, 83, 136) 
G. W. Stewart. A krylov-schur algorithm for large eigenproblems. SIAM Journal on Matrix Analysis and Applications, 23(3):601-614, 2002. (page 19)

K. Tada, A. Yamamoto, Y. Yamane, and Y. Kitamuray. Applicability of the diffusion and simplified p3 theories for pin-by-pin geometry of bwr. Journal of nuclear science and technology, 45(10):997-1008, 2008. (page 165)

T. Takeda and H. Ikeda. 3-d neutron transport benchmarks. Journal of Nuclear Science and Technology, 28(7):656-669, 1991. (page 191, 222)

K. Talaat. Development of a one dimensional sn method neutron transport solver. (page 75,136$)$

C. Thurgood, A. Pollard, and H. Becker. The tn quadrature set for the discrete ordinates method. 1995. (page 13, 14)

D. I. Tomašević and E. W. Larsen. The simplified p 2 approximation. Nuclear science and engineering, 122(3):309-325, 1996. (page 15)

P. J. Turinsky, R. M. Al-Chalabi, P. Engrand, H. N. Sarsour, F. X. Faure, and W. Guo. Nestle: Few-group neutron diffusion equation solver utilizing the nodal expansion method for eigenvalue, adjoint, fixed-source steady-state and transient problems. Technical report, EG and G Idaho, Inc., Idaho Falls, ID (United States); Los Alamos National ?, 1994. (page 11)

G. Verdú, R. Miró, D. Ginestar, and V. Vidal. Transients modal analysis using trac-bf1/valkin. PHYSOR 2002, 2002. (page 11)

A. Vidal Ferràndiz. Development of a finite element method for neutron transport equation approximations. PhD thesis, 2018. (page 191, 192)

A. Vidal-Ferrandiz, R. Fayez, D. Ginestar, and G. Verdú. Solution of the lambda modes problem of a nuclear power reactor using an h-p finite element method. Annals of Nuclear Energy, 72:338-349, 2014. (page 19)

A. Vidal-Ferràndiz, S. González-Pintor, D. Ginestar, A. Carreño, and G. Verdú. Optimized eigenvalue solvers for the neutron transport equation. In International Conference on Computational Science, pages 823-832. Springer, 2018a. (page $71,136)$

A. Vidal-Ferràndiz, S. González-Pintor, D. Ginestar, C. Demazière, and G. Verdú. Pin-wise homogenization for spn neutron transport approximation using the finite element method. Journal of Computational and Applied Mathematics, 330: 806-821, 2018b. (page 11) 
A. Vidal-Ferràndiz, A. Carreño, D. Ginestar, and G. Verdú. A block arnoldi method for the spn equations. International Journal of Computer Mathematics, 0(0): 1-17, 2019. doi: 10.1080/00207160.2019.1602768. URL https://doi .org/ 10.1080/00207160.2019.1602768. (page xxix, 162, 212, 216)

A. Vidal-Ferràndiz, A. Carreño, D. Ginestar, and G. Verdú. A block arnoldi method for the spn equations. International Journal of Computer Mathematics, 97(1-2): 341-357, 2020. (page 15)

S. D. Wai and D. Gottlieb. The chebyshev-legendre method: Implementing legendre methods on chebyshev points. Technical report, INSTITUTE FOR COMPUTER APPLICATIONS IN SCIENCE AND ENGINEERING HAMPTON VA, 1993. (page 60)

W. F. Walters. Use of the chebyshev-legendre quadrature set in discrete-ordinate codes. Technical report, Los Alamos National Lab., NM (USA), 1987. (page 60)

Z. Wu, Q. Zhang, and H. Abdel-Khalik. Hybrid monte carlo-deterministic methods for reactor analysis. Nuclear Technology, 180(3):372-382, 2012. doi: 10.13182/NT12-A15350. URL https://doi.org/10.13182/NT12-A15350. (page 9)

C. Yi. Titan: A 3-d deterministic radiation transport code, titan user manual version 1.05. Univ. of Florida, 2009. (page 10, 12)

M. Youssef, R. Feder, P. Batistoni, U. Fischer, S. Jakhar, C. Konno, M. Loughlin, R. Villari, and Y. Wu. Benchmarking of the 3-d cad-based discrete ordinates code ?attila? for dose rate calculations against experiments and monte carlo calculations. Fusion Engineering and Design, 88(11):3033-3040, 2013. (page $10,12)$

S. Zhuang, P. He, G. Sun, L. Hao, and Y. Wu. Hybrid monte carlo-deterministic method for 3d neutron transport simulation based on energy region division. Annals of Nuclear Energy, 130:271-276, 2019. (page 9)

A. Ziver, M. Shahdatullah, M. Eaton, C. de Oliveira, A. Umpleby, C. Pain, and A. Goddard. Finite element spherical harmonics (pn) solutions of the threedimensional takeda benchmark problems. Annals of Nuclear Energy, 32(9): 925-948, 2005. (page 10)

B. Zolotar and F. Rahn. Bwr rod bundle benchmark problem. Transactions of the American Nuclear Society, 23, 1976. (page 86) 

The most accurate way to know the movement of the neutrons through matter is achieved by solving the Neutron Transport Equation. Three different approaches to solve this equation have been investigated in this thesis: Discrete Ordinates Neutron Transport Equation, Neutron Diffusion Equation and Simplified Spherical Harmonics Equations.

In order to solve the equations, different schemes of the Finite Differences Method were studied. The solution of these equations describe the population of neutrons and the occurred reactions inside a nuclear system. These variables are related with the flux and power, fundamental parameters for the Nuclear Safety Analysis.

The thesis introduces the definition of the mentioned equations. In particular, they are detailed for the steady state case. The Modal Method is proposed as a solution to the eigenvalue problems determined by the equations.

First, several algorithms for the solution of the steady state of the Neutron Transport Equation with the Discrete Ordinates Method for the angular discretization and the Finite Difference Method for spatial discretization are developed. A formulation able to solve eigenvalue problems for any number of energy groups, with scattering and anisotropy has been developed. Several quadratures used by this method for the angular discretization have been studied and implemented for any order of approach of the discrete ordinates. Furthermore, an adapted formulation has been developed as a solution of the source problem for the Neutron Transport Equation.

Next, an algorithm is carried out that allows to solve the Neutron Diffusion Equation with two variants of the Finite Difference Method, one with cell centered scheme and another edge centered. The Modal method is also used for calculating any number of eigenvalues for several energy groups and upscattering.

Both Finite Difference schemes mentioned before are also implemented to solve the Simplified Spherical Harmonics Equations. Moreover, an analysis of different approaches of the boundary conditions is performed.

Finally, calculations of the multiplication factor, subcritical modes, neutron flux and the power for different nuclear reactors were carried out. These variables result essential in Nuclear Safety Analysis. In addition, several sensitivity studies of parameters like mesh size, quadrature order or quadrature type were performed. 